\title{
Nutzung und Effektivität \\ der kategorialen Organisationsstrategie im Grundschulalter
}

\author{
Dissertation \\ zur Erlangung des Doktorgrades \\ der Mathematisch-Naturwissenschaftlichen Fakultäten \\ der Georg-August-Universität zu Göttingen
}

vorgelegt von Maren Richter

aus Bad Pyrmont

Göttingen 2004 
D 7

Referent: Prof. Dr. Marcus Hasselhorn

Korreferent: Prof. Dr. Gerd Lüer

Tag der mündlichen Prüfung: 04.11.2004 


\section{$\underline{\text { Inhaltsverzeichnis }}$}

Vorwort

1. Einleitung

2. Theoretischer und empirischer Hintergrund

2.1 Annahmen zur effektiven Strategieentwicklung 3

2.1.1 Strategisches Verhalten 3

2.1.2 Entwicklung kategorialen Organisationsverhaltens 7

2.1.3 Entwicklung einer effektiven kategorialen Organisationsstrategie 11

2.2 Empirische Überprüfung kategorialer Organisationsprozesse 18

2.2.1 Typische Aufgaben zur Überprüfung des kategorialen Organisierens 19

2.2.2 Externe Faktoren und ihre Bedeutung für die Strategieanwendung 21

2.2.3 Experimentelle Designs 27

2.2.4 Anzeichen des Strategiegebrauchs 39

2.3 Erklärungsannahmen zu Ursachen effektiver Strategieentwicklung 43

2.3.1 Annahmen der Mediationstheorie 43

2.3.2 Automatische Wissensaktivierung (Bjorklund) 44

2.3.3 Strategische Wissensaktivierung (Hasselhorn) 46

2.3.4 Kritische Reflektion der Erklärungsmodelle 49

2.4 Determinanten für die Produktion und Effektivität von Strategien 52

2.4.1 Arbeitsgedächtniskapazität 55

$\begin{array}{ll}2.4 .2 \text { Vorwissen } & 60\end{array}$

2.4.3 Metagedächtnis $\quad 64$

2.4.4 Gemeinsamer Einfluss von kognitiven und nicht-kognitiven Determinanten 77

2.4.5 Gemeinsamer Einfluss von internen und externen Determinanten 84

2.5 Zusammenfassung 86

3. Eigene Fragestellung $\quad 88$

3.1 Die aktuelle empirische Datenlage $\quad 88$

3.2 Weiterer Forschungsbedarf 93

3.3 Zielsetzung der vorliegenden Untersuchung 95

4. Methode 101

$\begin{array}{ll}\text { 4.1 Stichprobe und Rahmenplan } & 101\end{array}$ 
4.2 Versuchsanforderungen und -durchführung

4.2.1 Semantische Organisationsaufgabe („Sort-Recall“-Aufgabe)

4.2.2 Fragebogen zur Erfassung des aufgabenspezifischen Metagedächtnisses

4.2.3 Gedächtnisspannen-Aufgabe

4.2.4 Aufgaben zur Erfassung des Selbstkonzeptes und der Motivation

4.2.5 Aufgaben zur Erfassung der verbalen Fähigkeiten

5. Ergebnisse

5.1 Beobachtungen zum Phänomen des Nutzungsdefizits

5.1.1 Ineffektives kategoriales Organisieren

5.1.2 Ineffektiver bewusster Einsatz der kategorialen Organisationsstrategie

5.1.3 Verhaltenskonsistenz über zwei vergleichbare Aufgaben

5.1.4 Zusammenfassung der Ergebnisse zum Phänomen des Nutzungsdefizits

5.2 Determinanten für effektives kategoriales Organisieren

5.2.1 Altersunterschiede bei kognitiven und motivationalen Faktoren

5.2.2 Determinanten für effektives kategoriales Organisieren

5.2.3 Determinanten für einen effektiven bewussten Einsatz der kategorialen Organisationsstrategie

5.2.4 Zusammenfassung

5.3 Exkurs: Geschlechtsspezifische Besonderheiten

6.1 Antworten auf die zentralen Fragestellungen

6.2 Einordnung der eigenen Befunde in die empirische Datenlage

6.3 Bedeutung der Befunde vor dem Hintergrund der Strategie-Emergenz-Theorie 


\section{Vorwort}

Die vorliegende Arbeit entstand im Rahmen eines umfangreichen Forschungsprojektes, an dessen Gelingen bis heute zahlreiche Personen mitwirken. Ich möchte mich daher an dieser Stelle zuerst bei den Eltern und Kindern für kontinuierliche Teilnahme und das große Interesse bedanken, mit dem sie die Studie begleiten.

Den entscheidenden Impuls zur Bearbeitung dieses Themas ging von Prof. Dr. Marcus Hasselhorn aus, dem ich für die intensiven Diskussionen danken möchte, die die vorliegende Arbeit nachdrücklich geprägt haben.

Mein besonderer Dank gilt weiterhin dem gesamten Projekt-Team, ohne das das Ganze nicht möglich gewesen wäre. Dazu gehören einmal die studentischen Hilfskräften, die in allen Phasen des Projektes sowohl bei der Erhebung und der Auswertung der Daten als auch bei der Betreuung der Kinder außergewöhnliches Engagement zeigten. Stellvertretend für alle, die zu verschiedenen Zeiten in dem Projekt tätig waren, seien hier die aktuellen Mitarbeiter genannt: Anna Grohmann, Carolin Schwiening, Isabel de Nocker, Jenny Schmidt, Katharina Brand, Natalie Neumann, Sibylle Teufert und Stefanie Vogt. Eine große Unterstützung ging auch von meinen Diplomandinnen Angelika Clausner, Silke Dräger, Jennifer Gaßmann, Linda Jost, Wiebke Mund und Jasmin Warwas aus. Darüber hinaus danke ich Herrn Böhlken für die technische Unterstützung.

Großer Dank gebührt auch Michael Lingen, mit dem ich die ganze Zeit über die Ziele dieses Projektes verfolgt habe. Darüber hinaus hat der konstruktive Austausch mit Prof. Dr. Wolfgang Schneider, Kristin Krajewski, Veronika Kron und Michael Hünnerkopf wesentliche Impulse für diese Arbeit geliefert.

Mein Dank gilt weiterhin Dagmar Breuker und meinen Kollegen, auf deren Kenntnisse und Fähigkeiten ich in den unterschiedlichen Phasen der Entstehung der Arbeit zurückgreifen durfte. Besonders bedanken möchte ich mich hier bei Cora Titz, die durch die Korrektur der Arbeit und ihre Anregungen eine große Hilfe war.

Widmen möchte ich dieses Buch meinem Mann Thomas, der mir die ganze Zeit in jeder Beziehung zur Seite stand. 


\section{Einleitung}

Es ist schon beeindruckend, wie gut wir uns in unserem Leben zurechtfinden. In einem großen Kaufhaus mit unzähligen Artikeln sind wir in kurzer Zeit in der Lage, genau das zu finden, was wir wollen. Eine Fähigkeit, die von kleinen Kindern mit großen Augen bewundert wird - haben sie doch die Erfahrung gemacht, dass es selbst in ihrem kleinen Kinderzimmer schier unmöglich erscheint ein bestimmtes Spielzeug wieder zu finden, wenn sie es brauchen. Noch wissen sie nicht, dass die Person, die sie beim Einkaufen gerade so bewundern bei dieser Aufgabe ebenso kläglich scheitern würde wie sie selbst. Aber was ist so anders daran? Spontan würde man als Antwort erwarten: „Weil das Kaufhaus aufgeräumt ist, dein Kinderzimmer aber nicht." Was aber steht hinter diesem Konzept, dass Aufräumen und Ordnung halten uns dabei hilft, uns zurechtzufinden? Wir haben im Laufe unseres Lebens gelernt, wie wir unsere Umwelt hierarchisch ordnen können. Dazu gehört, dass wir Ober- und Untergruppen bilden können, denen sich einzelne Dinge zuordnen lassen. Im Kaufhaus bedeutet dies, dass wir, wenn wir einen Becher Joghurt suchen, zuerst in die Lebensmittelabteilung und dort in den Gang mit den Milchprodukten gehen.

Diese Logik würde sehr kleinen Kindern eventuell noch einleuchten, etwas ältere Kinder hätten aber wahrscheinlich ihre Zweifel. So haben sie in Kaufhäusern beispielsweise auch schon die Erfahrung gemacht, dass Schokolade sowohl bei den Süßigkeiten, als auch Backzutaten, Weihnachtsartikeln und direkt an der Kasse zu finden ist. Eine Problematik, mit der Erwachsene anscheinend gut zurechtkommen.

Festzuhalten bleibt, dass jüngere Kinder und Erwachsene einerseits sowie ältere Kinder andererseits auf verschiedene Art und Weise an derartige Anforderungen herangehen. Unklar bleibt hingegen, woran es liegt, dass kategoriale Ordnungskriterien in unterschiedlichem Ausmaß genutzt werden. Haben Kinder einfach eine andere Sicht der Welt und kategoriale Strukturen werden sich ihnen erschließen, sobald sie die Welt mit den Augen der Erwachsenen sehen? Wurde ihnen nicht ausreichend erklärt, wie derartige implizite Ordnungskriterien genutzt werden können? Müssen sie ihre eigenen Erfahrungen sammeln und verschiedene Vorgehensweisen ausprobieren, bevor sie sich für eine bestimmte Herangehensweise an solche Anforderungen entscheiden?

Diese Fragen zur Entwicklung von kategorialen Organisationsprozessen gehören bereits seit dem Ende der 60er Jahre $\mathrm{zu}$ den zentralen Themen der entwicklungspsychologischen Gedächtnisforschung (für einen Überblick siehe Schneider, 2000). Aktuell sind sie bis heute geblieben, auch wenn in der Zwischenzeit bereits viele 
Erkenntnisse zu diesem Thema gewonnen werden konnten (z.B. Hasselhorn, 1996; Sodian \& Schneider, 1999; Schlagmüller \& Schneider, 2002). Dazu gehört, dass es sich beim kategorialen Organisieren um eine Fähigkeit handelt, die sich normalerweise im Laufe der Grundschulzeit entwickelt. Dabei scheint es allerdings diverse Unterschiede in der Effektivität des Verhaltens und dem Zeitpunkt des erstmaligen Strategiegebrauchs zu geben. Eine ausbleibende Leistungsdienlichkeit des Verhaltens wurde in diesem Zusammenhang als Nutzungsdefizit interpretiert. Neben der Beschreibung der Differenzen wurde auch nach Bedingungen und Ursachen gesucht. Bei den in diesem Zusammenhang diskutierten Einflussfaktoren handelt es sich auf der einen Seite um basale kognitive Prozesse (z.B. Informationsverarbeitungsgeschwindigkeit) und auf der anderen Seite um Facetten der Wissensbasis, zu denen sowohl metakognitives als auch bereichsspezifisches Wissen gehören (vgl. Bjorklund, 2000; Hasselhorn, 1995, 1996; Schneider, 2000).

Die vorliegende Arbeit beschäftigt sich mit den Fragen, ob sich das Phänomen des Nutzungsdefizits auch im intraindividuellen Verlauf finden lässt und in welchem Ausmaß unterschiedliche intraindividuelle Entwicklungsverläufe beim kategorialen Organisieren auftreten. Darüber hinaus soll exploriert werden, welche Gründe es für die geringe Effektivität der Strategieproduktion und die unterschiedlichen Entwicklungsverläufe gibt. Dafür werden in Kapitel 2.1 die bisherigen Annahmen zur Strategieentwicklung und in Kapitel 2.2 die Verfahren zur Überprüfung kategorialer Organisationsprozesse vorgestellt. Im Anschluss daran wird erläutert, welche Annahmen bisher in Bezug auf die Ursachen strategischen Verhaltens formuliert wurden (Kapitel 2.3) und welchen Determinanten ein besonderer Stellenwert für die Strategieproduktion und -effektivität zugesprochen wird (Kapitel 2.4). Die Zielsetzung der vorliegenden Arbeit wird in Kapitel 3 detailliert erläutert, woran sich eine Beschreibung der konkreten Umsetzung anschließt (Kapiel 4). Die Ergebnisse umfassen einmal die Beobachtungen zum Phänomen des Nutzungsdefizits (Kapitel 5.1) und außerdem die Befunde zu Determinanten des effektiven Strategiegebrauchs (Kapitel 5.2). Eine zusammenfassende Diskussion, bei der die zentralen Fragestellungen noch einmal aufgegriffen und die vorliegenden Ergebnisse vor dem Hintergrund anderer empirischer Befunde und Erklärungsmodelle (Strategie-Emergenz-Theorie) diskutiert werden, enthält Kapitel 6. 


\section{Theoretischer und empirischer Hintergrund}

\subsection{Annahmen zur effektiven Strategieentwicklung}

Bevor auf die Effektivität von Strategien und ihre Entwicklung eingegangen wird, ist es wichtig sich damit auseinanderzusetzen, wodurch sich strategisches Verhalten von unstrategischem Verhalten abhebt, das sich unter Umständen ähnlich äußert.

\subsubsection{Strategisches Verhalten}

Der Einsatz von Strategien bei der Bearbeitung von Gedächtnisanforderungen ist bereits seit der kognitiven Wende in den 60er Jahren ein wichtiges Thema in der Kognitionsund Entwicklungspsychologie und besitzt nach wie vor Aktualität (Bjorklund \& Miller, 1997; Bower, 2000; Siegler, 1996, 2000, 2001). Damals sprach man davon, dass man einen „Plan“ zur Steuerung und Koordination des Verhaltens benötigen würde (Miller, Galanter \& Pribram, 1960, S.211 ff.). Dieser Begriff wurde später von dem heute gebräuchlicheren Strategiebegriff abgelöst. Im Folgenden soll der hier verwendete Strategiebegriff näher definiert werden.

Zur Definition einer Strategie wurden bisher unterschiedliche Kriterien aufgestellt (Bjorklund \& Harnishfeger, 1990; Brown, 1978; Flavell, 1970; Kail, 1979; Paris, Lipson \& Wixton, 1983; Pressley, Forrest-Pressley, Elliott-Faust \& Miller, 1985; Siegler \& Jenkins, 1989). Weitgehende Übereinstimmung herrscht darüber, dass strategisches Verhalten über die obligatorischen Konsequenzen der Aufgaben („normales“ Lernverhalten, d.h. Aufmerksamkeit und Beschäftigung mit den Anforderungen) hinausgehen und zielgerichtet erfolgen muss (vgl. Bjorklund, 1990; Schneider \& Pressley, 1989; Siegler \& Jenkins, 1989). Andere Kriterien wie Spontaneität, Selektivität, Intentionalität, Bewusstheit, Kontrolliertheit und die Kapazitätsbeanspruchung wurden hinsichtlich ihrer Bedeutung kontrovers diskutiert (Brown, Bransford, Ferrara \& Campione, 1983; Pressley, Ross, Levin \& Ghatala, 1985; Siegler \& Shrager, 1984). Jedes Kriterium konnte in Abhängigkeit von der Betrachtungsebene und konkreten Zielsetzung eine besondere Bedeutung bekommen oder auch verlieren. Will man die Anfänge strategischer Kompetenzen untersuchen, so ist man darauf angewiesen, ein niedriges Schwierigkeitsniveau $\mathrm{zu}$ schaffen, was z.B. durch die Gestaltung stark unterstützender Bedingungen (z.B. durch spezifische Instruktionen) geschehen kann. Das Kriterium der Spontanität beim Strategieeinsatz rückt damit in den Hintergrund. Wenn bei 
einer Problemstellung keine gleichwertigen Strategien nebeneinander existieren oder nur eine bestimmte Reaktion unterdrückt werden soll, so entfällt die Notwendigkeit der Selektion. Neben diesen aufgabenbezogenen Kritikpunkten können auch personenbezogene Kompetenzen die Bewertung strategischer Fähigkeiten erschweren. Bei der Beurteilung der Intentionalität und Bewusstheit ist man in entwicklungspsychologischen Untersuchungen auf Selbstaussagen der Kinder angewiesen. Wenn ein Kind sein Verhalten in der konkreten Situation nicht begründen kann, so sind verschiedene Ursachen denkbar. Bei sehr geübten Verhaltensweisen könnte beispielsweise schon ein Automatisiertheitsgrad erreicht worden sein, der die Verbalisierung der zugrunde liegenden Motive erschwert. Die durch zunehmende Erfahrung erreichte Automatisierung von Verhaltensweisen senkt auch das Ausmaß an kognitiver Belastung beim Strategieeinsatz und die Notwendigkeit der Kontrolle des gezeigten Verhaltens. Je nachdem welche Aspekte strategischer Kompetenzen untersucht werden sollen, bekommen die beschriebenen Kriterien ein stärkeres oder schwächeres Gewicht (vgl. Tabelle 2.1).

Tabelle 2.1: Definitionskriterien für Strategien und ihre Bedeutung für empirische Überprüfungen

\begin{tabular}{|c|c|c|c|}
\hline $\begin{array}{l}\text { Allgemeine } \\
\text { Zuordnung }\end{array}$ & Kriterium & $\begin{array}{l}\text { Wichtig bei } \\
\text { Untersuchung von }\end{array}$ & $\begin{array}{l}\text { Schwierig bei Untersuchung } \\
\text { von }\end{array}$ \\
\hline \multirow[t]{2}{*}{$\begin{array}{l}\text { Eigen- } \\
\text { initiative }\end{array}$} & Spontaneität & $\begin{array}{l}\text { Selbständigem } \\
\text { Strategiegebrauch }\end{array}$ & $\begin{array}{l}\text { anfänglichem Strategiegebrauch } \\
\text { (Unterstützung notwendig) }\end{array}$ \\
\hline & Selektivität & $\begin{array}{l}\text { Auswahl zwischen } \\
\text { gleichberechtigten } \\
\text { Strategien }\end{array}$ & $\begin{array}{l}\text { Unterdrückung einer best. } \\
\text { Reaktion }\end{array}$ \\
\hline \multirow{2}{*}{$\begin{array}{l}\text { Bewusstheit / } \\
\text { Zielgerich- } \\
\text { tetheit }\end{array}$} & \begin{tabular}{|l|} 
Intentionalität \\
Bewusstheit \\
\end{tabular} & \multirow{2}{*}{$\begin{array}{l}\text { Metakognitiven } \\
\text { zentral-exekutiven } \\
\text { Kompetenzen }\end{array}$} & $\begin{array}{l}\text { Sehr geübten (automatisierten) } \\
\text { Verhaltensweisen }\end{array}$ \\
\hline & Kontrolliertheit & & $\begin{array}{l}\text { Kindern mit geringen verbalen } \\
\text { Kompetenzen oder geringer } \\
\text { Motivation }\end{array}$ \\
\hline Anstrengung & $\begin{array}{l}\text { Kapazitäts- } \\
\text { beanspruchung }\end{array}$ & $\begin{array}{l}\text { Schwierigkeitsniveau } \\
\text { einer Strategie }\end{array}$ & Sehr einfachen Anforderungen \\
\hline
\end{tabular}


Für eine allgemeine Strategiedefinition können die hier dargestellten Merkmale nicht gleichberechtigt nebeneinander stehen. Eine spezielle Gewichtung ist allerdings nur unter Berücksichtigung der konkreten Fragestellung, der untersuchten Stichprobe und realisierten Rahmenbedingungen möglich. Ganz allgemein muss entschieden werden, wie viel Eigeninitiative erwartet wird (Spontanität, Selektivität), wie anstrengend die Anforderung für das Kind sein soll (Kapazitätsbeanspruchung) und wie bewusst bzw. zielgerichtet das Verhalten ablaufen soll (Intentionalität, Kontrolliertheit).

Die Strategiedefinition von Hasselhorn (1996) schafft die notwendige Flexibilität, indem zwei übergeordnete Kriterien wahlweise durch Einbeziehung der anderen Merkmale ergänzt werden.

„Strategien werden als Prozesse aufgefasst, die auf ein Lern- oder Behaltensziel ausgerichtet sind und die mehr als die obligatorischen Konsequenzen der Bearbeitung einer Gedächtnisanforderung darstellen (notwendige bzw. obligatorische Merkmale). Darüber hinaus müssen sie wenigstens eine zusätzliche akzessorische Eigenschaft aufweisen. Als akzessorische Eigenschaften kommen in Frage, dass sie intentional, bewusst, spontan, kontrolliert, kapazitätsbelastend und/oder selektiv sind“(Hasselhorn, 1996, S. 61).

Im Umgang mit Gedächtnisaufgaben sind sowohl in der Lern- und Behaltensphase als auch beim Erinnern in der Abrufphase verschiedene Formen von strategischem Verhalten denkbar. Lerninhalte können beispielsweise wiederholt nachgesprochen ( bedeutungshaltigen Erinnerungshilfen verknüpft (Elaboration) werden (für eine Übersicht vgl. Knopf \& Schneider, 1998; Schacter, Wagner \& Buckner, 2000; Schneider \& Bjorklund, 1998).

Das Wiederholen von zu lernenden Elementen (Rehearsal) ist besonders beim Aneignen kontext-unabhängiger Inhalte (Wort-, Bild- oder Gegenstandslisten) geeignet und kann bereits im Vorschulalter eingesetzt werden (vgl. Flavell, Beach \& Chinsky, 1966; Gathercole, Adams \& Hitch, 1994; Guttentag, Ornstein \& Siemens, 1987; Ornstein \& Naus, 1985; Ornstein, Naus, Liberty, 1975). Die Erfassung solcher Strategien geschieht teilweise durch indirekte Verfahren, indem Rückschlüsse aus Lippenbewegungen (z.B. Flavell, 1970) oder speziellen Reproduktionsmustern (z.B.Hagen \& Kingsley, 1968) gezogen werden, oder durch direkte systematische Verhaltensbeobachtungen (Rundus, 1971) und den Angaben der Probanden (vgl. McGilly \& Siegler, 1990).

Der Einsatz von Elaborationsstrategien setzt eine reichhaltige Wissensbasis voraus und wird daher oft erst im Jugendalter beobacht (Beuhring \& Kee, 1987; Flavell, 1970). 
Untersuchen lässt sich die Generierung von visuellen oder verbalen Repräsentationen mit Hilfe von Aufgaben zum Paar-Assoziations-Lernen oder seinen Varianten (Kee, 1994; Pressley, 1982; Schneider \& Pressley, 1989).

In dieser Arbeit liegt der Fokus jedoch auf der Strategie des kategorialen Organisierens. Bei Organisationsstrategien geht es grundsätzlich darum, in die unübersichtliche Fülle des Lernmaterials Ordnung zu bringen. Dabei sind verschiedene Möglichkeiten des Ordnens bzw. Strukturierens denkbar. Gruppierungsprozesse können unabhängig von bedeutungshaltigen Merkmalen der Items erfolgen (primäres Organisieren). Wenn über die zu lernenden Inhalte ein bestimmtes Wissen vorliegt, können gemeinsame charakteristische und zeitüberdauernde Merkmale von Items (z.B. Lautähnlichkeit) oder thematische bzw. semantische Relationen genutzt werden (sekundäres Organisieren). Dabei kann zwischen subjektivem und kategorialem Organisieren unterschieden werden:

- Beim subjektiven Organisieren spiegelt sich die vom Probanden selbst erzeugte Struktur in der Art der Reproduktion (Erinnerungsreihenfolge) wider.

- Beim kategorialen Organisieren (Clustern) wird die einer Liste inhärente Struktur ausgenutzt, indem die Items nach Kategorien gruppiert gelernt und/oder wiedergegeben werden.

Das Ordnen nach Kategorien stellt ein wichtiges und weit verbreitetes Gliederungskriterium dar (vgl. Chin-Parker\& Brian, 2002; Kalish, 2002; Quinn \& Eimas, 2000). Die Anwendung dieser Strategie bei einer konkreten Aufgabenanforderung lässt sich dabei in verschiedene Phasen untergliedern (vgl. Miller \& Seier, 1994):

- $\quad$ Phase 1: Suche nach möglichen Vorgehensweisen für die Bewältigung der Anforderung

- $\quad$ Phase 2: Entdeckung und Bewertung der Strategie

- $\quad$ Phase 3: Anbahnung der Strategie

- $\quad$ Phase 4: Durchführung der Strategie

- $\quad$ Phase 5: Reflektion über das eigene Verhalten

- $\quad$ Phase 6: Beibehalten der Strategie

- $\quad$ Phase 7: Verarbeitung des zu behaltenden Materials.

Aussagen über strategische Kompetenzen und ihre Entwicklung wurden lange Zeit ausschließlich auf der Basis der vierten Phase getroffen. Beobachtet wurde also vorrangig das direkt beobachtbare Verhalten, das bei Anwendung einer bestimmten Strategie gezeigt werden sollte. Erst in den letzten Jahren rückte das Bewusstsein für die Vielschichtigkeit 
strategischer Gedächtnisprozesse in den Mittelpunkt des Forschungsinteresses (vgl. Bjorklund, 2000; Lockard, 2000, Siegler, 2000; Tulving, 2000).

\subsubsection{Entwicklung kategorialen Organisationsverhaltens}

Kennzeichnend für die Entwicklung von Strategien ist einmal der Zeitpunkt, an dem das relevante Verhalten bzw. die intendierte Strategie das erste Mal beobachtet werden kann. Darüber hinaus lässt sich an dem weiteren Verlauf ablesen, ob es sich bei diesem Verhalten um ein vorübergehendes Ereignis oder ein stabiles Phänomen handelt.

\section{Zeitpunkt der erstmaligen Strategieanwendung}

Frühe strategische Kompetenzen, z.B. im Umgang mit räumlichen Anordnungen, lassen sich bereits bei Zwei- bis Vierjährigen beobachten (DeLoache, Cassidy \& Brown, 1985). Insgesamt ist der Strategieeinsatz im Vorschulalter allerdings sehr störanfällig und daher an spezielle Rahmenbedingungen geknüpft (vgl. Blair, Perlmutter \& Myers, 1978; Loughlin \& Daehler, 1973; Sophian \& Wellman, 1983; Ratner \& Myers, 1980) und auf einzelne Bereiche beschränkt (vgl. Brown, Bransford, Ferrara \& Campione, 1983).

Hinsichtlich des Zeitpunkts, an dem zum ersten Mal das Sortieren von kategorisierbarem Material gelingt, gibt es unterschiedliche Ansichten. Insgesamt gelingt es Kindern zu Beginn der Grundschulzeit nur selten, diese Strategie einzusetzen. Während Bjorklund $(1985,1987)$ aber davon ausgeht, dass ein bewusster, zielgerichteter Gebrauch der kategorialen Organisationsstrategie erst ab dem 13. Lebensjahr möglich ist, postuliert Hasselhorn (1996) den Erwerb dieser Strategie bis zum Ende der Grundschulzeit (mit 8-10 Jahren).

Die Beobachtung, dass Organisationsstrategien sich etwas später entwickeln als z.B. Wiederholungsstrategien (vgl. Moely et al., 1969), lässt sich damit erklären, dass die Entdeckung semantischer Relationen zwischen Items einen wesentlich komplexeren und anstrengenderen Prozess darstellt. Differenziert man darüber hinaus zwischen den Phasen, in denen bei der Aufgabenbearbeitung auf kategoriale Strukturen zurückgegriffen wurde (beim Lernen oder beim Erinnern des Lernmaterials), so zeigen sich altersbezogene Unterschiede: Während Zweit- und Sechstklässler diese Strategie wesentlich häufiger in der Lernphase einsetzen, ist der Strategiegebrauch bei Studenten in allen Phasen der Aufgabenbearbeitung gleich gut (Kee \& Bell, 1981). 


\section{Verlauf der Strategieentwicklung}

Hinsichtlich des Verlaufs der Strategieentwicklung gibt es unterschiedliche Ansichten. Während einige Forscher von einer kontinuierlichen Verbesserung der strategischen Fähigkeiten ausgehen (z.B. Flavell, Miller \& Miller, 1993), postulieren andere, dass diesen Beobachtungen ein abrupter Kompetenzerwerb zugrunde liegt und sich der Entwicklungsprozess sprunghaft vollzieht (z.B. Sodian \& Schneider, 1999). Berücksichtigt man dabei die Vielfalt an strategischen Verhaltensmöglichkeiten, die es in den verschiedenen Entwicklungsstufen gibt, so zeigt sich die Komplexität der Entwicklung strategischer Kompetenzen.

\section{Linearer Verlauf (Stufenmodell)}

Bei allem Bewusstsein für die intra- und interindividuelle Variabilität des strategischen Verhaltens (vgl. Folds, Footo, Guttentag \& Ornstein, 1990), gibt es ein Bedürfnis nach einem allgemeinen Entwicklungsmodell für die Entwicklung des strategischen Verhaltens. Ausgehend von der Mediationstheorie (vgl. Kendler \& Kendler, 1962), wurde von einer kontinuierlichen Verbesserung der strategischen Fertigkeiten ausgegangen und ein linearer Entwicklungsverlauf unterstellt. In der Phase des Mediationsdefizits produzieren Kinder in der Regel keine Strategien und profitieren bei der Aufgabenbearbeitung nicht von Hilfestellungen (Reese, 1962). Die daran anschließende Phase, in der die Strategieanwendung nicht spontan erfolgt, sondern noch konkreter Hilfestellungen bedarf, wird als Produktionsdefizit bezeichnet (Flavell, 1970).

Abbildung 2.1 gibt einen Überblick über die Entwicklung des Kategorisierungsverhaltens nach Moely et al. (1969). Als Index für das Organisationsverhalten wurde der „Ratio of Repetition“ (RR) herangezogen, der von der Arbeitsgruppe von Bousfield (Bousfield \& Bousfield, 1966) für symmetrisch konstruierte Listen (gleiche Anzahl von Items pro Kategorie) entwickelt wurde. Der Wertebereich des RR liegt zwischen 0 und 1, wobei hohe Werte ein hohes Maß an kategorialer Organisation anzeigen. Der Wert 1 kann nur erreicht werden, wenn alle Items aus einer Kategorie stammen. Um auszuschließen, dass der ermittelte RR-Wert nicht auf zufällige Gruppierungsprozesse zurückzuführen ist, wird ein Zufallswert bestimmt, der in Abhängigkeit von der Anzahl der Items und der verwendeten Kategorien variiert. Das Grundmodell des RR lässt sich als 
Zufallsanordnung der dargebotenen Items mit anschließender Zufallsanordnung darstellen (vgl. Pellegrino, 1975).

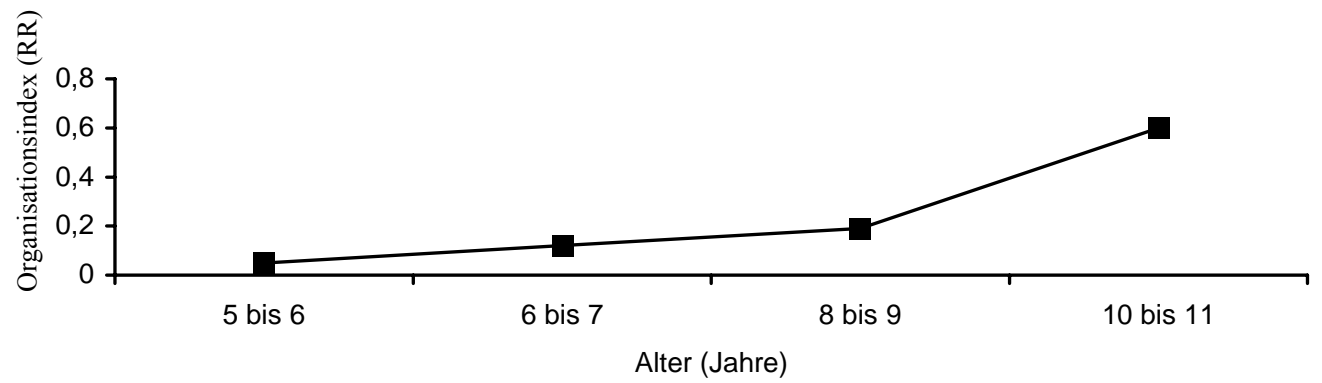

Abbildung 2.1: Entwicklung des Kategorisierungsverhaltens nach Moely et al. (1969)

Aus Abbildung 2.1 wird ersichtlich, dass Kinder erst nach dem 8. Lebensjahr anfangen, systematisch nach Oberbegriffen zu organisieren. Das Ausmaß an kategorialer Ordnung scheint mit zunehmendem Alter kontinuierlich zuzunehmen, woraus Rückschlüsse auf einen gleichmäßigen Entwicklungsprozess der Kategorisierungsstrategie gezogen wurden.

Die aus den Gruppendaten gezogenen Schlussfolgerungen müssen allerdings nicht mit den individellen Entwicklungsverläufen übereinstimmen. Sodian und Schneider (1999) konnten bei weniger als 10\% ihrer Probanden eine graduelle Verbesserung des strategischen Verhaltens beobachten. Für 80\% der Kinder vollzog sich der Strategie-Erwerb im Sinne eines „Alles-oder-nichts-Vorgangs“, wobei die „Sprünge“ zu unterschiedlichen Zeitpunkten erfolgen konnten.

\section{Sprunghafter Verlauf}

Sodian und Schneider (1999) beschrieben aus diesem Grund den Strategieerwerb bei kategorialen Organisationsanforderungen als einen sprunghaften Prozess. Belege für eine kontinuierliche Verbesserung des strategischen Verhaltens und der Leistung, wie sie lange Zeit auf der Basis von Querschnittdaten unterstellt worden war, konnten sie in ihrer Längsschnittstudie nicht finden. Aufgrund der Gruppenmittelwerte wären diese Annahmen zwar nahe liegend gewesen, sie ließen sich im intraindividuellen Verlauf allerdings nicht bestätigen. Ein entsprechendes Muster konnte nur bei wenigen Kindern gefunden werden. Trotz hoher Kurzzeitstabilitäten (über 2-3 Wochen) erwiesen sich die 2-, 4- oder 6Jahresstabilitäten für Organisationsverhalten und die Reproduktionsleistung als äußerst gering. Die einzige Ausnahme bildete die Clusterleistung zwischen dem 10. und 12. Lebensjahr. 
Insgesamt waren die individuellen Stabilitäten des strategischen Verhaltens und der Abrufleistung deutlich geringer als es anhand der Querschnittbefunde zu erwarten gewesen wäre (Schneider \& Sodian, 1997; Sodian \& Schneider, 1999). Die postulierten graduellen Leistungssteigerungen waren nur bei $8 \%$ der untersuchten Kinder zu beobachten. $80 \%$ der Kinder schafften zwischen zwei Messzeitpunkten einen Sprung vom Zufallsniveau zu annähernder Perfektion, während 8\% der Kinder sich von Anfang an strategisch verhielten. Die Variabilität betraf nicht nur den Entwicklungsverlauf, sondern auch den Zeitpunkt der erstmaligen Anwendung einer Strategie. Bei vielen Kindern, die bereits einen frühen Strategieeinsatz gezeigt hatten, kam es zwischenzeitlich zur Aufgabe dieser Strategie sowie zu ihrer Wiederentdeckung zu einem späteren Zeitpunkt.

Auch Schlagmüller und Schneider (2002) beobachteten in ihrer mikrogenetischen Studie einen eher abrupten Erwerb einer Sortierstrategie und nur in wenigen Fällen eine kontinuierliche Verbesserung. Dieses Muster ließ sich allerdings nicht auf die Entwicklung der kategorialen Organisationsstrategie in der Abrufphase übertragen. Dort fielen die Veränderungen weniger deutlich aus als in der Lernphase. Demnach scheint es sich bei beiden Prozessen (Nutzen kategorialer Strukturen bei der Informationsenkodierung und beim Informationsabruf) um unterschiedliche Komponenten zu handeln.

\section{Verlauf beim multiplen Strategiegebrauch: Overlapping-Waves-Model}

Neben der Anwendung der hier zu betrachtenden kategorialen Organisationsstrategie können bei der Bearbeitung einer Sort-Recall-Anforderung auch andere Strategien, wie das Rehearsal und Elaborationen eingesetzt werden (vgl. Cho \& Ahn, 2003), wie unter Kapitel 2.1 kurz aufgegriffen. Das von Siegler (1996) entwickelte Overlapping-Waves-Model (Abbildung 2.2) stellt eine realistischere und vor allem im Bereich arithmetischer Strategien gut untersuchte Alternative zum Stufenmodell (Kendler \& Kendler, 1962; Reese, 1962) dar.

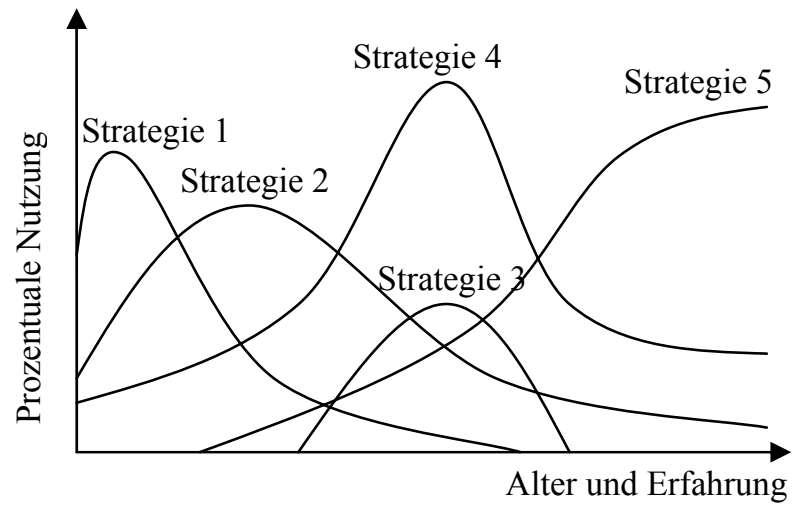

Abbildung 2.2: Schematische Darstellung des Overlapping-Waves-Modells (aus: Siegler, 1996). 
In dem Modell wird davon ausgegangen, dass einem Kind $\mathrm{zu}$ einem bestimmten Zeitpunkt nicht nur eine, sondern mehrerer Strategien zur Verfügung stehen (vertikale Perspektive; „overlapping“), welche zu unterschiedlichen Anteilen genutzt werden. Zum anderen wird angenommen, dass eine Strategie nicht abrupt zugunsten einer anderen verworfen wird, sondern deren Anwendungshäufigkeit in Abhängigkeit von Alter und Erfahrung mit einer wellenförmigen Linie beschreibbar ist (horizontale Perspektive, „waves“). Strategien werden demnach entdeckt, auf bestimmte Aufgabenbereiche angewendet und zunehmend eingeübt. Gleichzeitig konkurrieren verschiedene Strategien miteinander, so dass weniger nützliche mit zunehmendem Alter und steigender Erfahrung wieder allmählich in ihrer Häufigkeit absinken (Siegler, 1996). Nach diesem Modell sind Kinder nie „strategielos“, sondern zeigen Ansätze von planvollem, zielgerichtetem Verhalten (z.B. Sophian \& Wellman, 1983), das sich qualitativ allerdings von „reiferen“ Strategien unterscheidet.

Hinsichtlich der Kriterien, nach denen bei einer bestimmten Aufgabe eine der vielen verfügbaren Strategien ausgewählt wird, gehen Siegler und Schrager (1984) von einer adaptiven Vorgehensweise aus. Die Anfangsphase wird durch Verwendung von allgemeinen, bewährten Strategien geprägt, wobei unter Umständen effektivere, aber wenig erprobte Strategien vernachlässt werden. Dieses würde als Produktionsdefizit interpretiert werden. Mit zunehmender Häufigkeit der Aufgabenbearbeitung werden weitere Strategien ausprobiert und eingeübt, um die effektivste und effizienteste herauszufinden. Diese Vorgehensweise könnte einen Teil der im Strategiegebrauch aufgefundenen Instabilität erklären.

\subsubsection{Entwicklung einer effektiven kategorialen Organisationsstrategie}

Der Entwicklungsprozess ist nicht damit abgeschlossen, dass die kategoriale Organisationsstraegie angewendet werden kann. Wie bereits in Abschnitt 2.1.1 beschrieben, ist ein entscheidendes Merkmal von Strategien, dass sie auf ein Lern- oder Behaltensziel ausgerichtet sind (vgl. Hasselhorn, 1996). Eine solche Effektivität ist in der Vergangenheit für verschiedene Strategien auch durchaus belegt worden. Die Beobachtung, dass häufiges Wiederholen mit einer besseren Behaltensleistung einherging, interpretierten Flavell und seine Kollegen (1966) als Hinweis auf einen effektiven Einsatz der Rehearsalstrategie. Auch wenn die Befunde von verschiedenen Autoren (z.B. Liberty \& Ornstein, 1973) diese Ansicht stützten, muss die Leistungsdienlichkeit des Rehearsals in Abhängigkeit von bestimmten Einflussfaktoren betrachtet werden. Eine besondere Rolle scheint dabei die Qualität der 
Strategieproduktion zu spielen (Naus, Ornstein \& Aivano, 1977; Ornstein \& Naus, 1983), wobei auch das Alter der Anwender der Strategie berücksichtigt werden muss (Ornstein \& Naus, 1978, 1985).

Das Einüben von Elaborationsstrategien kann ebenfalls eine deutliche Leistungsverbesserung nach sich ziehen (Pressley, 1982). Das Ausmaß der Strategieeffektivität hängt allerdings stark von dem Alter der Strategieanwender ab (vgl. Reese, 1976b).

Darüber hinaus konnte auch die Effektivität des kombinierten Strategiegebrauchs belegt werden. Schlagmüller und Schneider (2002) fanden ebenso wie Coyle und Bjorklund (1997), dass der gleichzeitige Gebrauch von verschiedenen Strategien mit einer besseren Gedächtnisleistung zusammenhing. Allerdings war die Variabilität im Strategiegebrauch deutlich geringer als bei Coyle und Bjorklund (1997), die eine alterskorrelierte Zunahme hinsichtlich der Homogenität des Strategiegebrauchs beobachteten. Ausschlaggebend dafür dürfte sein, dass zumindest in der untersuchten Altersgruppe der Nutzen der untersuchten Strategien bei den jeweiligen Anforderungen sehr offensichtlich war und dadurch wenig Konkurrenz mit anderen Strategien herrschte. Auch aktuelle Studien belegten den Vorteil des kombinierten Einsatzes von Rehearsal und kategorialem Organisieren für die Behaltensleistung von Kindergartenkindern (Schneider, Kron, Hünnerkopf \& Krajewski, 2004).

In Bezug auf das alleinige kategoriale Organisieren gibt es ebenfalls eine Vielzahl von Befunden, die seine Effektivität bestätigen. So konnte häufig beobachtet werden, dass vermehrtes Organisationsverhalten zu einer Steigerung der Reproduktionsleistung führt (Hasselhorn, 1992; Schneider, 1986).

Die Tatsache, dass Kinder eine Strategie einsetzen können und dieses in einem konkreten Fall auch tun, bedeutet allerdings nicht zwangsläufig, dass dieses Verhalten ihnen auch nützt. Es existieren zahlreiche Studien, in denen der postulierte Zusammenhang zwischen Strategiegebrauch und Leistungssteigerung nicht gefunden wurde (Bjorklund, Coyle \& Gaultney, 1992; Lange, Guttentag \& Nida, 1990; Lange \& Pierce, 1992; Miller, DeMarieDreblow \& Woody-Ramsey, 1986). Diese Beobachtungen sind allerdings nicht neu. Seit der Thematisierung des Produktionsdefizits (Flavell, Beach \& Chinsky, 1966) gibt es Hinweise darauf, dass der Einsatz einer Strategie nicht bei allen Kindern gleich effektiv ist (Laurence, 1966). Auch andere Autoren (z.B. Reese, 1976, Waters, 1982) berichteten von dem Phänomen der geringen Strategieeffektivität bereits bevor dafür der Begriff des 
Nutzungsdefizits eingeführt wurde (DeMarie-Dreblow \& Miller, 1988; Miller \& Harris, 1988; Miller, DeMarie-Dreblow \& Woody-Ramsey, 1986; Woody-Ramsey \& Miller, 1988).

Über die entwicklungspsychologische Bedeutung der Verhaltenskonsequenzen der Strategieproduktion war bereits früher schon reflektiert worden (z.B. Flavell, 1970; Reese, 1976a, 1976b). Das für die Strategieproduktion aufgestellte Entwicklungsmodell, das nach der Überwindung des Mediations- und Produktionsdefizits bereits vom Erreichen des Reifestadiums ausgeht, schien den Anforderungen nicht mehr $\mathrm{zu}$ genügen, so dass eine Erweiterung um den Aspekt der Strategienutzung erforderlich wurde (Flavell, 1970). Aus der Erkenntnis, dass auch die Effizienz des Verhaltens in einem Entwicklungsmodell berücksichtigt werden müsste, leitete Reese (1976b) ab, dass auch eine Phase der ineffizienten Strategieproduktion in das Modell integriert werden müsste. Sein Vorschlag bestand darin, der Stufe des Produktionsdefizits eine Stufe der Produktionsineffizienz voranzustellen, in der die Strategie - wie auf der Stufe des Produktionsdefizits - unter entsprechender Anleitung angewendet wird, aber noch zu keiner Leistungssteigerung führt. Entsprechend dieser Annahme kann man das Entwicklungsstadium, in dem sich ein Kind gerade befindet, daran erkennen, ob unter entsprechender Instruktion das Verhalten überhaupt gezeigt wird (=Überwindung des Mediationsdefizits) und ob dieses Verhalten positive Auswirkungen auf die Behaltensleistung hat (=Überwindung der Produktionsineffizienz). Sobald das Verhalten von den Kindern selbständig eingesetzt wird (=Überwindung des Produktionsdefizits), wird ein effektiver Strategiegebrauch erwartet (Reifestadium), auch wenn Reese (1976b) einräumt, dass auch ein spontaner ineffektiver Strategieeinsatz denkbar ist. Die Tatsache, dass Strategieproduktion und -nutzen unabhängig von der Spontaneität der Strategieanwendung diskutiert werden, wirft weitere Fragen auf. Für ein allgemeines Entwicklungsmodell sollte geklärt werden, ob es bei der Strategieentwicklung

- $\quad$ zuerst zur Verbesserung der Effizienz und dann der Spontanität,

- $\quad$ zuerst zur Verbesserung der Spontanität und dann der Effizienz oder

- $\quad$ zur gleichzeitigen Verbesserung von Spontanität und Effizienz

kommt.

Dabei darf auch die Bedeutung der Vollständigkeit der Strategieproduktion nicht vernachlässigt werden. Miller (1990) postulierte ein aus 4 Phasen bestehendes Entwicklungsmodell, wonach sich der Strategieerwerb von einer Teilproduktion der Strategie über eine Produktion ohne Leistungsverbesserung vollzieht, bis am Schluss eine Leistungssteigerung erreicht wird. 
Zur Beschreibung der Phase des ineffizienten Strategiegebrauchs wurde von Miller (1990) das Konzept des 슨

"a transitional phase in strategy development when spontaneously producing a strategy results in no benefit for performance (e.g., recall ...), less benefit for older children, or even a decline in performance" (Miller, 1990, S. 167).

Ganz allgemein lässt sich ein Nutzungsdefizit also dadurch charakterisieren, dass der real aus dem Strategieeinsatz gezogene Vorteil hinter dem zu erwartenden bzw. theoretisch erreichbaren zurückbleibt.

Mit Bezug auf die Annahmen des Defizit-Modells (vgl. Flavell, 1971) setzt die Beobachtung dieses Phänomens die vorherige Überwindung des Mediations- und Produktionsdefizits voraus. In der Phase des Nutzungsdefizits setzen Kinder bestimmte Strategien spontan ein, erfahren dadurch aber bei der Aufgabenbearbeitung nur einen sehr geringen oder gar keinen Leistungszuwachs (vgl. Miller, 1990).

Waters (2000) kritisierte an der Definition des Nutzungsdefizits, dass sie sowohl den Nachweis des Mediationsdefizits („kein Nutzen“) als auch den der Strategieineffizienz (,geringer Nutzen“) enthält. Ihrer Ansicht nach trägt die Einführung dieses unspezifischen Entwicklungsstadiums nicht $\mathrm{zu}$ einer Verbesserung des Verständnisses der Strategieentwicklung bei, da für einen fehlenden Leistungsvorteil auch der Begriff des Mediationsdefizits herangezogen und ein im Vergleich zu erfahrenen Strategen geringerer Nutzen als Strategieineffizienz bezeichnet werden könne. Dieser Argumentation hält Miller (2000) entgegen, dass die gewählten Begriffe nicht unabhängig vom jeweiligen Kontext gesehen werden dürfen. Während sie das Mediationsdefizit im Zusammenhang mit Trainingsstudien sieht, in denen Kinder aufgrund ihrer kognitiven Unreife nicht von einer Strategie profitieren, hat das Nutzungsdefizit für sie einen deskriptiven Charakter und ist situationsspezifisch. Eine spontane oder halbspontane (angeleitete) trainierte Strategie verbessert danach nur bei einer bestimmten Aufgabe unter bestimmten Bedingungen nicht die Abrufleistung. Aber auch wenn ein Kind in einigen Aufgaben oder Strategien nutzungsdefizitär ist, muss es dieses bei anderen Aufgaben und Strategien nicht sein (vgl. Blöte, Resing, Mazer \& Van Noort, 1999).

Miller (2000) räumt allerdings ein, dass für die Betrachtung der Entwicklungsveränderungen von der vollen Strategieproduktion mit geringer 
Aufgabenleistung hin zu voller Strategieproduktion mit hoher Leistung kein theoretisches Rahmenwerk existiert, mit dessen Hilfe man die Effizienz des Strategiegebrauchs genauer bestimmen könnte. Darüber hinaus betont Miller (2000), dass im Hinblick auf den Verlauf des effektiven Strategiegebrauchs diverse intra- und interindividuelle Veränderungen denkbar sind. Im Entwicklungverlauf stellt die ausbleibende Leistungssteigerung infolge des ersten Strategieeinsatzes keinen notwendigen Schritt dar und nicht alle Kinder weisen nach einem anfänglichen Gebrauch einer Teilstrategie einen anfänglichen Leistungsabfall auf oder produzieren ein Nutzungsdefizit in einer Teilaufgabe (vgl. Miller \& Seier, 1994). Es ist ebenso möglich, dass Schritte ausgelassen oder so schnell durchlaufen werden, dass sie nicht beobachtet werden können. Es ist auch denkbar, dass einige Kinder zu einer früheren Phase zurückkehren, so wie Kinder, die in einigen Durchgängen von ihrer Strategie profitieren, in anderen Durchgängen, wenn die relevante Kategorie ausgetauscht wurde, aber ein Nutzungsdefizit zeigten (DeMarie-Dreblow \& Miller, 1988). Auch die zeitliche Quantifizierung des Phänomens ist äußerst schwierig und von einer Vielzahl interner und externer Faktoren abhängig. Im Allgemeinen geht Miller (2000) davon aus, dass ein vollständiges Ausbleiben des Nutzens einer angemessenen Strategie ein kurzes Entwicklungsphänomen sein kann, auf das eine wesentlich längere Entwicklungsphase folgt, in der die Strategie allmählich immer nützlicher wird.

Leider trägt aber auch die Einbeziehung von verschiedenen Stufen der StrategieInsuffizienz nicht dazu bei, dass das Konzept klarer wird und besser zu evaluieren ist (vgl. Schlagmüller \& Schneider, 2002). Eine begriffliche Differenzierung, wie sie z.B. in Form des Begriffs der Nutzungsineffizienz (Reese, 1976b) vorkommt, drückt mehr eine theoretische Haltung zu diesem Phänomen als eine inhaltliche Unterscheidung aus (vgl. Hasselhorn, 1996).

Eine zusätzliche Schwierigkeit ergibt sich aus dem unterschiedlichen Verständnis von den Entwicklungsphasen und ihren charakteristischen Merkmalen sowie zugrunde liegenden Mechanismen, was zu einem heterogenen Gebrauch der in diesem Zusammenhang verwendeten Begriffe geführt hat. Während ursprünglich davon ausgegangen wurde, dass eine Strategie in der Phase des Mediationsdefizits nicht produziert werden kann (vgl. Flavell, 1978), schließen einige Autoren (z.B. Miller \& Seier, 1994) diese Möglichkeit nicht aus. Die Anwendung von vermeintlich strategischem Verhalten sollte nach Ansicht von Miller und Seier (1994) bei den jüngeren Kindern zwar aufgrund von unbewusst ablaufenden Prozessen zustande kommen und mehr oder weniger zufällig initiiert worden sein, diese Möglichkeit spielt aber für die theoretischen Implikationen eine bedeutsame Rolle. Wenn Kinder bereits 
Erfahrungen mit einem bestimmten Verhalten sammeln können, so besteht auch eine gewisse Wahrscheinlichkeit, dass dieses Erfahrungswissen von den Kindern gespeichert und später wieder abgerufen wird. Vor dem Mediationsdefizit müsste demnach noch ein anderes Defizit überwunden werden, in dem kein strategisches Verhalten gezeigt werden kann, ein Verfügbarkeitsdefizit.

Die unzureichende inhaltliche und formelle Präzisierung der einzelnen Konzepte erschwert die eindeutige Zuordnung von beobachtbaren Verhaltensweisen zu den jeweiligen Phasen. Orientiert man sich an dem Kriterium der Spontaneität bzw. Selbständigkeit bei der Definition der Strategien, so dürften streng genommen keine Trainingsstudien in die Untersuchung der Strategieentwicklung mit einbezogen werden. Konzentriert man sich aber bei der Untersuchung der Entstehung von strategischem Verhalten ausschließlich auf Beobachtungen von spontanem Strategieeinsatz, so lassen sich Mediations- und Produktionsdefizite nicht voneinander unterscheiden. Aus dem fehlenden Einsatz von Strategien kann nicht geschlussfolgert werden, dass die Kinder nicht über die grundlegenden Fähigkeiten dafür verfügen, da sie diese möglicherweise unter günstigeren Rahmenbedingungen gezeigt hätten (z.B. unter Anleitung, nach einem Training). Die Kontextabhängigkeit strategischen Gedächtnisverhaltens sollte allerdings nicht einfach mit dem Stadium des Produktionsdefizits gleichgesetzt (z.B. Brown \& DeLoache, 1978), sondern differenziert betrachtet werden. Da die bisherigen Annahmen zur Strategieentwicklung eher deskripitiven Charakter hatten (Reese, 1976a) und keine angemessene Theorie zur Erklärung der Phänomene zur Verfügung stellten, plädierte Hasselhorn (1995) für einen reflektierten Umgang mit den verwendeten Begrifflichkeiten. Da das Vorliegen eines Defizits impliziert, dass spezielle Kompetenzen nicht vorhanden und damit ein bestimmtes Verhalten nicht gezeigt werden kann (vgl. Reese, 1976), sollte diese Bezeichnung nicht verwendet werden, um strategisches Verhalten zu kennzeichnen, das zwar ineffizient, aber immerhin produziert wird. Für geeigneter hält er dafür den Begriff der Ineffizienz oder geringen Effizienz.

Insgesamt scheint nicht nur die Qualität des strategischen Verhaltens, sondern auch das Ausmaß des zu erwartenden Nutzens dieses Verhaltens auf einem Kontinuum angesiedelt zu sein. Aus diesem Grund wurde auch versucht, die zunehmende Effizienz der Strategieproduktion $\mathrm{zu}$ operationalisieren. Die Beurteilung der Effizienz des ersten Strategieeinsatzes setzt eine konkrete Verhaltensänderung voraus, d.h. dass unstrategisches Verhalten von einer strategischen Aufgabenbearbeitung abgelöst wird. Dafür wurde meistens darauf geachtet, dass zu Beginn der Untersuchung keine bzw. nur zufällige Sortier- oder Clusterleistungen auftraten und später bedeutsame Strategiewerte erreicht wurden. Bei einer 
stärkeren Orientierung an relativen als an absoluten Kriterien sollten allerdings auch Veränderungen von überzufälligem, aber unvollkommenem strategischem Verhalten zu eloquenterem Strategiegebrauch berücksichtigt werden. Bjorklund und Coyle (1995) bezeichneten eine ausbleibende Leistungsverbesserung unter diesen Voraussetzungen als „Quasi-Nutzungsdefizit“". Dieses äußerte sich in ihren Sort-Recall-Aufgaben darin, dass ein bedeutsamer und stabiler Anstieg der Clusterleistung nicht mit einer besseren Wiedergabeleistung einherging.

\section{Die Überwindung des Nutzungsdefizits}

Miller und Seier (1994) gehen von einer nicht-linearen Beziehung zwischen der Strategieentwicklung und Reproduktionsleistung aus und postulieren auf dem Weg zum effektiven Strategiegebrauch verschiedene charakteristische Phasen (vgl. Abbildung 2.3.).

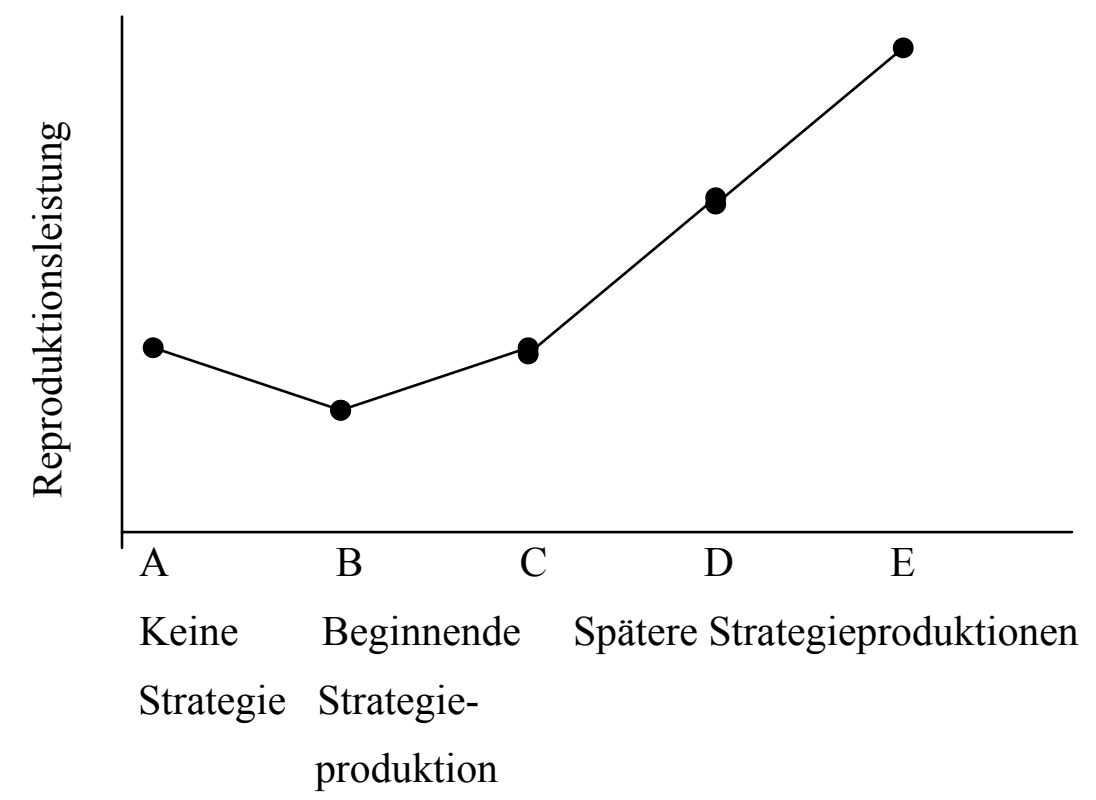

Abbildung 2.3.: Die Gedächtnisleistung als Funktion der Strategieentwicklung (aus: Miller \& Seier, 1994, S. 109)

Der erstmalige Strategiegebrauch kann ihrer Ansicht nach mit einem Abfall der Reproduktionsleistung einhergehen, besonders dann wenn viele Ressourcen in Anspruch genommen oder andere Aktivitäten unterbrochen werden müssen. Im Verlauf des weiteren Strategieeinsatzes äußert sich die Verbesserung darin, dass zuerst die Leistungseinbußen überwunden werden. Später wird die Reproduktionsleistung gesteigert, bis sie am Schluss das Niveau von älteren oder erfahreneren Strategen erreicht. Der durch den Strategiegebrauch zu erwartende Nutzen stellt sich demnach nicht kurzfristig, sondern erst langfristig ein (vgl. Miller \& Seier, 1994). Dieser Entwicklungsprozess kann je nach Art der Strategie in 
unterschiedlichen Altersstufen und mit unterschiedlicher Geschwindigkeit ablaufen. Theoretisch kann ein Nutzungsdefizit demnach im Verlauf von Monaten und Jahren, aber auch während der Absolvierung mehrerer Testdurchgänge überwunden werden. Um wirklich von einem Nutzungsdefizit sprechen zu können, sollte die Strategie allerdings so vollständig gezeigt werden, dass wirklich eine Leistungssteigerung erwartet werden kann.

Hinweise auf das Vorliegen eines Nutzungsdefizits lassen sich nach Miller und Seier (1994) an folgenden Befunden ablesen:

\section{- $\quad$ Altersdifferenzen:}

Ältere Kinder zeigen eine größere Strategieeffektivität als jüngere Kinder

- Defizit beim ersten Strategieeinsatz:

Eine spontan produzierte Strategie hilft anfänglichen Strategen nicht und kann sie sogar behindern

\section{- Defizit in der Anfangsphase:}

Die Behinderung der Wiedergabe durch den spontanen Strategiegebrauch betrifft nur die Anfangsphase. Mit zunehmender Erfahrung, d.h. in einem späteren Durchgang, steigt der Nutzen, der von dem Strategieeinsatz ausgeht

\subsection{Empirische Überprüfung kategorialer Organisationsprozesse}

Die bereits diskutierten Kriterien für strategisches Verhalten spielen auch bei der empirischen Überprüfung kategorialer Organisationsprozesse eine Rolle. Die grundsätzliche Entscheidung bei der Auswahl eines experimentellen Designs orientiert sich dabei an der Frage, ob man an dem realen Verhalten oder dem optimalen Fähigkeitsniveau der Kinder interessiert ist. Wenn man mit Fischer (1980) übereinstimmt, dass das wahre Entwicklungsniveau sich an der Obergrenze der Fähigkeiten einer Person zeigt, so sollte versucht werden, durch die Schaffung entsprechender Rahmenbedingungen möglichst nah an das optimale Fähigkeitsniveau der Kinder heranzukommen. Dieses kann u.a. durch entsprechende Instruktionen oder Trainingsinhalte erreicht werden.

Die systematische Analyse der Bedeutung kontextueller Rahmenbedingungen ermöglicht eine Ableitung von speziellen Verhaltensdimensionen, die an bestimmten Punkten 
des Entwicklungsverlaufs spezifische (strategische) Verhaltensänderungen bewirken (vgl. Ornstein et al., 1988). Das aktuelle Verhaltensniveau scheint danach weniger aussagekräftig zu sein als das optimale Verhaltensniveau. Hierbei gibt es unterschiedliche Ansichten darüber, wie massiv die Hilfestellungen ausfallen dürfen bzw. sollen, um dieses Niveau herzustellen (vgl. Furth \& Milgram, 1973; Hasselhorn, 1996; Kobasigawa \& Orr, 1973; Moely \& Shapiro, 1971; Moely \& Jeffrey, 1974). Die Bandbreite an Manipulationsmöglichkeiten reicht von lediglich indirekten Hilfestellungen durch die Aufgabenkonstellationen und den Versuchsleiter (Hasselhorn, 1996) über eine systematisch kategorial geordnete Itemdarbietung (z.B. Furth \& Milgram, 1973; Kobasigawa \& Orr, 1973; Moely \& Shapiro, 1971) bis zu einem direkten Strategietraining (z.B. Moely \& Jeffrey, 1974; Schuster-Böck \& Zoeke, 1991).

\subsubsection{Typische Aufgaben zur Überprüfung des kategorialen Organisierens}

Es gibt folglich viele Möglichkeiten, durch Schaffung spezieller Rahmenbedingungen bei der Aufgabendurchführung das Schwierigkeitsniveau der Anforderungen experimentell zu verändern, wenn das Ausmaß kategorialen Organisierens erfasst werden soll. In jeder Phase der Versuchsdurchführung besteht die Möglichkeit, das Anspruchsniveau bei der Aufgabenbearbeitung zu verändern, indem entweder mehr Selbständigkeit von den Kindern erwartet wird oder mehr kognitive Ressourcen beansprucht werden. Das Ausmaß an Intentionalität und Bewusstheit lässt sich durch eine Nachbefragung im Anschluss an die Aufgabenbearbeitung erfassen. Auch an dieser Stelle kann durch entsprechende Formulierung und die Gestaltung der Antwortmodalität (freie Begründung oder Entscheidung zwischen Antwortalternativen) das Schwierigkeitsniveau verändert werden.

Besonders häufig vertreten sind drei Aufgabentypen: Cued-Recall-Bedingungen, SortRecall-Bedingungen und Free Recall-Bedingungen. Das Ausmaß an Unterstützung nimmt dabei von Cued-Recall-Bedingungen über Sort-Recall-Bedingungen bis zu Free RecallBedingungen $\mathrm{ab}$.

Die Schaffung sehr unterstützender Bedingungen, die den Kindern den Einsatz einer bestimmten Strategie erleichtert, bietet sich an, wenn nur wenig Eigeninitiative von den Kindern erwartet wird und man an der Beobachtung einer konkreten Strategie interessiert ist.

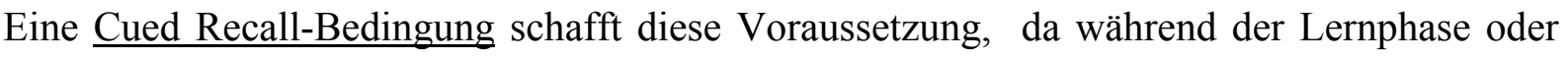
Reproduktionsphase konkrete Hilfestellungen gegeben werden. Diese können beispielsweise 
darin bestehen, dass auf die kategoriale Struktur des Lernmaterials hingewiesen wird (z.B. Ackerman, 1996; Hasselhorn, 1990a, 1990b).

Für die Beobachtung von eigenständigem Strategieeinsatz ist ein anderes experimentelles Design geeigneter. Die Entscheidung hängt davon ab, wie hoch der Schwierigkeitsgrad für die Zielgruppe sein soll (Kapazitätsbeanspruchung).

Bei „Free-Recall“-Aufgaben ist die kognitive Beanspruchung verhältnismäßig hoch, da die Darbietung der Items in einer vorgegebenen Reihenfolge erfolgt. Das Ausmaß der kategorialen Organisation lässt sich daher nur in der Abrufphase erfassen (vgl. Bjorklund, Coyle \& Gaultney, 1992).

Zur Untersuchung individuell kontrollierter Gedächtnisaktivitäten präsentierte Bousfield (1953) seinen Probanden Wort- oder Bildlisten in zufälliger Reihenfolge als Vorbereitung auf eine „Free-Recall“-Anforderung. Die Organisation während der Wiedergabe wurde als Indikator für Vorgänge in der Lernphase genommen. In der darauffolgenden Zeit wurde die begriffliche und wahrnehmungsbezogene Organisation von Lernmaterial von einer Vielzahl von Autoren untersucht (z.B. Bousfield, Esterson et al., 1958). Dabei wurden verschiedene Aspekte näher betrachtet, wie die Bedeutung der Vertrautheit mit den Kategorien (z.B. Bjorklund, Thompson \& Ornstein, 1983; Posnansky, 1978b) und die Anzahl der verwendeten Items und Kategorien (Murphy \& Puff, 1982). Aus dem Befund, dass bei „Free Recall“- Aufgaben mit zunehmendem Alter stärker bei der Wiedergabe geclustert (d.h. nach Gruppen geordnet) wird, wurde geschlussfolgert, dass ältere Kinder ihr Lernverhalten stärker in Vorbereitung auf den Abruf organisieren (vgl. Cole, Frankel \& Sharp, 1971; Moely et al., 1969; Neimark, Slotnick \& Ulrich, 1971).

Bei Sort Recall-Aufgaben haben die Probanden es etwas einfacher, da sie die Lernphase mitgestalten können. Die Instruktion besteht meistens darin, das Lernmaterial (Gegenstände, Wort-; Bild- oder Wort-Bild-Karten) so zu lernen, wie es dem jeweils Lernenden am hilfreichsten erscheint. Oft wird in diesem Zusammenhang auf das Ziel hingewiesen, sich in der anschließenden Reproduktionsphase an möglichst viele Items erinnern zu können (vgl. Coyle \& Bjorklund, 1996, 1997; Sodian \& Schneider, 1999). Auf die kategoriale Struktur des Lernmaterials wird allerdings nicht hingewiesen. Bei diesem Aufgabentyp lässt sich das strategische Organisieren des Lernmaterials bereits in der Lernphase erfassen, da die Kinder die Möglichkeit besitzen, die Lernbedingung mitzugestalten. Dazu gehört auch das Gruppieren des Lernmaterials nach eigenen Kriterien, z.B. nach den implizit enthaltenen Kategorien. In der Abrufphase lässt sich überprüfen, ob zwischen dem in der Lernphase gewählten Ordnungskriterium und dem bei der Wiedergabe 
erkennbaren Ordnungskriterium und/oder der Menge der erinnerten Items ein Zusammenhang besteht.

Bei der Gestaltung einer konkreten Aufgabe zur Erhebung der strategischen Kompetenz von Kindern sind demnach verschiedene externe Faktoren zu berücksichtigen, die einzeln und in Kombination Auswirkungen auf die Leistung der Kinder haben können. Dazu zählen alle Merkmale, die innerhalb der realisierten Aufgabenbedingungen einen Einfluss auf die Bearbeitung der Anforderungen haben können, wie z.B. Instruktion, Lernmaterial, Bearbeitungszeit oder Abrufbedingungen. Diese Faktoren können sowohl beeinflussen, ob eine Strategie überhaupt eingesetzt wird, als auch ob sie effektiv ist (vgl. Hasselhorn, 1986, 1996; Howe \& O’Sullivan, 1990; Schneider \& Pressley, 1989). Dieser Aspekt wird in Abschnitt 2.2.2 näher erörtert.

\subsubsection{Externe Faktoren und ihre Bedeutung für die Strategieanwendung}

Die Bedeutung von externen Faktoren für die Anwendung von Strategien wird daran deutlich, dass sie die Möglichkeit bieten, das Schwierigkeitsniveau der Aufgaben zu verändern und stufenweise $\mathrm{zu}$ variieren. Durch eine entsprechende Manipulation auf Kontextbedingungen können so auch jüngere Kinder in die Lage versetzt werden, strategisches Verhalten $\mathrm{zu}$ zeigen. Darüber hinaus lassen sich so auch Entwicklungsunterschiede beim effizienten Gebrauch der kategorialen Organisationsstrategie aufdecken. Tabelle 2.2 schlüsselt die Variationsmöglichkeiten hinsichtlich des Anspruchsniveaus mit Hilfe der Auswahl spezieller Kontextvariablen (Instruktionsmerkmale, Beschaffenheit des Lernmaterials, Aufgabencharakteristika) noch einmal auf. 
Tabelle 2.2: Experimentelle Erfassung der Strategie des kategorialen Organisierens unter Bezugnahme auf externe Faktoren und die jeweils unterschiedlichen Anspruchsniveaus

\begin{tabular}{|c|c|c|c|c|}
\hline \multirow{2}{*}{\multicolumn{2}{|c|}{ Externe Faktoren }} & \multicolumn{3}{|c|}{ Anspruchsniveau } \\
\hline & & Gering & Mittel & Hoch \\
\hline \multicolumn{2}{|l|}{ Instruktion } & Anleitung / Training & Konkrete Hinweise & $\begin{array}{l}\text { Allgemeine } \\
\text { Lernanforderung }\end{array}$ \\
\hline \multirow{3}{*}{\multicolumn{2}{|c|}{ Lernmaterial }} & Konkrete Objekte & Bilder & Wörter \\
\hline & & $\begin{array}{l}\text { Hohe kategoriale } \\
\text { Typizität }\end{array}$ & $\begin{array}{l}\text { Mittlere kategoriale } \\
\text { Typizität }\end{array}$ & $\begin{array}{l}\text { Niedrige kategoriale } \\
\text { Typizität }\end{array}$ \\
\hline & & Vertraut & Bekannt & Unbekannt \\
\hline \multirow{5}{*}{$\begin{array}{l}\text { Aufgaben- } \\
\text { charakte- } \\
\text { ristika }\end{array}$} & \begin{tabular}{|l} 
Darbietung \\
des Materials
\end{tabular} & $\begin{array}{l}\text { Simultan } \\
\text { (geordnet) }\end{array}$ & $\begin{array}{l}\text { Simultan } \\
\text { (Zufallsanordnung) }\end{array}$ & $\begin{array}{l}\text { Seriell } \\
\text { (in festem Zeittakt) }\end{array}$ \\
\hline & $\begin{array}{l}\text { Enkodier- } \\
\text { phase }\end{array}$ & $\begin{array}{l}\text { Aufforderung zum } \\
\text { Verschieben der Karten } \\
\text { (inkl. Hilfsmittel) }\end{array}$ & $\begin{array}{lr}\text { Erlaubnis } & \text { zum } \\
\text { Verschieben } & \text { der } \\
\text { Karten } & \end{array}$ & $\begin{array}{l}\text { Keine } \\
\text { Manipulations- } \\
\text { möglichkeit }\end{array}$ \\
\hline & & Unbegrenzt Zeit & Ausreichend Zeit & Wenig Zeit \\
\hline & Behaltens- & Kurz & Mittel & Lang \\
\hline & intervall & Ohne Distraktoraufgabe & & $\begin{array}{l}\text { Mit } \\
\text { Distraktoraufgabe }\end{array}$ \\
\hline & $\begin{array}{l}\text { Abruf- } \\
\text { bedingung }\end{array}$ & $\begin{array}{l}\text { Abruf mit kategorialen } \\
\text { Hinweisen }\end{array}$ & Freier Abruf & $\begin{array}{l}\text { Eingeschränkter } \\
\text { Abruf }\end{array}$ \\
\hline
\end{tabular}

\section{Instruktion}

Über den Einsatz spezieller Instruktionen und die verwendeten Formulierungen kann die Nutzung einer Strategie unterstützt werden. Konkret kann die Hilfestellung aus hilfreichen Hinweisen (vgl. Bjorklund et al., 1990; Chi \& Ceci, 1987) oder spezifischen Handlungsanweisungen (Corsale \& Ornstein, 1980; Cox, Ornstein, Naus, Maxfield \& Zimler, 1989) bestehen. Während in „Sort-Recall“"-Bedingungen hilfreiche Hinweise gegeben werden, wird in „,Free-Recall“-Bedingungen häufig bloß die gewünschte Reproduktionsquantität erwähnt (vgl. Bjorklund et al., 1990; Chi \& Ceci, 1987). Die Bandbreite hilfreicher Hinweise reicht von einer unspezifischen Sortierinstruktion (,Du kannst die Karten auch anders hinlegen, wenn Du willst"“) bis hin zur Erwähnung der vorliegenden Gruppierungsmöglichkeit (Benennen der Kategorien). 
Unter Verwendung einer unspezifischen Sortierinstruktion setzten auch 6-Jährige kategoriales Organisieren ein, aber nicht unter einer reinen Spielinstruktion (Schneider \& Sodian, 1991).

Die Bedeutung der verwendeten Formulierungen wird durch die Beobachtung belegt, dass die Drittklässler, die zum Sortieren der Items in zusammengehörige Gruppen instruiert wurden, das stärkste kategoriale Organisieren aufwiesen. Diese Wirkung wurde nicht erzielt, wenn sie aufgefordert wurden, Gruppen zu bilden, die das spätere Reproduzieren erleichtern sollen (Corsale \& Ornstein, 1980), und war ohne Sortieranweisung noch schwächer (Cox, Ornstein, Naus, Maxfild \& Zimler, 1989, Experiment 2).

Durch eine Anleitung zum Sortieren in der Lernphase (z.B. Anweisungen zum Sortieren des Lernmaterials in bedeutungshaltige Gruppen, die mit den zugrundeliegenden Kategorien übereinstimmen) clustern auch jüngere Kinder in der Output-Phase und steigern ihre Reproduktionsleistung (Lange \& Griffith, 1977).

Auch der Zeitpunkt der Gabe der Instruktion spielt eine Rolle. Wenn die Probanden in der Lernphase instruiert werden, beim Lernen das Material in vom Versuchsleiter benannte Kategorien zu sortieren, so führte dieses zu einem hohen Maß kategorialer Zuordnungen bei der Einspeicherung und bei der Wiedergabe der Items. Wurde die Instruktion zur Nutzung der Kategorien aber in der Abrufphase gegeben, so fiel der Effekt wesentlich geringer aus (Kee \& Bell, 1981).

\section{Lernmaterial}

Der Einfluss des Lernmaterials zeigt sich bei der Verwendung unterschiedlicher Itemmodalitäten, d.h. von konkreten Objekten, Bildkärtchen oder Wortmaterial (vgl. Bevan \& Steger, 1971; Cole et al., 1971; Ghatala \& Levin, 1981; Horowitz, 1969). Darüber hinaus lässt sich der Schwierigkeitsgrad durch Variation des Vertrautheitsgrades mit dem Lernmaterial verändern (vgl. Bjorklund \& Buchanan, 1989). Der Zugriff auf die assoziativen Verknüpfungen in der Wissensbasis hängt stark davon ab, wie bekannt die eingesetzten Items sind oder wie eindeutig sie sich bestimmten Kategorien zuordnen lassen (Typizität).

Die Bedeutung des verwendeten Lernmaterials für das kategoriale Organisieren zeigt sich darin, dass bereits Vorschulkinder über eine entsprechende kindgerechte Gestaltung zum Zeigen dieser Strategie veranlasst werden können (vgl. Rossi \& Rossi, 1965; Sodian, Schneider \& Perlmutter, 1986).

Die Itemmodalität scheint das Ausmaß an kategorialer Organisation im Vorschulalter noch nicht zu beeinflussen (vgl. Rossi \& Rossi, 1965). Ab dem Grundschulalter scheint 
kategoriales Organisieren am einfachsten bei der Verwendung konkreter Objekte zu sein, etwas anspruchsvoller ist es im Umgang mit Bildkärtchen (vgl. Bevan \& Steger, 1971). Demgegenüber scheint kategoriales Organisieren bei Wortmaterial einen erhöhten Schwierigkeitsgrad zu besitzen (vgl. Bevan \& Steger, 1971; Cole, Frankel \& Sharp, 1971; Ghatala \& Levin, 1981; Horowitz, 1969).

\section{Aufgabenmerkmale}

Bei den Aufgabenmerkmalen muss zwischen Besonderheiten bei

- den Lernbedingungen,

- $\quad$ dem Behaltensintervall und

- der Abrufsituation

differenziert werden.

Die Anforderungen in der Lernphase werden u.a. durch die Art der Darbietung des Lernmaterials beeinflusst, die seriell (wie bei Free-Recall-Aufgaben) oder simultan (wie bei Sort-Recall-Aufgaben) erfolgen kann. Der entscheidende Faktor dabei ist der verfügbare Freiraum zur selbständigen Verwaltung der Nutzung der eigenen Ressourcen während des Lernprozesses. Eine andere Möglichkeit der Vereinfachung der Lernanforderungen besteht in der bereits nach Kategorien geordneten Vorgabe des Lernmaterials (,category blocked presentation").

Wie entwicklungspsychologische Befunde zeigen, nutzen Zehnjährige ihre Fähigkeit zum strategischen Aktivieren des eigenen kategorialen Wissens nur bei Sort-RecallAnforderungen, da ihnen dort innerhalb des realisierten Aufgabenkontextes ein ausreichender Freiraum zur Verfügung steht. Unterstützende Faktoren sind für Kinder im Alter zwischen 9 und 11 Jahren die Benennung der Items bei der Darbietung (vgl. Furth \& Milgram, 1973; Horowitz, 1969) und das Benennen der Kategorien, die im dargebotenen Lernmaterial vorkommen (vgl. Kobasigawa \& Middleton, 1972, Lange, 1973; Moely, Olson, Halwes \& Flavell, 1969; Shultz, Charness \& Berman, 1973). Während die kategoriale Itempräsentation auch in anderen Altersstufen einen positiven Einfluss auf das kategoriale Organisieren bei der Reproduktion hat (vgl. Arlin \& Brody, 1976), ist dieses beim Benennen der Kategorien nicht der Fall (Lange, 1973; Nelson, 1969; Williams \& Goulet, 1975).

\section{Bedingungen in der Enkodierphase}

Da Lern- und Erinnerungsprozesse eng miteinander zusammenhängen, ist es schwer, zwischen der Qualität der Enkodier- und der Abrufprozesse zu unterscheiden. Um 
systematische Unterschiede in den Lernstrategien zu minimieren, wurde in einigen Studien in der Lernphase von den Probanden verlangt, die Items genau nach ihren taxonomischen Gruppen zu sortieren (Ceci \& Howe, 1978; Ceci, Lea \& Howe, 1980; Williams \& Goulet, 1975; Worden, 1974).

In Studien, in denen das Organisationsverhalten hauptsächlich in der Lernphase (d.h. bei der Einspeicherung von Informationen) untersucht wurde, wurde ein wesentlich stärkerer Strategiegebrauch vermutet als wenn das Verhalten vor allem in der Abrufphase untersucht wurde (vgl. Kee \& Bell, 1981; Ornstein, Baker-Ward \& Naus, 1988; Schneider, 1986). Forscher, die sich mit der Einspeicherung beschäftigen, nehmen an, dass bewusste Gedächtnisprozesse während der Enkodierung ablaufen, wenn spontanes Sortieren oder andere effiziente Lernprozesse beim Lernen beobachtbar sind. Schneider (1986) untersuchte das Sortierverhalten und andere Lernmöglichkeiten bei Zweit- und Viertklässlern. Kinder, die sortierten, zeigten auch andere strategische Aktivitäten, z.B. Rehearsal und Selbstüberprüfung (self-testing).

\section{Bedingungen in der Abrufphase}

Ein Großteil dessen, was sich im Gebiet des Gedächtnisabrufs entwickelt, besteht aus der Möglichkeit der intelligenten, d.h. systematischen, flexiblen, erschöpfenden und selektiven Durchsuchung des Langzeitgedächtnisses. Soll untersucht werden, in welcher Reihenfolge Lerninhalte (unabhängig von der Darbietungsreihenfolge) abgerufen werden, ist es notwendig, die Enkodier-Variabilität zu erhöhen. Dafür bietet sich ein Design an, in dem die zu lernenden Listen mehrere Male hintereinander in unterschiedlicher, jeweils zufälliger Reihenfolge dargeboten werden. Dadurch kommen die Probanden mit jedem Item in einem unterschiedlichen Lernkontext in Berührung und müssen den anschließenden Abrufkontext individuell festlegen. Die Reihenfolge, in der das Item mit anderen Items abgerufen wird, unterscheidet sich dadurch meistens von der Reihenfolge beim Lernen (Waters \& McAlaster, 1983; Waters \& Waters, 1976, 1979).

Die strategische Kompetenz in der Abrufphase lässt sich auch überprüfen, indem ein Design gewählt wird, welches unabhängig von der Gedächtnisleistung die Anwendung einer speziellen (optimalen) Abrufstrategie erfordert. Salatas und Flavell (1976b) ließen ihre Probanden Items bis zu einem hohen Leistungsniveau lernen, d.h. bis sie alle Items der Liste perfekt unter der eingeschränkten Wiedergabebedingung wiedergeben konnten (verfügbare Kategorie-Hinweise, aber vollständige Wiedergabe der erforderlichen Kategoriemitglieder). 
Anschließend wurden indirekte Abruffragen gestellt, die eine Durchsuchung der Items unter Nutzung anderer Dimensionen als der kategorialen, die während der Lernphase angegeben worden war, forderten. Die günstigste Vorgehensweise bestand in der Wiedergabe der Kategorienamen, die während der Lernphase benutzt worden waren, der anschließenden erschöpfende Suche in jeder dieser Kategorien und der Beurteilung von jedem Item in jeder Kategorie daraufhin, ob es die in der Frage angeführten Anforderungen erfüllt.

Will man einfachere Bedingungen schaffen, so kann man auch in der Abrufphase Hilfen in Form von Hinweisreizen zur Verfügung stellen. Als Hinweisreize können beispielsweise Bildkarten eingesetzt werden, die die Kategorien repräsentierten (Kobasigawa, 1974).

Im Allgemeinen entwickelt sich die Fähigkeit, Informationen effektiv aus dem Langzeitgedächtnis abzurufen, relativ spät in der Kindheit, besonders wenn der Gebrauch von internalen Gedächtnishinweisen benötigt wird. Dass Enkodier- und Abrufprozesse nur schwerlich unabhängig voneinander untersucht werden können, macht es schwierig, den Gedächtnisanteil zu bestimmen, aufgrund dessen der Einsatz von Enkodier- verglichen mit Abruf-Heuristiken erfolgt.

Mögliche Defizite beim Abruf können darin bestehen, dass 1.) Kinder nicht wahrnehmen, dass internale oder externale Gedächtnis-Hinweise mögliche Gedächtnishilfen sind, 2.) den Kindern Strategien fehlen, um die Zielinformation im Gedächtnis aufzufinden oder 3.) die Kinder nicht genügend Erfahrung mit dem Problem haben, um zu beurteilen, wann der Suchprozess als abgeschlossen angesehen werden kann (Kobasigawa, 1977).

Kobasigawa (1974) fand in seinen Untersuchungen, dass Sechsjährige die zur Verfügung stehenden Abrufhinweise (Bildkarten für die Kategorien) in der Testphase meistens übersahen. Acht- und Elfjährige nutzten diese Hinweise systematischer.

Diese alterskorrelierten Unterschiede können aber auch dadurch zustande kommen, dass die älteren Kinder sich durch die Organisation des Lernmaterials in Kategorien während der Lernphase einfach bessere Bedingungen für den Abruf geschaffen haben. Eine große Variabilität beim Lernen, wie sie bei der wiederholten Itemdarbietung in randomisierter Reihenfolge erreicht wird, beeinflusst jüngere Kinder stärker negativ als ältere Kinder, so dass diese Experimente schlechtere Bedingungen für das effektive Lernen von jüngeren Kindern liefern als für ältere Kinder (Kobasigawa, 1974; Lange, 1973; Scribner \& Cole, 1972).

Aus den beobachteten Altersdifferenzen wurde von verschiedenen Autoren auf das Vorliegen systematischer Entwicklungsunterschiede beim effizienten Gebrauch von Abrufstrategien geschlossen. Die Fähigkeit älterer Kinder, auch unter Bedingungen mit 
hohem Schwierigkeitsniveau, wie in Free-Recall-Aufgaben, gute Leistungen zu erbringen, wurde dabei vor allem auf die größere Flexibilität ihrer Abrufstrategien zurückgeführt (Ceci \& Howe, 1978; Hasher \& Clifton, 1974).

In Abhängigkeit vom Schwierigkeitsniveau der Anforderungen werden in verschiedenen Altersstufen Abrufstrategien mit unterschiedlichem Komplexitätsgrad eingesetzt. Einfache Abrufstrategien (z.B. der Gebrauch von externalen AssoziationsHinweisen) können auch von Kindern im Vorschul- und Grundschulalter genutzt werden (Wellman, 1985b). Sehr komplexe Abrufstrategien gelangen hingegen oft erst nach der Grundschulzeit in das strategische Repertoir der Kinder. Wesentliche Elemente dabei sind die Reorganisation von gespeicherter Information in Kombination mit einer erschöpfenden Suche nach relevanten Gedächtnisinhalten und die anschließende sorgfältige Auswertung der Ergebnisse dieser Suche. Bezogen auf die Strategie des kategorialen Organisierens würde dieses beispielsweise bedeuten, dass zuerst die in der Lernphase benutzten Kategorienamen wiedergegeben werden, dann die Kategorien erschöpfend durchsucht werden und anschließend jedes Item auf seine Relevanz hin untersucht wird. In der Untersuchung von Salatas und Flavell (1976b) zeigte sich, dass sich zwar die meisten Studenten für diese optimale Vorgehensweise entschieden, aber nur wenige der 6- und 9jährigen Schulkinder.

\subsubsection{Experimentelle Designs}

In Abhängigkeit von der jeweiligen Fragestellung kann es für die Identifizierung nutzungsdefizitärer Kinder unterschiedliche Kriterien geben, die mit unterschiedlichen experimentellen Herangehensweisen überprüft werden können (vgl. Tabelle 2.3). Gesucht wird jeweils nach direkten oder zumindest korrelativen Zusammenhängen zwischen Strategieanwendung und Reproduktionsleistung. 
Tabelle 2.3: Empirische Überprüfungen der Strategieproduktion und -effektivität

\begin{tabular}{|l|l|l|}
\hline \multicolumn{2}{|l|}{ Art der Fragestellung } & Überprüfung über \\
\hline Kompetenzunterschiede & Querschnittstudie \\
\hline Kompetenzveränderungen & kurzfristig & Mikrogenetische Studie \\
\cline { 2 - 3 } & langfristig & Längsschnittstudie \\
\hline Potentielle Kompetenzen & Trainingsstudie \\
\hline Informationseinspeicherung & Lernphase \\
\hline Informationsabruf & Abrufphase \\
\hline erste Nutzung kategorialer Strukturen & Selektive Gedächtnisanforderung \\
\hline elaborierte Nutzung kategorialer Strukturen & Kategoriale Organisationsanforderung \\
\hline
\end{tabular}

Das Auftreten eines Nutzungsdefizits lässt sich auf vielfältige Art und Weise mit unterschiedlichen experimentellen Designs (z.B. Querschnittstudien, Längsschnittstudien, mikrogenetische Studien, Trainingsstudien) untersuchen (vgl. Lockhart, 2000).

\section{Querschnittstudien}

In Querschnittstudien besteht die Möglichkeit, die Effektivität des Strategiegebrauchs bei verschiedenen Gruppen $\mathrm{zu}$ untersuchen, die sich nach unterschiedlichen Kriterien zusammenstellen lassen (z.B. Alter, Verhalten bei Aufgabenbearbeitung).

Auf Gruppenebene sollte sich ein Nutzungsdefizit beim Vergleich verschiedener Altersgruppen darin zeigen, dass ältere Kinder eine höhere Reproduktionsleistung zeigen als jüngere Kinder, die genau so strategisch handeln.

Problematisch an dieser Form der Überprüfung der strategischen Kompetenz ist, dass Altersunterschiede in der Abrufleistung gewöhnlich auch mit Altersunterschieden in anderen (nicht-strategischen) Prozessen einhergehen (Informationsverarbeitungsgeschwindigkeit, funktionale Arbeitsgedächtniskapazität, bereichsspezifisches und Weltwissen sowie metamemoriale Kompetenz). Diese Konfundierung lässt keine eindeutigen Schlussfolgerungen darüber $\mathrm{zu}$, welchen Anteil das strategische Verhalten an der Leistungssteigerung hat (vgl. Schlagmüller \& Schneider, 2002).

Die Analyse kann in derselben oder in verschiedenen Altersgruppen durchgeführt werden. In derselben Altersgruppe sprechen gleiche Reproduktionsleistungen von strategischen und nicht-strategischen Kindern oder schlechtere Reproduktionsleistungen der strategischen Kinder für ein Nutzungsdefizit. Auch die Korrelation zwischen strategischem 
Verhalten und Abrufleistung kann als Indikator dafür herangezogen werden, dass die Abrufleistung durch die Strategieanwendung gesteuert wird.

In unterschiedlichen Altersgruppen kommt dem Auftreten eines Nutzungsdefizits eine andere Bedeutung zu. Während ein Nutzungsdefizit bei Viertklässlern ein Indikator für eine relative Entwicklungsverzögerung ist, da die meisten Kinder dieser Altersstufe bereits effektiv Strategien anwenden, kann ein Nutzungsdefizit bei jüngeren Kindern (Zweit- und Drittklässlern) als Entwicklungsvorsprung interpretiert werden. Diese Annahme wird durch die jeweiligen Reproduktionsleistungen unterstützt (Coyle \& Bjorklund, 1996). Das Leistungsniveau von quasi-nutzungsdefizitären Kindern unter den Viertklässlern ähnelt dem der strategischen nicht-nutzungsdefizitären Kinder stärker als dem der nutzungsdefizitären Kinder (Bjorklund \& Coyle, 1995).

\section{Längsschnitt- und Mikrogenetische Studien}

Längsschnittstudien sind besonders geeignet, um Aussagen über individuelle Entwicklungsverläufe zu treffen und sind aus diesem Grund besonders wichtig (Ornstein \& Haden, 2001). Auf Individualebene würde sich ein Nutzungsdefizit darin äußern, dass ein im Laufe der Durchgänge zunehmender Strategieeinsatz nicht von einer entsprechenden Leistungsverbesserung begleitet wird. Dabei kann auch die möglicherweise zunehmende Effektivität der Strategieanwendung berücksichtigt werden, die evtl. zu Beginn der Untersuchung noch nutzungsdefizitär war. Ein anderer Hinweis auf ein Nutzungsdefizit wäre die ausbleibende Verbesserung bzw. sogar Verschlechterung der Wiedergabeleistung unter dem Einsatz einer Strategie gegenüber dem Ausbleiben derselben. Darüber hinaus spricht auch eine abnehmende Reproduktionsleistung beim Einsatz von mehreren Strategien für ein Nutzungsdefizit.

Ein charakteristisches Beispiel für die längsschnittliche Untersuchung der Strategieentwicklung stellt die LOGIK-Studie dar (vgl. Weber \& Stefanek, 1998). Sodian und Schneider (1999) untersuchten im Rahmen dieses Projektes die Entwicklung der Strategie des kategorialen Organisierens mit einer „Sort-Recall“-Anforderung. Dabei bekamen Kinder vom 4. bis zum 12. Lebensjahr im zweijährlichen Abstand die Aufgabe, sich kategorisierbare Items (Spielzeug für Vier- und Sechsjährige; Bildkarten für die älteren Kinder) einzuprägen, wobei das Lernmaterial analog zu anderen Untersuchungen (z.B. Coyle \& Bjorklund, 1996) in der Lernphase beliebig verschoben werden durfte. 
Mikrogenetische Studien bieten die Möglichkeit, Entwicklungsveränderungen zu entdecken, die in sehr kurzen Phasen ablaufen (vgl. Miller \& Coyle, 1999). Gleichzeitig wirken allerdings in besonderem Maße Übungs- und Ermüdungseffekte. Ein typisches Beispiel für ein mikrogenetisches Design zur Untersuchung der kategorialen Organisationsstrategie stellt die Studie von Schlagmüller und Schneider (2002) dar. In einem Zeitraum von 12 Wochen erhielten Kinder der dritten und vierten Klasse an neun Testterminen eine semantische Kategorisierungsaufgabe. Dabei sollten 20 nach Oberbegriffen kategorisierbare Wortbildkärtchen gelernt und später wiedergegeben werden. Den Kindern wurde in der Lernphase erlaubt, das Lernmaterial in beliebiger Anordnung vor sich hinzulegen, d.h. sie hatten die Möglichkeit, nach Kategorien zu sortieren, wurden aber nicht explizit dazu aufgefordert.

Andere Autoren entschieden sich für noch kürzere Untersuchungsabstände (z.B. Bjorklund, Coyle \& Gaultney, 1992; Coyle \& Bjorklund, 1996, 1997). In diesen Studien wurden die Aufgaben innerhalb einer Sitzung mehrfach dargeboten. Bjorklund et al. (1992) untersuchten Kindergartenkinder, Drittklässler und Achtklässler mit „Free-Recall“Anforderungen, bei denen kategorisierbare Wörter in ungeordneter Reihenfolge vorgelesen wurden. Coyle und Bjorklund (1996, 1997) arbeiteten mit einem „Sort Recall“-Design, indem sie ihren Probanden (Zweit-, Dritt- und Viertklässler) Wortkärtchen darboten und Manipulationen am Lernmaterial in der Lernphase (z.B. Gruppieren) ausdrücklich zuließen.

\section{Trainingsstudien}

In Trainingsstudien könnte sich ein Nutzungsdefizit darin zeigen, dass Kinder eine Strategie zwar lernen und auch einsetzen, aber in der Aufgabe entweder in Bezug auf eine frühere Baseline oder in Bezug auf eine Kontrollgruppe mit nicht-trainierten Kindern keinen Leistungsvorteil haben. Auf der anderen Seite könnte sich das Nutzungsdefizit auch darin äußern, dass das Training bei jüngeren und älteren Kindern zu einem vergleichbaren Strategiegebrauch führt, aber bei den älteren Kindern eine deutlichere Leistungsverbesserung bewirkt. Das entscheidende Kriterium für die Identifikation eines Nutzungsdefizits ist das Ausmaß an Eigenständigkeit bzw. Spontanität bei der Strategieproduktion. Die Kinder sollten die Strategie selbständig einsetzen, aber keine Leistungsverbesserungen in Form einer erhöhten Wiedergabeleistung erzielen.

Nach der Betrachtung der Effektivität von Strategien im Hinblick auf verschiedene experimentelle Designs soll nun das Augenmerk auf empirische Befunde zur effektiven 
Nutzung kategorialer Strategien in Abhängigkeit von verschiedenen Aufgabentypen gerichtet werden. Obwohl das Phänomen des Nutzungsdefizits lange Zeit in der Forschung nicht ausdrücklich thematisiert wurde, konnten Miller und Seier (1994) bei einer Vielzahl von Forschungsarbeiten (41 von 45 Studien von 1966 bis Mitte 1992) Hinweise für die Existenz des Nutzungsdefizits bei unterschiedlichen Strategien (Sortieren, Clustern, Rehearsal, Elaboration, Nutzung von Abrufhinweisen, etc.) finden. Die Autoren konzentrierten sich dabei auf Untersuchungen, in denen ein selbständiger Strategiegebrauch eindeutig identifiziert werden konnte. Der Zeitpunkt des Auftretens des Nutzungsdefizits variierte in Abhängigkeit von der jeweiligen Aufgabe mit dem Alter. Die meisten Belege betrafen die postulierten Altersunterschiede, d.h. die Überlegenheit von älteren Strategieentdeckern gegenüber jüngeren und Unterschiede in den Zusammenhängen zwischen dem strategischen Verhalten und der Reproduktionsleistung. Demgegenüber standen allerdings auch Studien, die die beschriebenen Phänomene weniger eindeutig oder gar nicht zeigten. In einigen Untersuchungen verfügten die jungen strategischen Kinder über eine bessere Wiedergabeleistung als gleichaltrige nicht-strategische Kinder (z.B. Flavell, Beach \& Chinsky, 1966; Schneider, 1986), in anderen Studien gab es unter den Strategen keine systematischen Unterschiede, weder hinsichtlich des Alters (z.B. Hasselhorn, 1990, Experiment 1; Weissberg \& Paris, 1986) noch in Bezug auf die Korrelationen zwischen strategischem Verhalten und Wiedergabe (z.B. Andreassen \& Waters, 1989).

Um die Nützlichkeit einer bestimmten Verhaltensweise beurteilen zu können, muss allerdings die Art und Qualität des Verhaltens im Zusammenhang mit der jeweiligen Entwicklungphase gesehen werden. Wenn man von kategorialem Organisieren spricht, so versteht man darunter meistens die Gruppierung von Lernmaterial, das aus einer Vielzahl von Kategorien besteht. Solche kategorialen Organisationsstrategien werden am Ende der Grundschulzeit von den meisten Kindern in unterschiedlichen Bedingungen spontan und effizient genutzt (Hasselhorn, 1992; Schneider, 1986). Daran wird bereits deutlich, dass dieses Verhalten, auf das an späterer Stelle noch näher eingegangen wird, sich bereits auf einem relativ hohen Niveau befindet. Die Kinder, die dieses Verhalten zeigen befinden sich schon in einer relativ späten Entwicklungsphase. Der erste Umgang mit kategorialen Strukturen kann aber auch schon bei jungen Kindern untersucht werden, indem ihnen weniger Kategorien vorgegeben werden. Bereits durch die Konfrontation mit zwei unterschiedlichen Kategorien lässt sich bei jüngeren Kindern erkennen, ob eine gezielte Differenzierung innerhalb des Lernmaterials gelingt. Um bereits erste Ansätze strategischer Kompetenzen aufzuzeigen soll auch auf den Einsatz einer solchen selektiven Gedächtnisstrategie 
eingegangen werden. Dabei wird die Lernanforderung nicht planlos, sondern zielgerichtet unter Fokussierung auf wesentliche Gesichtspunkte bearbeitet, indem relevantes von irrelevantem Lernmaterial getrennt wird (Miller \& Seier, 1994). Wie bereits angemerkt, sind für die wirkliche Nutzung kategorialer Strukturen allerdings experimentelle Designs erforderlich, die das Erkennen und die Handhabung von komplexen Ordnungsstrukturen und hierarchischen Beziehungen erfordern (z.B. Bjorklund et al., 1992; Hasselhorn, 1996; Sodian \& Schneider, 1999).

\section{Frühe Nutzung kategorialer Strukturen am Beispiel der selektiven Aufmerksam- keit als Strategie}

Miller und ihre Kollegen (z.B. Miller, 1994; Miller \& Aloise-Young, 1995; Miller \& Seier, 1994) setzten zur Untersuchung des Nutzungsdefizit-Phänomens eine Aufgabe zur selektiven Aufmerksamkeit ein. Bei diesem Aufgabentyp besteht das Lernmaterial aus Exemplaren von unterschiedlichen Kategorien (z.B. Tiere und Haushaltsgegenstände), den Kindern wird aber gesagt, dass sie sich nur merken sollen, wo die Exemplare der einen Kategorie (z.B. Tiere) sind. Die zu lernenden Objekte sind hinter Türen versteckt, die aber während der Lernphase von dem Kind beliebig nacheinander geöffnet werden können. Zeichnung auf der Oberfläche der Türen liefern Hinweise darauf, was sich darunter befindet (z.B. ein Haus für Haushaltsgegenstände). Sehr junge Kinder (Dreijährige) wenden bei dieser Anforderung eine räumliche Strategie an und öffnen nacheinander alle Türen. Im Alter von 10 Jahren erkennen die meisten Kinder, dass es effizienter ist, nur die relevanten Türen, d.h. diejenigen, die die $\mathrm{zu}$ erinnernden Objekte enthalten, $\mathrm{zu}$ öffnen und verhalten sich angemessen strategisch (Miller et al., 1986). Bei jüngeren Kindern (5-9 Jahre) kann eine selektive Aufmerksamkeitsstrategie durch das Hinzufügen einer unterstützenden Geschichte hervorgerufen werden (z.B. Woody-Ramsey \& Miller, 1988).

Die Beobachtungen lassen sich, wie bereits in Kapitel 2.1.3 ausgeführt, nach Miller und Seier (1994) verschiedenen Klassen von Hinweisen für das Auftauchen eines Nutzungsdefizits zuordnen:

\section{Hinweise auf Altersdifferenzen:}

Altersunterschiede können sich darin äußern, dass die korrelativen Zusammenhänge zwischen der spontanen Strategieproduktion und der Wiedergabeleistung bei jüngeren Kindern niedriger sind als bei älteren (Miller et al., 1986; Miller, Seier, Probert \& Aloise, 1991; Miller, Woody-Ramsey \& Aloise, 1991; Woody-Ramsey \& Miller, 
1988). Einen anderen Hinweis erhält man, wenn unter den Strategen die älteren Kinder deutlich mehr erinnern können als die jüngeren Kinder (DeMarie-Dreblow \& Miller, 1988; Miller et al., 1986; Miller, Seier, Probert \& Aloise, 1991; Miller et al., 1991). Darüber hinaus sollte der durch den Strategieeinsatz erzielte Leistungsvorteil im Vergleich zum Nicht-Einsatz der Strategie bei älteren Kindern wesentlich stärker ausgeprägt sein als bei jüngeren Kindern (Miller et al., 1986).

\section{Hinweise auf Defizite beim ersten Strategieeinsatz:}

Ein deutlicher Beleg für Defizite beim ersten Strategieeinsatz besteht darin, dass junge Strategen keine bessere Erinnerungsleistung haben als gleichaltrige Nicht-Strategen (Miller et al., 1991; Woody-Ramsey \& Miller, 1988). Eine niedrige Reproduktionsleistung trotz eines hohen Niveaus an spontaner Strategieproduktion (Woody-Ramsey \& Miller, 1988; Miller et al., 1994; Miller et al., 1991) oder die im Vergleich zum unstrategischen Vorgehen schwächere Leistung unter spontaner Strategieproduktion (DeMarie-Dreblow \& Miller, 1988; Newman, 1990; Bjorkund et al., 1992) liefern weitere Hinweise.

3. Hinweise für Defizite in der Anfangsphase des Strategiegebrauchs:

Die dritte Klasse von Hinweisen bezieht sich auf die Anfangsphase der Strategieproduktion, in der der reale Nutzen des Verhaltens noch hinter dem potentiell erreichbaren zurückbleibt. Diese relative Ineffektivität der Strategie kann sich darin äußern, dass der korrelative Zusammenhang zwischen spontaner Strategieproduktion und Wiedergabeleistung im Laufe der Zeit signifikant wird (DeMarie-Dreblow \& Miller, 1988). Ein weiterer Beleg ist darin zu sehen, dass Kinder, die in nur in einigen Durchgängen strategisch sind, in den Durchgängen, in denen sie strategisch sind, nicht bedeutsam mehr reproduzieren als in den Durchgängen, in denen sie es nicht sind (Bjorklund et al., 1992). Darüber hinaus sollte $\mathrm{zu}$ beobachten sein, dass die Anwendung eine Strategie in verschiedenen Durchgängen bei den Kindern am Anfang noch keine Steigerung der Wiedergabeleistung bewirkt, der Erfolg sich aber einstellt, wenn die Anwendung der Strategie fortgesetzt wird (Bjorklund et al., 1992).

Obwohl in der Literatur die meisten empirischen Belege für das Vorliegen eines Nutzungsdefizits Altersdifferenzen behandeln, ist für Miller und Seier (1994) der direkteste und zwingendste Beweis für das Nutzungsdefizit die zunehmende Verbesserung des 
strategischen Verhaltens der Kinder im Laufe der Durchgänge ohne eine korrespondierende Zunahme der Wiedergabeleistung. Um bei Kindern den Übergang von einem uneffektiven zu einem leistungsdienlichen Strategiegebrauch $\mathrm{zu}$ analysieren, sollten daher möglichst alle Phasen im Entwicklungsverlauf verfolgt werden, d.h. von der erstmaligen Strategieproduktion über die Anfangsphase, in der die Strategie nützlich wird, bis sie mit zunehmendem Alter oder Erfahrung ihr individuelles Optimum erreichen. Dieses erfordert die Analyse des Verlaufs der Strategieproduktion und der damit einhergehenden Effektivität von einzelnen Kindern über mehrere Durchgänge. Auf diese Art lassen sich auch sehr kurze Phasen im Entwicklungsverlauf (z.B. die Abnahme der Reproduktionsleistung unmittelbar nach der ersten Strategieproduktion) identifizieren.

\section{Späte Nutzung kategorialer Strukturen am Beispiel der kategorialen Organisationsstrategie}

Bei älteren Kindern ist die Fähigkeit zur Nutzung kategorialer Strukturen auch direkt messbar, indem Aufgaben dargeboten werden, in denen eine kategoriale Organisationsstrategie forciert wird. Zusammen mit verschiedenen Kollegen beschäftigte sich Bjorklund mit dem Phänomen des Nutzungsdefizits bei „Free-Recall“-Aufgaben (Bjorklund et al., 1992). Bei diesen Untersuchungen ließen sich die wiederzugebenden Items eindeutig Kategorien zuordnen (z.B. Fahrzeuge), so dass sie von den Kindern für eine Ordnungsstrategie herangezogen werden konnten. Während der Lernphase bestand die Möglichkeit, das Lernmaterial zu sortieren, in der Abrufphase konnten die Items entsprechend dieser Gruppierungen (in Clustern) reproduziert werden. Auf die bestehende Diskussion, ob das Clustern eine Organisationsstrategie darstellt (z.B. Ornstein, Baker-Ward \& Naus, 1988) oder nur eine semantische Assoziation zwischen den Items widerspiegelt, die automatisch zum Clustern führt (z.B. Bjorklund \& Jacobs, 1985), wird in Kapitel 2.2.4. noch eingegangen.

Schon bevor Bjorklund sich direkt mit dem Phänomen des Nutzungsdefizits beschäftigte berichtete er in früheren Untersuchungen von einer geringeren Strategieeffektivität, die sich als Nutzungsdefizit interpretieren lässt. Diese Leistungseinbußen konnten meistens für jüngere Kinder im Vergleich $\mathrm{zu}$ älteren gefunden werden. Dieses entspricht dem ersten Nutzungsdefizitbeweis von Miller und Seier (1994), dem Vorliegen von Altersdifferenzen. 
Bjorklund und Zeman (1982, Experiment 2) beobachteten, dass Elfjährige den jüngeren Kindern (Neunjährige) nur in ihrer Clusterleistung überlegen waren, aber nicht in der Reproduktion. Wenn Neun- und Dreizehnjährige im gleichen Ausmaß clusterten, dann konnten ältere Kinder mehr Items wiedergeben als jüngere (Bjorklund \& Harnishfeger, 1987; Studie 2). Bjorklund und Bjorklund (1985) fanden dieses Muster auch schon bei Sechs- und Achtjährigen. Trotz vergleichbarer Cluster-Niveaus bei einer „Free-Recall“-Aufgabe sind die älteren Kinder den jüngeren überlegen. Höhere Korrelation zwischen Clustern und Wiedergabe bei den älteren strategischen Kindern als bei den jüngeren strategischen Kindern fanden Bjorklund und Harnishfeger (1987; Studie 2) und Bjorklund und Jacobs (1985).

Zur direkten Untersuchung der Existenz des Nutzungsdefizits führten Bjorklund et al. (1992) mit Kindern im Alter von 5, 8 und 13 Jahren „Free-Recall“-Aufgaben durch, in denen fünf Durchgänge mit unterschiedlichen Listen und Kategorien präsentiert wurden. Obwohl sowohl Acht- als auch Dreizehnjährige spontan clusterten, korrelierte das Clustern nur bei den älteren Kindern mit der Wiedergabe. Ein Vergleich der Durchgänge, in denen eine vergleichbare Clusterleistung vorlag, ergab eine höhere Wiedergabeleistung der 13jährigen gegenüber den jüngeren Kindern. Diese Befunde unterstützen die Muster, die mit der selektiven Aufmerksamkeitsstrategie gefunden wurden.

Für den von Miller und Seier (1994) aufgestellten dritten Nutzungsdefizitbeweis, die Veränderungen der Effektivität über mehrere Durchgänge, gab es in der Studie von Bjorklund und Bjorklund (1985) einen ersten Hinweis. Dort führte die Aufforderung, die Mitschüler nach ihrem Geschlecht oder ihrer Sitzordnung wiederzugeben, nur zu einer Verbesserung der Clusterleistung, steigerte aber nicht die Reproduktionsleistung.

Für eine direkte Untersuchung dieses Phänomens sollten die Effektivität des Strategieeinsatzes bei Kindern untersucht werden, die eine Strategie spontan während der Experimental-Situation erwarben. Bjorklund und seine Kollegen analysierten die korrelativen Veränderungen zwischen Clustern und Wiedergabe im Verlauf der Durchgänge (Bjorklund et al., 1992). Während das Clustern bei den Neunjährigen im Laufe der Zeit deutlich zunahm, stagnierte ihre Wiedergabeleistung bzw. ging sogar leicht zurück. Bei den 13jährigen war hingegen eine parallele Verbesserung der Clusterleistung und der Wiedergabe zu verzeichnen. Der Zeitpunkt des größten Clusterzuwachses fiel mit der höchsten Reproduktionssteigerung zusammen.

Aufgrund der Tatsache, dass eine zunehmende Clusterleistung nicht von einer zunehmenden Wiedergabe begleitet wurde, wurden $38 \%$ der Neunjährigen als 
nutzungsdefizient klassifiziert. Viele dieser Kinder profitierten zu einem späteren Zeitpunkt von ihrem Strategiegebrauch.

Die Clusterleistung lässt sich in diesen Untersuchungen eher auf strategische als auf automatische Prozesse zurückführen, da Bjorklund und seine Kollegen kategoriale Items ohne hohe Assoziationen zwischen den Wörtern auswählten. Untermauert wird dieser Hinweis durch eine Studie von Bjorklund, Schneider, Cassel und Ashley (1994), die mit einer Organisationsstrategie beim Gruppieren von Items in Kategorien während einer Lernphase vor dem Abruf zu ähnlichen Ergebnissen kamen. Neun- und Zehnjährige mit hohen und niedrigen Intelligenzwerten erhielten ein Strategietraining und wurden später mit vergleichbaren Anforderungen konfrontiert, ohne erneut zur Anwendung der geübten Strategie aufgefordert zu werden. Sowohl in einem folgenden Durchgang als auch eine Woche später war bei den meisten Kindern ein hohes Ausmaß an spontanem Sortieren und/oder Clustern zu verzeichnen. Allerdings sank die Wiedergabeleistung bei nahezu der Hälfte aller Kinder in diesen Durchgängen auf das Niveau in den Baseline-Durchgängen, sogar bei den perfekten Strategen. Darüber hinaus waren in der Gruppe der vollständigen Strategen die Zehnjährigen den Neunjährigen in ihrer Wiedergabe überlegen und ebenso wie die Kinder mit einem hohen Intelligenzquotient (IQ) denen mit einem niedrigen IQ-Wert. Für die Clusterleistung fand sich ein ähnliches, wenn auch schwächer ausgeprägtes Muster.

\section{Besonderheiten unter Berücksichtigung des experimentellen Designs}

Auch unter Verwendung unterschiedlicher experimenteller Designs gab es kein einheitliches Bild hinsichtlich des Auftretens des Nutzungsdefizits, sondern sowohl Belege für ineffektiven als auch für effektiven Strategiegebrauch.

Bjorklund und seine Kollegen (Bjorklund \& Coyle, 1995; Bjorklund, Coyle \& Gaultney, 1992; Bjorklund, Schneider, Cassel \& Ashley, 1994) vertraten die Ansicht, dass es beim Lernen kategorisierbarer Listen Hinweise auf das Vorliegen des Nutzungsdefizits gibt. Das Phänomen konnte von Bjorklund und seinen Kollegen (1992) in der Abrufphase beobachtet werden, in der eine Verbesserung der Clusterleistung nicht mit einer steigenden Wiedergabeleistung einherging. Es zeigte sich aber darüber hinaus auch in der Lernphase in Form eines wenig leistungsdienlichen Sortierverhaltens (Bjorklund, Schneider, Cassel \& Ashley, 1994). Übungsbedingt verbesserten sich sowohl der Strategiegebrauch in der Lernund Abrufphase als auch die Wiedergabeleistung. Während das Niveau des strategischen Verhaltens (Sortieren und Clustern) allerdings auf einem hohen Niveau gehalten werden 
konnte, sank die Reproduktionsleistung später wieder ab. Unter den Strategen erzielten die älteren Kinder (Viertklässler) im Vergleich zu den Drittklässlern besonders hohe Wiedergabeleistungen. Von einem Nutzungsdefizit in den individuellen Ergebnismustern wurde ausgegangen, wenn die Sortier- und/oder Clusterleistung im 3. und 4. Durchgang größer war als im ersten Durchgang, aber die Wiedergabe sich nicht um mindestens 2 Wörter gesteigert hatte. Unter diesem Kriterium erwies sich ein Drittel der Kinder als nutzungsdefizitär. Etwas höher war der Anteil der Kinder, die ein Nutzungsdefizit nur für die Sortierleistung (37\% bzw. 49\%) oder das Clustern (30\% bzw. 33\%) aufwiesen. Das Nutzungsdefizit scheint demnach kein altersbezogenes Stadium in der Entwicklung, sondern eine Phase im Erwerb einer Teil-Strategie in einem speziellen Kontext zu sein.

Hinweise auf einen ineffektiven Strategieeinsatz wurden auch in Trainingsstudien gefunden, wenn die Strategie im Vorfeld geübt wurde. Dort wurde der Nutzen der eigenständigen Strategieproduktion zu einem späteren Zeitpunkt betrachtet. Die Effektivität einer Strategie war meistens dann besonders gering, wenn sie von den Kindern selbständig eingesetzt werden musste (Bjorklund, Miller, Coyle \& Slawinski, 1997). Beim Auftreten von Nutzungsdefiziten in Trainingsstudien war ein eindeutiger Alterstrend beobachtbar. Während der Anteil an nutzungsdefizitären Kindern bei den Siebenjährigen noch bei 66\% lag, sank er bei den Acht- und Zehnjährigen auf $42 \%$ und lag bei den Elfjährigen nur noch bei 37\% (Bjorklund et al., 1997). Bjorklund und Harnishfeger (1987) konnten beobachten, dass trainierte Drittklässler zwar ihren Strategiegebrauch steigerten, nicht aber ihre Gedächtnisleistung. Verschiedene andere Studien zeigten allerdings, dass trainierte ältere Kinder stärker von einem Training profitieren als jüngere Kinder (Bjorklund \& Buchanan, 1989; Lange, Guttentag \& Nida, 1990; Lange \& Pierce, 1992).

Hinweise für die Existenz des Nutzungsdefizits gab es auch in mikrogenetischen

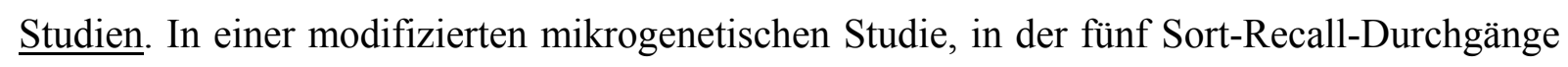
in einer experimentellen Sitzung bearbeitet werden mussten, erwiesen sich alle untersuchten Kinder (Zweit-, Dritt- und Viertklässler) als nutzungsdefizitär. Während ihre Clusterleistung vom ersten zum zweiten Durchgang anstieg, blieb die Wiedergabeleistung über die Durchgänge relativ konstant (Coyle \& Bjorklund, 1996).

Ein sehr differenziertes Bild über die Entwicklung eines effektiven Umgangs mit Strategien lieferten längsschnittliche Analysen. Wie bereits erwähnt war im interindividuellen Vergleich eine große Variabilität zu erkennen, die nicht nur den Entwicklungsverlauf, sondern auch den Zeitpunkt der erstmaligen Anwendung einer Strategie betraf (Schneider \& Sodian, 1997; Sodian \& Schneider, 1999). Hinsichtlich der Effektivität des Strategieeinsatzes 
ließ sich die Entwicklung seit dem Vorschulalter bei $2 / 3$ der Kinder durch eine kontinuierliche Verbesserung der Abrufleistung, bei 1/6 mit einem U-förmigen Verlauf und bei jeweils 1/12 der Kinder durch einen umgekehrt U-förmigen Verlauf oder Leistungsabfall charakterisieren (Sodian \& Schneider, 1999). Unter den Kindern, die ihre Leistung kontinuierlich steigerten, zeigten $40 \%$ der Kinder während dieser Zeit Leistungssprünge, wovon die eine Hälfte diesen Sprung zwischen dem 8. und 10. Lebensjahr, die andere Hälfte zwischen dem 10. und 12. Lebensjahr zeigte. Hinweise auf das Vorliegen eines Nutzungsdefizits gab es demnach nicht generell, sondern nur bei einzelnen Gruppen von Kindern.

Der Strategieerwerb im Vorschul- und frühen Grundschulalter stand scheinbar in keiner Beziehung zu der Abrufleistung, hing aber stark mit der Leistung der älteren Kinder zusammen. Im Vorschulalter verbesserten konsistent nicht-strategische Kinder ihre Behaltensleistung im gleichen Umfang wie die konsistenten Strategen und Strategieentdecker. Die fehlende Strategieeffektivität wurde dabei mit dem fehlenden metakognitiven Verständnis der meisten Kinder in Zusammenhang gebracht.

Neben solchen Befunden lieferten andere Studien aber auch Belege für einen effektiven Strategieeinsatz. Sodian und Schneider (1999) konnten bei Kindern ab dem 8. Lebensjahr einen leistungsdienlichen Strategiegebrauch beobachten. Aus dem bei der Aufgabenbearbeitung gezeigten Organisationsgrad ließ sich zu jedem Messzeitpunkt die Wiedergabeleistung der Kinder vorhersagen, aus der Strategiezunahme wiederum die Reproduktionssteigerung. Im Altersbereich zwischen 8 und 12 waren die individuellen Unterschiede bei der Strategieeffektivität ziemlich stabil.

Der Altersbereich von 6 bis 8 scheint eine Übergangsperiode in Bezug auf den Erwerb und effektiven Gebrauch der Organisationsstrategie darzustellen. Im Alter von 6 Jahren war das Organisationsverhalten kein guter Prädiktor für die Wiedergabeleistung im Alter von 8 Jahren. Die Veränderungen der Anforderungen führen bei den meisten Kinder zu einem Abfall der Wiedergabeleistung zwischen dem 6. und 8. Lebensjahr, unabhängig von der mit 6 Jahren gezeigten Sortierleistung. Im Alter von 8 Jahren gibt es einen Zusammenhang zwischen dem Organisationsverhalten und der Wiedergabeleistung. Das Organisationsverhalten scheint demnach erst um das 8. Lebensjahr herum effektiv zu werden.

Schlagmüller und Schneider (2002) konnten in ihrer mikrogenetischen Studie zeigen, dass auch der erste Strategieeinsatz schon zu einer Steigerung der Reproduktionsleistung führen kann. Die meisten der von ihnen untersuchten Neun- bis Zehnjährigen profitierten 
bereits von der ersten Anwendung der Organisationsstrategien, d.h. verbesserten ihre Erinnerungsleistung. Schlagmüller und Schneider (2002) führten dieses darauf zuück, dass für diese Altersgruppe scheinbar günstige Bedingungen zur Bearbeitung der Anforderungen vorlagen. Ein Nutzungsdefizit wäre demnach entweder bei jüngeren Kindern oder unter ungünstigen Rahmenbedingungen (z.B. Zeitdruck) zu erwarten gewesen.

Neben diesen verschiedenen Studiendesigns sind im Hinblick auf die Erfassung der Effektivität von Strategien verschiedene Phasen des Strategiegebrauchs zu unterscheiden. So kann die Effektivität beim Abruf, bei der Einspeicherung oder im Zusammenwirken beider Stadien erfasst werden, wie Abschnitt 2.2.4 zusammenfasst.

\subsubsection{Anzeichen des Strategiegebrauchs}

Bei der Auswahl von Indizes für das Ausmaß an kategorialem Organisieren lässt sich in Abhängigkeit von der Phase der Aufgabenbearbeitung zwischen Indizes bei der Informationseinspeicherung (in der Lernphase) und Indizes beim Informationsabruf (in der Reproduktionsphase) differenzieren. Dabei ist zu berücksichtigen, dass das Verhalten in der Lernphase nicht unabhängig vom Verhalten in der Abrufphase betrachtet werden kann, da Informationsenkodierung und -abruf in wechselseitigem Zusammenhang stehen.

\section{Anzeichen für kategoriales Organisieren bei der Informationseinspeicherung}

Die Effektivität des kategorialen Organisierens bei der Informationseinspeicherung lässt sich mit Hilfe von „Sort-Recall“-Aufgaben analysieren. Dort zeigte sich, dass Unterschiede in der Gedächtnisleistung vor allem durch Veränderungen des Organisationsverhaltens während der Lernphase bewirkt wurden und weniger durch Prozesse während der Abrufphase. Die Schätzung der Effekte der Lern- und Abruforganisation auf die Gedächtnisleistung über multiple Regressionsanalysen untermauert diese Annahme (Black \& Rollins, 1982; Knopf et al., 1988; Schneider, 1985b, 1986; Schneider et al., 1986).

\section{Anzeichen für kategoriales Organisieren beim Informationsabruf}

Die Effektivität des kategorialen Organisierens beim Informationsabruf wurde häufig mit freien Reproduktionsaufgaben (Lernen und freies Reproduzieren von Wortlisten) überprüft. Dabei wurde geschaut, ob bei der Reproduktion von gelerntem Material eine Tendenz zum Organisieren besteht und ob die Reproduktionsleistung mit dem Organisieren 
zunimmt. Die Ergebnisse bestätigten diese Hypothesen sowohl für das Organisieren mit hineinkonstruierten Ordnungsmöglichkeiten (Jenkins, Mink \& Russell, 1958) als auch für das „subjektive Organisieren“ beim Bearbeiten von Listen mit unzusammenhängenden Wörtern (Tulving, 1962). Die Effektivität der eingesetzten Strategie wurde somit aus dem Zusammenhang zwischen dem Ausmaß des Organisationsverhaltens und der damit einhergehenden Gedächtnisleistung erschlossen.

Darüber hinaus waren systematische Altersdifferenzen beim kategorialen Organisieren beobachtbar (Bousfield, Esterson \& Whitmarsh, 1958). Unter geeigneten Bedingungen waren bereits Zweijährige beim freien Reproduzieren zu kategorialem Organisieren in der Lage (Rossi \& Rossi, 1965). Kinder dieser Altersstufe profitierten in besonderem Maß von Instruktionen, bei denen sie zur Anwendung von Strategien beim Lernen aufgefordert wurden und verbesserten durch die Strategieanwendung ihre Reproduktionsleistung (Kee \& Bell, 1981). Unter geeigneten Rahmenbedingungen scheint der forcierte Einsatz der kategorialen Organisationsstrategie somit auch schon bei sehr jungen Kindern effektiv zu sein.

\section{Zusammenwirken von Einspeicherungs- und Abrufprozessen}

Zur Untersuchung der Wechselwirkung zwischen strategischen Enkodierungs- und Abrufprozessen und ihren Auswirkungen auf die Reproduktionsleistung entwickelten Emmerich und Ackerman (1978) ein experimentelles Design, bei dem Probanden aus unterschiedlichen Altersgruppen (Erstklässler, Fünftklässler, Studenten) auf Bedingungen verteilt wurden, die sich systematisch hinsichtlich der untersuchten Lern- und Abrufstrategien unterschieden. Bei den Lernbedingungen wurde zwischen a) der zufälligen Darbietung des Lernmaterials, b) der Darbietung in kategorialen Blöcken und c) der zufälligen Darbietung des Lernmaterials mit Instruierung zum Sortieren durch die Probanden unterschieden. Die Abrufbedingungen bestanden aus a) dem freien Abruf (Free Recall), b) dem Abruf mit kategorialen Hinweisen und c) dem eingeschränkten Abruf mit kategorialen Hinweisen (d.h. alle Items einer Kategorie mussten wiedergegeben werden, bevor zu einer anderen Kategorie gewechselt werden durfte).

Die Ergebnisse zeigten, dass bei Erst- und Fünftklässlern die Wiedergabe nur durch die Abrufbedingung, aber nicht durch die Lernbedingung beeinflusst wurde. Der maximale Reproduktionswert der Erwachsenen erwies sich hingegen als Funktion der Interaktion der Lern- und Abrufbedingung. Im Gegensatz zu der zufälligen Präsentation der Lernitems konnte in allen Altersgruppen vermehrtes Clustern beobachtet werden, wenn das Lernmaterial nach Kategorien geordnet in Reihen gelegt wurde oder das Lernmaterial zwar randomisiert 
dargeboten wurde, die Kinder aber nach Benennung der Kategorien aufgefordert wurden, die zusammengehörigen Karten zusammenzulegen. Die Lernbedingungen scheinen sich folglich - zumindest bei Kindern - nur auf die Reproduktionsleistung auszuwirken, wenn sie den Gebrauch von Abrufstrategien beeinflussen. Der Einsatz einer Strategie, die in der Lernphase den Nutzen von kategorialen Strukturen garantiert ist im Vergleich zu einer Strategie, die dasselbe beim Abruf tut, relativ uneffektiv. Lernstrategien bekommen mit zunehmendem Alter einen stärkeren Einfluss auf das Erinnerungsvermögen, wenn die Kinder in der Lage sind, die empfohlenen Strategien anzuwenden oder wenn sie selbständig ihre eigenen Abrufstrategien generieren können. An diesem Punkt sorgten das Zusammenwirken von Lern- und Abrufverhalten für eine maximale Wiedergabeleistung.

Weitere Untersuchungen der Enkodierungs-Abruf-Wechselwirkungen zur Schaffung eines Datensatzes für ein Beschreibungs-Modell kamen von Ackerman (1987) und Norman und Bobrow (1979). Es wurde angenommen, dass die Enkodierung von Reiz-Informationen von dem Kontext abhängt, in dem die Kinder mit der Information in Berührung kommen. Die Repräsentation eines Ereignisses (Beschreibung), die entworfen wird, spiegelt nur einen Teil der Information aus dem Orginalreiz wieder, wobei diese Teilinformation durch die beeinflussenden Hinweise bestimmt werden, die während der Enkodierung verfügbar sind.

Die Angemessenheit einer repräsentationalen Beschreibung ist eine Funktion davon, ob sie funktionell bedeutsame Eigenschaften des Reizes enthält, der einen angemessenen Abruf erlaubt. Je nachdem auf welche Attribute mit einer Frage fokussiert wird, kann es eine Erleichterung oder Erschwernis für den Abruf sein.

Ackerman untersuchte mit Kollegen in einer Vielzahl von Studien, ob und in welchem Ausmaß die Leistung von Kindern und Erwachsenen von identischen Lern- und AbrufUmgebungen abhängt (Ackerman, 1982, 1983, 1984, 1985a, 1985b, 1985c, 1985d, 1985e; Ackerman \& Hess, 1982; Ackerman \& Rathburn, 1984; Ackerman \& Rust-Kahl, 1982). Um der Frage nach Entwicklungsunterschieden in der Notwendigkeit vergleichbarer Lern- und Abrufumgebungen nachzugehen, wurde die Art der Fragen, die in der Lern- und in der Abrufphase gestellt wurden, systematisch variiert. Insgesamt war die Stimulus-Wiedergabe bei abweichenden Fragestellungen geringer als bei vergleichbaren Bedinungen. Mit zunehmendem Alter verringerte sich die kontextbedingte Beeinträchtigung, d.h. die Erinnerungsfähigkeit war bei Erwachsenen unter allen Bedingungen vergleichbar gut. Die Qualität der Wiedergabeleistung von jüngeren Kindern hing hingegen noch stark von vergleichbaren Lern- und Abrufhinweisen ab. 
Das Zusammenwirken von Enkodier- und Abrufprozessen wurde auch mit Hilfe von mathematischen Modellen untersucht. Lern- und Behaltensleistungen wurden dabei über die Analyse von Wahrscheinlichkeitsparametern beschrieben, mit Fokussierung auf spezielle Aspekte der Gedächtnisleistung. Die Modelle unterstützten die bisherigen Annahmen bezüglich entwicklungsbedingter Unterschiede in den Lernleistungen weitgehend. Hinweise auf Entwicklungstrends bei den Enkodier- und besonders bei den Abrufparametern fanden sowohl Chechile und Kollegen (Chechile \& Meyer, 1976; Chechile, Richman, Topinka \& Ehrensbeck, 1981; Chechile \& Richman, 1982) als auch Brainerd und Kollegen (Brainerd, 1982; Brainerd \& Howe, 1982; Brainerd, Howe \& Kingma, 1982; Brainerd, Howe, Kingma \& Brainerd, 1984). Unterschiedliche Ansichten gab es dahingehend, ob die Einspeicherung sich schneller entwickelt als der Abruf. Chechile, Richman, Topinka und Ehrensbeck (1981) vertraten diese Auffassung, da in ihren Daten die Einspeicherungsprozesse schon früh auf einem relativ hohes Niveau lagen, während das Abrufniveau bei jüngeren Kindern noch relativ niedrig war und sich bis ins Erwachsenenalter verbesserte. Howe, Brainerd und Kingma (1985a, 1985b) fanden hingegen größere Entwicklungsunterschiede in den AbrufParametern als in den Enkodierungsparametern.

Eine klare Antwort auf die Frage, ob Enkodierungs- oder Wiedergabeprozesse die Gedächtnisentwicklung vorantreiben, lässt sich allerdings nicht treffen, da die Einspeicherungs- und Abrufkomponenten in den verschiedenen Modellen unterschiedliche Beiträge zur Erklärung der Gedächtnisentwicklung liefern. Brainerd (1985) zog aus der vorliegenden Datenlage die Schlussfolgerung, dass der relative Beitrag von Enkodierung und Wiedergabe von der jeweiligen Aufgabe abhängt. Unter „Repeated Recall“ -Anforderungen, bei denen nach einem einzelnen kurzen Lerndurchgang weitere Testdurchgänge durchgeführt wurden, ohne dass die Möglichkeit zum Lernen der vorgegebenen Liste bestand, wurden kaum Entwicklungsveränderungen beim Abruf gefunden (Wilkinson, De Marinis \& Riley, 1983; Wilkinson \& Koestler, 1983, 1984). Bei „Free-Recall“- bzw. „Cued-Recall“-Aufgaben fanden Howe, Brainerd und Kingma (1985a, 1985b), dass der Hinweisreiz bei den jüngeren Kindern die Enkodier-Parameter am stärksten beeinflusste, bei den älteren Kindern hingegen die Abruf-Parameter. Die Erhöhung der Anzahl der taxonomischen Kategorien hatte bei den Zweitklässlern eine positive Auswirkung auf die Abrufleistung in der „Cued-Recall“Bedingung, bei den Viertklässlern auf die Enkodierungs- und Abrufleistung beim „Free Recall“". 


\subsection{Erklärungsannahmen zu Ursachen effektiver Strategieentwicklung}

Wie bereits in Kapitel 2.1 beschrieben, gibt es für die Strategienentwicklung verschiedene Modellvorstellungen, die sowohl Aussagen über ihre Produktion als auch über ihre Effektivität treffen. Hinter diesen Annahmen stehen spezifische Vorstellungen hinsichtlich der zugrunde liegenden Ursachen. Diese beziehen sich auf spezielle Kompetenzen, über die eine Person in einem bestimmten Entwicklungsstadium verfügt, d.h. auf interne Faktoren. Im Folgenden werden die Annahmen der einzelnen Theorien noch näher ausgeführt.

\subsubsection{Annahmen der Mediationstheorie}

In der Mediationstheorie (vgl. Kendler, 1972; Kendler \& Kendler, 1962) wird das klassische Reiz-Reaktions-Schema um einen hypothetischen, sich innerhalb einer Person abspielenden Mediationsmechanismus ergänzt und davon ausgegangen, dass die Konstruktion verbaler intervenierender Mediatoren von Wahrnehmungserfahrungen für die erfolgreiche Bearbeitung von Lern- und Behaltensanforderungen entscheidend ist (vgl. Howe \& Sullivan, 1990). Daraus wurde die Schlussfolgerung abgeleitet, dass erst die kognitiven Voraussetzungen vorhanden sein müssen, bis eine Strategie zum Einsatz kommen kann. Das Ausbleiben bestimmter Verhaltensweisen wurde dabei mit dem Vorliegen konkreter Defizite bei einer Person in Zusammenhang gebracht. Die ausbleibende Strategieproduktion in der Phase des Mediationsdefizits wird darauf zurückgeführt, dass zwischen den Anforderungen (Lernmaterial) und der Gedächtnisleistung (Wiedergabe) keine Beziehung hergestellt wird. Ursache dafür könnte ein wenig ausgereiftes Informationsverarbeitungssystem sein. Die für eine Strategieproduktion notwendigen basalen kognitiven Fähigkeiten und Fertigkeiten scheinen in diesem Entwicklungsstadium noch nicht vorhanden zu sein (Reese, 1962). Wenn die erforderlichen Voraussetzungen vorhanden sind, führt dieses dennoch nicht zum sofortigen Einsatz von Strategien, sondern erfordert in der Phase des Produktionsdefizits konkrete Hilfestellungen (Flavell, 1970).

Eine mögliche Erklärung für das Auftreten eines Produktionsdefizits ist ein Fehler in der Einspeicherung oder ein Unvermögen, das Lernmaterial in der Lernphase eindeutig und dauerhaft zu gruppieren. Lange und Griffith (1977) favorisieren diese Erklärung aufgrund des starken Zusammenhangs zwischen Input-Organisation und Wiedergabe. Alternativ dazu oder 
ergänzend könnte die Ursache allerdings auch in der Abrufphase zu finden sein, in der Gruppierungen, die in der Lernphase entstanden sind, nicht genutzt werden können.

Die Grundlage eines Produktionsdefizit kann eine unzureichende mentale Kapazität (Schneider 2000) und fehlendes bereichsspezifisches Wissen (Schneider, 2000; Waters, 2000) sein.

Unter Rückgriff auf die Erklärungsansätze der Mediationstheorie lässt sich die Entwicklung des effektiven Strategiegebrauchs allerdings nicht abschließend beantworten. Daher ist es notwendig, sich mit neueren Erklärungsansätzen zu beschäftigen, in denen explizit auf die Bedeutung einzelner Determinanten für die Entwicklung effektiver Strategien eingegangen wird.

\subsubsection{Automatische Wissensaktivierung (Bjorklund)}

Für Bjorklund $(1985,1987)$ bestehen die entscheidenden Veränderungen während der Entwicklung des kategorialen Wissens in der Zunahme der Anzahl von Items und der Anzahl und Stärke der Interitem-Relationen, wodurch der Zugriff auf die vorhandenen Informationen leichter wird und Merkmale enkodiert werden können (vgl. Ackerman, 1984; Ceci, 1980; Ceci \& Howe, 1978b). Danach können bei einer reichhaltigen Wissensbasis auch automatische Prozesse für das kategoriale Organisieren von Vor- und Grundschulkindern verantwortlich sein. Eine Steigerung der Gedächtnisleistung wäre damit nicht das Verdienst des Kindes, das ein bestimmtes Verhalten gezielt eingesetzt hat, um dadurch seine Erinnerungsfähigkeit zu erhöhen, sondern ein Nebenprodukt des normalen Informationsverarbeitungsprozesses. Da es sich also definitionsgemäß nicht um eine Strategie handelt (vgl. Kap. 2.1.1), kann bei einer Leistungssteigerung auch nicht von effektiven Verhaltensweisen, sondern höchstens von effektiven Verarbeitungsprozessen gesprochen werden. Während einige Forscher die bestehenden assoziativen Relationen der Wissensbasis etwas allgemeiner als Basis für die anschließenden Prozesse ansehen (Lange, 1973; Haynes \& Kulhavy, 1976), sehen andere eine direkte Beziehung zwischen dem Ausmaß des kategorialen Organisierens und der Stärke der Interitem-Relationen (Chi, 1978, 1985; Chi \& Koeske, 1983).

Darüber hinaus postuliert Bjorklund qualitative Veränderungen in der Merkmalshierarchie, die die Verarbeitung eines Items determiniert (Schwanenflugel, Guth \& Bjorklund, 1986). Für das kategoriale Reproduzieren bei Reproduktionsanforderungen bedeutet dieses, dass bis zum 12. Lebensjahr kategoriales Organisieren nur aufgrund von 
automatischer Aktivationsausbreitung zustande kommt, wenn die Interitem-Relationen des Lernmaterials stark genug sind. Um das 13. Lebensjahr sind die Kinder in der Lage, über das eigene Verhalten zu reflektieren und gezielt nach kategorialen Relationen zu suchen, was dem Konzept der reflektiven Abstraktion (Piaget, 1971) nahe kommt. Strategisches Organisieren im Sinne einer bewussten und planvollen Nutzung der Strategie ist damit erst nach dem 13. Lebensjahr zu erwarten.

\section{Argumente für die Theorie von Bjorklund}

Die Bedeutung der Wissensbasis für die Entwicklung effektiver Strategien äußert sich darin, dass sich mit zunehmendem Alter die Anzahl von Informationen (Knoten) und die Quantität und Qualität der Beziehungsstrukturen (Verknüpfungen zwischen den in der Wissensbasis repräsentierten Informationen) verbessert. Von dem bereichsspezifischen und strukturellen Wissen sollte nun ein direkter und unbewusster Einfluss auf die Gedächtnisleistung erfolgen.

Dieser Einfluss basiert vor allem auf dem zunehmend leichteren Zugriff auf spezifische Items (Item-spezifische Effekte) in den reichhaltigeren Repräsentationen des semantischen Netzwerkes (Chechile \& Richman, 1982; Ghatala, 1984; Ornstein, Hale \& Morgan, 1977) und der fast automatischen Aktivierung von Verbindungen zwischen den Item-Sets, also nicht-strategischen Organisationsprozessen (Bjorklund \& Bjorklund, 1985; Bjorklund \& Zeman, 1982). Bei für die jeweilige Kategorie typischem Material (Bjorklund, 1988; Rabinowitz, 1984; Rabinowitz \& Kee, 1994) oder stark miteinander assoziierten Items (Bjorklund \& Jacobs, 1985; Frankel \& Rollins, 1985; Schneider, 1986) kann kategoriales Organisieren demnach auch ohne strategisches Bewusstsein erfolgen.

Bei jüngeren Kindern haben Faktoren der Wissensbasis noch einen wesentlich stärkeren Einfluss auf den Einsatz von Gedächtnisstrategien als bei älteren Kindern. Dieses zeigt sich z.B. beim Erwerb von Organisationsstrategien an den Alters- und Typizitätseffekten des verwendeten Materials. Bei Listen mit kategorial typischen Items wenden alle Kinder eher eine Strategie an und übertragen sie auf andere Anforderungen, wobei ältere Kinder den jüngeren grundsätzlich überlegen sind. Die Leistung der jüngeren Kinder ist noch weitgehend abhängig vom Typizitätsniveau des Lernmaterials, d.h. bei untypischen Items fallen ihnen der Einsatz und die Aufrechterhaltung einer Strategie wesentlich schwerer (Bjorklund \& Buchanan, 1989). 
Wie bereits beschrieben kann kategoriales Organisieren bei für eine Kategorie typischem Material (Bjorklund, 1988; Rabinowitz, 1984; Rabinowitz \& Kee, 1994) oder stark miteinander assoziieren Items (Bjorklund \& Jacobs, 1985; Frankel \& Rollins, 1985; Schneider, 1986) auch ohne strategisches Bewusstsein erfolgen. Darüber hinaus erleichtert eine reichhaltige Wissensbasis aber auch die Nutzung von bewussten Strategien (Chi \& Ceci, 1987; Hasselhorn, 1995; Rabinowitz \& Chi, 1987). Mit zunehmender Erfahrung können auch komplexe Strategien automatisiert und auf neue Situationen angewendet werden (Best, 1993; Rabinowitz, Freeman \& Cohen, 1992).

Vertrautheit mit dem Lernmaterial und die dem Kind verfügbaren kategorialen und /oder assoziativen Interitemrelationen spielen eine entscheidende Rolle für die Anwendung einer Strategie, bei der nach Kategorien organisiert wird. Bei Konfrontation mit unvertrautem Material, für das in der Wissensbasis keine „Knoten“ existieren, ist der Verweis auf entsprechende Relationen sinnlos. Andererseits sind höher kontrollierte strategische Organisationsprozesse überflüssig, wenn ein eng umschriebener Ausschnitt der eigenen Wissensbasis sehr strukturiert repräsentiert und dabei hochgradig überlernt ist.

\subsubsection{Strategische Wissensaktivierung (Hasselhorn)}

Der strategische Rückgriff auf das eigene kategoriale Wissen ist für Hasselhorn (1996) auch schon zwischen dem 8. und 10. Lebensjahr möglich. Seine Kritik an dem Erklärungsansatz von Bjorklund richtet sich an die Vernachlässigung verschiedener Einflussfaktoren, wie z.B. dem aufgabenspezifische Metagedächtnis oder dem Aufgabenkontext. Außerdem sieht er die verfügbaren mentalen Ressourcen als Rahmenbedingung aber nicht als direkte Ursache für den Einsatz kategorialer Organisationsstrategien (vgl. Mitchell \& Hunt, 1989). In seinem integrativen Rahmenmodell, der Strategie-Emergenz-Theorie, erklärt er die Informationsaufnahme mit dem Arbeitsgedächtnismodell von Baddeley (1986), das Systemeigenschaften und Verarbeitungsmechanismen postuliert. Die Art der Repräsentation von Wissen im Langzeitgedächtnis und damit verbundene Abrufmechanismen werden mit Hilfe des semantischen Netzwerkes dargestellt und unter Einbeziehung von Tulvings „Synergistic Ecphory“-Modell (Tulving, 1982) noch näher spezifiziert. Den entscheidenden Motor für die Entwicklung effektiver strategischer Gedächtnisprozesse sieht Hasselhorn (1996) in einem gut ausgebildeten Metagedächtnis. Abbildung 2.4 veranschaulicht die in der StrategieTheorie-Emergenz-Theorie postulierten Zusammenhänge. 
Kontext:

Umgebung Lernmaterial Aufgabenstellung Instruktionen Hilfestellung

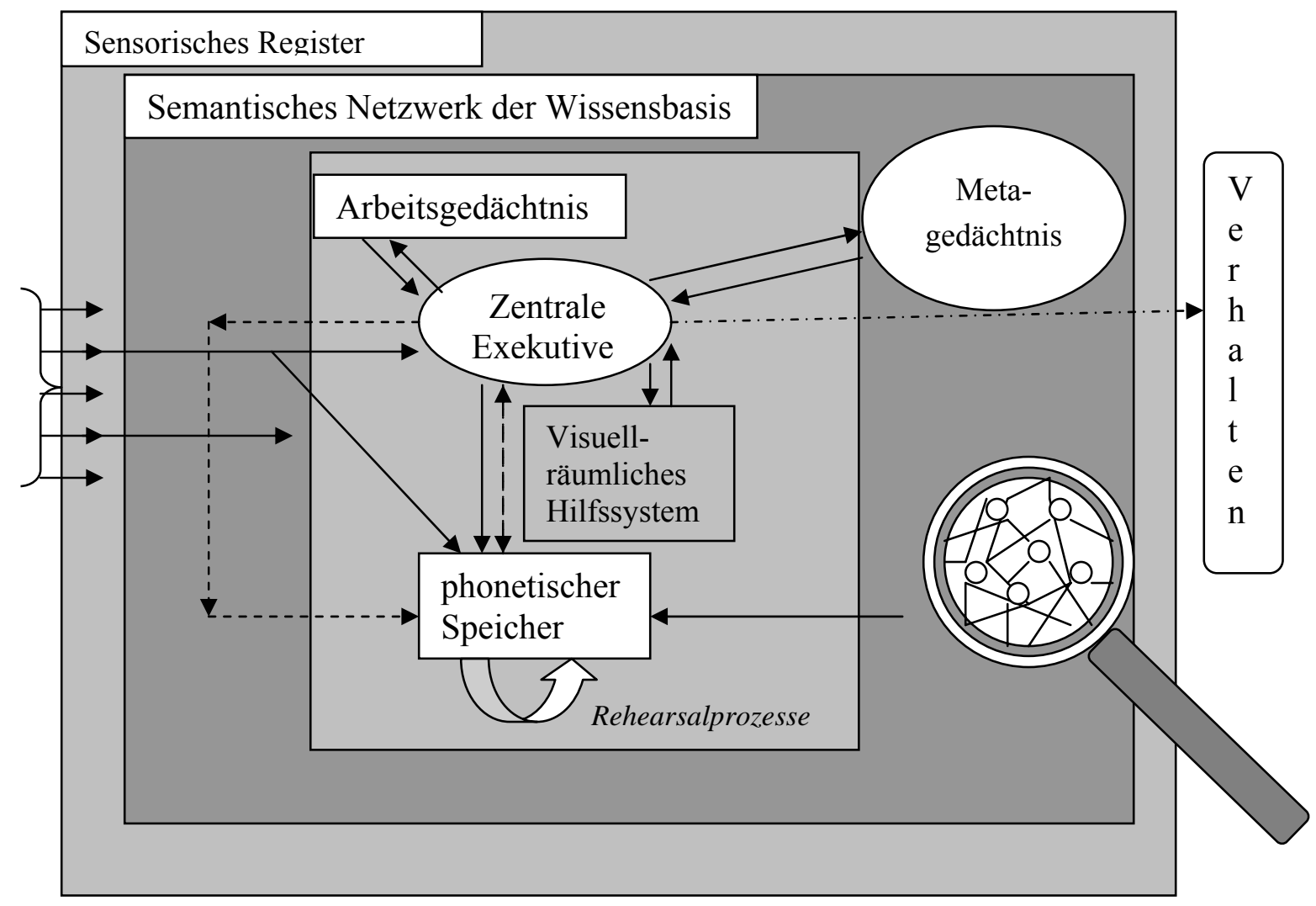

\begin{tabular}{|l|l|}
\hline Ekphorische Prozesse & \\
\hline Konversionsprozesse &
\end{tabular}

Abbildung 2.4: Integratives Rahmenmodell der Informationsverarbeitung (Hasselhorn, 1996, S. 71)

\section{Belege für die Strategie-Emergenz-Theorie}

Für die Annahme, dass das automatische Aktivieren kategorialen Wissens in der zweiten Hälfte der Grundschuljahre von einer strategischen Wissensnutzung abgelöst wird, lieferte Hasselhorn (1990a, 1990b, 1996; Hasselhorn \& Lindner-Müller, 1995) eine Vielzahl von Befunden. Neben steigenden Organisationstendenzen beim Einprägen und Abrufen von Lerninhalten und zunehmenden metakognitiven Kompetenzen zeichnete sich dieser Altersabschnitt durch eine weitaus höhere Stabilität des Verhaltens und Wissens aus.

Auch für die Annahme, dass ein elaboriertes Wissen über Gedächtnisstrategien (Metagedächtnis) die entscheidende Grundlage für kategoriales Organisieren ist, gab es empirische Evidenz. Hasselhorn (1990b; Hasselhorn \& Lindner-Müller, 1995) fand am Ende der Grundschulzeit einen Zusammenhang zwischen kategorialem Organisieren und metamemorialen Kompetenzen. Insgesamt war zwischen der ersten und zweiten Hälfte der 
Grundschulzeit eine Zunahme der Prädiktionskraft von Input-Organisation, OutputOrganisation und (distalem) Metagedächtnis für die Vorhersage der Reproduktionsleistung zu verzeichnen. $\mathrm{Ab}$ der dritten Klassenstufe erwies sich der Einfluss des distalen Metagedächtnis auf die Reproduktionsleistung als bedeutsam. Untermauert wurde dieser Befund durch Pfadanalysen, mit denen bei Viertklässlern die gerichteten Zusammenhänge zwischen Metagedächtnis, Erkennen der kategorialen Listenstruktur, kategorialem Organisieren und der Reproduktionsleistung nachweisbar waren. Die Reproduktionsleistung der Zweitklässler wurde hingegen vor allem durch ihr materialspezifisches Vorwissen bestimmt (Hasselhorn, 1995, 1996).

Auch andere Untersuchungen lieferten Belege für die intentionale Nutzung von kategorialem Wissen am Ende der Grundschulzeit (d.h. mit 10 Jahren). So gelang älteren Grundschülern im Gegensatz zu jüngeren Kindern auch die Gruppierung von niedrigassoziativen Items (Schneider, 1986). Bei der Durchführung von mehreren Lerndurchgängen, gab es in den späteren Durchgängen eine zunehmende Anzahl von Viertklässlern, die Strategien anwendeten (Bjorklund, 1988).

Darüber hinaus kommt dem Metagedächtnis eine entscheidende Rolle zu für das Beibehalten einer Gedächtnisstrategie. Bei deutschen und amerikanischen Kindern konnte dieser Zusammenhang zwischen dem Wissen und dem Verhalten bei der Aufgabenbearbeitung auch noch 6 Monate nach dem Training gezeigt werden (Carr, Kurtz, Schneider, Turner \& Borkowski, 1989). Diese Befunde konnten auch mit anderen methodischen Herangehensweisen (Videopräsentationen), bei denen Unterschiede in verbalen Kompetenzen weniger ins Gewicht fielen, bestätigt werden (Justice, 1985, 1986; Schneider, 1986; Schneider, Körkel \& Vogel, 1987).

Hinsichtlich der Effektivität des Strategieeinsatzes konnte Hasselhorn (1990b) zeigen, dass es am Ende der Grundschulzeit bei Sort-Recall-Aufgaben bedeutsame Übereinstimmungen zwischen dem Organisationsverhalten in der Lern- und Abrufphase, sowie dem strategischen Verhalten und der Erinnerungsleistung gab. Die Prädiktorfunktion von Organisationsprozessen für die Gedächtnisleistung fanden auch Black und Rollins (1982) und Schneider (1986) in ihren Regressionsanalysen. Während bei den jüngeren Kindern das Verhalten in der Abrufphase (Clusterverhalten) allerdings den größeren Erklärungswert aufwies, war es bei älteren Kindern das Verhalten in der Lernphase (Sortierverhalten).

Weitere Hinweise dafür, dass am Ende der Grundschulzeit gezielt Kategorisierungsstrategien eingesetzt werden, da den Kindern die Bedeutung der Organisationsstrategien für eine erfolgreiche Wiedergabe bewusst ist, liefert die 
Untersuchung von Schneider (1986). Aus dem Sortierverhalten in der Lernphase und dem Vorhandensein eines aufgabenbezogenen Metagedächtnisses ließen sich bei den Viertklässlern die besten Vorhersagen für die Wiedergabeleistung treffen (Schneider, 1986).

Ein effektiver Strategiegebrauch scheint demnach $\mathrm{zu}$ jeder Zeit in engem Zusammenhang mit Wissensaspekten zu sehen. Während diese aber zu Beginn der Grundschulzeit eher auf basaler Ebene anzusiedeln sind, müssen am Ende der Grundschulzeit höhere kognitive Wissensinhalte (= metakognitives Wissen) betrachtet werden.

\subsubsection{Kritische Reflektion der Erklärungsmodelle}

Die unterschiedlichen Erklärungsmodelle beleuchten leider nur einzelne Aspekte des strategischen Verhaltens und vernachlässigen dabei das komplexe Wirkungsgeflecht interner und externer Faktoren. Bevor diese Modelle für die Erklärung der Strategieeffektivität herangezogen werden, muss daher noch einmal auf ein paar wesentliche Aspekte eingegangen werden.

\section{Automatische Wissensaktivierung (Bjorklund)}

Bjorklund $(1985,1987)$ führte kategoriales Organisieren bis zum 12. Lebensjahr in erster Linie auf automatische Aktivationsausbreitung zurück, die mit den Interitem-Relationen des Lernmaterials zusammenhängen. Strategisches Organisieren im Sinne einer bewussten und planvollen Nutzung der Strategie erwartet er erst nach dem 13. Lebensjahr, da seiner Meinung nach erst dann Kinder ihr Verhalten reflektieren und gezielt nach kategorialen Relationen suchen.

Hier ist jedoch kritisch anzumerken, dass die Art der Beziehung zwischen Wissensbasis und strategischem Verhalten schwer $\mathrm{zu}$ spezifizieren ist. Während einige Forscher die bestehenden assoziativen Relationen der Wissensbasis etwas allgemeiner als Basis für die anschließenden Prozesse ansehen (Lange, 1973; Haynes \& Kulhavy, 1976), sehen andere eine direkte Beziehung zwischen dem Ausmaß des kategorialen Organisierens und der Stärke der Interitem-Relationen (Chi, 1978, 1985; Chi \& Koeske, 1983). Nach Einschätzung von Hasselhorn (1996) kommt der Wissensbasis unter den Voraussetzungen, dass weder unvertrautes noch hoch überlerntes Lernmaterial präsentiert wird und dass die Gedächtnisanforderungen unter hinreichend unterstützenden Aufgabenbedingungen gestellt werden, ab dem 10. Lebensjahr eine eher unterstützende Funktion zu. Der prinzipiell strategisch ausgerichtete Suchprozess in der eigenen Wissensbasis kann umso effizienter 
ablaufen, je stärker die kategorialen Relationen eines Items bzw. die intrakategorialen Interitem-Assoziationen ausgeprägt sind. Bei einer größeren Effizienz der Abrufprozesse stehen wiederum mehr Ressourcen für andere Informationsverarbeitungsschritte zur Verfügung, was sich positiv im Sinne einer Leistungssteigerung auswirken kann, also die Entwicklung effektiver strategischer Aktivitäten begünstigt. Mit Hilfe einer ausgereiften Wissensbasis lassen sich mangelnde Fähigkeiten zwar zum Teil ausgleichen, aber nicht vollständig als Störfaktor im Hinblick auf die effektive Strategienutzung eliminieren. Beim Vergleich von Fußball-,Experten“ und Laien in der zweiten und vierten Klasse zeigte sich, dass die Experten mit niedrigem IQ in einer Sort-Recall-Aufgabe ähnliche Leistungen zeigten wie Laien mit hohem IQ. Unter den Fußball-,Experten“ gab es allerdings in Abhängigkeit vom Intelligenzniveau deutliche Unterschiede (Schneider \& Bjorklund, 1992).

\section{Strategische Wissensaktivierung (Hasselhorn)}

In dem Modell beeinflussen externe Faktoren (Umgebung, Lernmaterial, Aufgabenstellung, Instruktionen, Hilfestellungen) als „Kontext“ die internen Prozesse während der Informationsaneignung und während des Abrufs. Die dargebotenen Informationen gelangen in das sensorische Register (Peripherie des internen Gedächtnissystems). Dabei geht ein Großteil verloren (,Spurenzerfall“), ein anderer Teil gelangt ohne bewusste Kontrolle ins semantische Netzwerk der Wissensbasis, wo entsprechende Repräsentationsknoten aktiviert werden (=automatische Enkodierprozesse). Der dritte Teil der aufgenommenen Informationen wird bewusst verarbeitet, d.h. er gelangt in den von der zentralen Exekutive kontrollierten Bereich des Arbeitsgedächtnisses, zieht Aufmerksamkeit auf sich und wird in den phonetischen Speicher des Arbeitsgedächtnisses eingelesen. Mit Hilfe von Rehearsalprozessen kann dort die Information aktiv (=zugänglich) gehalten werden. Je länger diese Information auf der phonetischen Schleife memoriert wird, desto stärker wird der entsprechende Repräsentationsknoten im semantischen Netzwerk aktiviert. Darüber hinaus findet auch eine qualitative Verbesserung statt. Bei hinreichend entwickeltem Metagedächtnis greifen auch elaboriertere Kontrollmechanismen (z.B. kumulatives Rehearsal) in die Verarbeitungsprozesse ein. Wird in der Verarbeitungsphase beim Lernmaterial eine kategoriale Struktur erkannt, so können weitere zentral-exekutive Kontrollaktivitäten initiiert werden (z.B. verstärkte Mitaktivierung von mit dem Repräsentationsknoten eines Items verbundenen kategorialen Relationen). Die Qualität der Kontroll- und Steuerfunktionen (der zentralen Exekutive innerhalb des Arbeitsgedächtnisses) hängt demnach wesentlich vom Entwicklungsstand des deklarativen Metagedächtnisses ab. 
Umgekehrt können Kontrollaktivitäten der zentralen Exekutive aber auch zur Weiterentwicklung des deklarativen Metagedächtnisses führen. In der Abrufphase aktivieren ekphorische Prozesse, die von der zentralen Exekutive des Arbeitsgedächtnisses überwacht bzw. kontrolliert werden, potentiell relevante Ausschnitte des semantischen Netzwerkes und geben sie als ekphorische Information zur weiteren Verarbeitung in das Hilfssystem der phonetischen Schleife. Konversionsprozesse prüfen daraufhin, welche Informationen relevant sind (d.h. zur vorher dargebotenen Lernliste gehören und noch nicht reproduziert wurden). Die als relevant eingestuften Informationen werden schließlich für den Output freigegeben und können in offenes Antwortverhalten umgesetzt werden.

Für Hasselhorn (1996) ist die entwicklungsbedingte Zunahme der Gedächtnisleistung in erster Linie auf die verbesserten strategischen Kompetenzen zurückzuführen. Eine angemessene Strategieanwendung wird dabei durch ein gut ausgebildetes Metagedächtnis möglich. Im weiteren Entwicklungsprozess tragen das metakognitive Wissen, der Einsatz von Strategien und die daraus resultierende Leistungssteigerung wechselseitig zur Verbesserung der Effektivität des Strategiegebrauchs bei.

Diese in dem Erklärungsmodell postulierten Zusammenhänge fielen allerdings nicht auf ungeteilte Zustimmung, sondern wurden teilweise kritisch diskutiert. Ein Kritikpunkt richtete sich beispielsweise gegen die Bedeutung, die strategischem Verhalten als Entwicklungsdeterminante für die Gedächtnisleistung zugesprochen wurde. Die kognitiven Leistungsverbesserungen von der frühen Kindheit bis zur Adoleszenz werden durch diverse entwicklungspsychologische Studien belegt. Der steile Anstieg zwischen dem 6. und 11. Lebensjahr bei Reproduktionsleistungen unterschiedlicher Art ließ sich allerdings nur schwer alleine mit der zunehmenden Verarbeitungsgeschwindigkeit erklären (Bryan \& Luszcz, 1996), so dass nach weiteren Einflussfaktoren gesucht wurde. Neben strategischen Einflüssen wird allerdings auch eine Vielzahl anderer Faktoren als Determinanten der Gedächtnisentwicklung diskutiert (vgl. Reyna \& Brainerd, 1989). Dempster (1981, 1985) führt die alterskorrelierte Zunahme der Gedächtnisspanne in erster Linie auf eine kürzere Itemidentifikationsgeschwindigkeit zurück. Bjorklund $(1985,1987)$ betont, wie bereits erwähnt, besonders die Bedeutung der Wissensbasis, die bei der Verarbeitung von semantischem Material zumindest bis zum Jugendalter auch durch automatische Aktivierungsprozesse zu Leistungsverbesserungen führen kam.

Ein weiteres Argument gegen die Notwendigkeit strategischen Verhaltens für die Güte von Gedächtnisleistungen ist die z.T. ausbleibende Leistungsverbesserung nach dem erstmaligen Strategieeinsatz (Miller \& Seier, 1994) oder die mangelnde Stabilität nach 
gezieltem Strategietraining (Bjorklund et al., 1997). Das Konzept des Nutzungsdefizits wird in diesem Zusammenhang von Reyna (1996) als Versuch gewertet, eine fehlerhafte Überzeugung zu rechtfertigen: ,The construct of utilization deficiency serves a role similar to that of horizontal decalage in Piagetian theory. It acknowledges a disconfirmation of the main theoretical claim, namely that strategies cause memory performance to improve. Rather than labelling failures to obtain effects of strategies as utilization deficiencies, it may be more straightforward to consider them to be null effects“ (Reyna, 1996; S. 92).

Als wesentlich bedeutsamer wird stattdessen von einigen Forschern der Einfluss basaler kognitiver Prozesse eingeschätzt, was in Aussagen wie „...the development of recall is controlled by basic rather than strategic processes“ (Brainerd, 1995; S. 136) oder „...the development of recall is governed chiefly by age changes in basic processes, especially age changes in production of and sensitivity to different forms of interference“ (Brainerd, 1995; S. 137) deutlich wird. Aber auch motivationalen Faktoren wird ein entscheinender Stellenwert eingeräumt (Naus, Ornstein \& Aivano, 1977; Kunzinger \& Witryol, 1984).

\subsection{Determinanten für die Produktion und Effektivität von Strategien}

Wenn hinsichtlich der Entwicklung des Strategiegebrauchs und seiner Effektivität unterschiedliche Befunde berichtet werden, so ist die Ursache dafür nicht ausschließlich in der Wahl unterschiedlicher Klassifikationskriterien für strategisches Verhalten zu suchen.

Bei der Auseinandersetzung mit dem Phänomen des Nutzungsdefizits war bereits darauf hingewiesen worden, dass Altersunterschiede und die Erfahrung im Umgang mit Strategien von großer Bedeutung für die Qualität des Strategiegebrauchs sind. Darüber hinaus sind eine Reihe weiterer Faktoren denkbar, die den Einsatz und den Nutzen von Strategien beeinflussen. Diese können sich wiederum wechselseitig fördern oder hemmen und bei unterschiedlichen Individuen und Rahmenbedingungen einen anderen Stellenwert für die Ausbildung effektiver Strategien haben. In diesem Zusammenhang wurde neben der

Bedeutung von kulturellen Aspekten, wie elterlichen Erziehungspraktiken und dem Bildungssystem (Carr, Kurz, Schneider, Turner \& Borkowski, 1989; de la Mata \& Sanchez, 1991) auch die Bedeutung von Persönlichkeitsfaktoren diskutiert (Bjorklund, Miller, Coyle \& Slawinski, 1997), auf die an dieser Stelle aber nicht weiter eingegangen werden soll.

Darüber hinaus wurde die Abhängigkeit vom Geschlecht (z.B. Cox \& Waters, 1986; Waters \& Schreiber, 1991) und von Intelligenzunterschieden thematisiert (z.B. Gaultney, Bjorklund \& Goldstein, 1996, Schneider, Bullock \& Sodian, 1998). Bjorklund et al. (1994) 
fanden bei trainierten Kindern unabhängig von der Intelligenz ähnliche Ergebnismuster für Wiedergabe, Sortierleistung und Clustern. Besonders hohe Wiedergabeleistungen wurden in der Gruppe der perfekten Sortierer von den Kindern mit hohem Intelligenzquotient (IQ) erzielt. Insgesamt schienen nur Kinder mit einem hohen IQ ein Nutzungsdefizit aufzuweisen, und dieses bezog sich meistens auch nur auf die Cluster-Messung. Kinder mit hohem IQ profitieren mehr vom Gebrauch einer Strategie als weniger intelligente Kinder. Bei bestimmten Teilstrategien weisen sehr intelligente Kinder vermutlich schon früher ein Nutzungsdefizit auf als weniger intelligente Kinder. Das Hauptinteresse lag allerdings meistens auf der Rolle spezifischer interner und externer Faktoren und ihrem Zusammenspiel. Bei den internen Faktoren lassen sich kognitive (Wissensbasis, Arbeitsgedächtniskapazität, Metagedächtnis) von nicht-kognitiven Aspekten (z.B. Motivation, Selbst-Attribution, erwartete Selbstwirksamkeit) abgrenzen (Borkowski \& Turner, 1990; Bandura, 1989).

Die Ursachen für das Auftreten eines Nutzungsdefizits können in unterschiedlichen Stadien der Strategieineffizienz (vgl. Miller \& Seier, 1994), bei unterschiedlichen Strategien und in unterschiedlichen Altersstufen sehr verschieden sein. In einer sehr frühen Phase des Nutzungsdefizits stellt sich die Frage, warum Kinder eine Strategie produzieren und beibehalten, wenn sie ihnen nicht hilft. In den späteren Phasen kann man sich fragen, warum die Strategien älteren Kindern mehr nutzen als jüngeren. Die Kombination von mehreren Strategien kann bei einigen Strategien von Bedeutung sein (z.B. bei einer Selektionsstrategie), bei anderen spielt sie eher eine marginale Rolle (z.B. bei einer Rehearsalstrategie). Da in der Regel nur jüngere Kinder Probleme bei der Hemmung schlechter Strategien bzw. Verhaltensweisen und einen unrealistischer Optimismus hinsichtlich der künftigen Leistung zeigen, werden nur sie durch diese Einflussfaktoren beeinträchtigt werden. Die Schwierigkeiten älterer Kinder bei der Bewältigung von strategischen Anforderungen dürften dagegen eher mit einer zu kleinen Wissensbasis und einem unangemessenen Metagedächtnis zusammenhängen.

Darüber hinaus haben die Einflussfaktoren eine spezifische Wirkung auf die unterschiedlichen Entwicklungsstadien bei der Strategieanwendung, also

- die Bewertung der Strategie,

- die Anbahnung der Strategie,

- die Durchführung der Strategie,

- das Beibehalten (oder Verwerfen) einer Strategie und

- die Verarbeitung des zu behaltenden Materials. 
Bei der Strategiebewertung, -einleitung und -durchführung spielen sowohl die Gedächtniskapazität, das Wissen und das Metagedächtnis als auch die Fähigkeit zur Hemmung früherer Behaltensweisen eine Rolle. Die metakognitiven Kompetenzen und die Qualität der Unterdrückung konkurrierender Verhaltensweisen beeinflussen anschließend, ob die Strategie beibehalten oder aufgegeben wird. Für die Verarbeitung der zu behaltenden Items kann neben dem Einfluss der Gedächtniskapazität, des Wissens und des Metagedächtnisses auch die Fähigkeit zur Integration einer Strategie in andere Gedächtnisaktivitäten wichtig sein. Eine Übersicht über die Annahmen zur Phasenspezifität der Bedeutung der verschiedenen Einflussfaktoren bietet Tabelle 2.4.

Tabelle 2.4: Determinanten für effektive Strategieproduktion (in Anlehnung an Miller \& Seier, 1994)

\begin{tabular}{|c|c|c|c|c|c|c|}
\hline \multirow[b]{3}{*}{ Phase } & \multicolumn{6}{|c|}{ Determinanten } \\
\hline & \multirow{2}{*}{$\begin{array}{l}\text { Gedächtnis- } \\
\text { kapazität }\end{array}$} & \multirow[t]{2}{*}{ Wissen } & \multirow{2}{*}{$\begin{array}{l}\text { Meta- } \\
\text { gedächtis }\end{array}$} & \multicolumn{2}{|c|}{ Zentrale Exekutive } & \multirow{2}{*}{\begin{tabular}{|l} 
Moti- \\
vation / \\
Selbst- \\
konzept
\end{tabular}} \\
\hline & & & & $\begin{array}{l}\text { Hemmungs- } \\
\text { kompetenz }\end{array}$ & $\begin{array}{l}\text { Integrations- } \\
\text { fähigkeit }\end{array}$ & \\
\hline $\begin{array}{l}\text { Bewertung der } \\
\text { Strategie }\end{array}$ & $\mathrm{X}$ & $\mathrm{X}$ & $\mathrm{X}$ & $\mathrm{X}$ & & \\
\hline $\begin{array}{l}\text { Entscheidung für } \\
\text { die Strategie }\end{array}$ & & & & & & $X$ \\
\hline $\begin{array}{l}\text { Anbahnung der } \\
\text { Strategie }\end{array}$ & $\mathrm{X}$ & $\mathrm{X}$ & $\mathrm{X}$ & $\mathrm{X}$ & & \\
\hline $\begin{array}{l}\text { Durchführung } \\
\text { der Strategie }\end{array}$ & $\mathrm{X}$ & $\mathrm{X}$ & $\mathrm{X}$ & $\mathrm{X}$ & & \\
\hline \begin{tabular}{|ll} 
Beibehalten & vs. \\
Aufgeben & der \\
Strategie & \\
\end{tabular} & & & $\mathrm{X}$ & $\mathrm{X}$ & & $X$ \\
\hline $\begin{array}{l}\text { Informations- } \\
\text { verarbeitung }\end{array}$ & $\mathrm{X}$ & $\mathrm{X}$ & $\mathrm{X}$ & & $\mathrm{X}$ & \\
\hline
\end{tabular}

Leider existieren bisher keine konsistenten Befunde über die differentielle Wirkung der einzelnen Faktoren in den jeweiligen Phasen. Auch wenn kognitive Faktoren einen erheblichen Stellenwert bei der effektiven Strategieentwicklung einnehmen, können sie nicht 
unabhängig von anderen Einflussfaktoren betrachtet werden. Eine besondere Bedeutung kommt in diesem Zusammenhang motivationalen Faktoren zu (vgl. Borkowski \& Muthukrishna, 1995; Schneider \& Pressley, 1989).

Allerdings lassen sich die beschriebenen Konstrukte nicht klar voneinander trennen. So gibt es viele Überschneidungsbereiche, z.B. zwischen der Wissensbasis und motivationalen Aspekten. So ist es z.B. denkbar, dass bei einer größeren Wissensbasis und einem besseren Metagedächtnis bessere Erfolge beim Erinnern erzielt werden, was rückwirkend eine Steigerung der Motivation zur Folge hat. Zunächst sollen jedoch die kognitiven Faktoren näher beleuchtet werden, zu denen wie bereits erwähnt u.a. die Gedächtniskapazität, das Vorwissen und das metakognitive Wissen zählen.

\subsubsection{Arbeitsgedächtniskapazität}

Die Tatsache, dass unsere kognitiven Leistungen manchmal an ihre Grenzen stoßen, wird von Psychologen meistens auf die Begrenztheit der kognitiven Ressourcen und in dem Zusammenhang vor allem auf die Kapazität des Arbeitsgedächtnisses zurückgeführt. Die Grundlage für diese Erklärung der Effektivität unserer Gedächtnisprozesse bilden die Mehrspeichermodelle, in denen das Gedächtnis in verschiedene Komponenten unterteilt wird. Für jeden dieser Speicher werden charakteristische Invarianten postuliert, die die Qualität der enthaltenen Informationen (Kodierungsart: phonologisch, semantisch), die Verweildauer der aufgenommenen Informationen (Zykluszeit, Verfallszeit) und die Quantität der verbleibenden Informationen (Speicherkapazität) betreffen (vgl. Atkinson und Shiffrin, 1968). Während die Kapazität des sensorischen Registers und des Langzeitspeichers keinen Einschränkungen unterliegt, ist die Kapazität des Kurzzeitspeichers begrenzt (Miller, 1956). Schwierigkeiten bei der Verarbeitung und Abspeicherung von Informationen, die nicht auf die Art der Kodierung oder zeitliche Faktoren zurückgeführt werden können, werden daher auf das Kurzzeitgedächtnis bzw. Arbeitsgedächtnis (Baddeley \& Hitch, 1974) zurückgeführt.

Die Speicherkapazität des Kurzzeitspeichers wurde eine Zeit lang auf „sieben plus/minus zwei“ Informationseinheiten beziffert (Miller, 1956). Zweifel an dieser als fix angesehenen Grenze tauchten auf, als in späteren Untersuchungen auch höhere Behaltensleistungen beobachtet werden konnten. Diese Steigerung der Behaltensleistung wurde mit dem Zusammenschluss von Einzelinformationen zu komplexen Einheiten erklärt (Chunking), die das System als eine einzige Informationseinheit verarbeitet. Mit Hilfe dieser „funktionalen Kapazitätserhöhung“ eröffneten sich scheinbar viele Möglichkeiten zur 
Steigerung der Behaltensleistung, z.B. über die Bildung von „Superchunks“ in einem hierarchischen „Chunking“-System (vgl. Mandler, 1967).

Baddeley beschäftigte sich in seinem Modell nicht nur mit dem strukturellen Aspekt des Kurzzeitgedächtnisses (Speicherung), sondern ging darüber hinaus auf die Funktion dieses Speichers ein (Baddeley, 1986; Baddeley \& Hitch, 1974). Die Vorstellung eines einheitlichen Kurzzeitgedächtnisses wurde infolge dessen durch einen Arbeitsspeicher mit verschiedenen Subkomponenten ersetzt. Ein „,visuell-räumlicher Notizblock“ (engl. „visuospatial sketchpad“) sollte speziell für die Speicherung visuell-räumlicher Eindrücke und eine „phonologische Schleife“ (engl. ,phonological store“ bzw. „phonological loop“) für die Aufrechterhaltung sprachlich-akustischer Informationen zuständig sein. Dieses Halten von Informationen im Arbeitsspeicher wird möglich, indem Informationen so lange wiederholt in die phonologische Schleife eingespeist werden, bis sich die „Gedächtnisspuren“ verfestigt haben und der Transfer ins Langzeitgedächtnis abgeschlossen ist. Die Kontrolle und Koordination der beiden modalitätsspezifischen Subsysteme obliegt einer modalitätsunabhängigen ,zentralen Exekutive“ (engl. „central executive“). Sie sorgt dafür, dass mit sich stellenden Anforderungen angemessen umgegangen wird, indem beispielsweise die zur Verfügung stehenden Ressourcen gut verteilt werden (vgl. Baddeley, 2000, 2002). Dieses Modell wird in Abbildung 2.5 veranschaulicht.

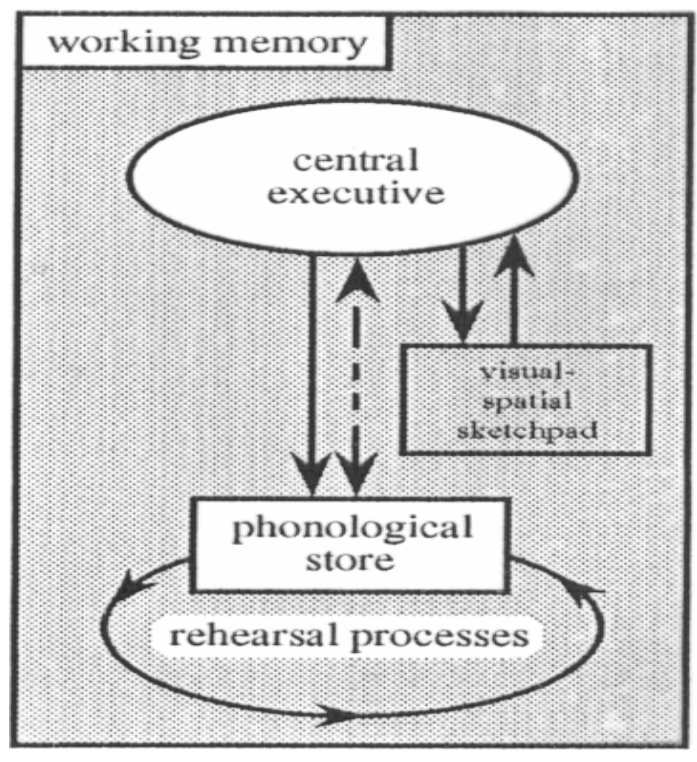

Abbildung 2.5: Das Mehrkomponentenmodell des Arbeitsgedächtnismodells (vgl. Baddeley, 1986)

Für einen effektiven Strategiegebrauch ist es erforderlich, dass genügend Ressourcen für die Bearbeitung einer Aufgabe zur Verfügung stehen. Ansonsten ist zu befürchten, dass die Anforderungen der Aufgabe nicht erkannt werden oder die Qualität des Verhaltens und 
der Erinnerungsleistung deutlich hinter den Erwartungen zurückbleibt. Mit zunehmender Übung und Erfahrung sollte die Kapazitätsbelastung durch den Strategieeinsatz immer geringer werden, da die Anforderungen an der Aufgabenstruktur (z.B. Vorhandensein von Kategorien) schneller erkannt werden (Bjorklund, 1980b) und das Verhalten immer ökonomischer abläuft. Dadurch erklärt sich auch die beobachtete zunehmende Verbesserung strategischer Verhaltensweisen und ihrer Effektivität.

\section{Entwicklungsbefunde}

Zwischen dem 4. und 14. Lebensjahr kann ein deutlicher Anstieg der Informationsverarbeitungsgeschwindigkeit und der Gedächtnisspanne beobachtet werden (vgl. Brown \& Hulme, 1995; Hulme, Thomson, Muir \& Lawrence, 1994). Aufgrund des schnelleren Ablaufs der kognitiven Vorgänge sind ältere Kinder und Erwachsene in der Lage, gleichzeitig eine größere Anzahl an mentalen Operationen im Arbeitsgedächtnis auszuführen (vgl. Hale, 1990; Kail \& Salthouse, 1994; LeBlanc, Muise \& Blanchard, 1992). Ihr Leistungsvorteil gegenüber jüngeren Kindern beruht demnach darauf, dass sie sowohl für die einzelne Aufgabenbearbeitung weniger Ressourcen benötigen als auch insgesamt über ein größeres Ausmaß an freien Ressourcen verfügen, da auch andere Aktivitäten weniger Kapazität in Anspruch nehmen (Siegler, 2001). Bei Anforderungen zum kategorialen Organisieren äußert sich dies darin, dass jüngere Kinder im Vergleich zu älteren Kindern kleinere kategoriale Cluster bilden. Dieses konnte sowohl in Bezug auf die Sortierphase (Lange \& Hultsch, 1970) als auch hinsichtlich der Abrufphase beobachtet werden (Goldman \& Levine, 1963; Lange \& Jackson, 1974; Liberty \& Ornstein, 1973; Worden, 1974).

Die höhere Schnelligkeit, mit der kognitive Anforderungen bearbeitet werden können, wirkt sich auch in Form einer besseren Leistung aus. Case, Kurland und Goldberg (1982) zeigten diesen Zusammenhang für den Umgang mit verbalem Lernmaterial. In ihrer Untersuchung traten keine Altersunterschiede in der Behaltensleistung auf, wenn die Artikulationsrate bei Kindern und Erwachsenen gleich war.

Die Arbeitsgedächtniskapazität sollte demnach in allen Phasen der Strategieproduktion eine Rolle spielen:

- $\quad$ in der Einstiegsphase, in der nach einer Strategie für die Bearbeitung der Aufgabenanforderung gesucht und die Entscheidung für eine solche gefällt werden muss

- $\quad$ in der Initiierungsphase, in der die allgemeinen Überlegungen hinsichtlich der Aufgabenbearbeitung spezifiziert werden müssen 
- $\quad$ bei der Durchführung, bei der die Vorüberlegungen in konkretes Verhalten umgesetzt werden müssen.

Um die Bedeutung der Arbeitsgedächtniskapazität experimentell zu überprüfen, sind grundsätzlich zwei unterschiedliche Vorgehensweisen geeignet: Eine Möglichkeit besteht darin, die Auswirkung der Erhöhung der kognitiven Anforderungen zu untersuchen. Mit Hilfe von sog. Dual-Task-Aufgaben konnte bei unterschiedlichen Strategien gezeigt werden, dass jüngere Kinder in ihrer Leistung beeinträchtigt werden, wenn sie gleichzeitig eine zweite Anforderung erfüllen müssen (z.B. Guttentag, 1984; Miller, Seier, Probert \& Aloise, 1991). Die Auswirkungen auf die Anwendung einer Organisationsstrategie untersuchten Bjorklund und Harnishfeger (1987, Studie 1). Es zeigte sich, dass Erwachsene und Zwölfjährige bei vergleichbarer kognitiver Beanspruchung durch ihren Strategieeinsatz einen höheren Nutzen hatten als Neunjährige, deren Reproduktionsleistung deutlich geringer war. Unter der Standardinstruktion der Dual-Task-Aufgabe lässt sich nur der Einfluss der Arbeitsgedächtniskapazität auf die Durchführung und nicht auf die Initiierung der Strategie untersuchen. Dieser Fragestellung widmeten sich Miller, Seier, Probert und Aloise (1991), indem sie bei ihrer Stichprobe am Anfang den spontanen Einsatz der Selektionsstrategie erhoben und erst später die Dual-Task-Prozedur einführten. Es zeigte sich, dass es bei den jüngeren spontanen Strategen (5- und 6-jährige) eine stärkere Interferenz zwischen der Zusatzanforderung (Finger-Tappen) und der Strategieanwendung gab als bei den 9- und 10jährigen. Die Spezifität der Ressourcenbelastung für bestimmte Arten von Strategien wird daran deutlich, dass eine Kontrollgruppe bei der Durchführung einer einfacheren Strategie durch die Sekundäraufgabe weniger beeinflusst wurde.

Die andere Methode zur Überprüfung des Beitrags, den die Kapazität für die Strategieanwendung und -effizienz leistet, besteht in der Reduktion der kognitiven Belastung. Dieses kann z.B. dadurch erreicht werden, dass der Versuchsleiter dem Kind die Strategieproduktion abnimmt oder die Anwendung einer bestimmten Strategie anleitet (vgl. DeMarieDreblow \& Miller, 1988; Miller, Woody-Ramsey \& Aloise, 1991). Die Anstrengung lässt sich beispielsweise durch die wiederholte Beschäftigung mit entsprechenden Anforderungen reduzieren.

Die altersspezifische Effektivität des strategischen Verhaltens lässt sich auch daran erkennen, dass die Behaltensleistung durch die Nutzung von Ordnungskriterien in sehr unterschiedlichem Ausmaß verbessert wird. $\mathrm{Ab}$ dem 9. Lebensjahr hängt die Reproduktionsleistung mit dem Ausmaß der subjektiven Organisation zusammen (Laurence, 
1966), ab dem 14. Lebensjahr findet sich auch ein Zusammenhang mit dem kategorialen Organisieren (Gerjuoy \& Spitz, 1966). Darüber hinaus spielt auch die Anzahl der verwendeten Kategorien eine wichtige Rolle für die Effektivität der Organisationsstrategie (Mandler \& Stephens, 1967). Während Zweitklässler nur von der Verwendung kleinerer Gruppierungen profitieren, haben ältere Kinder (Viert- und Sechstklässler) auch von größeren Gruppen (6 Items) einen Nutzen (Lange \& Griffith, 1990).

Das Ausmaß frei verfügbarer Ressourcen scheint also dazu zu führen, dass auch komplexe und damit kapazitätsbelastende Strategien mit zunehmendem Alter effektiv eingesetzt werden können und zur Steigerung der Gedächtnisleistung beitragen (vgl. WoodyDorning \& Miller, 2001). Hiervon würden alle Formen von Gruppierungsprozessen profitieren. Die bisher berichteten Analysen haben allerdings gezeigt, dass gerade das Organisieren nach kategorialen Kriterien ein Verhalten ist, das mit zunehmendem Alter immer häufiger bei Kindern beobachtet wird und zur Verbesserung der Erinnerungsleistung beiträgt. Die Frage, warum es genau zu dieser Form der Gruppierungsprozesse kommt, lässt sich unter Berücksichtigung von kognitiven Prozessen auf unterschiedlichen Ebenen beantworten.

Anhänger der Vorwissenshypothese (vgl. Bjorklund, 1985, 1987) führen das Verhalten auf basale kognitive Prozesse zurück. Für den spontanen Einsatz von Strategien werden automatische Aktivierungsprozesse verantwortlich gemacht. Auf der anderen Seite können auch höhere kognitive Prozesse an dem Zustandekommen des Phänomens „kategoriale Organisierungsprozesse“ beteiligt sein. Eine entscheidende Bedeutung kommt dabei den „Überwachungs-, Steuerungs- und Koordinierungsinstanzen“ zu, zu denen das Metagedächtnis, aber auch die Zentrale Exekutive gehört. Während die Zentrale Exekutive unter anderem für einen kontrollierten und selektiven Strategieeinsatz sorgt, ist das Metagedächtnis daran beteiligt, dass dieser bewusst und intentional abläuft. Die zugrunde liegende Annahme ist, dass Organisationsverhalten dann gezeigt wird, wenn das Kind weiß, dass es ihm beim Behalten und Erinnern von Informationen hilft. Sobald ein Verständnis für Lern- und Gedächtnisprozesse vorhanden ist, sollten Hinweisreize erkannt und das eigene Lernverhalten kritisch reflektiert werden. Im Gegensatz zu den unbewussten Einflüssen der allgemeinen Wissensbasis sollte das Wissen über Gedächtnisvorgänge (Metagedächtnis) die aktive und bewusste Interpretation von eingehenden Informationen fördern. 


\subsubsection{Vorwissen}

Wie bereits erwähnt wird die Wissensbasis von verschiedenen Forschern als eine zentrale Determinante beim Einsatz kategorialer Organisationsprozesse angesehen (z.B. Bjorklund, 1988; Chi, 1985). Bei der Beschäftigung mit Wissensaspekten kann es allerdings um sehr unterschiedliche Wissensinhalte gehen. Dazu zählt unter anderem das Wissen über Fakten und konkrete Handlungsweisen, aber auch über komplexe Zusammenhänge.

Für eine Taxonomie der Gedächtnisstruktur hat sich die Unterteilung in ein deklaratives und nicht-deklaratives Gedächtnis bewährt, da sich verschiedene andere Konzeptualisierungen relativ gut darin integrieren lassen.

Das „nicht-deklarative“ (reflexive) Gedächtnis, das von einigen Autoren aufgrund seiner überwiegend unbewussten Inhalte auch als ,implizites“ Gedächtnis bezeichnet wird, ist langsamer und weniger flexibel, aber dafür zuverlässiger. Neben einem prozeduralen Gedächtnis, in dem Informationen zur Durchführung einfacher kognitiver, motorischer oder wahrnehmungsbezogener Fertigkeiten abgespeichert werden, sind dort auch „Priming“- und Konditionierungsprozesse lokalisiert.

Das deklarative Gedächtnis gliedert sich wiederum in das episodische und das semantische Gedächtnis auf (vgl. Tulving, 1972, 1982, 2000). Während im episodischen Gedächtnis erfahrungsbasierte Informationen unter Berücksichtigung der zeitlichen Sequenz verarbeitet und gespeichert werden, enthält das semantische Gedächtnis das allgemeine Wissen (z.B. über Sprache, Regeln und Konzepte). Die Wissenseinheiten sind dabei konzeptuell verbunden und können bewusst erinnert werden. Während allerdings die Informationsverarbeitung im episodischen Gedächtnis bewusst abläuft und als anstrengend erlebt wird, erfolgt der Informationsabruf aus dem semantischen Gedächtnis weitgehend automatisch. Kennzeichnend für das deklarative Gedächtnis ist seine hohe Prozessgeschwindigkeit und Flexibilität sowie seine Anfälligkeit gegenüber Störeinflüssen (z.B. Abrufschwierigkeiten).

Bei der Abspeicherung und dem Abruf von Wissen geht es aber nicht nur darum, was für Informationen abgespeichert sind, sondern auch darum, wie diese Informationen repräsentiert sind. Es gibt unterschiedliche Vorstellungen darüber, auf welche Art die Organisation semantischer Information im Gedächtnis in den kognitiven Strukturen abgebildet wird. Günstig ist eine graphische Abbildung als semantisches Netzwerk. Quillian (1968) stellte ein Modell für die Organisation sprachlicher Inhalte im Gedächtnis auf, in dem 
begriffliches Wissen als Lexikon in Form eines nicht-hierarchischen Netzes abgespeichert sein sollte (Netzwerkmodell). Begriffe bilden in diesem Modell die „Knoten“, wobei Namen (,type“) und die Bedeutung der Begriffe ("token“) besondere Knoten darstellen. Zwischen diesen Knoten bestehen spezielle Verknüpfungen, an denen entlang sich die Aktivierung ausbreitet (Collins \& Loftus, 1975). Bei den Verknüpfungen muss es sich allerdings nicht nur um assoziative Relationen handeln. Die Orientierung an anderen Kriterien kann zu einer Vielzahl unterschiedlicher Verknüpfungen führen (z.B. Klasseninklusionsrelationen, Merkmalsrelationen, thematische Relationen). Der Stellenwert einer Verknüpfung zeigt sich an ihrer jeweiligen Stärke. Ob Wissensbestandteile einzeln oder zusammen erinnert werden hängt folglich von der Anzahl der vorhandenen Knoten und der Stärke ihrer Verknüpfungen $a b$.

In der Entwicklungspsychologie wurde den Repräsentationsstrukturen des konzeptuellen Wissens lange Zeit eine große Bedeutung beigemessen, da von spezifischen qualitativen Entwicklungsveränderungen ausgegangen wurde. Für die erste Hälfte der Grundschulzeit wurde ein Wechsel von einer syntagmatischen, perzeptuellen und „funktionalthematischen“ Repräsentationsform hin zu einer eher paradigmatischen, konzeptuellen und taxonomisch-hierarchischen“ Repräsentation postuliert (z.B. Brown \& Berko, 1960; Ervin, 1961; Melkman \& Deutsch, 1977; Melkman, Tversky \& Baratz, 1981; Worden, 1976). Dennoch bleibt es fraglich, ob eine taxonomische Wissensstruktur wirklich ein Indiz für eine reifere Art der Wissensrepräsentation im Vergleich $\mathrm{zu}$ funktionalen oder perzeptuellen Wissensstrukturen ist. Möglicherweise spiegeln die unterschiedlichen Wissensrepräsentationen mit den jeweiligen Relationen die unterschiedlichen Sichtweisen der Welt von Kindern in unterschiedlichen Altersstufen wieder, die alle für sich genommen „,angemessen“ sein können (vgl. Markman \& Callanan, 1984). Auch funktional-thematische Relationen können sehr nützlich sein und werden auch von älteren Kindern genutzt (vgl. Davis, 1976). Gleichzeitig können auch jüngere Kinder taxonomische Relationen erkennen und nutzen (vgl. Bjorklund, 1980a; Fenson, Vella \& Kennedy, 1989; Gelman \& Baillargeon, 1983; Mandler \& Bauer, 1988; Mandler, Fivush \& Reznick, 1987; Markman \& Callanan, 1984; Markman, Cox \& Machida, 1981; Scott, Sercuk \& Mundy, 1982; Siaw, 1984; Smiley \& Brown, 1979). Siebenjährige verstehen sogar komplexe taxonomische Kategorien mit vier Hierarchie-Ebenen (Greene, 1989). Alternativ kann diskutiert werden, ob die Bevorzugung taxonomischer gegenüber funktionalen Kategorisierungskriterien wirklich mit Veränderungen der Wissensrepräsentation einhergehen (Lange, 1978; Markman, 1989, Kapitel 2; Schneider 
\& Pressley, 1989, S. 55ff.). Zwischen dem 6. und 11. Lebensjahr scheinen funktionalthematische und taxonomisch-hierarchische Relationen parallel zueinander vorzuliegen.

Altersunterschiede zeigen sich nur in der Präferenz für bestimmte Ordnungskriterien.

Auch hinsichtlich kategorialen Wissens existieren von früher Kindheit an verschiedene Repräsentationsformen (enaktiv, bildhaft, sprachlich-symbolisch) nebeneinander. Prinzipielle Entwicklungsveränderungen in der Präsentation semantischer Information sind eher unwahrscheinlich (Glass, Holyoak \& Kossan, 1977; Kluwe, Wolke \& Bunge, 1982; Krist \& Wilkening, 1991; Mansfield, 1977; McFarland \& Kellas, 1975). Spätestens ab dem 6. Lebensjahr stimmen die Repräsentationsstrukturen kategorialen Wissens mit der von Erwachsenen überein (Glass et al., 1977; Mansfield, 1977; Rosner \& Hayes, 1977). Nur das Ausmaß kategorialen Wissens (= die Anzahl der Knoten und Stärke der Relationen) scheint Entwicklungsveränderungen zu unterliegen.

\section{Bedeutung für den Einsatz kategorialer Organisationsstrategien}

Ein umfangreiches bereichsspezifisches Wissen erleichtert durch die reichhaltige Repräsentation der Wissensbestandteile den Zugriff auf bestimmte Informationen (z.B. Lindberg, 1980). Durch die Aktivierung von Verknüpfungen zwischen Items können Lerninhalte automatisch zusammen erinnert werden (Bjorklund \& Bjorklund, 1985). Darüber hinaus kann sich eine gute Wissensbasis positiv auf das Erkennen und den Einsatz von Strategien auswirken (Bjorklund, 1987; Hasselhorn, 1995). Ein Mindestmaß an Wissen über das Lernmaterial ist eine Grundbedingung dafür, dass von den Kindern überhaupt Beziehungen zwischen einzelnen Objekten hergestellt werden. Die Zusammenhänge werden für die Kinder umso offensichtlicher, je umfangreicher das Wissen über einen bestimmten Bereich ist und je vielfältiger die Verknüpfungen zwischen den Wissensbestandteilen sind (vgl. Ornstein \& Naus, 1985). Dementsprechend werden neue Strategien von Kindern am ehesten unter vertrauten Bedingungen bzw. bei bekannten Inhalten gezeigt (Chi, 1981).

Das Vorliegen einer starken Verknüpfung zwischen verschiedenen Items kann sich darin äußern, dass diese in der Abrufphase kurz nacheinander genannt werden. Generell sollten die Wiedergabezeiten bei hoch-assoziativem Lernmaterial geringer sein als bei niedrig-assoziativem (Bjorklund \& Jacobs, 1985). Bei kategorisierbarem Lernmaterial sollten die Interitemlatenzen kürzer sein, wenn es sich um Exemplare derselben Kategorie handelt (vgl. Pollio, Richards \& Lucas, 1969), als wenn die Objekte aus unterschiedlichen Kategorien stammen (vgl. Howard \& Kahana, 2001). Die höhere Zeit kommt dadurch zustande, dass für den Abruf von Items aus einer neuen Kategorie vorher der Zugriff auf die alte Kategorie 
beendet und die neue Kategorie aufgerufen werden muss (vgl. Patterson, Meltzer \& Mandler, 1971).

Die Beobachtung, dass sich bei Dritt- und Fünftklässlern die interkategorialen Latenzen nicht von den intrakategorialen Latenzen bei niedrig-assoziativem Material unterschied, wurde von Bjorklund und Jacobs (1985) als Beleg dafür gedeutet, dass in dieser Altersstufe Kategorisierungsprozesse automatisch ablaufen.

\section{Bedeutung für einen effektiven Strategiegebrauch}

Eine umfangreiche Wissensbasis kann sich infolge der oben beschriebenen Faktoren sehr günstig auf die Behaltensleistung auswirken (vgl. Bjorklund, 2000; Kee \& Davis, 1988, 1990; Körkel, 1987). Für die Untersuchung der Bedeutung des Wissens für eine effektive Strategieanwendung gibt es grundsätzlich zwei Möglichkeiten. Einmal kann die Aufgabe in einen bedeutungshaltigen Kontext eingebettet werden (vgl. Miller, Seier, Barron \& Probert, 1994; Woody-Ramsey \& Miller, 1988). Für die Untersuchung des Einflusses auf den Nutzen der Organisationsstrategie scheint eine andere Herangehensweisen geeigneter zu sein. Dabei werden bei den zu kategorisierenden Items wissensrelevante Aspekte manipuliert, indem z.B. die kategoriale Typizität des Lernmaterials verändert wird. Es kann davon ausgegangen werden, dass es einfacher ist, Items zu einer Kategorie zusammenzufügen, wenn sie für diese sehr typisch sind (z.B. Hund und Katze für Tiere) als wenn sie eher untypisch sind (z.B. Anemone, Seestern für Tiere). Dieses betrifft sowohl das Erkennen kategorialer Beziehungen zwischen Items als auch den Zugriff auf diese kategorialen Strukturen (vgl. Bjorklund, MuirBroaddus \& Schneider, 1990). Die Darbietung von hochtypischen Items führt auch mit einer höheren Wahrscheinlichkeit dazu, dass der Einsatz einer kategorialen Organisationsstrategie effektiv ist (Bjorklund, 1988; Bjorklund, Schneider, Cassel \& Ashley, 1994).

Auch bereichsspezifisches Fachwissen hat einen positiven Einfluss auf die Strategieeffektivität. Am Beispiel von Schachkenntnissen konnte Chi (1978) demonstrieren, dass zehnjährige Experten sich eine Spielstellung schneller einprägen und besser rekonstruieren konnten als erwachsene Schachanfänger. Diese Vorteile verschwanden, wenn den Spielstellungen die inhaltliche Grundlage entzogen wurde, d.h. die Muster keinen Sinn mehr ergaben. Der infolge einer umfassenden Wissensbasis erzielte Vorteil ist folglich sowohl auf quantitative als auch auf qualitative Unterschiede in den Repräsentationsstrukturen zurückführbar (vgl. Chi, Hutchinson \& Robin, 1989; Lindberg, 1991; Schneider, Gruber, Gold \& Opwis, 1993). 
Der Einfluss auf die Strategie des kategorialen Organisierens ließ sich am Beispiel von Fußballkenntnissen demonstrieren. Während bei Neunjährigen Experten ein bedeutsamer Zusammenhang zwischen der Organisation nach Kategorien in der Lern- und Abrufphase und der Behaltensleistung bestand, gab es bei sieben- und neunjährigen Anfängern diesen Zusammenhang nicht (Schneider \& Bjorklund, 1992). Auf der anderen Seite gibt es auch Befunde, die zeigen, dass Expertise zwar das Textgedächtnis und Textverständnis positiv beeinflusst (z.B. Schneider, Körkel \& Weinert, 1989), aber nicht die Leistungen in strategischen Gedächtnisaufgaben wie bei Sort-Recall-Anforderungen (Schneider, Bjorklund \& Maier-Brückner, 1996).

\subsubsection{Metagedächtnis}

Als weiterer kognitiver Faktor, der einen Einfluss auf die Möglichkeit einer effektiven Strategienutzung hat, soll nun nach dem Arbeitsgedächtnis und der Wissensbasis das Metagedächtnis betrachtet werden. Das Metagedächtnis kann in gewisser Hinsicht als Spezialfall des Wissens einer Person angesehen werden, da es ihr „Wissen über Wissen“ (Tulving \& Madigan, 1970, S. 477) oder ihren „Metaplan“ (Miller et al., 1960, S. 129) abbildet. Metakognitiven Prozessen wird für eine Vielzahl von kognitiven Funktionen (Aufmerksamkeit, Gedächtnis, Kommunikation, Problemlösen, Intelligenz, etc.) eine große Bedeutung beigemessen (vgl. Moses \& Baird, 1999; Kuhn, 2000; Wimmer \& Thornquist, 1980). Dieser Einfluss kann in Abhängigkeit von individuellen Voraussetzungen der Personen (z.B. Intelligenz) und kulturellen Besonderheiten variieren (vgl. Kurtz \& Weinert, 1989; Kurtz, Borkowski \& Deshmukh, 1988). Die Herangehensweise an dieses Thema von verschiedenen Forschungsrichtungen mit unterschiedlichen Fragestellungen hat zu einer großen Heterogenität und Verschwommenheit dieses Konzeptes geführt (vgl. Flavell, 1981; Schneider \& Pressley, 1997). Die vorhandenen Differenzen hinsichtlich der Einordnung dieses Konstruktes (vgl. Nelson \& Narens, 1994) oder in Bezug auf Definitionskriterien, wie die Einbeziehung unbewusster Prozesse (z.B. Cornoldi, 1998; Koriat \& Goldsmith, 1998) oder deren Ausschluss (z.B. Hacker, 1998) haben aber nie dazu geführt, dass die Existenz oder Bedeutung des Metagedächtnisses in Frage gestellt wurde, sondern lediglich aufgezeigt, dass seine Erforschung noch lange nicht abgeschlossen ist (vgl. Koriat, 2000; Metcalfe, 2000).

Insgesamt lässt sich bei den meisten Forschern eine deklarative Komponente, die Faktenwissen über Gedächtnisprozesse und -inhalte beinhaltet, von nicht-deklarativen 
Facetten, die sich stärker auf Handlungsaspekte beziehen, abgrenzen. Diese Unterscheidung scheint für eine übergreifende Auseinandersetzung mit dieser Thematik geeigneter zu sein als die individuelle metakognitive Konzeptualisierung einer Aufgabe (vgl. Cornoldi, 1998).

Das deklarative Metagedächtnis umfasst Wissen über den Einfluss spezieller Variablen, wie der Person, der Aufgaben und von Strategien (Cavenaugh \& Perlmutter, 1982). Dabei kann systemisches Wissen, d.h. Wissen über das kognitive System, Wirkmechanismen, Kontextbedingungen und Strategien, von epistemischem Wissen, das sich auf die eigene Person bezieht, abgegrenzt werden (Hasselhorn, 2001). Das systemische Wissen oder generelle Strategiewissen (Borkowski, 1996) lässt sich darüber hinaus in spezifisches und relationales Strategiewissen aufgliedern. Paris und seine Kollegen führten darüber hinaus die Komponente des ,metakognitiven Bedingungswissens“ ein (Paris \& Lindauer, 1982; Paris \& Oka, 1986), mit der die Fähigkeit zur Bewertung oder Erklärung der getroffenen Entscheidungen bezüglich ihres Lernverhaltens erfasst wird.

Dem gegenüber steht das nicht-deklarative Metagedächtnis, hinter dem sich verschiedene Konstrukte verbergen, die sich nicht immer klar voneinander abgrenzen lassen. Das Wissen darüber, wann eine spezifische Gedächtnisaktivität eingesetzt werden muss, wird als Sensitivität oder prozedurales Metagedächtnis bezeichnet. Die Sensitivitätskategorie beinhaltet sowohl Erfahrungswissen als auch intuitive Aspekte (Flavell, 1978; Hasselhorn, 1992). Mit diesen Prozessen sind immer auch metakognitive Erfahrungen im Sinne von bewussten kognitiven Empfindungen oder affektiven Zuständen verbunden. Diese werden z.T. als eigenständige Kategorie betrachtet (Hasselhorn, 1992) oder unter allgemeine Wissensaspekte subsummiert (Flavell, 1978). Das prozedurale Metagedächtnis umfasst metakognitive Kontrollprozesse (exekutive Metakognitionen), die von der Planung, Koordination und Steuerung von Handlungsabläufen bis zur Korrektur kognitiver Prozesse reichen (Brown, Bransford, Ferrara \& Campione, 1983). Bei diesen exekutiven Prozessen können, in Abhängigkeit von der Richtung der Informationsweiterleitung, zentral gesteuerte Selbstüberwachungsprozesse (self-monitoring) für die Planung, Anweisung und Durchführung von Aktivitäten von am aktuellen Geschehen orientierten Selbstregulationsmechanismen (self-regulation) unterschieden werden (Borkowski, 1996; Nelson \& Narens, 1990).

Aufgrund der Funktionsvielfalt des Konstruktes und des daraus resultierenden komplexen Wirkungsgeflechts erweist sich die Entwicklung einer konkreten vollständigen Modellvorstellung für „exekutive Metakognitionen“ bzw. „metakognitive Exekutivfunktionen“ als ebenso schwierig wie die Integration in bereits bestehende Modelle. 
Während einige Forscher Metakognitionen als Teil der Wissensbasis betrachten (Tulving \& Madigan, 1970; Miller et al., 1960), spricht Baddeley (2000, 2002) die beschriebenen Funktionen in seinem Arbeitsgedächtnismodell einem modalitätsunabhängigen System, der zentralen Exekutive, zu. Diesen Zusammenhang zwischen Metakognitionen und exekutiven Kontrollprozessen zeigen auch aktuelle Neuroimaging-Studien, in denen auf die Beteiligung verschiedener Areale im frontalen Cortex an diesen Prozessen hingewiesen wird (FernandezDuque, Baird \& Posner, 2000; Shimamura, 2000). Auch deklarative und prozedurale Aspekte des Metagedächtnisses sind eng miteinander verflochten und beeinflussen sich wechselseitig. Die Reflektion über das eigene Verhalten kann zur Entstehung von neuem Wissen über die eigenen Kompetenzen und allgemeinen Lernprozesse beitragen. Dieses Wissen über die eigenen Fähigkeiten kann wiederum zu einer vermehrten Kontrolle des eigenen Verhaltens führen.

Die bisherigen Bemühungen, das sehr heterogene Konzept „Metakognition“ von anderen Konzepten klar abzugrenzen ohne wesentliche metakognitive Aspekte auszulassen, haben zu einer Vielzahl alternativer Klassifikationen metakognitiver Komponenten geführt (Brown, 1978; Cavanaugh, 1989; Flavell \& Wellman, 1977; Wellman, 1983). Hasselhorn (1992) versuchte, diese unterschiedlichen Vorstellungen in einem integrativen Klassifikationsschema zu bündeln, in dem die fünf wichtigsten Subkategorien der Metakognition enthalten sind:

\section{Systemisches Wissen}

a) Wissen über das eigene kognitive System und seine Funktionsgesetze

b) Wissen über Lernanforderungen

c) Wissen über Strategien

\section{Epistemisches Wissen}

a) Wissen über eigene aktuelle Gedächtniszustände bzw. Lernbereitschaften

b) Wissen über die Inhalte und Grenzen eigenen Wissens

c)Wissen über die Verwendungsmöglichkeiten eigenen Wissens

\section{Exekutive Prozesse (Kontrolle)}

a) Planung eigener Lernprozesse

b) Überwachung eigener Lernprozesse

c) Steuerung eigener Lernprozesse 


\section{Sensitivität für die Möglichkeiten kognitiver Aktivitäten}

a) Erfahrungswissen

b) Intuition

\section{Metakognitive Erfahrungen bezüglich der eigenen kognitiven Aktivität}

a) bewusste kognitive Empfindungen

b) bewusste affektive Zustände

Im Rahmen einer angestrebten empirischen Erfassung von Metakognitionen lässt sich diese Komplexität allerdings nicht abbilden, sondern erfordert die Fokussierung auf einzelne Aspekte. Im Folgenden soll daher der Schwerpunkt auf der Erhebung des aufgabenspezifischen Strategiewissens liegen, d.h. der Kenntnisse in Bezug auf die Strategie des kategorialen Organisierens.

\section{Empirische Erfassung des metakognitiven Strategiewissens}

Die empirische Erfassung und Deutung des metakognitiven Wissens bergen verschiedenen Schwierigkeiten in sich. So wies Reyna (1996) zu Recht auf die Möglichkeit hin, dass die Beobachtung, dass von Kindern zwischen Strategiegebrauch und Gedächtnisleistung kein Zusammenhang gesehen wird, nicht zwangsläufig darauf beruhen muss, dass ihr Metagedächtnis unzureichend entwickelt ist. Es könnte sich auch um eine realistische Einschätzung der Zusammenhänge handeln, wenn z.B jüngere Kinder erkennen, dass sie in den meisten Fällen nicht von strategischen Verhaltensweisen profitieren. Das veränderte Strategiewissen bei älteren Kindern würde in diesem Fall damit zusammenhängen, dass ältere Kinder (im Gegensatz zu jüngeren) wirklich von einer Strategie profitieren.

Eine andere Problematik könnte damit zusammenhängen, dass Kinder ihr Strategiewissen auf besondere Art und Weise abgespeichert haben, was in der konkreten Situation nicht unbedingt erkennbar ist. Möglicherweise verfügen sie über abstrakte metakognitive Kenntnisse, die für sie aber keine Handlungsrelevanz besitzen. Die Bedeutung des Metagedächtnisses für die Bewältigung der gestellten Anforderung hängt demnach auch von den experimentellen Rahemenbedingungen ab. Nach Ansicht von Reyna (1996) wird die Bedeutung des metakognitiven Wissens bereits dadurch eingeschränkt, dass bei der Untersuchung von spontanem Strategiegebrauch in der Regel auf Fragen verzichtet wird, die als Auslöser fungieren können und damit vorhandenes Wissen ins Bewusstsein treten lassen. 
Darüber hinaus dürfen auch verbale Kompetenzen nicht vernachlässigt werden, die die Kinder mehr oder weniger gut in die Lage versetzen, ihr Wissen transparent zu machen. Bevor die vorhandene Einsicht verbal artikuliert werden kann, könnten im Verhalten der Kinder bereits Anzeichen für ein wachsendes Verständnis für Gedächtnisprozesse und Einflussmöglichkeiten erkennbar sein (vgl. Reyna, 1996).

Umgekehrt kann es aber auch zu einer Überschätzung des Entwicklungsstandes des Metagedächtnisses kommen. Dieses wäre beispielsweise der Fall, wenn Kinder bei der Beurteilung von Gedächtnisstrategien nicht ihre eigene Sichtweise wiedergeben, sondern die expliziten Theorien von Erwachsenen über Gedächtnisphänomene wiederholen. In diesem Fall wäre keine Einsicht in die Leistungsdienlichkeit des Strategiegebrauchs vorhanden. Trotz dieser Einschränkungen - oder gerade deswegen - lohnt es sich, sich mit den Möglichkeiten der Erfassung des Metagedächtnisses auseinanderzusetzen.

Zur Quantifizierung des deklarativen Metagedächtnisses (systemisches und epistemisches Wissen) wurden bisher vor allem Fragebögen und Interviews eingesetzt. Die meisten Verfahren boten allerdings reichlich Anlass zur Kritik (vgl. Hasselhorn, 1994). Aufgrund der bereits beschriebenen Heterogenität des Konstruktes und der ungünstigen methodischen Herangehensweise (z.B. Salatas \& Flavell, 1976) überrascht es nicht, dass bei vielen Messinstrumenten die Gütekriterien nur mittelmäßig ausfielen (vgl. Schlagmüller, Visé \& Schneider, 2001). Als weitgehend reliabel und valide erwiesen hatte sich die Würzburger Testbatterie zum deklarativen Metagedächtnis (Schlagmüller et al., 2001; Schneider, Schlagmüller \& Visé, 1998). Diese setzt sich aus drei Subskalen zu den Bereichen allgemeines deklaratives MG (ADM), Wissen über semantische Kategorisierungsstrategien (SKS) und textverarbeitungsbezogenes MG (TV) zusammen, die sich als relativ unabhängig voneinander erwiesen hatten.

Der Zusammenhang zwischen den Leistungsmerkmalen und dem allgemeinen deklarativen Metagedächtnis hatte sich bisher als nicht besonders aussagekräftig herausgestellt (z.B. Joyner \& Kurtz-Costes, 1997; Schneider, 1985). Im Mittelpunkt des Interesses steht daher die Subskala zum Wissen über semantische Kategorisierungsstrategien, die in den Vorläuferstudien substantiell mit Verhaltensmaßen und Leistungsresultaten korrelierte und eine gute bis befriedigende interne Konsistenz aufwies. Die Items bestanden jeweils aus drei Antwortalternativen, deren Güte die Kinder durch die Vergabe von Noten oder Rangplätzen bewerten sollten. 
Eine andere Möglichkeit, spezifische Metagedächtnisaspekte zu erfassen, bietet die deutsche Übersetzung des Metagedächtnistests von Belmont und Borkowski (1988). Dieser erfasste unter anderem das Verständnisses der Lernerleichterung durch kategoriale Ordnungsstrukturen (Subtest 1: „Organized List“").

Als problematisch bei der Erfassung metakognitiver Aspekte hatte sich allerdings die oft vorhandene Konfundierung mit motivationalen Faktoren erwiesen (vgl. Hasselhorn, Hager \& Baving, 1989). Darüber hinaus konnten auch Messinstrumente mit insgesamt befriedigenden Reliabilitäten (Belmont \& Borkowski, 1988) bei der Untersuchung von jüngeren Kindern nicht zufriedenstellend eingesetzt werden (z.B. Geary, Klostermann \& Adrales, 1989) und lieferten Hinweise darauf, dass in verschiedenen Subtests voneinander unabhängige Aspekte des Metagedächtnisses repräsentiert sind (Hasselhorn, 1994). Neuere Fragebögen, in denen neben dem allgemeinen deklarativen Metagedächtnis auch das textverarbeitungsbezogene und strategiespezifische Wissen erhoben wurde, scheinen das deklarative Metagedächtnis hinreichend reliabel und valide erfassen zu können (Schlagmüller, Visé \& Schneider, 2001). Bei diesen Reliabilitätsanalysen schnitt die Subskala zum strategiespezifischen Metagedächtnis, die in erster Linie das Wissen über semantische Kategorisierungsstrategien erfasste, am besten ab (vgl. Hasselhorn, 1994; Schlagmüller, Visé \& Schneider, 2001).

\section{Entwicklungsbefunde}

Aufgrund der zunehmenden Auseinandersetzung mit Lern- und Gedächtnisanforderungen ist davon auszugehen, dass sich im Laufe der Entwicklung auch das Wissen über Aspekte des Lernens und Gedächtnisprozesse sowohl quantitativ als auch qualitativ verbessert (vgl. Schneider, 1999). Während im Vorschulalter das Wissen über Aufgaben- und Strategievariablen nur rudimentär ist, kommt es während der Grundschulzeit zu einer starken Verbesserung des deklarativen Wissens (Kurtz \& Borkowski, 1987). Dieses zeigt sich sowohl in dem zunehmenden Verständnis für Abrufhinweise (Beal, 1995; Schneider \& Sodian, 1988) und in der stärkeren Präferenz für die Wiedergabe des Kerns einer Aussage im Vergleich zu der wörtlichen Wiedergabe (Kreutzner Leonard \& Flavell, 1975) als auch in der Bevorzugung von taxonomisch gegliedertem gegenüber ungeordnetem Lernmaterial (Schneider \& Sodian, 1988). Zwischen dem 8. und 10. Lebensjahr kann ein Entwicklungsschub hinsichtlich der Entwicklung des deklarativen Metagedächtnisses beobachtet werden (Hasselhorn, Mähler \& Grube, 1995; Justice, 1985; 1986; Kreutzer et al., 1975; Schneider, 1986; Schneider, Körkel \& Vogel, 1987). Am Ende der Grundschulzeit ist 
den meisten Kindern die Bedeutung der Organisationsstrategien für eine erfolgreiche Wiedergabe bewusst. Obwohl bis zum 12. Lebensjahr ein umfangreiches deklaratives metakognitives Wissen erworben wird, ist diese Entwicklung damit nicht abgeschlossen, wie Defizite im Erwachsenenalter zeigen (Schneider \& Pressley, 1997). Hasselhorn (1996) grenzte diesen Zeitraum sogar noch enger auf die Zeit zwischen dem 9. und 10. Lebensjahr ein.

In Bezug auf Fähigkeiten zur Selbstüberwachung zeigt sich ebenfalls eine Abhängigkeit der Genauigkeit der Selbsteinschätzung vom Alter der Kinder und von Kontextvariablen (Bekanntheitsgrad der Aufgabe, Instruktion, Erhebungsmethode), unabhängig davon, ob das Urteil vor der Aufgabenbearbeitung abgegeben werden sollte (Cunningham \& Weaver, 1989) oder im Anschluss (Bisanz, Vesonder \& Voss, 1978; Pressley, Levin, Ghatala \& Ahmad, 1987). Aufgabencharakteristika (Struktur, Bedeutungsgehalt der Items) wirken sich auch auf die subjektive Wiedererkennungswahrscheinlichkeit („Feeling-of-knowing accuracy“) aus (Cultice, Somerville \& Wellman, 1983; DeLoache \& Brown, 1984; Posnansky, 1978; Wellman, 1977).

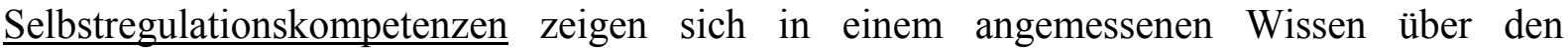
Schwierigkeitsgrad bei der Wiedergabe (Flavell et al., 1970) und einer guten Verteilung der zur Verfügung stehenden Lernzeit (Masur, McIntyre \& Flavell, 1973). Dabei konnte gezeigt werden, dass junge Kinder zwar zwischen den Items diskriminieren, aber diese Information nicht bei einem erneuten Lerndurchgang nutzen (Bisanz et al., 1978; Dufresne \& Kobasigawa, 1989). Spontane aufgabenbezogene Selbstregulationsstrategien waren hingegen auch bei älteren Kindern nur selten zu beobachten, sofern die Aufgabe ein angemessenes Schwierigkeitsniveau besaß (Dufresne \& Kobasigawa, 1989; Leal, Crays \& Moely, 1985), konnten aber trainiert werden (Ghatala, Levin, Pressley \& Goodwin, 1986; Paris \& Newman \& McVey, 1982). Eine hohe Flexibilität der Überwachungsprozesse und der Koordination der Pläne, wie sie bei einem gut entwickelten prozeduralen Metagedächtnis zu erwarten ist, wird aber selten vor der 6. Klasse erreicht (Schneider, 1998).

\section{Die Bedeutung des Metagedächtnisses für den Strategiegebrauch}

Im Allgemeinen wird davon ausgegangen, dass zwischen dem metakognitiven Wissen und dem strategischen Umgang mit Gedächtnisanforderungen ein Zusammenhang besteht (vgl. Borkowski, Milsted \& Hale, 1988; Borkowski \& Turner, 1990). Dem Wissen über Gedächtnisstrategien wird eine bedeutsame Rolle für den Erwerb von Strategien und ihrer Anwendung zugesprochen (Hasselhorn, 1996; Pressley, Borkowski \& O`Sullivan, 1985; 
Schneider, 1999). Umgekehrt kann allerdings die Anwendung von Strategien zur Ausbildung eines guten Metagedächtnisses beitragen.

Unter der Annahme, dass der Strategiegebrauch zu metakognitiven Erkenntnissen bzw. der Ausbildung eines distalen Metagedächtnisses führt, was sich im weiteren Verlauf positiv auf den Strategieerwerb auswirken kann, wurden früher die beobachteten Produktionsdefizite bei jüngeren Schulkindern auf das vermeintlich nicht verfügbare aufgabenspezifische metakognitive Wissen in dieser Situation zurückgeführt (vgl. Flavell, Miller \& Miller, 1993; Schneider \& Pressley, 1997). Im späteren Entwicklungsverlauf wurde eine „bi-direktionale“ Beziehung zwischen Strategieanwendung und metakognitivem Wissen unterstellt: Die erste Anwendung der Organisationsstrategie sollte mit dem Erwerb von neuem aufgabenspezifischen Wissen einhergehen, das wiederum den künftigen Strategiegebrauch erleichtert (vgl. Pressley, Borkowski \& Schneider, 1989).

Die andere Annahme, dass ein distales Metagedächtnis Voraussetzung dafür ist, dass Strategien erkannt und eingesetzt werden, wurde unter anderem durch die Befunde der mikrogenetischen Studie von Schlagmüller und Schneider (2002) untermauert. Diese lieferte Hinweise darauf, dass deklaratives metakognitives Wissen über Organisationskenntnisse sich früher als die tatsächliche Strategieanwendung entwickelt. Die Strategieentdecker verfügten bereits sechs Monate vor ihrem ersten Strategieeinsatz über ein beachtliches Wissen bezüglich der Vorteile von Organisationsstrategien. Anscheinend mussten die Kinder bevor sie die Strategie zum ersten Mal ausprobierten nicht nur Kenntnisse über die allgemeine Nützlichkeit von Strategien haben, sondern mussten davon überzeugt sein, dass der Strategieeinsatz ihnen wirklich helfen würde. Ein ähnliches Entwicklungsmuster konnte aber auch für die Kinder der nicht-strategischen Gruppe beobachtet werden. Die Nicht-Strategen zeigten bereits im Vorfeld der Untersuchung eine Verbesserung ihres metakognitiven Wissens. Die Verbesserungen des metakognitives Wissens im Verlauf der Studie entsprachen in etwa dem Niveau der strategischen Kinder. In einer „Follow-up“-Untersuchung konnte gezeigt werden, dass die Kinder, die in der mikrogenetischen Studie als Nicht-Strategen klassifiziert worden waren, ein paar Monate später die Strategie erwarben (Schlagmüller \& Schneider, 2002). Diese Befunde stehen in Einklang mit der Strategie-Emergenz-Theorie (Hasselhorn, 1996), die annimmt, dass die im Verlauf der Grundschuljahre beobachtbaren qualitativen Veränderungen der strategischen Kontrollprozesse in erster Linie das Ergebnis von einem verbesserten spezifischen Metagedächtnis sind. Auch der postulierte Zeitraum für den Erwerb eines angemessenen Metagedächtnisses (zwischen dem 8. und 10. Lebensjahr) wird durch die Daten von Schlagmüller und Schneider (2002) gestützt. 
Das metakognitive Wissen sollte sich allerdings nicht nur allgemein auf den Strategiegebrauch auswirken, sondern auch in differenzieller Hinsicht von Bedeutung sein. Konkret ist davon auszugehen, dass die Qualität des distalen Metagedächtnisses sowohl für den erstmaligen Einsatz einer Strategie als auch für das Aufrechterhalten einer Strategie von Bedeutung ist. Sodian und Schneider (1999) führten die geringe Stabilität des strategischen Verhaltens, wie andere Autoren vorher (z.B. Bjorklund \& Jacobs, 1985; Hasselhorn, 1992; Rabinowitz, 1984), auf die möglicherweise fehlende Einsicht in die relevanten Abläufe zurück. Das beobachtete Verhalten wäre unter diesen Voraussetzungen nur dadurch zustande gekommen, dass aufgrund der assoziativen Verbindungen zwischen den Items bei den Kindern ein Gefühl des „Zusammenpassens“ entstanden ist und sie dadurch zusammengelegt wurden. Dieses würde implizieren, dass die Strategie sehr störanfällig und fragil ist und besonders bei leichten Veränderungen der Aufgabe (unterschiedliche Items, unterschiedliche Darbietungsmodalität, etc.) zum Verlust der Strategie führt.

Anhand der Ergebnisse eines aufgabenbezogenen Metagedächtnis-Interviews wurde deutlich, dass die meisten Vorschüler die Organisation nach Farbe für die beste Strategie hielten, obwohl niemand von ihnen diese Strategie bei der eigenen Aufgabenbearbeitung nutzte. Für das konzeptuelle Organisieren als beste Strategie entschied sich ein Drittel der Sechsjährigen, und die große Mehrheit der älteren Kinder, die meistens außerdem gedächtnisbezogene Erklärungen für ihre Urteile liefern konnten. Da 32\% der Kinder mit hohen Sortierwerten im Alter von 4 oder 6 Jahren die Strategie zu späteren Messzeitpunkten aufrechterhielten, wurde die Prädiktorfunktion des Metagedächtnisses für die Wiederentdeckung der Strategie näher untersucht. Leider konnte dieser Zusammenhang zur Stabilität der Strategieanwendung nicht eindeutig nachgewiesen werden. Interpretiert wurde dieses Ergebnis dahingehend, dass die Anforderung an das Verständnis der Kinder, die Vorteile der konzeptuellen Organisation gegenüber farblicher Organisation zu sehen, zu spezifisch sei und nicht die Wissensbasis, die dem früheren Strategiegebrauch zugrunde liegt, berühre. In den frühen Stadien der Metagedächtnis-Entwicklung scheint nur ein globales Verständnis der Nützlichkeit von Organisationsverhalten für die Erinnerungsleistung vorzuliegen, aber kein spezifisches Verständnis für die spezifische Bedeutung des Organisierens nach Kategorien. Das Sortieren nach Farben wird demnach als eine Form der möglichen Organisationsstrategien gesehen und entsprechend als prinzipiell positiv beurteilt. Wurden auch andere Aspekte des Metagedächtnisses mit einbezogen (z.B. Bewusstsein für die Bedeutung von Lernzeit, Listenlänge, Alter einer Person), so waren Hinweise darauf erkennbar, dass Kinder mit einem guten allgemeinen Metagedächtnis im Alter von 6 Jahren im Alter von 8 Jahren unter 
veränderten Aufgabenanforderungen eher eine Organisationsstrategie einsetzten, als Kinder mit weniger gutem Metagedächtnis. Unter den strategischen Vorschülern gibt es deutliche Unterschiede in Bezug auf ihr metamemoriales Verständnis für den Strategiegebrauch. Ein frühes Metagedächtnis scheint dabei zumindest tendenziell mit einer hohen Stabilität des Strategiegebrauchs zusammenzuhängen. Unterstützt wird diese Annahme dadurch, dass die Leistung im „Wellman-Interview“ nicht mit dem Strategiegebrauch im Alter von 4 und 6 Jahren $(\mathrm{r}<.10)$, sondern erst im Alter von 8 Jahren $(\mathrm{r}=.22)$ korreliert. Frühes metakognitives Wissen begünstigt demnach langfristig das strategische Verhalten, indem der Nutzen zu einem Zeitpunkt offensichtlich wird, $\mathrm{zu}$ dem die Aufgabenanforderungen wirklich strategisches Verhalten erfordern, z.B. bei zunehmender Listenlänge. Abbildung 2.6 verdeutlicht noch einmal die Komplexität des Einflusses der Metakognition auf strategisches Lernverhalten.

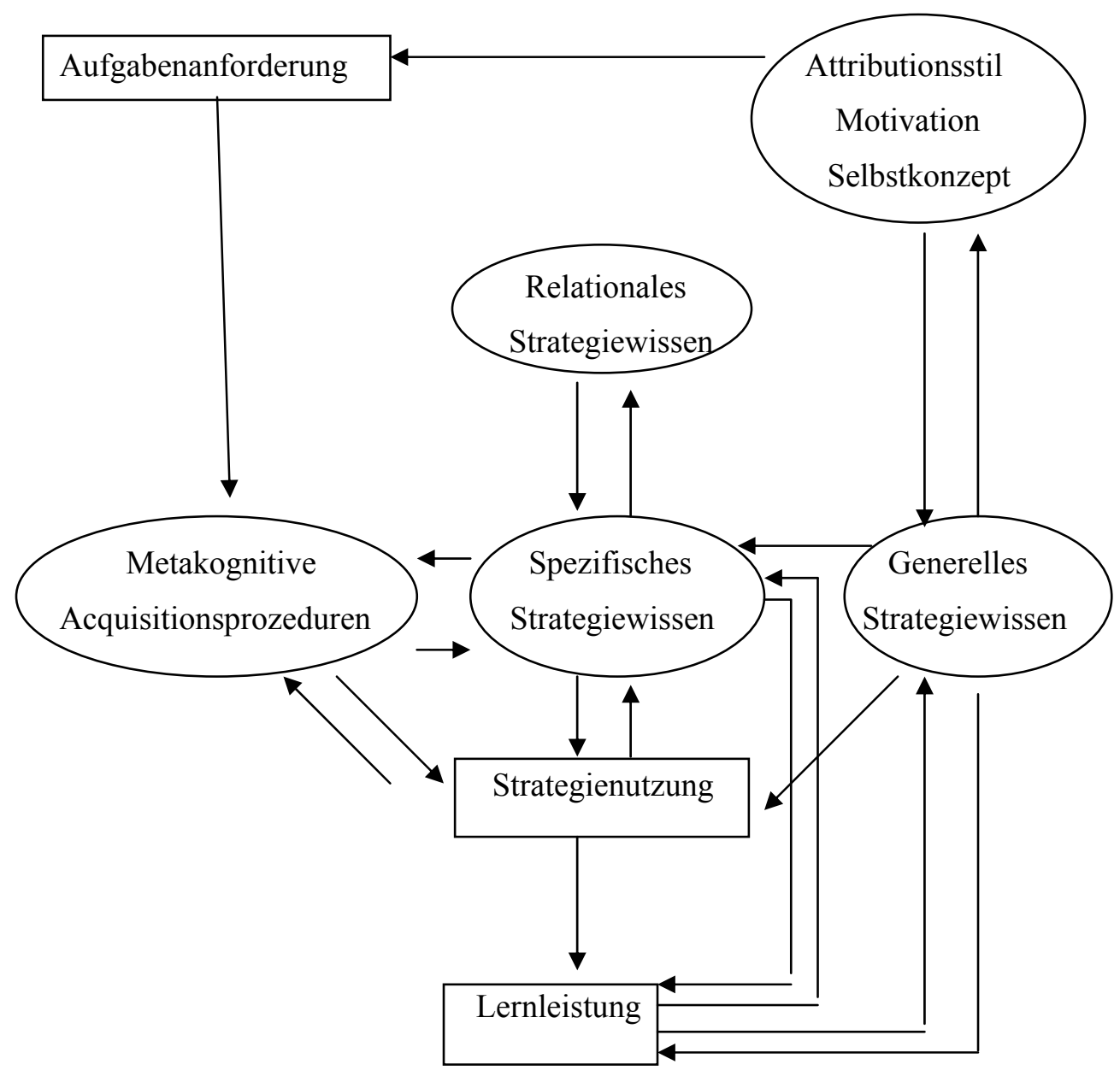

Abbildung 2.6: Modell zum Einfluss der Metakognitionen auf strategisches Lernverhalten (nach Borkowski \& Turner, 1990, S. 161) 


\section{Die Bedeutung des Metagedächtnisses für die Strategieeffektivität}

Auch bei der Beschäftigung mit der Rolle des Metagedächtnisses muss zwischen dem Einfluss des deklarativen Metagedächtnisses und prozeduralen Aspekten (Selbstüberwachungs- bzw. Selbstregulationsprozessen) differenziert werden. Ein gutes Wissen über das Gedächtnis kann das Auftreten eines Nutzungsdefizits begünstigen, indem es möglicherweise zu einem sehr frühen und möglicherweise zu frühen Gebrauch einer Strategie führt. Wenn Kinder genügend über die Anwendung einer Strategie gelernt haben und den damit regelmäßig verbundenen Nutzen einer Strategie kennen, werden sie diese Strategie auch irgendwann einmal anwenden. Die große Überzeugung von dem Nutzen der Strategieanwendung wird wahrscheinlich dazu führen, dass die Strategie auch mehrfach zum Einsatz kommt, selbst wenn es Hinweise darauf gibt, dass die Strategie ihnen nicht hilft.

Ein Kind mit schlechterem Metagedächtnis wird meistens gar nicht auf die Idee kommen, eine so komplexe Strategie anzuwenden, da es sie entweder nicht genügend kennt oder sich davon keinen Vorteil verspricht. Damit wirkt ein schlechtes Metagedächtnis dem Nutzungsdefizit entgegen. Wenn es gar nicht erst zu einem Strategieeinsatz kommt, kann sich das Verhalten auch nicht als uneffektiv erweisen (vgl. Bjorklund \& Coyle, 1995).

Das Wissen über das Gedächtnis, über Merkmale der Aufgabe, der Person, der Strategie und der Situation kann dem Auftreten eines Nutzungsdefizits aber auch entgegenwirken. Dafür muss es auf effektive Art und Weise dafür sorgen, dass die Strategie genau an die vorliegende Aufgabe angepasst wird. Dieses könnte sich beispielsweise darin äußern, dass eine selektive Gedächtnisstrategie vermehrt bei den Items eingesetzt wird, die in den vorhergehenden Durchgängen noch nicht erinnert wurden. Im Gegensatz zu jüngeren Kindern kann bei älteren Kindern diese Vorgehensweise beobachtet werden (Masur, McIntyre \& Flavell, 1973). Das bessere Verständnis der älteren Kinder über die Arbeitsweise des Gedächtnisses versetzt sie in die Lage, ihre Strategien effektiver einzusetzen.

Der Zusammenhang zwischen Metagedächtnis und der Gedächtnisleistung erwies sich in Metaanalysen insgesamt als mittelmäßig hoch (Schneider, 1985), wobei dieser Zusammenhang mit dem Alter zunahm. Metakognitive Fähigkeiten scheinen sich aber allgemein stärker auf die Anwendung von Strategien auszuwirken als auf ihre Effektivität. Bezogen auf das kategoriale Organisieren kann diese Wirkung z.B. über das Erkennen der kategorialen Listenstruktur vermittelt werden (Hasselhorn, 1986). Trotz der intensiven Beschäftigung mit dieser Thematik (z.B. Kurtz \& Weinert, 1989; Schneider, Körkel \& Weinert, 1987) scheint das Beziehungsgeflecht so komplex $\mathrm{zu}$ sein, dass sich keine 
eindeutigen, allgemeingültigen Wirkungszusammenhänge identifizieren lassen (vgl. Hasselhorn, 2001).

Die meisten Untersuchungen zum Metagedächtnis bezogen sich eher auf allgemeine Aspekte als auf aufgabenspezifische Kenntnisse. In einer Untersuchung zum spezifischen aufgabenbezogenen Metagedächtnis zeigten Fabricius und Cavalier (1989), dass das Wissen der Kinder darüber, wie Gedächtnisstrategien die Erinnerungsleistung verbessern, mit ihrem späteren Strategieeinsatz und der Auswirkung auf die Behaltensleistung zusammenhängt. Die untersuchten Kinder im Alter von 4 bis 6 Jahren sollten in der ersten Sitzung die zu lernenden Bilder benennen und nach der Wiedergabe angeben, ob und wenn ja warum das Benennen hilfreich war. Die gegebenen Begründungen ließen sich entweder einem „WahrnehmungsVerhaltens-Mechanismus (z.B. „Benennen bewirkt, dass mehr Zeit zum Betrachten der Karten da ist.“) oder einem „kognitiven Mechanismus (z.B. „Benennen hilft dabei, weiterhin über die Karten nachzudenken.“) zuordnen. Die Kinder, die ihre Reproduktionsleistung auf die Benennungsstrategie zurückführten, erinnerten mehr als die Kinder, die dies nicht taten. Die geringere Strategieeffektivität der Kinder mit schlechten metakognitiven Fähigkeiten zeigte sich eine Woche später, als die spontane Anwendung der Benennungsstrategie bei einer Gedächtnisaufgabe untersucht wurde. Der Zusammenhang zwischen dem Strategiegebrauch und der Reproduktion war bei den Sechsjährigen, die ihre Wiedergabeleistung nicht auf das Benennen zurückführten, geringer als bei den Gruppen, die dies taten und erklären konnten.

Unabhängig vom Ausmaß der Bewusstheit über die Wirkungsweise von Strategien erleichterte das Benennen bei den jüngeren Kindern nicht die Wiedergabe. Die jüngeren Kinder mit dem höchsten Grad an metakognitivem Wissen über Strategien (z.B. diejenigen, die kognitive Erklärungen abgaben) neigten dazu, das Benennen auch dann einzusetzen, wenn sich ihre Erinnerungsleistung dadurch nicht verbesserte. Die Verbesserung des Metagedächtnis scheint also zu zunehmendem Strategieeinsatz zu führen und erhöht dabei die Wahrscheinlichkeit für ein Nutzungsdefizit, da den Kindern die Anwendung der Strategie noch nicht hilft. In verschiedenen Altersstufen scheint das Metagedächtnis eine unterschiedliche Bedeutung für die Strategieeffektivität zu haben.

Wenn Kinder ihr Verhalten bei der Aufgabenbearbeitung und das erzielte Ergebnis nur unzureichend reflektieren und auch von anderen keine Rückmeldung über ihre Leistung bekommen, so kann dies zu einer Fehleinschätzung der eigenen Fähigkeiten führen. Die Annahme der jüngeren Kinder, eine gute Leistung gezeigt zu haben, kann sie darin bestärken, mit dem Gebrach der Strategie fortzufahren. Ältere Kinder, die über bessere 
Selbstregulations- und Selbstüberwachungskompetenzen verfügen, erkennen eher, wenn Schwierigkeiten bei der Wiedergabe der Items auftreten und beenden die Anwendung einer wenig hilfreichen Strategie. Nach Miller und Seier (1994) wäre demnach folgender Entwicklungsverlauf zu erwarten: Kinder mit guten Selbstüberwachungsfähigkeiten, die spontan eine wenig hilfreiche Strategie eingesetzt haben, hören mit der Strategieanwendung wieder auf und greifen zu einem späteren Zeitpunkt darauf zurück, wenn sie davon profitieren. Unklar bleibt hierbei allerdings, woher die Kinder wissen sollten, wann der richtige Zeitpunkt ist, um die Strategie erneut einzusetzen. Um zu wissen, ob die Strategie ihnen nützt, müssten sie sie erst einmal anwenden, auch auf die Gefahr hin, dass sie sich erneut als uneffektiv herausstellt. Darüber hinaus besteht die Möglichkeit, dass die ausgewählte Strategie nicht automatisch zu einem Leistungsvorteil führt, sondern dass sie geübt werden muss. Ohne das Beibehalten der Strategie werden demnach Kinder, die die Strategie infolge des ausbleibenden kurzfristigen Erfolges absetzen, auch langfristig keinen Nutzen aus der Strategie ziehen können.

Bjorklund und seine Kollegen (1994) fanden in ihrer Untersuchung, dass die meisten als nutzungsdefizitär klassifizierten Kinder über den Einsatz von Strategien Bescheid wussten. Jüngere Kinder schienen erstaunlich unempfänglich für den Erfolg oder das Versagen ihrer Strategie zu sein. Kinder im Alter von 5, 6 und 8 Jahren mit schlechten Erinnerungsleistungen änderten im folgenden Durchgang genauso häufig ihr Vorgehen wie die Kinder mit guter Reproduktionsleistung. Trotz geringer und fehlerhafter Erinnerungsleistung setzten die meisten Kinder den Einsatz einer wenig hilfreichen Strategie fort (McGilly \& Siegler, 1989).

Eine Ursache, dafür dass die angestrebte Wirkung (Effektivität) nur einen geringen Einfluss auf das Verhalten der Kinder hat, kann darin liegen, dass jüngere Kindern in Bezug auf die Güte ihrer Leistung häufig unrealistisch optimistisch sind (z.B. Bjorklund \& Green, 1992; Helmke, 1991; Stipek \& McIver, 1989). So überschätzen sie meistens die Menge der Items, die sie erinnern können (Flavell, Friedrichs \& Hoyt, 1970). Wenn ihnen Fehler rückgemeldet werden, setzen sie dieses Wissen nicht verhaltensrelevant um wie ältere Kinder (Stipek, 1984), sondern erwarten unabhängig davon weiterhin eine Verbesserung im nächsten Durchgang.

Dieser Überoptimismus hilft aber unter Umständen bei der Auflösung des oben beschriebenen Dilemmas, nicht zu wissen, wann eine Strategie nützlich wird und ob der Erfolg an eine intensive Beschäftigung mit der Strategie geknüpft ist. Aufgrund ihrer zuversichtlichen Einstellung setzen auch jüngere Kinder die Aufgabenbearbeitung fort und 
können damit ihre Fähigkeiten verbessern. Da Kinder bei der Bearbeitung einer Gedächtnisaufgabe auch bei anfänglichen Misserfolgen auf einen späteren Nutzen der Strategieanwendung vertrauen, wenden sie die Strategie weiterhin an. Entwicklungspsychologische Befunde zeigen, dass diese Hoffnung bei einigen Kindern auch begründet ist (vgl. Bjorklund et al., 1992).

Eine Überschätzung der eigenen Leistungsfähigkeit, die zur Anwendung von objektiv betrachtet ineffektiven Strategien führt, ist also langfristig für die weitere Entwicklung durchaus sinnvoll. Daher stellt sich die Frage, ob die Einschätzung der eigenen Kompetenzen aufgrund von mangelhaften kognitiven Fähigkeiten zustande kommt oder ob vielmehr motivationale Faktoren ein sehr starkes Gewicht bekommen. Selbst wenn die Kinder ihre eigene Leistung richtig einschätzen könnten, scheint es in bestimmten Entwicklungsphasen sinnvoller zu sein, sich auf künftige Erfolgserwartungen zu konzentrieren als auf aktuelle Misserfolge. Auf der Suche nach Determinanten der effektiven Strategieentwicklung müssen also neben kognitiven und metakognitiven, auch motivationale Einflussfaktoren berücksichtigt werden. Auf den kombinierter Einfluss von kognitiven und nicht-kognitiven Determinanten (Motivation und Selbstkonzept) wird im Folgenden näher eingegangen.

\subsubsection{Gemeinsamer Einfluss von kognitiven und nicht-kognitiven Determinanten}

Die Anzahl potentieller nicht-kognitiver Einflussfaktoren lässt sich kaum bestimmen. Entsprechend groß ist das Ausmaß an Kombinationsmöglichkeiten kognitiver und nichtkognitiver Faktoren und ihrer Auswirkungen auf die Entwicklung einer effektiven Strategie. Aus diesem Grund ist es erforderlich, sich auf zwei der nicht-kognitiven Determinanten zu beschränken, die in der Vergangenheit schon mehrfach in diesem Zusammenhang zu Erklärungszwecken herangezogen wurden (vgl. Pressley, Borkowski \& Schneider, 1987; 1989). Hier sollen zum einen das Selbstkonzept, zum anderen motivationale Faktoren betrachtet werden.

\section{Selbstkonzept}

Das Konzept, das eine Person von sich selber hat, spiegelt die interne kognitive Repräsentation von den eigenen Fähigkeiten und Eigenschaften wieder. Diese können global angelegt sein, aber sich auch auf ganz bestimmte Bereiche beziehen (Zanobi \& Usai, 2002). Abbildung 2.6 zeigt beispielhaft für die Konzeptionalisierung des Selbstkonzeptes ein hierarchisches Modell (vgl. Shavelson, Hubner \& Stanton, 1976). 


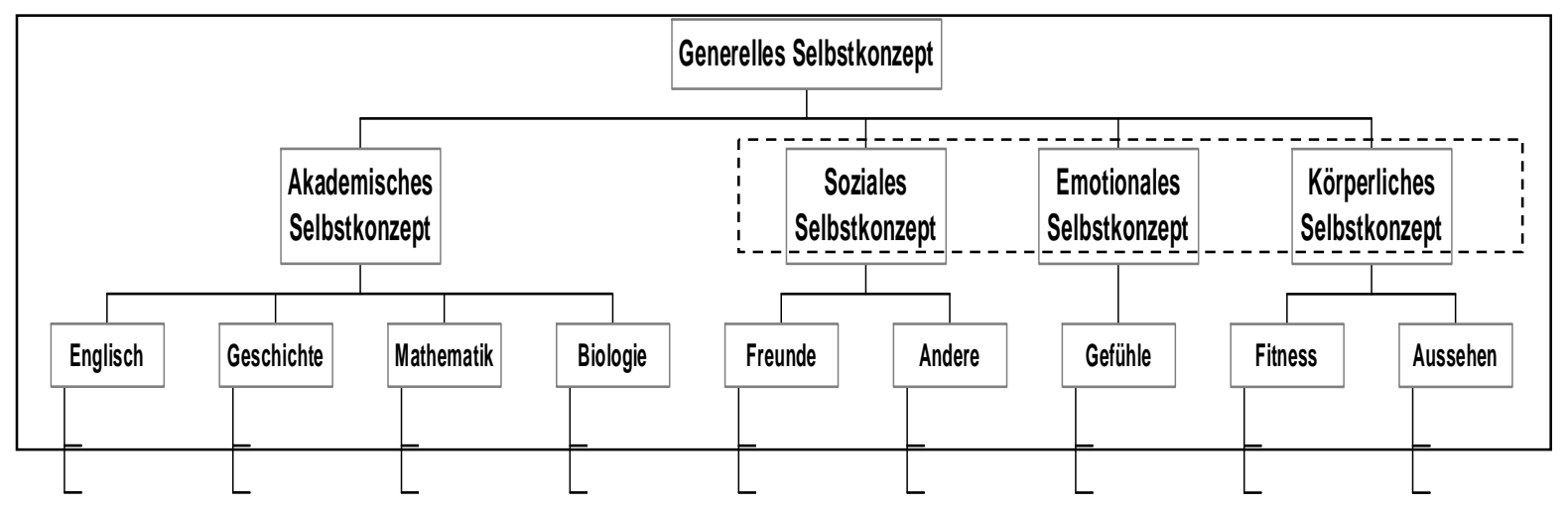

Abbildung 2.6: Das hierarchische Selbstkonzeptmodell in Anlehnung an Shavelson et al. (1976)

Darüber hinaus enthält das Selbstkonzept sowohl kognitive Aspekte (Fähigkeitsselbstkonzept) als auch affektive Elemente (Selbstwert), die als Folge der Einschätzung der persönlichen Kompetenzen entstehen können (vgl. Schöne, Dickhäuser, Spinath \& Stiensmeier-Pelster, 2003). So betonen einige Autoren den Zusammenhang mit der Entwicklung metakognitiver Kompetenzen und die Bedeutung für kognitive Leistungen (Borkowski et al., 1990), andere eher die motivationale Relevanz im Kontext der Leistungsmotivation (Helmke \& Rheinberg, 1996). Vorstellungen einer Person über sich selbst im Sinne des Selbstkonzeptes werden nach wie vor stark diskutiert sowohl was ihre Definition als auch ihre Entstehung, Struktur und Wirkung betrifft (vgl. Bong \& Clark, 1999; Krapp, 1997; Moschner, 2001). Unabhängig davon, welche Sichtweisen oder Modelle zugrunde gelegt werden (Breckler \& Greenwald, 1986; Marsh \& Yeung, 1998; Shavelson, et al., 1976), besteht ein Konsens dahingehend, dass es sich bei den unterschiedlichen Facetten der Selbsteinschätzung um parallele Entwicklungsverläufe handelt, die von einer Vielzahl von Faktoren beeinflusst werden. Neben soziokulturellen Einflüssen (Hau, Kong \& Marsh, 2000; Marsh, Kong \& Hau, 2001) zählen dazu auch geschlechtsspezifische Komponenten (vgl. Jacobs, Lanza, Osgood, Eccles \& Wigfield, 2002).

Die Grundlage für die Herausbildung des Selbstkonzeptes bilden Vergleichsprozesse mit der Leistung von anderen in dem gleichen Bereich (external frame) oder mit den eigenen Leistungen in der Vergangenheit oder in einem anderen Bereich (internal frame), wobei die Leistungsrückmeldungen in bestimmten Bereichen sich auf die Beurteilung der Fähigkeiten in anderen Bereichen auswirkt (Marsh, Byrne \& Shavelson, 1988; Möller \& Köller, 2001). Der Zusammenhang zwischen dem Selbstkonzept und der realen Leistung variiert in Abhängigkeit vom Alter und dem Fähigkeitsniveau (Krapp, 1997; Nichols, 1979). Dabei kann sowohl das Fähigkeitsselbstkonzept die Leistung beeinflussen (self-enhancement Ansatz) als auch 
umgekehrt (skill-development Ansatz), wie neuere Längsschnittstudien zeigen (Helmke \& van Aken, 1995; Stiensmeier-Pelster, Balke \& Schlangen, 1996).

\section{Relevanz für den Entwicklungsverlauf}

Eine wichtige Facette des Selbstkonzeptes betrifft die Einschätzung der eigenen Fähigkeiten im Umgang mit Lern- und Gedächtnisanforderungen. Dieses spielt bereits im vorschulischen Bereich eine Rolle (Marsh, Craven \& Debus, 1991; 1998; Marsh, Ellis \& Craven, 2002), aber natürlich auch im schulischen Kontext (vgl. zum akademischen Selbstkonzept: Marsh, 1986; 1988; 1990; Marsh et al., 1988). Ein stabiles Selbstkonzept sollte sich erst am Ende der Grundschulzeit entwickeln, wenn die Kinder als Bewertungsgrundlage nicht länger Faktoren wie Anstrengung oder soziale Verstärkung, sondern objektive und normative Informationen heranziehen (Stipek \& MacIver, 1989).

Im Laufe der Grundschulzeit kann beobachtet werden, dass die eigenen intellektuellen Fähigkeiten und die damit verbundenen Leistungserwartungen zunehmend geringer eingeschätzt werden (Helmke, 1991). Dieser vom Kindergarten bis zur 6. Klasse andauernde linear fallende Trend wird allerdings von zwei Anstiegen zu Beginn der 1. und der 3. Klasse unterbrochen. Die Einschätzung der eigenen Fähigkeiten liegt in dem gesamten Zeitraum im positiven, also im Vergleich zu Gleichaltrigen überdurchschnittlichen Bereich. Als mögliche Ursachen für diese Entwicklung werden sowohl individuelle Veränderungen (kognitive Fähigkeiten, Veränderungen im Rahmen der Schulklasse) als auch soziale Faktoren (Leistungsbewertungen, Sozialvergleiche innerhalb der Klasse) angeführt (Helmke, 1991). Insgesamt scheint es eine Entwicklung weg von einem globalen, stark situationsspezifischen und oft überoptimistischen Selbstbild hin $\mathrm{zu}$ einem differenzierteren und damit meist realistischeren Selbstkonzept zu geben (Helmke, 1998; 1999).

Damit stellt es eine wesentliche Komponente bei der Entwicklung der eigenen Lernund Leistungsmöglichkeiten dar (vgl. Pekrun, 1997; Renkl, Helmke \& Schrader, 1997; Schnabel, 1997; Skaalvik \& Valas, 1999; von Aken, Helmke \& Schneider; 1997). Nur wenn erkannt wird, wo die eigenen Grenzen liegen, ist die Grundlage dafür geschaffen, sich mit der Überwindung dieser Grenzen auseinanderzusetzen. Ansonsten scheint überhaupt keine Notwendigkeit zu bestehen, bei Gedächtnisanforderungen strategisch vorzugehen.

In der 6. Klasse scheint das akademische Selbstkonzept einen deutlichen Einfluss auf die Schulergebnisse zu haben und darüber hinaus die intrinsiche Motivation zu beeinflussen. Die intrinsische Motivation scheint hingegen für die in der Schule erzielten Leistungen von untergeordneter Bedeutung zu sein (Zanobini \& Usai, 2002). 


\section{Motivation}

An dieser Stelle wird deutlich, dass die Diskussion um den Einfluss des Selbstkonzepts auf die kognitive Entwicklung nicht geführt werden kann, ohne auf die Rolle der Motivation im Umgang mit Lern- und Gedächtnisanforderungen einzugehen (vgl. Breckler \& Greenwald, 1986; Guttentag \& Lange, 1994; Lepola \& Rauhanummi, 2000; van Kraayenoord \& Schneider, 1999). Dabei muss zwischen extrinsischer Motivierung über spezielle Anreize (z.B. Geld) und intrinsicher Motivation, d.h. die Attribuierung des Erfolgs bei einer Aufgabe auf die eigene Anstrengung bzw. die Strategieproduktion, unterschieden werden.

\section{Relevanz für den Entwicklungsverlauf}

Mit Hilfe von extrinsischer Motivierung lässt sich auch schon bei Kindern zu Beginn der Grundschulzeit das Ausmaß der Strategieproduktion beeinflussen (Gelabert, Torgesen, Dice \& Murphy, 1980; Kunzinger \& Witryol, 1984).

Die Bedeutung von intrinsischer Motivation bei der Aufgabenbearbeitung ist weniger eindeutig. Obwohl es einige Belege für einen entsprechenden Einfluss gibt (Kurtz \& Borkowski, 1987), fällt der Zusammenhang in anderen Studien geringer aus (Schneider et al., 1986) oder konnte nicht gefunden werden (Kurtz \& Weinert, 1989). Dennoch kann davon ausgegangen werden, dass durch Instruktionen oder Trainings, die für eine Attribuierung des Aufgabenerfolgs auf den Einsatz von auf die konkreten Anforderungen abgestimmten Strategien sorgen, eine dauerhafte Strategieanwendung erreicht werden kann (Borkowski \& Turner, 1990; Borkowski, Weyhing \& Carr, 1988).

\section{Zusammenhang zwischen metakognitiven und motivationalen Merkmalen}

Wenn man diese Aspekte im Hinblick auf die Entwicklung effektiver Strategien berücksichtigt, wird deutlich, dass auch entwicklungspsychologische Informationsverarbeitungsmodelle motivationale und emotionale Einflussfaktoren nicht ignorieren dürfen (vgl. Bjorklund \& Schneider, 1996; Weinert, 1984). Ab Mitte der achtziger Jahre rückte die Bedeutung und das Wechselspiel kognitiver, metakognitiver und motivationaler Merkmale bei Lern- und Problemlöseprozessen stärker in den Fokus des wissenschaftlichen Interesses (vgl. Borkowski \& Muthukrishna, 1995; Pressley, Borkowski \& O'Sullivan, 1985; Pressley, Borkowski \& Schneider, 1987, 1989; Schneider \& Pressley, 1989). In dem Modell des ,guten Informationsverarbeiters“ (Good Strategy User Model) 
wird die Güte der Lern- und Gedächtnisleistungen in direktem Zusammenhang mit der Qualität und Intensität des Strategiegebrauchs gesehen. Eine gute Informationsverarbeitung sollte demnach vor allem durch hoch entwickelte metakognitive Kompetenzen zustande kommen, was sowohl spezifisches und allgemeines Strategiewissen als auch metakognitive Überwachungs- und Kontrollprozesse und strategiebezogene Kausalattributionen beinhaltet. Bei der Anwendung von Lern- und Gedächtnisstrategien wird die Wirkung auf die Behaltensleistung erfahren, was dazu führt, dass das spezifische Wissen über den Schwierigkeitsgrad und die Situationsspezifität einzelner Strategien zunimmt und die Strategien immer besser auf die jeweiligen Anforderungen abgestimmt werden können. Dieses hat wiederum zur Folge, dass der Nutzen der Strategien immer mehr zunimmt, durch diese Erfahrung die Strategie auch beibehalten und zunehmend verfeinert wird, was zur Entwicklung eines guten generellen Strategiewissens beiträgt. Dazu zählen nicht nur kognitive Aspekte, wie die Notwendigkeit zur Erstellung von Plänen, sondern auch motivationale Faktoren, wie Anstrengungsbereitschaft, Kontrollüberzeugungen, Kausalattributionen (vgl. Borkowski, Carr, Rellinger \& Pressley, 1990; Borkowski \& Krause, 1985; Borkowski \& Mutukrishna, 1995; Borkowski \& Turner, 1990). Positive Erfahrungen beim Strategieeinsatz tragen zu einem positiven Selbstwertgefühl und damit auch zu internalen Kontrollüberzeugungen und Attributionen auf die eigene Anstrengung bei. Kognitive, metakognitive und motivationale Prozesse tragen demnach wechselseitig zur Leistungssteigerung im Umgang mit Lern- und Gedächtnisanforderungen bei (Borkowski, Johnston \& Reid, 1987; Schneider, Bjorklund et al., 1996). Abbildung 2.7 veranschaulicht diese Zusammenhänge. 


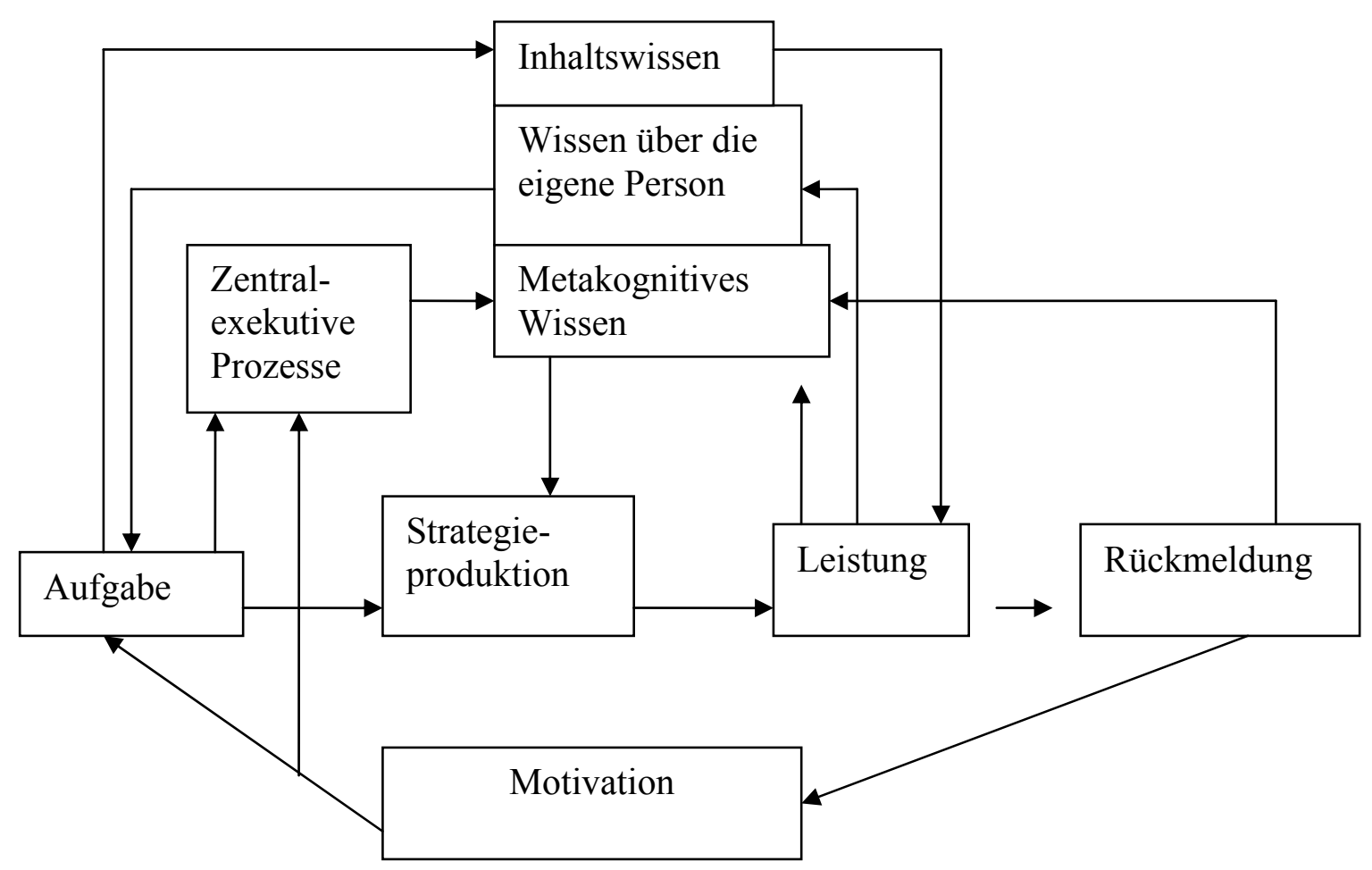

Abbildung 2.7: Metakognitives Modell der „guten Informationsverarbeitung“ (in Anlehnung an Borkowski, 1996, S. 399; Borkowski \& Thorpe, 1994, S. 54)

Empirische Belege für diese Annahme lieferten Paris, Newman und McVey (1982) in einer mikrogenetischen Studie mit sieben- und achtjährigen Kindern, die an fünf aufeinander folgenden Tagen semantische Kategorisierungsaufgaben bearbeiten sollten und am dritten Tag ein Training in der Organisationsstrategie absolvierten. Es zeigte sich, dass die Kinder, denen außerdem die Gründe für die Effektivität einer Strategie erklärt wurden, den anderen nicht nur hinsichtlich ihres Metagedächtnisses, sondern auch in Bezug auf den Strategiegebrauch und die Gedächtnisleistungen überlegen waren. Die positive Bewertung und Stabilität im Entscheidungsverhalten für eine bestimmte Strategie scheint in engem Zusammenhang mit der Einsicht und der Überzeugung der Kinder vom Nutzen der Strategie zu stehen. Andere Studien stützen diese Befunde (Fabricius \& Hagen, 1984; Pressley, Ross, Levin \& Ghatala, 1984). In Anlehnung an traditionelle „Erwartungs*Wert“-Modelle der Leistungsmotivation (vgl. Atkinson, 1982) sollte nach Paris (1988) eine bestimmte Strategie einer anderen vorgezogen werden, wenn das Produkt aus ihrem Anreizwert und ihrer Erfolgswahrscheinlichkeit größer ist als das der konkurrierenden Vorgehensweise. 
Kurtz und Borkowski (1984) untersuchten in einer Trainingsstudie die Prädiktionskraft metakognitiver und motivationaler Faktoren für den Transfer strategischen Verhaltens auf neue Aufgabenbereiche. Kinder, die ihren Erfolg auf die eigene Anstrengung zurückführten (und nicht auf unkontrollierbare Faktoren wie Aufgabenschwierigkeit), verhielten sich strategischer und hatten darüber hinaus ein besseres aufgabenbezogenes Metagedächtnis. In einem Folgeexperiment konnte gezeigt werden, dass die Attribution des eigenen Erfolgs auf die Anstrengung mit dem Strategieeinsatz bei neuen Lernanforderungen zusammenhing (Borkowski \& Krause, 1985). Aus dem Umstand, dass die Attributionsstile vor dem Strategieeinsatz erhoben wurden, wurde abgeleitet, dass der Strategiegebrauch als eine Folge der Kausalattribution anzusehen ist. Wie in der Vorläuferstudie von Kurtz und Borkowski (1984) verfügten Drittklässler, die Erfolg auf ihre Anstrengung attribuierten, über ein besseres Metagedächtnis als Kinder mit anderen Attributionsmustern. Dieses wurde von den Autoren so gedeutet, dass sie im Gegensatz zu den anderen Kindern den Zusammenhang zwischen der eigenen Anstrengung und dem Lernerfolg erkannten.

Im Rahmen einer umfangreichen Heidelberger Längsschnittuntersuchung wurde unter anderem der Zusammenhang zwischen Intelligenz, Metagedächtnis, Erfolgsmotivation, Gedächtnisstrategien und Gedächtnisleistung näher beleuchtet (Schneider, Körkel \& Weinert, 1987). Neben der Bedeutung der verbalen Intelligenz zeigte sich in den ermittelten Kausalmodellen für die Fünftklässler ein direkter Einfluss der Erfolgsmotivation auf das Metagedächtnis und die Güte der Gedächtnisleistung. Anders als in den berichteten amerikanischen Studien konnte dieser Zusammenhang für Drittklässler nicht nachgewiesen werden.

Die Abhängigkeit solcher Strukturmodelle von kulturellen und gesellschaftlichen Merkmalen zeigt sich auch in verschiedenen deutsch-amerikanischen Vergleichsuntersuchungen (Carr, Kurtz, Schneider, Turner \& Borkowski, 1989; Kurtz, Schneider, Carr, Borkowski \& Turner, 1988; Schneider, Borkowski, Kurtz \& Kerwin, 1986). Während bei den amerikanischen Viertklässlern die Anstrengungsattribution sowohl das Metagedächtnis als auch den Strategiegebrauch beeinflusste, beschränkte sich die Wirkung bei den deutschen Kindern auf das Metagedächtnis. Für deutsche Kinder hatte die intrinsische Motivation einen wesentlich höheren Stellenwert und ihre Strategieproduktion hing vor allem von ihrem Selbstkonzept ab. Letzteres hing bei amerikanischen Kindern nur leicht mit ihrem metakognitiven Wissen zusammen (Schneider, Borkowski, Kurtz \& Kerwin, 1986). Auch für andere Altersgruppen zeigte sich, dass kognitive und metakognitive Leistungen bei amerikanischen Schülern vor allem mit Anstrengungsattribution und bei 
deutschen Schülern mit Fähigkeitsattribution zusammenhängen (Carr et al., 1989; Kurtz et al., 1988). Ein Vergleich mit den Einschätzungen der Bezugspersonen der Kinder ergab, dass in den beiden Ländern sowohl die Eltern als auch die Lehrer der Kinder die gleichen Attributionsstile besaßen. Die zwischen deutschen und amerikanischen Schülern gefundenen Unterschiede beim Umgang mit Sort-Recall-Anforderungen ließen sich folglich damit erklären, dass der Nutzung kategorialer Strukturen im häuslichen und schulischen Umfeld der Kinder in den beiden Ländern ein unterschiedlicher Schwerpunkt zugesprochen wurde. Der Umgang mit Lern- und Gedächtnisanforderungen, also auch das strategische kategoriale Organisieren, scheint demnach durch erzieherische Maßnahmen beeinflussbar zu sein.

\subsubsection{Gemeinsamer Einfluss von internen und externen Determinanten}

Die Bedeutung kognitiver Einflussfaktoren kann nicht unabhängig von den realisierten Kontextbedingungen gesehen werden (vgl. Turner, Henry \& Smith, 2000). Die für eine erfolgreiche Aufgabenbearbeitung erforderlichen Voraussetzungen können beispielsweise sowohl dadurch vorliegen, dass eine Person über die notwendigen Kompetenzen verfügt als auch dadurch, dass die relevanten Informationen innerhalb der Versuchssituation zur Verfügung gestellt werden. Die Effektivität des Strategieeinsatzes hängt allerdings in den meisten Fällen von beiden Faktoren ab, d.h. ist das Ergebnis aus dem Produkt von Fähigkeiten einer Person und dem kontextbedingten Schwierigkeitsniveau der Aufgabe.

Hasselhorn (1996) betont vor allem den Zusammenhang zwischen Arbeitsgedächtniskapazität und Aufgabenmerkmalen, Wissensbasis und Lernmaterial sowie Metagedächtnis und Instruktionsmerkmalen. Die Arbeitsgedächtniskapazität fällt für Hasselhorn (1996) vor allem unter speziellen äußeren Aufgabenmerkmalen ins Gewicht. Wesentliche Kriterien sind dabei die Art der Darbietung des Lernmaterials, die zur Verfügung stehende Lernzeit, die Gestaltung des Behaltensintervalls (mit oder ohne Distraktoraufgabe) und das Ausmaß an Unterstützung durch den Versuchsleiter oder die Gestaltung der Versuchssituation.

So wird beispielsweise unter „Sort-Recall“-Bedingungen die Arbeitsgedächtniskapazität weniger beansprucht als unter „Free-Recall“-Bedingungen. Während bei der „Free-Recall“-Anforderung die zur Verfügung stehende Kapazität angesichts des knappen Zeittakts der sequentiellen Itemdarbietung vollständig zur Fokussierung der aktuell dargebotenen Information benötigt wird, besteht bei der „Sort-Recall“-Bedingung die Möglichkeit zur freien Gestaltung der Lernphase. 
Die Wissensbasis hängt für Hasselhorn (1996) in erster Linie mit der Beschaffenheit des Lernmaterials zusammen. Wenn ein sehr umfangreiches Wissen über das zu lernende Material existiert und die Lerninhalte stark miteinander assoziiert sind, so reichen oft automatische Prozesse für eine gute Erinnerungsleistung aus (vgl. Bjorklund \& Bjorklund, 1985; Chi, 1985). Selbst wenn Items entsprechend ihrer kategorialen Zuordnung erinnert werden, kann daraus nicht auf einen strategischen Abruf geschlossen werden, da alleine die Assoziationen zu dieser Wiedergabereihenfolge geführt haben können.

Allerdings kann durch die Beschaffenheit des Lernmaterials auch erreicht werden, dass das Vorliegen von Kategorien besonders deutlich wird. Wenn es sich bei den zu lernenden Items um typische Exemplare einer Kategorie handelt, so steigt die Wahrscheinlichkeit, dass die kategoriale Zugehörigkeit erkannt wird und das Item entsprechend eingeordnet wird. Damit wird durch die Materialauswahl das Erkennen einer strategischen Verhaltensmöglichkeit erleichtert, ohne dass dadurch automatisch der Einsatz einer bestimmten Lernstrategie erfolgen muss. Die Darbietung von hoch-tyischem Lernmaterial führt dementsprechend sowohl bei Schülern als auch bei Erwachsenen zu einem höheren Ausmaß an kategorialer Organisation als bei Darbietung von niedrig-typischem Lernmaterial (vgl. Rabinowitz, 1991).

Das Metagedächtnis steht für Hasselhorn (1996) in besonders engem Zusammenhang mit Merkmalen der Instruktion. Das Wissen über Lernanforderungen, das eigene Lernpotential und strategische Verhaltensmöglichkeiten soll Kinder in die Lage versetzen, sich bei einer konkreten Aufgabenstellung angemessen zu verhalten. Wenn im Rahmen der Instruktion allerdings die erforderlichen Informationen gegeben werden, ist dieses Vorwissen nicht erforderlich.

In einer „Free-Recall“-Studie von Kobasigawa (1974) führte die ausdrückliche Nennung der Kategorien, die im Lernmaterial vorkommen, und der Anzahl der Items in jeder dieser Kategorien und die Aufforderung zur Benennung der Items pro Kategorie dazu, dass sich Erstklässler in ihrer Erinnerungsleistung nicht mehr von Sechstklässlern unterschieden.

Umgekehrt lassen sich durch spezielle Instruktionen auch bestimmte Aspekte des Metagedächtnis aktivieren. Die allgemeine Erlaubnis zum Verschieben der Karten (z.B. Sodian \& Schneider, 1999) kann beispielsweise dazu anregen, über den Sinn der Manipulation an dem Lernmaterial nachzudenken und dazu führen, dass systematisch verschoben wird. In Abhängigkeit von der Qualität des Metagedächtnisses können dabei allerdings unterschiedliche Ordnungskriterien herangezogen werden. Dazu kann das 
Strukturieren nach semantischen Kategorien gehören, es kann sich aber ebenso gut um eine Anordnung nach Beliebtheit oder nach räumlichen Kriterien (z.B. in Reihen) handeln.

\subsection{Zusammenfassung}

Die bisherigen empirischen Befunde verdeutlichen die Notwendigkeit, bei der Analyse der Strategie des kategorialen Organisierens das Zusammenspiel von basalen Faktoren (Arbeitsgedächtniskapazität, Wissensbasis) und höheren kognitiven Prozessen (Metagedächtnis und Strategieeinsatz) zu berücksichtigen. Aufgrund der zunehmenden Informationsverarbeitungsgeschwindigkeit steigt die funktionale Kapazität des Arbeitsgedächtnisses. Dadurch können einzelne Items intensiver memoriert werden, was zu einer stärkeren Aktivierung der entsprechenden Knoten im semantischen Netzwerk der Wissensbasis führt und damit die Wahrscheinlichkeit dafür erhöht, dass die kategorialen Relationen eines Items so sehr aktiviert werden, dass sie den Schwellenpunkt der Aktivation überschreiten und damit in den bewusstseinszugänglichen Bereich des Arbeitsgedächtnisses gelangen. Die entdeckte kategoriale Information führt zu qualitativ anderen Kontrollprozessen durch die zentrale Exekutive. Je nachdem welche Anforderungen durch die Aufgabe, die Instruktion und das Lernmaterial an die Probanden gestellt werden, werden die begrenzten kognitiven Ressourcen mehr oder weniger in Anspruch genommen. Welcher Kapazitätsaufwand außerdem für die Durchführung der Strategie erforderlich ist, hängt davon $\mathrm{ab}$, über welche Wissensbasis und metakognitiven Kompetenzen das Kind verfügt. Wenn das Kind bereits Erfahrungen mit dieser Form von Anforderungen hat und bereits ein gewisses Automatisierungsniveau erreicht wurde, so ist die Belastung für das Arbeitsgedächtnis relativ gering. Die verbleibende funktionale Arbeitsgedächtniskapazität steht damit für die Bearbeitung der Aufgabe zur Verfügung und bestimmt die Qualität und Quantität des Ergebnisses. Die Ursachen für den veränderten Umgang mit der Bearbeitung von Aufgaben, in denen kategoriale Strukturen enthalten sind (d.h. den Einsatz kategorialer Organisationsstrategien), werden in erster Linie auf ein besseres metamemoriales Wissen zurückgeführt (vgl. Hasselhorn, 1996). Kapazitiven Aspekten und der generellen Zunahme der Informationsverarbeitungsgeschwindigkeit wird hingegen eine eher marginale Rolle zugesprochen. Ein gewisses Ausmaß kategorialen Wissens ist zwar eine Grundbedingung dafür, dass kategoriale Organisationsprozesse überhaupt stattfinden, die mit dem Alter zunehmende funktionale Kapazität des Arbeitsgedächtnisses begünstigt allerdings automatische und strategische Wissensaktivierungen in gleicher Weise. 
Entwicklungspsychologische Befunde zeigten, dass die Wissensbasis sich in erster Linie auf den Strategieeinsatz bei jüngeren Kindern auswirkt und die Leistungseinbußen infolge mangelnder Fähigkeiten abschwächen, wenn auch nicht eliminieren kann (Schneider \& Bjorklund, 1992). Offen bleibt die Frage, ob der Einfluss von Informationsverarbeitungsprozessen und Wissensaspekten in unterschiedlichen Entwicklungsabschnitten gleich bleibt oder spezifischen Veränderungen unterliegt. 


\section{Eigene Fragestellung}

\subsection{Die aktuelle empirische Datenlage}

\section{Befunde zur Entwicklung einer effektiven kategorialen Organisationsstrategie}

Obwohl grundsätzlich davon ausgegangen wird, dass der Einsatz von Lern- und Erinnerungstechniken sich positiv auf die Gedächtnisleistung auswirkt, gehen die Meinungen darüber auseinander, ab wann dieses der Fall ist und unter welchen Umständen von einem Nutzungsdefizit ausgegangen werden muss. Unterschiede in der aktuellen Befundlage sind teilweise auf die Wahl unterschiedlicher Indikatoren des Strategiegebrauchs zurückzuführen (vgl. Schlagmüller \& Schneider, 2002; Coyle \& Bjorklund, 1996), aber auch auf unterschiedliche Schwerpunktsetzungen bei der experimentellen Herangehensweise an diese Fragestellung (vgl. Miller \& Seier, 1994).

In der Vergangenheit wurden häufig verschiedene Kinder unterschiedlicher Altersgruppen miteinander hinsichtlich ihrer strategischen Kompetenzen verglichen und aus den gefundenen Altersunterschieden auf Entwicklungsveränderungen geschlossen. Da an diesen Unterschieden auch andere Einflussfaktoren beteiligt sein können, ist diese Vorgehensweise nicht geeignet, um wirkliche Entwicklungsveränderungen aufzuzeigen (vgl. Schlagmüller \& Schneider, 2002; Waters, 2000). Geeigneter für die Untersuchung intraindividueller Entwicklungsveränderungen kategorialer Organisationsprozesse sind längsschnittliche Untersuchungen. Dabei lassen sich nicht nur individuelle Entwicklungsverläufe der einzelnen Kinder beobachten sondern darüber hinaus auch strategische und nicht-strategische Kinder einer Altersgruppe $\mathrm{zu}$ verschiedenen Messzeitpunkten miteinander vergleichen, die durch die Teilnahme an der Untersuchung vergleichbare Erfahrungen mit den gestellten Anforderungen haben.

Eine umfangreiche längsschnittliche Untersuchung der Strategie des kategorialen Organisierens wurde bereits von Sodian und Schneider (1999) durchgeführt, die als LOGIKStudie bekannt ist. Obwohl die Ergebnisse dieser Studie wichtige Erkenntnisse im Zusammenhang mit der kategorialen Organisationsstrategie brachten, blieben einige Fragen unbeantwortet. In dieser Studie betrug der Abstand zwischen den Untersuchungen zwei Jahre, in denen unter Umständen entscheidende Entwicklungsprozesse stattfanden. Sodian und Schneider (1999) weisen selber darauf hin, dass weitere Längsschnittstudien erforderlich sind, die den Strategieerwerb und die Abrufleistung in kürzeren Zeitintervallen erfassen, um weitere Einblicke in den Übergangsprozess in diesem Altersspektrum zu bekommen. 
Auch Ornstein (1999) betont die Notwendigkeit der häufigeren Beobachtung und Beurteilung der Kinder, um einen wirklichen Einblick in die Entstehung und Festigung der kognitiven Fähigkeiten zu bekommen. Die mittleren Grundschuljahre sind dabei für die Konsolidierung und Ausbreitung der bewussten Erinnerungsfähigkeiten der Kinder entscheidend (z.B. Ornstein et al., 1988; Schneider \& Pressley, 1989).

Zur Untersuchung der Strategie in kürzeren Abständen führten Schlagmüller und Schneider (2002) eine mikrogenetische Studie durch. Ebenso wie Sodian und Schneider (1999) beobachteten sie einen eher abrupten Erwerb der Sortierstrategie und nur in wenigen Fällen eine kontinuierliche Verbesserung. Aufgrund der geringen Stichprobengröße ihrer Experimentalgruppe (15 Drittklässler und 7 Viertklässler) ist allerdings fraglich, inwiefern sich aus diesen Befunden allgemeine Schlussfolgerungen ableiten lassen. Darüber hinaus zeigte eine Follow-up-Untersuchung, dass Kinder, die in der mikrogenetischen Studie als Nicht-Strategen klassifiziert worden waren, ein paar Monate später die erforderliche Strategie erwarben (Schlagmüller \& Schneider, 2002). Daran wird deutlich, dass noch ein intensiver Klärungsbedarf hinsichtlich des Erwerbs strategischer Kompetenzen besteht.

\section{Befunde zu Determinanten für den effektiven Gebrauch der kategorialen Organisationsstrategie}

Insgesamt ist davon auszugehen, dass in unterschiedlichen Entwicklungsphasen verschiedene kognitive und motivationale Faktoren und Konstellationen von Determinanten die effektive Strategieanwendung und damit die Gedächtnisleistung beeinflussen. Miller und Seier (1994) hatten dazu schon Erwartungen formuliert, ohne dabei Aussagen über die differentielle Wirkung der verschiedenen Determinanten in den unterschiedlichen Phasen treffen zu können. Grundsätzlich ist davon auszugehen, dass das Metagedächtnis beispielsweise am Anfang einer Aufgabe für das Erkennen der Anforderungen und die Initiierung der Strategie verantwortlich ist, danach für die angemessene Durchführung und die Aufrechterhaltung der Strategie. In einem frühen Stadium der Strategieentwicklung sollte ein gutes Metagedächtnis das Auftreten eines Nutzungsdefizits begünstigen, da die Strategie ,zu früh" eingesetzt wird. Später ist davon auszugehen, dass ein gutes Metagedächtnis die Wahrscheinlichkeit eines Nutzungsdefizits verringert, da mit zunehmender Übung und Erfahrung das eigene strategische Verhalten besser auf die konkreten Anforderungen abgestimmt werden kann.

Hasselhorn (1996) fand in seinen Untersuchungen überzeugende Belege für den herausragenden Stellenwert des aufgabenspezifischen Metagedächtnisses für die Entwicklung 
der kategorialen Organisationsstrategie. Es zeigte sich, dass die metakognitive Kompetenz bei Sort-Recall-Aufgaben keine notwendige, aber eine hinreichende Bedingung für den Strategieeinsatz war, d.h. alle Kinder, die über ein angemessenes Metagedächtnis verfügten, waren Strategen. Bei der Free-Recall-Anforderung stellte das Metagedächtnis hingegen weder eine notwendige, noch eine hinreichende Bedingung dar. Bei diesen Ergebnissen muss allerdings beachtet werden, dass das wahre Ausmaß der metakognitiven Kompetenzen unterschätzt worden sein könnte, da es 3 - 7 Monate vor der Bearbeitung der Strategieaufgabe (Sort-Recall) erhoben wurde. In dieser Zeit könnten weitere Kinder ein angemessenes aufgabenspezifisches Metagedächtnis erworben haben, die später nicht entsprechend klassifiziert worden sind und zu einem anderen Befundmuster geführt haben (z.B. NichtStrategen mit angemessenem Metagedächtnis).

Für den Zusammenhang zwischen dem Metagedächtnis und dem Strategieeinsatz gibt es zwei unterschiedliche Vorhersagerichtungen. Die eine geht davon aus, dass das Metagedächtnis sich auf der Grundlage eines wachsenden Erfahrungsschatzes im Umgang mit Strategien ausbildet (vgl. Flavell, Miller \& Miller, 1993; Schneider \& Pressley, 1997). Im weiteren Verlauf wird darüber hinaus eine bidirektionale Beziehung unterstellt, also ein wechselseitig positiver Einfluss auch auf den Strategieerwerb (vgl. Pressley, Borkowski et al., 1989). Andere Forscher (z.B. Schlagmüller \& Schneider, 2002; Hasselhorn, 1996) gehen eher davon aus, dass das distale Metagedächtnis die Voraussetzung für das Erkennen und Einsetzen von Strategien ist (vgl. Schlagmüller \& Schneider, 2002; Hasselhorn, 1996). Sodian und Schneider (1999) führten, wie auch andere Autoren (z.B. Bjorklund \& Jacobs, 1985; Hasselhorn, 1992; Rabinowitz, 1984), die geringe Stabilität des strategischen Verhaltens, auf die möglicherweise fehlende Einsicht in die relevanten Abläufe zurück.

Das Ausmaß kognitiver Ressourcen sollte vor allem in der Anfangsphase des Strategiegebrauchs relevant sein (aktuelle Kurzzeit- und Arbeitsgedächtniskapazität), um die Aufgabenanforderungen $\mathrm{zu}$ erkennen und über eine angemessene Vorgehensweise reflektieren zu können (Miller \& Seier, 1994). Die Bedeutung der Arbeitsgedächtniskapazität sollte aber im Laufe der Zeit abnehmen, da mit zunehmender Übung und Erfahrung weniger Ressourcen in Anspruch genommen werden müssen.

Die Bedeutung der Gedächtniskapazität für die effektive Strategieentwicklung wurde von Bjorklund (1987) sehr hoch eingeschätzt. Die aktuelle Kapazität des Arbeitsgedächtnisses wurde als Indikator für die Informationsverarbeitungsgeschwindigkeit angesehen, die ausschlaggebend für die Güte der Rehearsalprozesse und damit der Einspeicherung in die 
Phonologische Schleife sein sollte. Davon abhängig wäre wiederum das Aktivationsniveau der Knoten in der Wissensbasis, das zu einem automatischen Erkennen kategorialer Relationen bzw. einer anspruchsvolleren Kontrolle der kategorialen Suchprozesse führt. Bei einer wenig elaborierten Wissensbasis kann von einer enormen Belastung der Arbeitsgedächtniskapazität durch die Strategieanwendung ausgegangen werden, die in Folge dessen zu Leistungseinbußen in der Reproduktion führt.

Hasselhorn (1996) konnte allerdings zwischen der größeren Strategieeffektivität der Zehnjährigen im Vergleich zu den Achtjährigen und der jeweiligen Arbeitsgedächtniskapazität keinen Zusammenhang feststellen. Aber auch Bjorklund und Harnishfeger (1987) konnten keine Belege dafür finden, dass die Arbeitsgedächtniskapazität für die Strategienutzung ausschlaggebend ist. In ihrem zweiten Experiment hatten Neunjährige keine größeren Leistungseinbußen bei einer Sekundäraufgabe als 13jährige. Hasselhorn (1996) interpretierte diese Befundmuster dahingehend, dass die Arbeitsgedächtniskapazität nur zur weiteren Perfektionierung der kategorialen Organisationsstrategie beiträgt, aber nicht zur Initiierung der Strategie.

Motivationale Faktoren sollten ab dem Zeitpunkt eine Rolle spielen, an dem die Kinder über die kognitiven Voraussetzungen für die Produktion der kategorialen Organisationsstrategie verfügen und die Entscheidung für den Einsatz der Strategie gefällt werden muss (vgl. Miller \& Seier, 1994). Voraussetzung dafür ist, dass die Kinder hinreichend motiviert sind (Motivation im Umgang mit Lern- und Gedächtnisanforderungen) und sich selber zutrauen, von dem gezeigten Verhalten zu profitieren (Selbstkonzept). Eine hohe Motivation, sich mit Lern- und Gedächtnisanforderungen zu beschäftigen, kann über die intensive Reflektion des eigenen Lernverhaltens zusätzlich die Ausbildung eines angemessenen Metagedächtnisses begünstigen.

Unter dem Gesichtspunkt, dass im Gesamtprozess auch die Hemmung früherer (ineffektiver) Verhaltensweisen und die Integration des Verhaltens in andere Gedächtnisaktivitäten wichtig ist, sollten auch die Faktoren aus vorausgehenden Messzeitpunkten betrachtet werden, da sie den weiteren Entwicklungsverlauf mit geprägt haben können. So kann beispielsweise der erste Eindruck, den ein Kind von dieser Aufgabenanforderung und seinen kognitiven Fähigkeiten bekommt, wichtig für die künftige Herangehensweie an vergleichbare Anforderungen sein.

Zusammenfassend kann gesagt werden, dass es bisher keine eindeutigen Belege für systematische Einflüsse einzelner Faktoren auf die effektive Strategieproduktion vor dem 10. 
Lebensjahr gibt. Hasselhorn (1996) war überzeugt, dass die wichtigsten Ursachen für den Nicht-Gebrauch der kategorialen Organisationsstrategie (Produktionsdefizit) in der Wissensbasis (im engeren Sinne das aufgabenspezifische Metagedächtnis) und einer situativen Überbelastung der Arbeitsgedächtniskapazität in Kombination mit einer mangelnden Sensitivität liegen. Daraus ergibt sich die Frage, ob bei längsschnittlichen Untersuchungen, in denen den Kindern die Anforderungen bekannt sind, bereits vor dem 10. Lebensjahr Hinweise auf die postulierten Wirkzusammenhänge für die effektive Strategieproduktion (d.h. Einfluss des Metagedächtnisses auf das effektive kategoriale Organisieren und die Rolle der Arbeitsgedächtniskapazität für die Gedächtnisleistung) finden lassen.

\section{Befunde zur Kontextabhängigkeit des Verhaltens und seiner Effektivität}

Nach Einschätzung von Hasselhorn (1996) wirkt sich der Aufgabenkontext nur in quantitativer Hinsicht, aber nicht qualitativ auf den Strategiegebrauch aus. So sollten zwar mehr Kinder die kategoriale Organisationsstrategie anwenden, die Güte der Strategieanwendung, d.h. der Bewusstseinsgrad oder die Leistungsdienlichkeit, sollte sich allerdings nicht verändern. Hinweise zur Materialabhängigkeit des kategorialen Organisierens lassen sich auch der Studie von Sodian und Schneider (1999) entnehmen, in der sowohl Modalität als auch Anzahl der Lernitems variiert wurde. Sie beobachteten dabei, dass davon das Verhalten in der Abrufphase deutlich stärker beeinflusst zu werden schien als das Verhalten in der Lernphase. Darüber hinaus fanden sie auch eine Beeinträchtigung der Reproduktionsleistung. Zur Erhöhung der Reliabilität der Untersuchung wären allerdings mehrere Durchgänge wünschenswert gewesen (vgl. Ornstein, 1999). Durch die Verwendung unterschiedlicher Lernmaterialien (Gegenstände und Bilder) kann darüber hinaus das Auftreten von strategischem Verhalten in unterschiedlichem Ausmaß gefördert werden.

Für eine gute Diagnostik der strategischen Kompetenzen eines Kindes sollte grundsätzlich versucht werden, sowohl homogene Versuchsbedingungen zu schaffen, um systematische Störeinflüsse auszuschließen, als auch die Heterogenität der Strategieanwendung in Abhängigkeit von unterschiedlichen Kontextbedingungen zu berücksichtigen. Dieser Anspruch lässt sich allerdings nur sehr schwer umsetzen. Grundsätzlich sind auch beim Einsatz von identischem Material viele Informationsverarbeitungsprozesse an der Entstehung einer Clusterleistung beteiligt, wovon einige nicht strategisch sind (Bjorklund, 1985; Lange, 1978). Das gleiche Material muss auch nicht zwangsläufig von allen Kindern auf die gleiche Art gelernt und verarbeitet werden, 
besonders wenn sie aus unterschiedlichen Altersgruppen stammen. Dennoch muss versucht werden, mit den zu unterschiedlichen Zeitpunkten erhobenen Strategiewerten annähernd das Gleiche zu messen, um Aussagen über Stabilitäten zu treffen.

\subsection{Weiterer Forschungsbedarf}

Um Antworten auf die interessierenden Fragen zu finden, scheint eine Kombination verschiedener experimenteller Herangehensweisen, die unterschiedliche Vor- und Nachteile bieten, sinnvoll zu sein (vgl. Tabelle 3).

Tabelle 3: Experimentelle Designs

\begin{tabular}{|l|l|l|}
\hline Fragestellung & Methode & Kritik \\
\hline Überprüfung von Altersdifferenzen & $\begin{array}{l}\text { Querschnitt- } \\
\text { untersuchung }\end{array}$ & $\begin{array}{l}\text { Keine Überprüfung von } \\
\text { Altersveränderungen }\end{array}$ \\
\hline $\begin{array}{l}\text { Überprüfung von } \\
\text { Altersveränderungen }\end{array}$ & $\begin{array}{l}\text { Längsschnitt- } \\
\text { untersuchung }\end{array}$ & $\begin{array}{l}\text { Vermischung erfahrener und } \\
\text { erstmaliger Strategen }\end{array}$ \\
\hline $\begin{array}{l}\text { Analyse des erstmaligen } \\
\text { (effektiven) Strategiegebrauchs }\end{array}$ & $\begin{array}{l}\text { Verlaufsanalysen } \\
\text { (Individualebene) }\end{array}$ & $\begin{array}{l}\text { keine Analyse des } \\
\text { „anfänglichen“ } \\
\text { Strategiegebrauchs }\end{array}$ \\
\hline $\begin{array}{l}\text { Analyse des ,,anfänglichen“ } \\
\text { (effektiven) Strategiegebrauchs }\end{array}$ & $\begin{array}{l}\text { Differentielle } \\
\text { Analysen (Gruppen) }\end{array}$ & $\begin{array}{l}\text { „Anfangsphase“ des } \\
\text { Strategiegebrauchs nicht } \\
\text { eindeutig bestimmbar }\end{array}$ \\
\hline $\begin{array}{l}\text { Untersuchung der Abhängigkeit des } \\
\text { (effektiven) Strategiegebrauchs } \\
\text { vom Lernmaterial }\end{array}$ & $\begin{array}{l}\text { Durchführung } \\
\text { mehrerer Durchgänge } \\
\text { + verschiedener Listen }\end{array}$ & $\begin{array}{l}\text { Konfundierung verschiedener } \\
\text { Kontexteinflüsse }\end{array}$ \\
\hline
\end{tabular}

Für die Untersuchung der Strategie des kategorialen Organisierens und seiner Leistungsdienlichkeit scheint das Sort-Recall-Paradigma sinnvoll zu sein, da bei dieser experimentellen Herangehensweise der Strategiegebrauch sowohl in der Lern- als auch in der Abrufphase beobachtet werden kann. Um Aussagen über die Stabilität von Strategiegebrauch und -effektivität treffen zu können, ist es wichtig, mehrere Durchgänge durchzuführen. Bei längsschnittlichen Untersuchungen lassen sich darüber hinaus Aussagen über Veränderungen im Entwicklungsverlauf treffen. Ein Abstand von 6 Monaten zwischen zwei Messungen ermöglicht auch das Aufdecken von vergleichsweise kurzfristigen Entwicklungsveränderungen, die in der LOGIK-Studie (Sodian \& Schneider, 1999) möglicherweise unentdeckt blieben (vgl. Ornstein, 1999). Gleichzeitig sind aber auch die 
Übungseinflüsse nicht so groß wie in mikrogenetischen Studien (z.B. Schlagmüller et al., 2001).

Um für die Kinder günstige Rahmenbedingungen für das Zeigen der Strategie zu schaffen, sollten bei der Materialauswahl bekannte Items und Kategorien ausgewählt werden und die Zeit für das Enkodieren und Abrufen der Lerninhalte großzügig bemessen sein. Die Instruktion sollte ausreichend Informationen vermitteln, um die Gestaltungsmöglichkeiten innerhalb der Lernphase zu verdeutlichen, aber nicht die Anwendung der kategorialen Organisationsstrategie provozieren. Über eine Instruktion, dass das Verschieben der Karten in der Lernphase erlaubt ist, lässt sich sicherstellen, dass alle Kinder die Möglichkeit der Manipulation des Lernmaterials kennen, ohne dass durch den Versuchsleiter oder andere Kontextbedingungen eine bestimmte Art der Aufgabenbearbeitung erzwungen wird. Auch in der Abrufphase sollte die Erinnerung der Kategorien nicht forciert, sondern die Reihenfolge der Wiedergabe des Lernmaterials freigestellt werden. Um entscheiden zu können, ob das gezeigte Verhalten von den Kindern bewusst und zielgerichtet eingesetzt wird, ist im Anschluss an die Wiedergabephase eine Nachbefragung erforderlich, bei der die Kinder das von ihnen gezeigte Verhalten begründen. Eine allgemein gehaltene Formulierung verhindert, dass besonders das kategoriale Organisieren begünstigt wird. Es ist lediglich zu vermuten, dass die Kinder durch die Frage nach dem „Warum“ ihr eigenes Verhalten stärker reflektieren.

Beim Rückschluss von einer angemessenen Begründung des Verhaltens auf einen bewussten, zielgerichteten Strategieeinsatz müssen allerdings unterschiedliche verbale Fähigkeiten und motivationale Faktoren berücksichtigt werden. Bei geringen verbalen Fähigkeiten können die Kinder ihr Wissen nicht transparent machen, was zu einer Unterschätzung der wahren Fähigkeiten führt. Die gleiche Wirkung hätte eine ausbleibende ausführliche Erklärung, weil das Kind müde oder gelangweilt ist. Zu einer Überschätzung der metamemorialen Kompetenz käme es, wenn das Kind in der Nachbefragung seinen Lernerfolg auf das Bilden der Kategorien zurückführt, ohne von dieser Kausalität überzeugt zu sein. Hintergrund wäre in diesem Fall, der Wunsch, eine positive Rückmeldung vom Versuchsleiter oder den Eltern zu bekommen. Auch unterschiedliches Vorwissen, z.B. durch Erfahrungen mit vergleichbaren Leistungsanforderungen, können einen Einfluss auf die Qualität der Bearbeitung der Sort-Recall-Anforderung gehabt haben. 


\subsection{Zielsetzung der vorliegenden Untersuchung}

In der vorliegenden Untersuchung soll die Entwicklung effektiver Strategien am Beispiel der kategorialen Organisationsstrategie untersucht werden. Unter Berücksichtigung der dargestellten Kritikpunkte, die es bei anderen Studien gab, die sich mit diesem Forschungsgebiet beschäftigten, sollen folgende Kriterien beachtet werden:

- Angemessenheit der experimentellen Anforderung (d.h. Untersuchung der kategorialen Organisationsstrategie mit dem Sort-Recall-Paradigma)

- Längsschnittliche Untersuchung des Erwerbs und der Entwicklung der kategorialen Organisationsstrategie bei einer größeren Stichprobe

- Untersuchung in kleinen Untersuchungsabständen (d.h. mehrmals im Jahr)

Die definierten Ziele liegen im Wesentlichen in der Klärung von zwei übergeordneten Fragestellungen, die sich folgendermaßen formulieren lassen:

\section{Fragestellungen zum Phänomen des Nutzungsdefizits}

- Welche intraindividuellen Entwicklungen sind beim kategorialen Organisieren beobachtbar? Wie typisch ist dabei das Auftreten der Phase des Nutzungsdefizits?

- Welche interindividuellen Unterschiede in den intraindividuellen Veränderungen der Anwendung der kategorialen Organisationsstrategie treten auf? Welche Aussagen lassen sich dabei hinsichtlich der Effektivität des Verhaltens treffen?

\section{Fragestellungen zu Determinanten eines effektiven Strategiegebrauchs}

- Welche Bedingungen und Auslöser der individuellen Entwicklungsprozesse sind identifizierbar?

- Warum kommt es zu interindividuellen Unterschieden in den intraindividuellen Veränderungen der Anwendung der kategorialen Organisationsstrategie?

\section{Ad 1: Zum Phänomens des Nutzungsdefizits}

Hier geht es darum, die Befunde zum Auftreten bzw. Ausbleiben des Nutzungsdefizits mit Hilfe einer Längsschnittstudie mit kürzeren Untersuchungsabständen zu überprüfen. Unter der Voraussetzung, dass das Phänomen des Nutzungsdefizits zu beobachten ist, sollen Aussagen darüber getroffen werden, in welchen Phasen dieses der Fall ist. 
Grundsätzlich stellt sich dabei die Frage, ob das Phänomen des Nutzungsdefizits ein Hinweis darauf ist, dass strategisches Verhalten prinzipiell zunächst einmal nicht die Gedächtnisleistung verbessert oder ob es eher eine (seltene) Phase im Entwicklungsprozess ist, die nur bei wenigen Kindern und nur unter bestimmten Umständen zu beobachten ist. Zur Beantwortung dieser Fragen soll zwischen verschiedenen Facetten des Nutzungsdefizits differenziert werden. Neben der Leistungsdienlichkeit von allgemeinem kategorialen Organisationsverhalten wird die Effektivität, mit der eine bewusste Kategorisierungsstrategie produziert wird, untersucht. Dabei werden verschiedene Beweise des Nutzungsdefizits getrennt voneinander betrachtet, wie Altersunterschiede bzw. Altersveränderungen, eine ausbleibende Leistungssteigerung infolge des ersten Strategieeinsatzes und einem geringeren Nutzen des Strategiegebrauchs in der Anfangsphase (im Vergleich zum erprobten Strategiegebrauch).

Das Phänomen des Nutzungsdefizits kann sich auf unterschiedliche Art und Weise äußern:

- $\quad$ Ältere Kinder zeigen eine größere Strategieeffektivität als jüngere Kinder.

Dieses spiegelt sich in Altersunterschieden, aber auch in Altersveränderungen wieder.

Da altersbedingte Unterschiede im strategischen Verhalten allerdings mit einer Vielzahl anderer alterskorrelierter Unterschiede im kognitiven und motivationalen Bereich konfundiert sind, sind Unterschiede im individuellen Entwicklungsverlauf aussagekräftiger:

- Ineffektivität des 1. Strategieeinsatzes: Eine spontan produzierte Strategie hilft anfänglichen Strategen nicht und kann sie sogar behindern.

- Ineffektivität der anfänglichen Strategieproduktion: Die Behinderung der Wiedergabe durch den spontanen Strategiegebrauch betrifft nur die Anfangsphase. Mit zunehmender Erfahrung, d.h. in einem späteren Durchgang, steigt der Nutzen, der von dem Strategieeinsatz ausgeht.

\section{Ad 2: Determinanten für das effektive kategoriale Organisieren im Grundschulalter}

Die Ursachen für eine geringe Effektivität des Einsatzes von Strategien können bei unterschiedlichen Strategien und in unterschiedlichen Altersstufen sehr verschieden sein 
(Miller \& Seier, 1994). Während man sich in einer frühen Entwicklungsphase fragen kann, warum eine wenig nützliche Strategie überhaupt eingesetzt wird, geht es in späteren Entwicklungsabschnitten eher um die Frage, warum jüngere und ältere Kinder von der gleichen Strategie in unterschiedlichem Ausmaß profitieren. Bestimmte Einflussfaktoren sollten in den verschiedenen Stadien der Strategieentwicklung (Bewertung, Anbahnung, Durchführung und Aufrechterhaltung der Strategie, Informationsverarbeitung) eine spezifische Wirkung und einen unterschiedlichen Stellenwert haben.

Einen entscheidenden Motor für die Entwicklung effektiver Strategien sehen Hasselhorn (1996) und Schneider (1986) in einem gut ausgebildeten Metagedächtnis. Das aufgabenspezifische Metagedächtnis sollte in allen Entwicklungsphasen eine Rolle spielen, d.h. sowohl bei der Strategiebewertung, -einleitung, -durchführung und -aufrechterhaltung als auch bei der Integration der Strategie in andere Gedächtnisaktivitäten.

Darüber hinaus betonen verschiedene Forscher die Bedeutung des Wechselspiels von kognitiven, metakognitiven und motivationalen Faktoren für die Entstehung strategischer Kompetenzen (z.B. Borkowski, Johnston \& Reid, 1987; Schneider, 1986). Die Gedächtniskapazität ist besonders in der Anfangsphase des Strategiegebrauchs, also von der Entscheidung bis zum Einsatz der Strategie, und für die weitere Verarbeitung der Informationen von großer Bedeutung. Ein Fehlen an mentalen Ressourcen kann nach Miller und Seier (1994) zu der Produktion wenig effektiver Strategien (Nutzungsdefizit) führen. In kulturübergreifenden Vergleichsuntersuchungen wurde darüber hinaus deutlich, dass für deutsche Kinder die intrinsische Motivation und das Selbstkonzept bei der Entwicklung strategischer Kompetenzen einen hohen Stellenwert einnehmen (Schneider et al., 1986).

Allerdings sollte nicht nur der aktuelle kognitive und emotionale Zustand einer Person relevant sein, sondern auch auch die frühere Verfassung und die bisher gesammelten Erfahrungen bei der Bearbeitung der Aufgabenanforderungen. Erfolgserlebnisse im Zusammenhang mit der Strategieproduktion sollten sich positiv auf das Selbstwertgefühl auswirken und über entsprechende Attributionsmuster zu einer Leistungssteigerung beitragen. Frühere Befunde zeigten bereits den Zusammenhang zwischen der Einsicht von Kindern in die Nützlichkeit einer Strategie und dem Entscheidungsverhalten bei der Aufgabenbearbeitung (Fabricius \& Hagen, 1984; Paris et al., 1982; Pressley et al., 1984).

Hasselhorn (1996) führt die entwicklungsbedingte Zunahme der Gedächtnisleistung zu einem Großteil auf die verbesserten strategischen Kompetenzen zurück. Darüber hinaus stellt sich allerdings die Frage, ob neben der Strategieproduktion auch andere Faktoren eine Rolle für die Güte der Gedächtnisleistung spielen. 
Mit Hilfe der vorliegenden Untersuchung soll überprüft werden, ob sich die in der Strategie-Emergenz-Theorie (Hasselhorn, 1996) postulierten Zusammenhänge bereits bei jüngeren Kindern finden lassen, wenn diese mit den Anforderungen vertraut sind. Dem aufgabenspezifischen Metagedächtnis wird dabei eine besondere Bedeutung für die Strategieentwicklung eingeräumt, während die Arbeitsgedächtniskapazität sich positiv auf die allgemeine Gedächtnisleistung auswirken sollte. Die Analyse von relevanten kognitiven Bedingungen (z.B. Gedächtniskapazität und Metagedächtnis) soll dabei u.a. klären, ob das Auftreten oder Ausbleiben von Nutzungsdefiziten auf spezifische Entwicklungskonstellationen der Arbeitsgedächtniskapazität und/oder des Metagedächtnisses zurückführbar ist. Darüber hinaus soll auf die Bedeutung motivationaler Prozesse und leistungsbezogener Facetten des Selbstkonzepts für die Entwicklung strategischer Kompetenzen eingegangen werden. Konkret geht es darum, zu explorieren, unter welchen Bedingungen in welchen Altersabschnitten und bei welchen kognitiven Voraussetzungen individuelle Besonderheiten des Fähigkeits-Selbstkonzeptes die Genese strategischer Kompetenzen fördern oder behindern. Auf die Bedeutung des kombinierten Einflusses von motivationalen und kognitiven Prozessen für die Strategieentwicklung wurde in der letzten Zeit vermehrt hingewiesen (z.B. Schneider, 1996; Schneider \& Bjorklund, 1996).

Ergänzend zu den kognitiven (Kurzzeit- und Arbeitsgedächtniskapazität) und metakognitiven Leistungsmerkmalen sollen auch allgemeine intellektuelle Fähigkeiten, die sich in Form von verbalen Kompetenzen (z.B. Wortschatz) niederschlagen, nicht vernachlässigt werden.

\section{Exkurs: Determiniertheit effektiven kategorialen Organisierens}

Die Analyse der relevanten Bedingungen, unter denen kategoriales Organisieren gezeigt wird und sich als leistungsdienlich erweist, schließt ein, dass auch die realisierten Kontextbedingungen näher beleuchtet werden. Von besonderer Bedeutung könnte dabei beispielsweise die Vertrautheit mit den Anforderungen und Merkmale des verwendeten Lernmaterials sein. Hasselhorn (1996) zeigte in seinen Untersuchungen, dass ungünstige Aufgabenmerkmale einen Einfluss auf die Aufgabenbearbeitung haben können. Dieser äußerte sich darin, dass in der schwierigeren „Free-Recall“-Aufgabe weniger Kinder die Organisationsstrategie einsetzten als in der „Sort-Recall“-Aufgabe. Wenn das Verhalten allerdings gezeigt wurde, so waren keine qualitativen Einbußen beobachtbar, d.h. es wurde angemessen begründet und erwies sich als leistungsdienlich. Darüber hinaus schien auch die 
Beschaffenheit des Lernmaterials eine Rolle zu spielen. Dieses wird daran deutlich, dass nur bei einer hohen kategorialen Typizität der Items in der „Sort-Recall“"-Aufgabe die Mehrheit der Viertklässler strategisch organisierte (vgl. Studie 8). Ein restriktiver Einfluss auf das Organisationsverhalten ging hingegen von Lernmaterial aus, bei dem nicht-kategoriale Assoziationen zwischen den Items stark ausgeprägt waren (vgl. Studie 9). In dem Zusammenhang wurde auch die Bedeutung interner Faktoren vor dem Hintergrund kontextueller Restriktionen untersucht. Dabei zeigte sich, dass sich unter kapazitätsentlastenden Bedingungen und einer indirekten Aufforderung zum Strategiegebrauch fast alle Viertklässler mit angemessenem aufgabenspezifischem Metagedächtnis strategisch verhielten. Die Befunde von Hasselhorn (1996) machen deutlich, dass strategisches Verhalten nur unter Berücksichtigung der kontextuellen Rahmenbedingungen interpretiert werden kann, wobei dem Schwierigkeitsgrad der Aufgabe ein besonderer Stellenwert zukommt. Dabei scheinen sowohl die Versuchssituation als auch die Beschaffenheit des Lernmaterials (z.B. Eindeutigkeit der kategorialen Zuordnung) eine Rolle zu spielen.

Sodian und Schneider (1999) konnten darüber hinaus zeigen, dass auch die Modalität und die Anzahl des Lernmaterials einen Einfluss auf das kategoriale Organisieren bei einer „Sort-Recall“-Aufgabe haben kann. Eine Umstellung des Lernmaterials von Spielzeug auf Bildkarten, die gleichzeitig mit einer Erhöhung der Itemanzahl verbunden war, wirkte sich bei Achtjährigen in Form eines Rückgangs des kategorialen Organisierens in der Abrufphase und der Reproduktionsleistung aus. Allerdings blieb unklar, welche Auswirkungen diese Veränderungen zu einem späteren Zeitpunkt gehabt hätten.

In der vorliegenden Untersuchung soll der Frage nachgegangen werden, ob die Beschaffenheit des Lernmaterials einen Einfluss auf das kategoriale Organisieren in einem zusätzlichen Durchgang hat, d.h. wenn die Anforderungen bereits vertraut sind. Aufgrund der bisherigen Befunde soll dabei sowohl auf die Auswahl (vgl. Hasselhorn, 1996) als auch auf die Anzahl der Items (vgl. Sodian \& Schneider, 1999) geachtet werden, wobei beide Faktoren in verschiedenen Entwicklungsstadien eine unterschiedliche Bedeutung haben sollten. Die Auswahl der Items könnte in einer frühen Entwicklungsphase noch eine Rolle spielen. Später sollte dieses Merkmal nur noch eine marginale Rolle spielen, besonders wenn die Aufgabenanforderungen bekannt sind. In dieser Phase könnten eher andere Faktoren (z.B. Lernmenge) einen Einfluss haben. Eine umfassende Berücksichtigung aller potentiellen Kontexteinflüsse beim kategorialen Organisieren und seiner Leistungsdienlichkeit ist allerdings kaum möglich und wird auch nicht angestrebt. Die vorgenommenen Betrachtungen 
haben eher explorativen Charakter und sollen zum Ausdruck bringen, dass die Befunde zur Entwicklung des kategorialen Organisierens und seiner Effektivität stets vor dem Hintergrund dieser kontextuellen Besonderheiten zu sehen sind.

Aus pragmatischen Gründen scheint es daher angemessen, sich in unterschiedlichen Altersstufen auf Aspekte des Lernmaterials zu konzentrieren, von denen eine Beeinflussung der Produktion und Effektivität des kategorialen Organisierens erwartet werden kann. Dazu gehören der Einfluss der Itemauswahl in der Mitte der Grundschulzeit und der Einfluss der Itemanzahl am Ende der Grundschulzeit.

\section{Konkretisierung der Fragestellungen}

Die konkreten Fragestellungen lassen sich demnach folgendermaßen formulieren:

\section{Ad 1. Fragestellungen zum Phänomen des Nutzungsdefizits}

- Ist die intraindividuelle Entwicklung der kategorialen Organisationsstrategie eher durch einen kontinuierlichen oder durch einen sprunghaften Verlauf beschreibbar?

- Stellt das Nutzungsdefizit im intraindividuellen Entwicklungsverlauf ein typisches Phänomen dar?

- Unterscheiden sich Kinder hinsichtlich des Zeitpunkts, zu dem sie die kategoriale Organisationsstrategie entdecken oder der Konsistenz, mit der sie kategorial organisieren?

- Welche Aussagen kann man angesichts der interindividuellen Unterschiede in der intraindividuellen Entwicklung über die Effektivität des strategischen Verhaltens treffen?

\section{Ad 2. Fragestellungen zu Determinanten eines effektiven Strategiegebrauchs}

- Welche Bedeutung hat das aufgabenspezifische Metagedächtnis alleine oder zusammen mit anderen kognitiven oder motivationalen Faktoren für die Entwicklung des kategorialen Organisierens?

- $\quad$ Besteht ein Zusammenhang zwischen dem Auftreten eines Nutzungsdefizits und der Entwicklungskonstellation des Metagedächtnisses und/oder der Arbeitsgedächtniskapazität?

- Welche internen Voraussetzungen sind in welchen Altersabschnitten für das Anwenden der Kategorisierungsstrategie und seine Effektivität erforderlich?

- Unter welchen Bedingungen kommt es bei welchen Voraussetzungen in bestimmten Altersstufen zum effektiven Einsatz der kategorialen Organisationsstrategie? 


\section{Methode}

Die vorliegende Studie wurde im Rahmen eines DFG-Projektes zur Erforschung der Bedingungen und intraindividuellen Entwicklungsverläufe strategischer Gedächtnisprozesse zwischen 5 und 12 Jahren durchgeführt. Realisiert wurde ein kombiniertes QuerschnittLängsschnitt-Design, auf das im folgenden Abschnitt noch näher eingegangen wird.

\subsection{Stichprobe und Rahmenplan}

Die Stichprobenrekrutierung erfolgte an Grundschulen und Orientierungsstufen des Göttinger Stadtgebietes und Landkreises sowie in Einbeck. Voraussetzung für die Teilnahme der Kinder an der Studie war das schriftliche Einverständnis der Eltern und die Bereitschaft, die Kinder zur Untersuchung zu bringen.

Die Studie wurde im Frühjahr 2001 mit insgesamt 157 Kindern begonnen. Davon waren 111 Kinder aus der eigentlichen Längsschnittstichprobe (Zweitklässler) und 46 Kinder aus der Querschnittstichprobe (je 23 Viert- und Sechstklässler). In die endgültige Datenanalyse gingen von der Längsschnittstichprobe zu den ersten vier Messzeitpunkten die Werte von 86 Kindern, zum fünften Messzeitpunkt von 82 Kindern ein. Zum fünften Messzeitpunkt setzte sich die Querschnittstichprobe aus 33 Zweit-, 21 Viert- und 37 Sechstklässlern zusammen. Die Verteilung des durchschnittlichen Alters und des Geschlechts über die verschiedenen Klassenstufen wird aus Tabelle 4.1 ersichtlich. 
Tabelle 4.1: Mittleres Alter der Untersuchungsteilnehmer (in Jahren, Standardabweichung in Klammern), Anzahl $(\mathrm{N})$ und Geschlechterverteilung ( $\mathrm{m}=$ männlich; $\mathrm{w}=$ weiblich) für die verschiedenen Klassenstufen

\begin{tabular}{|c|c|c|c|c|c|}
\hline Klasse & \multicolumn{2}{|c|}{ Alter (Jahre; Monate) } & $\mathrm{N}$ & \multicolumn{2}{|c|}{ Geschlecht } \\
\hline \multirow{5}{*}{$\begin{array}{l}\text { Längsschnitt- } \\
\text { stichprobe }\end{array}$} & Ende Klasse 2 & $\mathrm{M}=8 ; 4(0 ; 66)$ & \multirow[t]{4}{*}{86} & \multirow[t]{4}{*}{$\mathrm{m}=34$} & \multirow[t]{4}{*}{$\mathrm{w}=52$} \\
\hline & Anfang Klasse 3 & $\mathrm{M}=8 ; 9(0 ; 64)$ & & & \\
\hline & Ende Klasse 3 & $\mathrm{M}=9 ; 2(0 ; 64)$ & & & \\
\hline & Anfang Klasse 4 & $\mathrm{M}=9 ; 8(0 ; 63)$ & & & \\
\hline & Ende Klasse 4 & $\mathrm{M}=10 ; 2(0 ; 57)$ & 82 & $\mathrm{~m}=32$ & $\mathrm{~W}=50$ \\
\hline \multirow{2}{*}{$\begin{array}{l}\text { Querschnitt- } \\
\text { stichprobe } \\
\text { (zum 1. MZP) }\end{array}$} & Ende Klasse 4 & $\mathrm{M}=10 ; 4(0 ; 6)$ & 23 & $\mathrm{~m}=13$ & $\mathrm{w}=10$ \\
\hline & Ende Klasse 6 & $\mathrm{M}=12 ; 2(0 ; 5)$ & 23 & $\mathrm{~m}=7$ & $\mathrm{w}=16$ \\
\hline \multirow{3}{*}{$\begin{array}{l}\text { Querschnitt- } \\
\text { stichprobe } \\
\text { (zum 5. MZP) }\end{array}$} & Ende Klasse 2 & $\mathrm{M}=8 ; 9(0 ; 57)$ & 33 & $\mathrm{~m}=17$ & $\mathrm{w}=16$ \\
\hline & Ende Klasse 4 & $\mathrm{M}=10 ; 3(0 ; 65)$ & 21 & $\mathrm{~m}=9$ & $\mathrm{w}=12$ \\
\hline & Ende Klasse 6 & $\mathrm{M}=12 ; 1(0 ; 5)$ & 37 & $\mathrm{~m}=23$ & $\mathrm{w}=14$ \\
\hline
\end{tabular}

Im halbjährlichen Abstand wurden jeweils ca. zweistündige Individualuntersuchungen durchgeführt. Wie in Abschnitt 4.2 noch näher ausgeführt wird hatten die teilnehmenden Kinder ein weites Spektrum an Gedächtnisanforderungen $\mathrm{zu}$ bearbeiten. Neben der Arbeitsgedächtniskapazität (phonetischer Speicher, zentral-exekutive Koordinationsfähigkeit) wurden auch metakognitive Aspekte (Strategiewissen, Monitoring) und motivationale Faktoren (Motivation und Selbstkonzept eigener Lern- und Gedächtnisfähigkeiten) erhoben. Der unten abgebildete Versuchsplan gibt einen Überblick über die durchgeführten Messzeitpunkte mit den jeweiligen Stichproben:

Messzeitpunkt:

T1 Frühjahr 2001

T2 Herbst 2001

T3 Frühjahr 2002

T4 Herbst 2002

T5
Frühjahr 2003

\section{Längsschnittstichprobe}

Ende 2. Klasse

Anfang 3. Klasse

Ende 3. Klasse

Anfang 4. Klasse

Ende 4. Klasse

\section{Querschnittstichprobe}

4. Klasse

2. Klasse

4. Klasse 


\subsection{Versuchsanforderungen und -durchführung}

Die Untersuchungen fanden in Versuchsräumen der Abteilung für Pädagogische Psychologie und Entwicklungspsychologie des Georg-Elias-Müller-Instituts für Psychologie der Universität Göttingen statt. In zweistündigen Individualuntersuchungen, unterbrochen von einer ca. 15minütigen Pause, wurden den Kindern verschiedene Gedächtnisanforderungen gestellt, die sie in einer festgelegten Reihenfolge zu bearbeiten hatten. Die Kombination erfolgte dabei so, dass sowohl Synergie- als auch Ermüdungseffekte weitgehend vermieden wurden. Diese Aufgaben wurden im halbjährlichen Abstand wiederholt (vgl. Tabelle 4.2).

Tabelle 4.2: Aufgabenübersicht zu den jeweiligen Messzeitpunkten:

\begin{tabular}{|c|c|c|c|c|c|c|}
\hline & \multicolumn{2}{|c|}{2001} & \multicolumn{2}{|c|}{2002} & 2003 \\
\hline & & Frühjahr & Herbst & Frühjahr & Herbst & Frühjahr \\
\hline \multirow{3}{*}{$\begin{array}{l}\text { Sort- } \\
\text { Recall- } \\
\text { Aufgabe }\end{array}$} & Liste 1 & \multicolumn{5}{|c|}{20 Items (je 4 aus 5 Kategorien) } \\
\hline & \multirow[t]{2}{*}{ Liste 2} & \multicolumn{2}{|c|}{20 Items (je 4 aus 5 Kategorien) } & \multicolumn{2}{|c|}{$\begin{array}{l}25 \text { Items (je } 5 \text { aus } 5 \\
\text { Kategorien) }\end{array}$} & $\begin{array}{l}30 \text { Items (je } 5 \text { aus } \\
6 \text { Kategorien) }\end{array}$ \\
\hline & & \multicolumn{5}{|c|}{ altersspezifisch konstante Typizitätswerte } \\
\hline \multicolumn{2}{|c|}{$\begin{array}{l}\text { Metagedächtnis- } \\
\text { Fragebogen }\end{array}$} & $\begin{array}{l}\text { Items aus dem } \\
\text { Fragebogen } \\
\text { von } \\
\text { Schlagmüller } \\
\text { et al. (2001) }\end{array}$ & $\begin{array}{l}\text { Items aus dem } \\
\text { Erhebungs- } \\
\text { instrument von } \\
\text { Belmont und } \\
\text { Borkowski } \\
(1988)\end{array}$ & \multicolumn{3}{|c|}{$\begin{array}{l}\text { Items aus dem Fragebogen von } \\
\text { Schlagmüller et al. }(2001)+\text { Listenitem } \\
\text { von Belmont und Borkowski (1988) }\end{array}$} \\
\hline \multicolumn{2}{|c|}{$\begin{array}{l}\text { Zahlen- } \\
\text { nachsprechen }\end{array}$} & \multicolumn{5}{|c|}{ Zahlennachsprechen vorwärts + Zahlennachsprechen rückwärts } \\
\hline \multicolumn{2}{|c|}{$\begin{array}{l}\text { Selbstkonzept- } \\
\text { Fragebogen }\end{array}$} & \multicolumn{5}{|c|}{ Köpfchenliste (altersspezifische Itemmodifikation) } \\
\hline \multicolumn{2}{|c|}{$\begin{array}{l}\text { Motivations- } \\
\text { fragebogen }\end{array}$} & \multicolumn{5}{|c|}{ Smiley-Liste (altersspezifische Itemmodifikation) } \\
\hline \multicolumn{2}{|c|}{$\begin{array}{l}\text { Verbale } \\
\text { Fähigkeiten }\end{array}$} & & $\begin{array}{l}\text { Wortschatz- } \\
\text { Test }\end{array}$ & \multicolumn{3}{|c|}{\begin{tabular}{|l|} 
Test zum \\
Allgemeinen \\
Wissen
\end{tabular}} \\
\hline
\end{tabular}




\subsubsection{Semantische Organisationsaufgabe („Sort-Recall“-Aufgabe)}

Das Lernmaterial (vgl. Anhang A) bestand bei den ersten beiden Messzeitpunkten aus zwei unterschiedlichen Sets mit je 20 Bildkarten $(4,5$ x 4,5 cm), auf denen Objekte ausgewählter Kategorien mit den jeweiligen Objektbezeichnungen abgebildet waren. Der erste Satz enthielt Items aus den Kategorien Büromaterialien, Gepäckstücke, Haushaltsgeräte, Medizinartikel und Lebensmittel. Die Items des zweiten Satzes konnten den Kategorien Obst, Kleidungsstücke, Möbel, Werkzeuge und Körperteile zugeordnet werden. Bei der Itemauswahl wurde unter Heranziehung der Normen von Hasselhorn, Jaspers und Hernando (1994) auf vergleichbare Typizitätswerte sowohl zwischen den einzelnen Kategorien als auch zwischen den unterschiedlichen Altersstufen geachtet (vgl. Anhang B). Während die Items aus dem ersten Set über alle Messzeitpunkte konstant eingesetzt wurden, wurden im zweiten Set die Items so ausgewählt, dass für jede Altersstufe vergleichbare mittlere Typizitätswerte resultierten. Dadurch waren die in verschiedenen Altersstufen eingesetzten Listen nicht identisch. Ab dem dritten Messzeitpunkt wurde die Itemanzahl bei der zweiten Liste auf 25 erhöht, um Deckeneffekte zu vermeiden.

\section{Exkurs: Materialunterschiede}

Die Besonderheit der zweiten Liste bestand darin, dass diese zum 1. und 2. Messzeitpunkt deutlich höhere Typizitätswerte aufwies. Ab dem 3. Messzeitpunkt wurde die Typizität des Lernmaterials an die Typizität der ersten Liste angepasst, dafür wurde aber die Gesamtzahl der zu lernenden Items heraufgesetzt.

Die kategoriale Typizität der Items der ersten Liste weicht $\mathrm{zu}$ den ersten beiden Messzeitpunkten deutlich voneinander $\mathrm{ab}(\mathrm{t}(2)=26,25, \mathrm{p}=.001)$. Die kategorialen Typizitätswerte für die einzelnen Items in den verschiedenen Altersstufen sind dem Anhang zu entnehmen.

\section{Exkurs: Auswahl der Kategorien und Kategoriebeispiele}

Den Kindern war Lernmaterial vorgelegt worden, dass nach Kategorien geordnet werden konnte. Da den Kindern nicht mitgeteilt wurde, um welche Kategorien es sich handeln sollte, musste überprüft werden, ob diese Einteilung auch von den Kindern gewählt wird oder ob Kinder ein anderes Gliederungskriterium bevorzugen. Die höchste Abweichung im Sortierverhalten hinsichtlich der vorgegebenen Kategorien gab es am Ende der dritten Klasse, wo 7 der 86 untersuchten Kinder (d.h. 8,14\%) 
Büroartikel und ein „Behältnis“ (meistens „Rucksack“) zu einer Gruppe zusammenfügten. $\mathrm{Zu}$ allen anderen Messzeitpunkten lag der Anteil der Kinder, die das Lernmaterial in anderen Gruppen zusammenfügte, als die die vorgegeben waren, unter $5 \%$ (d.h. maximal 4 Kinder).

\section{Instruktion}

Vor der Präsentation des Lernmaterials informierte der Versuchsleiter das Kind über die bevorstehende Aufgabe, indem er allgemeine Aufgabenmerkmale („Spiel mit Bildern“) und den Ablauf (inkl. der zur Verfügung stehenden Lernzeit) schilderte und explizit auf das Ziel der Aufgabe, d.h. das Merken von möglichst vielen Bildern, hinwies.

Die Kärtchen eines Sets wurden vor dem Kind in einer festgelegten Anordnung (,Kärtchenwolke“) hingelegt, wobei sichergestellt war, dass keine Kärtchen aus derselben Kategorie nebeneinander lagen (vgl. Abbildung 4.1). Dabei wurden die Objekte gemäß der Objektbezeichnungen auf den Karten von dem Versuchsleiter benannt.

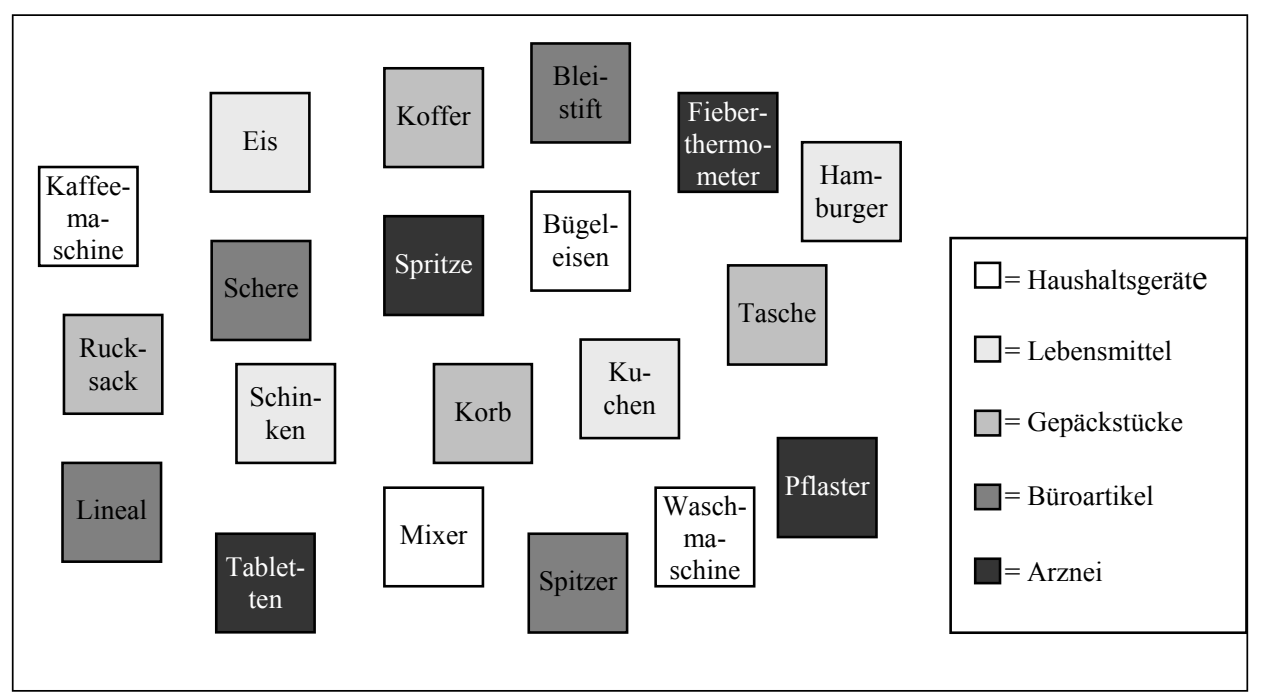

Abbildung 4.1: Präsentation des Materials zu Beginn der Lernphase einer Sort-RecallAufgabe (,Kärtchenwolke“).

Im Anschluss daran erhielten die Kinder eine unspezifische Instruktion, indem sie darauf hingewiesen wurden, dass sie beim Lernen alles tun dürften, was ihrer Meinung nach für das Merken der Karten hilfreich wäre (vgl. Abbildung 4.2). 


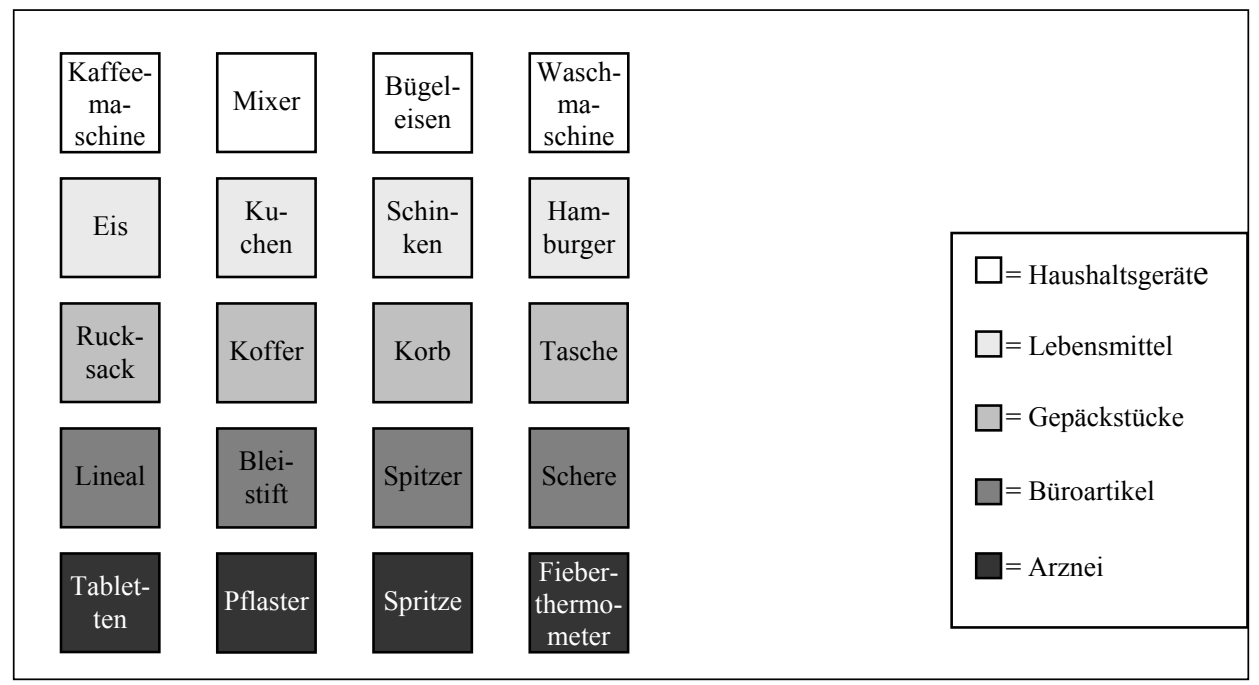

Abbildung 4.2: Beispiel für strukturierte Anordnung des Materials am Ende der Lernphase einer Sort-Recall-Aufgabe.

Falls das Kind nach der Hälfte der zur Verfügung stehenden Lernzeit (3 Minuten) immer noch mit einer Manipulation am Lernmaterial beschäftigt war, erhielt es vom Versuchsleiter den erneuten Hinweis auf die Notwendigkeit des zusätzlichen Lernens der Items. Nach Ablauf der Lernzeit wurden die Karten abgedeckt und eine 30-sekündige Distraktoraufgabe durchgeführt (Rückwärts zählen), um potentielle Recency-Effekte zu unterbinden. Daran schloss sich die Abrufphase an, in der der Versuchsleiter die Reproduktionsleistung des Kindes protokollierte. Dabei stand dem Kind erst einmal beliebig viel Zeit zur Verfügung. Wenn ein Kind allerdings 10 Sekunden lang kein weiteres Item nannte, so wurde es vom Versuchsleiter noch einmal zum Nachdenken angeregt, bis dieser nach weiteren 10 Sekunden ohne Itemnennung den Versuch beendete.

\section{Exkurs: Ausschluss möglicher Effekte durch Hilfestellungen des Versuchsleiters}

Die instruktionsgemäße Aussage „Stell dir noch mal vor, wie die Kärtchen alle da lagen“ legt allerdings unter Umständen den Einsatz einer räumlichen Strategie nahe. Die Überprüfung des von den Kindern gezeigten Verhaltens nach dieser Instruktion zeigte allerdings, dass von dieser Aussage keine systematische Beeinflussung ausgeht. Nur bei wenigen Kindern führte die Aufforderung, sich erneut mit dem Lernmaterial auseinanderzusetzen, kaum zu einer weiteren Steigerung der Erinnerungsleistung. Der Anteil der Kinder, die nach der Instruktion mehr als ein neues Item erinnern konnten, lag zwischen $8-16 \%(8,1-12,8 \%$ in Liste $1 ; 8,1-16,3 \%$ in Liste 2$)$. Die räumliche Nähe der Karten nutzen dabei weniger als $6 \%$ der Kinder. 
Zur Erfassung des metamemorialen Bewusstseins fand zum Abschluss nach jedem Durchgang eine Nachbefragung bezüglich des Lernverhaltens des Kindes statt („Kannst du mir sagen, wie du dir das alles merken konntest?"). Dabei wurden vom Versuchsleiter alle Begründungen des Kindes für die jeweiligen Verhaltensweisen wörtlich protokolliert.

\section{Auswertung des kategorialen Organisationsgrades}

Zur Quantifizierung von Organisationstendenzen wurde der von Roenker, Thompson und Brown (1971) entwickelte „Adjusted Ratio of Clustering“ (ARC) verwendet.

Dieses Maß schnitt in der Simulationsstudie von Murphy (1979), in der die Konfundierung der Organisationsmaße mit der Reproduktionsleistung überprüft wurde, noch besser ab als der „Ratio of Repetition“ (RR), der von der Arbeitsgruppe von Bousfield (Bousfield \& Bousfield, 1966) entwickelt wurde. Während sich das Grundmodell des RR als Zufallsanordnung der dargebotenen Items mit anschließender Zufallsanordnung beschreiben lässt, handelt es sich beim ARC um eine Zufallsanordnung der tatsächlich reproduzierten Items (vgl. Pellegrino, 1975).

$A R C=(R-$ Erw $) /($ Max - Erw $)$

In diesem Quotienten steht im Zähler die Differenz zwischen der Anzahl intrakategorialer Wiederholungen $(\mathrm{R})$ und dem aufgrund der reproduzierten Items ermittelten Erwartungswert von R (Erw), im Nenner die Differenz zwischen dem maximal erreichbaren R (Max) und dem Erwartungswert von R (Erw). Als intrakategoriale Wiederholungen wird dabei die aufeinanderfolgende Wiedergabe von Items aus der gleichen Gruppe (Kategorie) bezeichnet. Der Wertebereich des ARC liegt zwischen -1 und 1, mit 0 als Zufallswert und 1 als Maximalwert.

Die Nachteile des ARC, dass er bei Reproduktion nur einer Kategorie nicht definiert ist und negative Werte schwer interpretierbar sind, treten gegenüber seinen Vorteilen in den Hintergrund (Murphy \& Puff, 1982). Dazu gehört u.a., dass der Zufalls- und der Maximalwert unabhängig von der Zusammensetzung der Lernlisten sind, so dass Vergleiche zwischen unterschiedlichen Listen möglich sind. Für die hier dargestellte entwicklungspsychologische Studie mit ihren Untersuchungsanforderungen ist daher der ARC geeigneter. Die Gefahr, dass nur Items aus einer Kategorie erinnert werden und damit der ARC nicht definiert ist, ist darüber hinaus in der untersuchten Altersgruppe äußerst gering. 


\subsubsection{Fragebogen zur Erfassung des aufgabenspezifischen Metagedächtnisses}

Die Erfassung des aufgabenspezifischen Metagedächtnisses orientierte sich an dem Vorgehen von Schneider, Schlagmüller und Visé (1998) und dem von Hasselhorn (1994). Am Ende der zweiten und ab dem Ende der dritten Klasse wurden Items aus der Würzburger Testbatterie zum deklarativen Metagedächtnis (Schlagmüller et al., 2001; Schneider, Schlagmüller \& Visé, 1998) ausgewählt, die das Wissen über semantische Kategorisierungsstrategien erfassten. Die vorgegebenen Antwortalternativen mussten von den Kindern durch die Vergabe von Noten oder Rangplätzen bewertet werden. Darüber hinaus wurden sie aufgefordert, eine differenzierte Begründung für die getroffene Entscheidung abzugegeben (vgl. Anhang C und D).

Bei der Auswertung wurde die Korrektheit der von den Kindern aufgestellten Rangordnung zwischen den Antwortalternativen über Paarvergleiche bestimmt. Ein Punkt wurde vergeben, wenn die Entscheidung eindeutig zugunsten des Organisierens nach semantischen Kriterien ausfiel. Das gleiche Kriterium wurde für die Punktevergabe bei den freien Begründungen angelegt. Ein Kind erhielt einen Punkt, wenn in der Erklärung der Hinweis auf Kategorisierbarkeit des Lernmaterials auftauchte, und zwei Punkte, wenn darüber hinaus die inhaltlichen Kriterien erwähnt wurden. Der maximal erreichbare Wert lag bei 6 Punkten.

Ab Beginn der dritten Klasse wurde das Verständnis der Lernerleichterung durch kategoriale Ordnungsstrukturen entsprechend der Vorgehensweise von Hasselhorn (1994) überprüft. Die Grundlage bildete ein von Belmont und Borkowski (1988) entwickeltes Messinstrument, das entsprechend der realisierten Anforderungen modifiziert worden war. Um den Einfluss von Lesekompetenzen zu minimieren wurde das Wortmaterial durch dazugehörige Bilder ergänzt. Die Aufgabe für die Kinder bestand darin, eine kategorisierte, längere Liste und eine nicht kategorisierte, kürzere Wortliste sowie eine nicht kategorisierte, längere Liste hinsichtlich ihres Schwierigkeitsgrades beim Lernen miteinander zu vergleichen. Die Anzahl der Items pro Liste wurde dabei so gewählt, dass ein dem Alter angemessenes Schwierigkeitsniveau vorlag ( 8 bzw. 9 Begriffe). Darüber hinaus wurde bei der Auswahl der Begriffe und Kategorien darauf geachtet, dass das Schwierigkeitsniveau in der untersuchten Altersgruppe konstant blieb und Überschneidungen mit dem Lernmaterial aus anderen Aufgaben der Gesamtstudie vermieden wurden (je drei Fahrzeuge, Obstsorten und Tiere). Die drei Listen sollten hinsichtlich der Lernschwierigkeit in eine Rangreihe gebracht und entsprechend begründet werden. 
Als korrekte Entscheidung wurde gewertet, wenn die kategorial angeordnete Liste als leichter zu lernen eingeschätzt wurde als die unstrukturierten Listen. In die Punktevergabe floss nur der Vergleich mit der kategorisierbaren Liste ein, nicht der Vergleich der unstrukturierten Listen miteinander. Für jede richtige Entscheidung erhielt das Kind einen Punkt, d.h. zwei Punkte pro Item. Für eine Begründung, in der die Lernerleichterung auf die Kategorisierbarkeit der entsprechenden Liste zurückgeführt wurde, wurde bei jedem Item zusätzlich ein Punkt vergeben. Demnach konnte ein Kind maximal sechs Punkte erreichen.

\section{Exkurs: Güte der Messinstrumente in der vorliegenden Untersuchung:}

Da die Skala zum kategorialen Organisieren aus dem Schlagmüller-Fragebogen (Schlagmüller et al., 2001) in der vorliegenden Untersuchung insgesamt schlechte Reliabilitätswerte (Cronbachs $\alpha$ ) aufwies (Ende K1. 2: $\alpha=.22$; Ende K1. 3: $\alpha=.39$; Anfang K1. 4: $\alpha=.45$; Ende K1. 4: $\alpha=.66$ ), wurden für weitere Aussagen über das deklarative Metagedächtnis die Listenitems aus dem Messinstrument von Hasselhorn (1994) herangezogen (Anfang Kl. 3: $\alpha=.48$; Ende Kl. 3: $\alpha=.38$; Anfang Kl. 4: $\alpha=.53$; Ende Kl. 4: $\alpha=$.76). Die am Ende der zweiten Klasse eingesetzten Wortlisten und die Listenitems korrelierten stets bedeutsam miteinander $(r>.29, p<\alpha)$. Darüber hinaus erbrachten die Wortlisten befriedigende Reliabilitätswerte (Cronbachs $\alpha=.75$ bzw. $\alpha=.58)$.

Die Reliabilitätswerte (Cronbachs $\alpha$ ) des MG-Messinstruments ließen sich durch Kombination der Wortlisten des Schlagmüller-Fragebogens und der Listen von Hasselhorn deutlich steigern (Ende K1. 3: $\alpha=.59$; Anfang Kl. 4: $\alpha=.69$; Ende K1. 4: $\alpha=$.76). Aus diesem Grund wurde zur Bestimmung der metakognitiven Kompetenzen dieses Maß herangezogen.

Die Interraterreliabilität für die freien Antworten (Begründungen) erwies sich als ausreichend. Für die Wortlisten-Items lag Cronbachs $\alpha$ insgesamt bei $\alpha=.80$ (Ende Kl. 2: $\alpha=.94$; Ende K1. 3: $\alpha=.88$; Anfang K1. 4: $\alpha=.84$; Ende K1. 4: $\alpha=.64$ ), für die Litenitems bei $\alpha=.90$ (Anfang K1. 3: $\alpha=.81$; Ende K1. 3: $\alpha=.73$; Anfang K1. 4: $\alpha=.77$; Ende K1. 4: $\alpha=.65)$.

\subsubsection{Gedächtnisspannen-Aufgabe}

Zur Bestimmung der Gedächtniskapazität wurde eine Zahlenspannen-Aufgabe durchgeführt (vgl. Anhang E). Als Indikator für die Kurzzeitgedächtniskapazität wurde die 
Zahlenspanne vorwärts, als Indikator für die Arbeitsgedächtniskapazität die Zahlenspanne rückwärts genommen. In Anlehnung an den Hamburg-Wechsler-Intelligenztest für Kinder (HAWIK-III, Tewes, Schallberger \& Rossmann, 2000) wurden den Kindern über einen CDPlayer Ziffernfolgen mit einer Frequenz von einer Ziffer pro Sekunde präsentiert, um eine einheitliche Darbietung zu gewährleisten. Anders als beim HAWIK wurden nur einsilbige Ziffern verwendet, d.h. die „Sieben“ wurde durch „Null“ ersetzt. Die Aufgabe der Kinder bestand darin, diese Ziffern in derselben Reihenfolge (Zahlennachsprechen vorwärts) oder der umgekehrten Reihenfolge (Zahlennachsprechen rückwärts) wiederzugeben. Das Zahlennachsprechen rückwärts wurde immer im Anschluss an das Zahlennachsprechen vorwärts durchgeführt. Die Aufgabe wurde jeweils abgebrochen, wenn das Kind in einer Aufgabe zweimal nacheinander die Zahlenreihe nicht oder falsch replizierte.

\section{Exkurs:}

Sowohl die Kurzzeitgedächtniskapazität (Zahlennachsprechen vorwärts) als auch die Arbeitsgedächtniskapazität (Zahlennachsprechen rückwärts) erwiesen sich als interindividuell sehr stabil, d.h. korrelieren über die verschiedenen Messzeitpunkte bedeutsam miteinander ( $\mathrm{rs} \geq .38$ bzw. $\mathrm{rs} \geq .35$, ps $<.005$ ). Der Zusammenhang beider Maße zu demselben Messzeitpunkt fiel allerdings erst ab dem Ende der dritten Klasse bedeutsam aus ( $\mathrm{rs} \geq .40, \mathrm{ps}<\alpha)$, aber noch nicht am Ende der zweiten $(\mathrm{r}=.22, \mathrm{p}=.04)$ und zu Beginn der dritten Klasse $(r=.28, \mathrm{p}=.01)$.

\subsubsection{Aufgaben zur Erfassung des Selbstkonzeptes und der Motivation}

\section{Erfassung des Selbstkonzeptes der eigenen Lern- und Gedächtnisfähigkeiten}

In Anlehnung an die LOGIK-Studie, in der das Selbstkonzept der Kinder von Helmke (1999) untersucht wurde, wurde die sogenannte „Köpfchenliste“ eingesetzt. Die Aufgabe der Kinder bestand darin, die eigenen Fähigkeiten im Vergleich zu einer Bezugsgruppe (hier: Schulklasse) einzuschätzen. Auf einer 25stufigen Skala sollten die Kinder ihre relative Position hinsichtlich spezieller Kompetenzen aus dem schulischen Bereich (Aufsatz, Diktat, Lesen, Mathematik, Sachkunde, Lieder-Lernen), bei Gedächtnisanforderungen im Freizeitbereich (z.B. Memory-Spielen) und in untersuchungsnahen Gedächtnisanforderungen (Bilder-Merken, Zahlen-Merken, Wörter-Merken, Geheimwörter-Merken, Nacherzählen von Geschichten) einschätzen (vgl. Anhang G). 


\section{Erfassung der Motivation für Lern- und Gedächtnisanforderungen}

In Analogie zur Erfassung des Selbstkonzeptes der eigenen Lern- und Gedächtnisfähigkeiten wurden die Kinder gefragt, wie viel Spaß ihnen Lern- und Gedächtnisanforderungen in speziellen Bereichen machen. Dazu gehörten neben Schulfächern (Deutsch, Mathematik, Sachkunde, Musikunterricht), Lernspiele (Memory, Einkaufsspiele, Kofferpacken) und Aufgaben aus der laufenden Untersuchung (z.B. Merken von Bildern, Zahlen, Wörtern, Geschichten, etc.). Auf einer fünfstufigen Skala sollten sie ihre Bewertung von „,sehr viel Spaß“ bis ,überhaupt keinen Spaß“ abgeben (vgl. Anhang F).

\section{Exkurs: Güte der Messinstrumente in der vorliegenden Untersuchung}

Der Selbstkonzeptfragebogen erbrachte am Ende der zweiten Klasse $(\alpha=.62)$ und zu Beginn der dritten Klasse ( $\alpha=.68)$ zufriedenstellende Reliabilitätswerte (Cronbachs a), ab dem Ende der dritten Klasse fielen die Werte erheblich besser aus (Ende Kl. 3: $\alpha=.84$; Anfang K1. 4: $\alpha=.83$; Ende K1. 4: $\alpha=.78$ ).

Der Motivationsfragebogen erbrachte am Ende der zweiten Klasse mit $\alpha=.60$ akzeptable Reliabilitätswerte (Cronbachs $\alpha$ ), ab der dritten Klasse fielen die Werte besser aus (Anfang K1. 3: $\alpha=.71$; Ende K1. 3: $\alpha=.81$; Anfang K1. 4: $\alpha=.84$; Ende K1. 4: $\alpha=.81)$.

\subsubsection{Aufgaben zur Erfassung der verbalen Fähigkeiten}

Verbale Kompetenzen wurden über zwei Untertests des Verbalteils des HamburgWechsler-Intelligenztests für Kinder (HAWIK-III, Tewes, Schallberger \& Rossmann, 2000) erfasst: „Wortschatztest“ und „Allgemeines Wissen“. Für beide Untertests enthält die Testanweisung detaillierte Start- und Abbruchkriterien. Durch unterschiedliche Einstiegsstufen für die verschiedenen Altersgruppen und die Umkehrregel, nach der unter bestimmten Voraussetzungen auch Items nach abnehmender Schwierigkeit vorgegeben werden, wird eine relativ ökonomische Differenzierung erreicht (Renner \& Fricke, 2001). Für die Auswertung enthält der Verbalteil umfangreiche Lösungstabellen. Danach ist nicht nur die Bestimmung von IQ-Werten und Prozenträngen für den Gesamttest möglich, sondern auch die Vergabe von Wertpunkten $(\mathrm{M}=100 ; \mathrm{SD}=15)$ getrennt für jedes Untertestalter.

Der Wortschatz-Test (WS) verlangt vom Kind die eigenständige Definition von vorgegebenen Begriffen (z.B. „Was ist ein Hut?“, „Was bedeutet Teilnehmen?“). Eine 
Antwort, aus der auf eine gute Kenntnis des Begriffes geschlossen werden kann, wird mit 2 Punkten bewertet. Wenn das Kind eine eingeschränkte Kenntnis des Begriffes hat, so erhält es noch einen Punkt. Die Kriterien für die Punktevergabe sind in der Lösungsbewertung des Testheftes festgeschrieben und werden mit Antwortbeispielen illustriert. Insgesamt sind maximal 60 Punkte erreichbar. Der Untertest wird abgebrochen, wenn das Kind bei vier aufeinanderfolgenden Aufgaben keine oder eine falsche Antwort gegeben hat (vgl. Anhang $\mathrm{H})$.

Beim Test zum „Allgemeinen Wissen“ (AW) besteht die Anforderung in der mündlichen Beantwortung von Wissensfragen aus verschiedenen Bereichen (z.B. „Welcher Monat kommt nach März?“, „Wie wird der Sauerstoff in der Luft erneuert?“). Für jede richtige Antwort gibt es einen Punkt, wobei eine Antwort auch als richtig gewertet wird, wenn ein Kind im Nachhinein noch eine richtige Antwort zu einer früher gestellten Frage gibt. Die Bewertungskriterien sind ebenfalls dem Testheft $\mathrm{zu}$ entnehmen. Die maximal erreichbare Punktzahl beträgt 30 Punkte. Das Abbruchkriterium ist erreicht, wenn bei fünf aufeinanderfolgenden Aufgaben keine richtige Antwort gegeben wird (vgl. Anhang I). 


\section{Ergebnisse}

Im Folgenden soll versucht werden, Anworten auf die zuvor formulierten Fragen zum Phänomen des Nutzungsdefizits und zu den Determinanten eines effektiven Strategiegebrauchs zu finden.

$\mathrm{Zu}$ diesem Zweck werden zuerst die Effektivität des kategorialen Organisationsverhaltens und anschließend die Effektivität der bewussten kategorialen Organisationsstrategie überprüft, bevor weiter differenziert wird und die Leistungsdienlichkeit des ersten Strategiegebrauchs und der anfänglichen Strategieproduktion untersucht wird. Später wird den Gründen für die unterschiedliche Effektivität des Strategiegebrauchs in verschiedenen Entwicklungsphasen nachgegangen. Als dominierende Einflussgröße wird zuerst die Bedeutung des aufgabenspezifischen Metagedächtnisses untersucht. Danach wird die Wirkung des Metagedächtnisses in Kombination mit anderen kognitiven und motivationalen Faktoren untersucht. Ergänzend wird auf die Bedingungen eingegangen, unter denen das kategoriale Organisieren in unterschiedlichen Altersstufen gezeigt wurde. Dieses geschieht in der Form, dass auf Besonderheiten im intraindividuellen Entwicklungsverlauf oder den interindividuellen Unterschieden beim kategorialen Organisieren vor dem Hintergrund der Bedeutung der Vertrautheit mit den Anforderungen unter Berücksichtigung der kategorialen Typizität der Items und der Anzahl des Lernmaterials eingegangen wird.

Differenziertere Analysen wurden ab einer ausreichenden Gruppengröße durchgeführt (5\% der Gesamtstichprobe). Die Auswertung der Daten erfolgte über Korrelationsanalysen und Varianzanalysen mit anschließenden Mittelwertsvergleichen (t-Tests). Wenn nicht anders berichtet, wurde für die durchgeführten Tests das Signifikanzniveau auf $\alpha=.05$ gesetzt. Da der t-Test nach Bortz (1993) relativ robust auf potentielle Voraussetzungsverletzungen durch kleine Stichprobengrößen reagiert, wurde er auch zur Überprüfung von Unterschiedshypothesen eingesetzt, wenn der Stichprobenumfang klein war. 


\subsection{Beobachtungen zum Phänomen des Nutzungsdefizits}

Die Fragestellungen zum Phänomen des Nutzungsdefizits lauten:

- Ist die intraindividuelle Entwicklung der kategorialen Organisationsstrategie eher durch einen kontinuierlichen oder durch einen sprunghaften Verlauf beschreibbar?

- Stellt das Nutzungsdefizit im intraindividuellen Entwicklungsverlauf ein typisches Phänomen dar?

Zur Beantwortung dieser Fragen wird auf die verschiedenen Facetten, in denen sich ein Nutzungsdefizit zeigen kann, eingegangen. Diese lassen sich folgendermaßen definieren:

- $\quad$ Ältere Kinder profitieren weniger vom kategorialen Organisieren als jüngere Kinder. Dieses kann sich in Form von Altersunterschieden und/oder (intraindividuellen) Altersveränderungen äußern.

- $\quad$ Eine spontan produzierte Strategie hilft anfänglichen Strategen nicht und kann sie sogar behindern (Ineffektivität des 1. Strategieeinsatzes)

- Die Behinderung der Wiedergabe durch den spontanen Strategiegebrauch betrifft nur die Anfangsphase. Mit zunehmender Erfahrung, d.h. in einem späteren Durchgang, steigt der Nutzen, der von dem Strategieeinsatz ausgeht (Ineffektivität der anfänglichen Strategieproduktion)

Darüber hinaus sollen Antworten auf diese Fragestellungen gefunden werden:

- Unterscheiden sich Kinder hinsichtlich des Zeitpunkts, zu dem sie die kategoriale Organisationsstrategie entdecken, oder der Konsistenz, mit der sie kategorial organisieren?

- Welche Aussagen kann man angesichts der interindividuellen Unterschiede in der intraindividuellen Entwicklung über die Effektivität des strategischen Verhaltens treffen?

In diesem Zusammenhang soll der Frage nachgegangen werden, wie wichtig der frühe

Gebrauch der kategorialen Organisationsstrategie ist und welche Bedeutung die kontinuierliche Anwendung der Strategie für den weiteren Entwicklungsverlauf hat.

Ergänzend wird auf Besonderheiten im intraindividuellen Entwicklungsverlauf oder interindividuellen Unterschieden und der Effektivität des strategischen Verhaltens eingegangen, die im Zusammenhang mit Aspekten der Vertrautheit mit den Anforderungen oder der Beschaffenheit des Materials (kategoriale Typizität, Itemanzahl) stehen. 


\subsubsection{Ineffektives kategoriales Organisieren}

\section{Altersdifferenzen (Querschnittliche Analyse)}

Beantwortet werden sollte die Frage, ob kategoriales Organisieren bei älteren Kindern effektiver abläuft als bei jüngeren.

Um zu überprüfen, ob die kategorialen Strukuren in den verschiedenen Phasen der Aufgabenbearbeitung genutzt werden, wurde das Organisationsverhalten in der Lernphase getrennt von dem in der Abrufphase betrachtet. Das kategoriale Organisieren in der Lernphase wird im Folgenden auch als „Sortieren“ bezeichnet, das kategoriale Organisieren beim Abruf der Items als „Clustern“. Als kategoriale Organisierer gelten die Kinder, deren ARC-Werte im positiven Bereich liegen.

In allen Altersstufen konnte kategoriales Organisieren beobachtet werden (vgl. Tabelle 5.1). In den höheren Altersstufen war der Anteil allerdings höher als bei den jüngeren Kindern. In der Lernphase zeigten allerdings nur die Sechstklässler mehrheitlich Sortierverhalten. In der Abrufphase war in allen Altersstufen bei der Mehrheit der Kinder kategoriales Organisieren erkennbar.

Tabelle 5.1: Häufigkeitsverteilung der kategorial organisierenden Kinder in Abhängigkeit von der Phase der Aufgabenbearbeitung und dem Messzeitpunkt

\begin{tabular}{|c|c|c|c|}
\hline Kategoriale Organisierer & Zweitklässler & Viertklässler & Sechstklässler \\
\hline in der Lernphase & $\mathrm{N}=22(25,6 \%)$ & $\mathrm{N}=18(40,9 \%)$ & $\mathrm{N}=31(53,4 \%)$ \\
\hline in der Abrufphase & $\mathrm{N}=55(64 \%)$ & $\mathrm{N}=36(81,8 \%)$ & $\mathrm{N}=49(81,7 \%)$ \\
\hline
\end{tabular}

Aus dieser Beobachtung ergab sich die Frage, ob sich die kategorialen Organisierer in den verschiedenen Altersstufen im Ausmaß ihres Sortierens in der Lernphase oder im Clustern in der Abrufphase unterscheiden. Es zeigte sich, dass die Sortierer in der Lernphase tendenziell mehr Items entsprechend der vorliegenden Kategorien ordneten als die Clusterer in der Lernphase (Abbildung 5.1). Dieser Unterschied war bei bei Sechstklässlern allerdings nur noch sehr gering. 


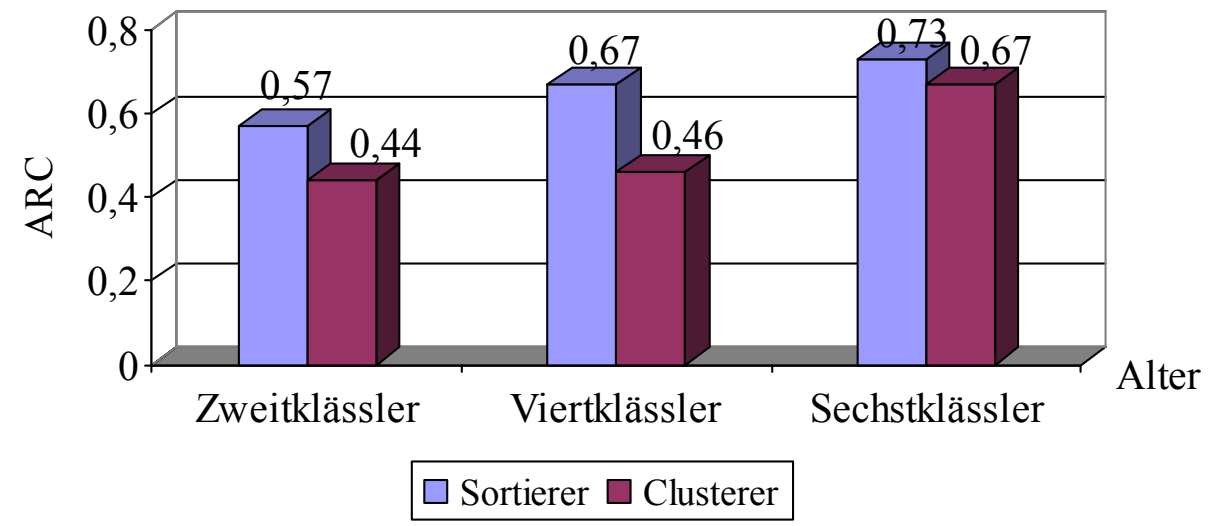

Abbildung 5.1: Mittleres Ausmaß kategorialer Organisation getrennt nach Lern- und Abrufphase bei kategorial organisierenden Kindern

Zur Beantwortung der zuvor formulierten Frage, ob kategoriales Organisieren bei älteren Kindern effektiver abläuft als bei jüngeren, wurden die Reproduktionsleistungen von Kindern aus unterschiedlichen Altersstufen, die in der „Sort-Recall“-Aufgabe in vergleichbarem Ausmaß kategorial organisierten, miteinander verglichen. Es zeigte sich, dass in allen Altersstufen die Sortierer mehr Items erinnerten als die Clusterer. Die Höhe der Reproduktionsleistung der kategorialen Organisierer, in Abhängigkeit von der Phase, in der kategorial organisiert wird, und der Altersstufe, kann Abbildung 5.2 entnommen werden.

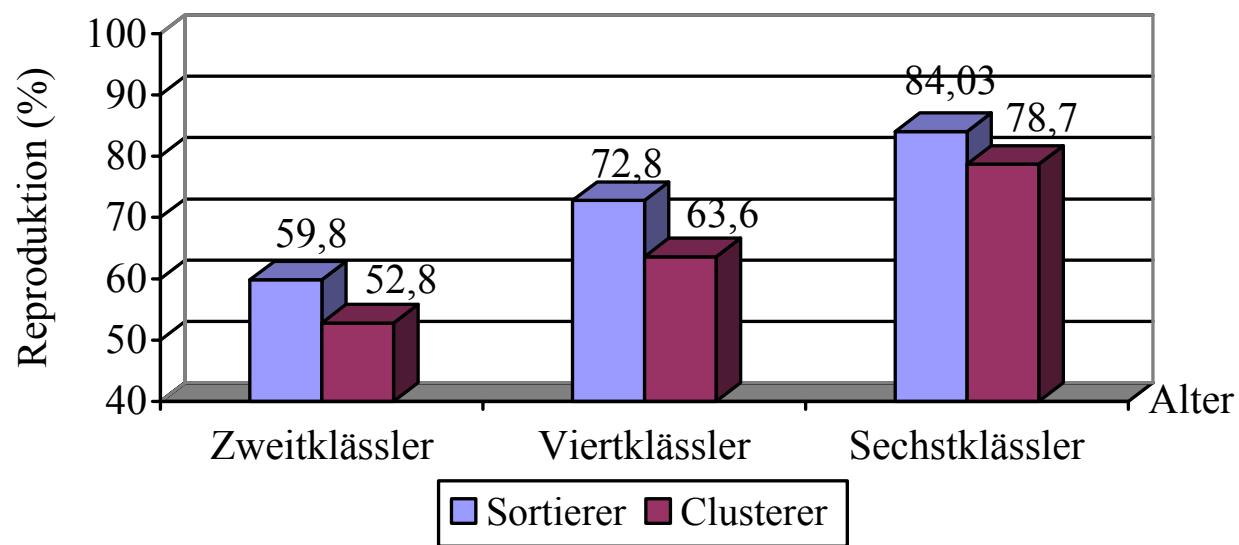

Abbildung 5.2: Mittlere Reproduktionsleistung (in Prozent) getrennt nach kategorial organisierenden Kindern in Lern- vs. Abrufphase

Die statistische Überprüfung dieser Unterschiede mit Hilfe einer einfaktoriellen Varianzanalyse mit dem interindividuell bedingungsvariierten Faktor Klassenstufe ergaben für die kategorial organisierenden Kinder in der Lernphase (Sortierer) keinen Haupteffekte für das Sortierverhalten $(F(2,68)=1,75, p<\alpha)$, aber für die Reproduktionsleistung $(F(2,68)=15,93$, 
$\mathrm{p}<\alpha$ ). Für die Gruppe der kategorial organisierenden Kinder in der Abrufphase (Clusterer) zeigte sich hingegen sowohl ein Haupteffekte für das Clustern $(F(2,136)=7,73, p<\alpha)$ als auch für die Reproduktionsleistung $(\mathrm{F}(2,136)=32,99, \mathrm{p}<\alpha)$.

Anschließende Mittelwertsvergleiche ergaben, dass Zweit- und Viertklässler sich sowohl in der Gruppe der Sortierer $(\mathrm{t}(38)=2,41, \alpha<\mathrm{p})$ als auch in der Gruppe der Clusterer $(\mathrm{t}(88)=3,17, \mathrm{p}<\alpha)$ nicht in ihrem Verhalten, sondern nur in ihrer Reproduktionsleistung voneinander unterscheiden. Damit zeigen Zweitklässler gegenüber Viertklässlern das Phänomen des Nutzungsdefizits.

Für eine entsprechende Bewertung der Effektivität des Verhaltens der Grundschulkinder im Vergleich zu den Sechstklässlern wurde zwischen kategorialen Organisierern in der Lern- und in der Abrufphase differenziert. In der Gruppe der Sortierer waren keine altersbezogenen Unterschiede im Ausmaß der kategorialen Organisation zu beobachten $(\mathrm{p}>\alpha)$, aber in der Höhe der Reproduktionsleistung $\left(\mathrm{t}(51)_{\text {Vgl. zu Zweitklässern }}=5,89\right.$, $\left.\mathrm{p}<\alpha ; \mathrm{t}(47)_{\text {Vgl. zu Viertklässern }}=2,57, \alpha=.01\right)$. Eine analoge Aussage für die Gruppe der kategorialen Organisierer in der Abrufphase war hingegen nicht möglich, da die Sechstklässler im Vergleich $\mathrm{zu}$ den Grundschülern sowohl bessere Erinnerungsleistungen $\left(\mathrm{t}(102)_{\mathrm{Vgl} \text {. zu }}\right.$ Zweitklässern $=8,48, p<\alpha ; t(82)$ Vgl. zu Viertklässern $=3,9, p<\alpha)$ als auch ausgeprägtere Clusterleistungen $\left(\mathrm{t}(102)_{\text {Vgl. zu Zweitklässern }}=3,73, \mathrm{p}<\alpha ; \mathrm{t}(82)_{\text {Vgl. zu Viertklässern }}=2,79, \alpha=.01\right)$ zeigten.

\section{Vertrautheit mit den Anforderungen}

Darüber hinaus interessierte die Frage, ob die Vertrautheit mit den Anforderungen bzw. die Beschaffenheit des Lernmaterials die Effektivität des kategorialen Organisierens beeinflusst. Aus Gründen der Übersichtlichkeit beschränkt sich die folgende Darstellung auf Befunde, die von denen in Liste 1 gefundenen abweichen und daher eine differenzierte Sichtweise erfordern. Um diese Befunde von dem Befundmuster des spontanen Strategiegebrauchs abzugrenzen, werden Befunde der zweiten Liste eingerückt dargestellt.

\section{Besonderheiten unter vertrauten Anforderungen (bei hoher kategorialer Typizität der Items)}

Unter vertrauten Bedingungen (mit kategorial hoch-typischen Items) konnte in folgenden Altersstufen ein Nutzungsdefizit im Sinne einer unterschiedlichen Reproduktionsleistung bei gleichem Verhalten beobachtet werden: 
- Zweitklässler wiesen gegenüber Viertklässlern ein Nutzungsdefizit beim

kategorialen Organisieren in der Abrufphase auf $(\mathrm{t}(99)=3,24, \alpha=.002)$

- Z Zweit- und Viertklässler ließen gegenüber Sechstklässlern ein Nutzungsdefizit beim kategorialen Organisieren in der Lernphase erkennen $\left(\mathrm{t}(64)_{\mathrm{Vgl} . \mathrm{zu}}\right.$

Zweitklässern $\left.=6,31, \alpha<\mathrm{p} ; \mathrm{t}(31)_{\mathrm{Vgl.} \mathrm{zu} \text { Viertklässern }}=3,55, \alpha=.01\right)$.

\section{Besonderheiten unter vertrauten Anforderungen (bei erhöhter Itemanzahl)}

Unter vertrauten Bedingungen (mit einer erhöhten Itemanzahl) konnte in folgenden Altersstufen ein Nutzungsdefizit beobachtet werden:

- Zweitklässler hatten gegenüber Viertklässlern ein Nutzungsdefizit beim kategorialen Organisieren in der Lern- $(\mathrm{t}(21)=2,4, \alpha=.03)$ und Abrufphase $(\mathrm{t}(47)=3,48, \alpha=.001)$

- Viertklässler wiesen gegenüber Sechstklässlern ein Nutzungsdefizit beim kategorialen Organisieren in der Lernphase auf $(\mathrm{t}(33)=3,07, \alpha=.004)$.

\section{Altersveränderungen (Längsschnittliche Analysen)}

Erwartet wurde, dass mit zunehmendem Alter die kategorialen Strukturen bei der Bearbeitung der Sort-Recall-Aufgabe stärker genutzt werden. Das vermehrte kategoriale Organisieren sollte mit einer Leistungssteigerung einhergehen, d.h. effektiv sein.

Da die Interpretation negativer Werte Schwierigkeiten bereitet (vgl. Abschnitt 4.2.1) und der Fokus auf der Verbesserung des strategischen (und nicht des unstrategischen) Verhaltens liegt, gingen in die folgende Auswertung - wie bereits bei der Untersuchung der Altersunterschiede - nur Werte von Kindern ein, die kategoriales Organisationsverhalten zeigten, d.h. positive ARC-Werte aufwiesen. Wie Tabelle 5.2. zu entnehmen ist, stieg bis zur vierten Klasse der Anteil der Kinder an, die kategoriale Ordnungskriterien beim Lernen oder Erinnern des Lernmaterials nutzten. Während der Anteil der Kinder, die diese Strategie in der Abrufphase nutzten, aber kontinuierlich bis zum Ende der Grundschulzeit zunahm, gab es hinsichtlich der Nutzung der Kategorisierungsstrategie in der Lernphase einen deutlichen Anstieg von der zweiten zur vierten Klasse und einen Rückgang zum Ende der vierten Klasse. 
Tabelle 5.2: Häufigkeitsverteilung der kategorial organisierenden Kinder in Abhängigkeit von der Phase der Aufgabenbearbeitung und dem Messzeitpunkt (Liste 1)

\begin{tabular}{|c|c|c|c|c|c|}
\hline & Ende 2 & Anfang 3 & Ende 3 & Anfang 4 & Ende 4 \\
\hline Lernphase & $\begin{array}{c}\mathrm{N}=22 \\
(25,6 \%)\end{array}$ & $\begin{array}{c}\mathrm{N}=42 \\
(48,8 \%)\end{array}$ & $\begin{array}{c}\mathrm{N}=54 \\
(62,8 \%)\end{array}$ & $\begin{array}{c}\mathrm{N}=69 \\
(80,2 \%)\end{array}$ & $\begin{array}{c}\mathrm{N}=57 \\
(69,5 \%)\end{array}$ \\
\hline Abrufphase & $\mathrm{N}=55$ & $\begin{array}{c}\mathrm{N}=60 \\
(69,8 \%)\end{array}$ & $\begin{array}{c}\mathrm{N}=65 \\
(75,6 \%)\end{array}$ & $\begin{array}{c}\mathrm{N}=71 \\
(82,6 \%)\end{array}$ & $\begin{array}{c}\mathrm{N}=75 \\
(91,5 \%)\end{array}$ \\
\hline
\end{tabular}

Auch das Ausmaß des kategorialen Organisierens verbesserte sich noch bis zum Ende der Grundschulzeit. Am deutlichsten ausgeprägt war der Anstieg vom Ende der zweiten bis zum Anfang der dritten Klasse. Auf eine weitere leichte Verbesserung folgte bis zu Beginn der vierten Klasse ein Plateau. Die Verbesserung zum Ende der vierten Klasse fiel in der Abrufphase mäßig, in der Lernphase hingegen sehr deutlich aus. Die kategorialen Strukturen wurden in der Lernphase stets stärker genutzt als in der Abrufphase. Die mittleren ARCWerte der Kinder sind Abbildung 5.3 zu entnehmen.

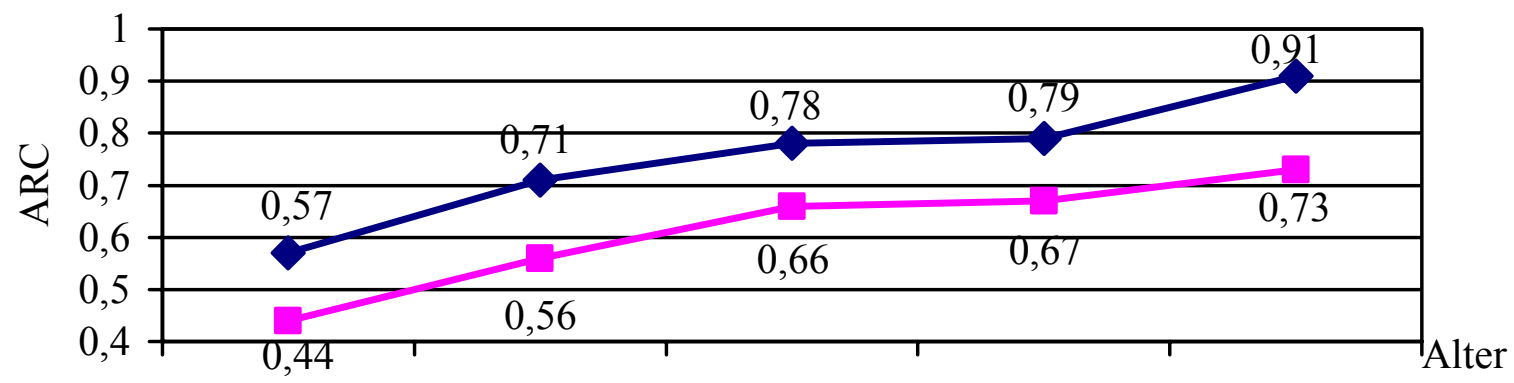

Ende Kl. 2 Anfang Kl. $3 \quad$ Ende Kl. 3 Anfang Kl. 4 Ende Kl. 4

\section{$\neg$ Sortierer $\neg-$ Clusterer}

Abbildung 5.3: Mittleres Ausmaß kategorialer Organisation in Lern- und Abrufphase bei kategorial organisierenden Kindern, getrennt nach Lernphase (Sortierer) und Abrufphase (Clusterer)

Für die Klassifizierung von kategorialem Organisieren als strategisches Verhalten sollten überzufällige positive Interkorrelationen zwischen Sortierleistung, Clustern und Reproduktionsleistung vorliegen (vgl. Frankel \& Rollins, 1985). Die Nutzung der kategorialen Organisationsstrategie in der Lernphase (Sortieren) stand von Anfang an in einem bedeutsamen Zusammenhang mit dem Strategieeinsatz in der Abrufphase (Clustern) und der Wiedergabeleistung (vgl. Tabelle 5.3). 
Tabelle 5.3: Korrelationen zwischen der Reproduktionsleistung und dem Ausmaß kategorialen Organisierens in der Lernphase (Sortierleistung) sowie dem kategorialen Organisieren in der Abrufphase (Clusterleitung) zu den unterschiedlichen Messzeitpunkten

\begin{tabular}{|c|c|c|c|c|c|}
\hline & Ende K1. 2 & Anfang K1. 3 & Ende K1. 3 & Anfang K1. 4 & Ende K1. 4 \\
\hline $\begin{array}{c}\text { Sortierleistung mit } \\
\text { Clusterleitung }\end{array}$ & $.46^{* *}$ & $.76^{* *}$ & $.90^{* *}$ & $.83^{* *}$ & $.82^{* *}$ \\
\hline $\begin{array}{c}\text { Sortierleistung mit } \\
\text { Reproduktionsleistung }\end{array}$ & $.43^{* *}$ & $.62^{* *}$ & $.69^{* *}$ & $.68^{* *}$ & $.70^{* *}$ \\
\hline $\begin{array}{c}\text { Clusterleistung mit } \\
\text { Reproduktionsleistung }\end{array}$ & .07 & $.59^{* *}$ & $.69^{* *}$ & $.66^{* *}$ & $.64^{* *}$ \\
\hline
\end{tabular}

Während die Korrelation am Ende der zweiten Klasse allerdings noch mäßig hoch ausfiel ( $\mathrm{rs} \geq .43$, ps $<\alpha$ ), nahm ab der dritten Klasse der Zusammenhang zur Clusterleistung ( $\mathrm{rs} \geq .76, \mathrm{ps}<\alpha)$ und Reproduktionsleistung ( $\mathrm{rs} \geq .62, \mathrm{ps}<\alpha$ ) deutlich $\mathrm{zu}$. Der Einsatz der kategorialen Organisationsstrategie in der Abrufphase war im Gegensatz zum Sortierverhalten erst $\mathrm{ab}$ der dritten Klasse mit einer entsprechenden Wiedergabeleistung verbunden ( $\mathrm{r} \geq .59$, ps $<\alpha$ ). Im intraindividuellen Entwicklungsverlauf scheint kategoriales Organisieren demnach in der Lernphase ab dem Ende der zweiten Klasse kategoriales Organisieren in der Abrufphase aber erst ab der dritten Klasse leistungsdienlich zu sein.

\section{Besonderheiten unter vertrauten Anforderungen (bei hoher kategorialer Typizität der Items) in der Mitte der Grundschulzeit}

Unter vertrauten Bedingungen (mit kategorial hoch-typischen Items) gab es im Vergleich zur ersten Liste mehr Kinder, die kategorial organisierten (vgl. Tabelle 5.4).

Tabelle 5.4: Häufigkeitsverteilung der kategorial organisierenden Kinder in Abhängigkeit von der Phase der Aufgabenbearbeitung und dem Messzeitpunkt

\begin{tabular}{|c|c|c|c|c|}
\hline \multirow{2}{*}{} & \multicolumn{2}{|c|}{ Ende 2. Klasse } & \multicolumn{2}{c|}{ Anfang 3. Klasse } \\
\cline { 2 - 5 } & Liste 2 & Liste 1 & Liste 2 & Liste 1 \\
\hline Lernphase & $\mathrm{N}=46(53,7 \%)$ & $\mathrm{N}=22(25,6 \%)$ & $\mathrm{N}=65(75,7 \%)$ & $\mathrm{N}=42(48,8 \%)$ \\
\hline Abrufphase & $\mathrm{N}=78(90,7 \%)$ & $\mathrm{N}=55(64 \%)$ & $\mathrm{N}=75(87,2 \%)$ & $\mathrm{N}=60(69,8 \%)$ \\
\hline
\end{tabular}


Besonderheiten unter vertrauten Anforderungen (bei erhöhter Itemanzahl) am Ende der Grundschulzeit

Dieses änderte sich auch nicht, wenn eine höhere Anzahl an Items eingesetzt wurde (vgl. Tabelle 5.5). In der Lernphase ordneten die Kinder durchschnittlich 85-89\%, in der Abrufphase 70-76\% der Items nach Kategorien.

Tabelle 5.5: Häufigkeitsverteilung der kategorial organisierenden Kinder in Abhängigkeit von der Phase der Aufgabenbearbeitung und dem Messzeitpunkt, getrennt für Liste 1 und Liste 2

\begin{tabular}{|c|c|c|c|c|c|c|}
\hline \multirow{2}{*}{} & \multicolumn{2}{|c|}{ Ende Klasse 3 } & \multicolumn{2}{c|}{ Anfang Klasse 4 } & \multicolumn{2}{c|}{ Ende Klasse 4 } \\
\cline { 2 - 7 } & Liste 2 & Liste 1 & Liste 2 & Liste 1 & Liste 2 & Liste 1 \\
\hline \multirow{2}{*}{ Lernphase } & $\mathrm{N}=62$ & $\mathrm{~N}=54$ & $\mathrm{~N}=68$ & $\mathrm{~N}=69$ & $\mathrm{~N}=64$ & $\mathrm{~N}=57$ \\
& $(72,1 \%)$ & $(62,8 \%)$ & $(79,1 \%)$ & $(80,2 \%)$ & $(78 \%)$ & $(69,5 \%)$ \\
\hline \multirow{2}{*}{ Abrufphase } & $\mathrm{N}=78$ & $\mathrm{~N}=65$ & $\mathrm{~N}=78$ & $\mathrm{~N}=71$ & $\mathrm{~N}=81$ & $\mathrm{~N}=75$ \\
& $(90,7 \%)$ & $(75,6 \%)$ & $(90,7 \%)$ & $(82,6 \%)$ & $(98,8 \%)$ & $(91,5 \%)$ \\
\hline
\end{tabular}

Die Interkorrelationen zwischen Sortierleistung, Clustern und Reproduktionsleistung fielen für die zweite Liste stets bedeutsam aus $(.50 \geq \mathrm{r} \geq .67, \mathrm{ps}<\alpha)$.

\subsubsection{Ineffektiver bewusster Einsatz der kategorialen Organisationsstrategie}

Da altersbedingte Unterschiede im strategischen Verhalten mit einer Vielzahl anderer alterskorrelierter Unterschiede im kognitiven und motivationalen Bereich konfundiert sind, sind Unterschiede im individuellen Entwicklungsverlauf aussagekräftiger. Es stellte sich daher die Frage, wie häufig das Phänomen des Nutzungsdefizits im intraindividuellen Verlauf ist. Um Aussagen über strategische Kompetenzen treffen zu können, ist eine Analyse des Verhaltens in allen Phasen der Aufgabenbearbeitung sowie der Bewusstheit des gezeigten Verhaltens erforderlich. Die Erhebung des metamemorialen Bewusstseins der Kinder war notwendig, um sicherzustellen, dass das gezeigte strategische Verhalten von den Kindern nicht aufgrund von automatischen Prozessen zustande kam. Ein vollständiger Strategieeinsatz würde sich darin äußern, dass die Kinder sowohl beim Lernen der Items als auch beim Erinnern der Items bewusst auf die kategorialen Strukturen zurückgreifen, um dadurch ihre Behaltensleistung zu verbessern. 
Anhand der qualitativen Auswertung der Begründungen, die die Kinder für ihr Verhalten im Anschluss an die Bearbeitung der Sort-Recall-Anforderung abgaben, wurde deutlich, dass mit zunehmendem Alter immer mehr Kinder die Strategie des kategorialen Organisierens bewusst einsetzten. Unter Heranziehung eines sehr strengen Kriteriums für metamemoriales Bewusstsein (= spontane Benennung von mindestens zwei verwendeten Kategorien) konnten am Ende der zweiten Klasse nur 4,7\% der Kinder ihr Verhalten richtig begründen. Am Anfang der 3. Klasse erhöhte sich dieser Anteil auf 26,7\% und steigerte sich im Laufe der Grundschulzeit noch weiter (40,7\% am Ende der 3. Klasse, 53,5\% am Anfang und 59,8\% am Ende der vierten Klasse). Um die Bedeutung verbaler Kompetenzen weniger stark ins Gewicht fallen zu lassen, wurde auch den Kindern ein metamememoriales Bewusstsein zugesprochen, die ihre guten Erinnerungsleistungen in der Nachbefragung explizit auf das Kategorisieren des Lernmaterials zurückführten (inkl. der Benennung mindestens einer verwendeten Kategorie). Diese Begründungsstruktur ließen am Ende der zweiten Klasse bereits 15,1\% der Kinder erkennen. In der dritten Klasse stieg der Anteil noch weiter von 38,4\% auf 47,7\% und lag am Anfang der vierten Klasse bei 54,7\% und am Ende der vierten Klasse bei 63,6\%.

Ein Kind sollte erst dann als strategisch bezeichnet werden, wenn es neben der 1.) überzufälligen kategorialen Input-Organisation auch 2.) ein überzufälliges Ausmaß an Output-Organisation und 3.) ein metamemoriales Bewusstsein für die kategoriale Ordnungsstruktur des Lernmaterials zeigt (vgl. Hasselhorn, 1996). Einen Überblick über die Häufigkeitsverteilung der Kinder, die diese Kriterien erfüllten und damit als Strategen klassifiziert werden konnten, liefert Tabelle 5.6.

Tabelle 5.6: Häufigkeitsverteilung von Strategen in Abhängigkeit vom Alter

\begin{tabular}{|c|c|c|}
\hline & Anzahl $(\mathrm{N})$ & Prozentualer Anteil an der Gesamtstichprobe \\
\hline Ende K1. 2 & 9 & $10,5 \%$ \\
\hline Anfang K1. 3 & 25 & $29,1 \%$ \\
\hline Ende K1. 3 & 38 & $44,2 \%$ \\
\hline Anfang K1. 4 & 45 & $52,3 \%$ \\
\hline Ende K1. 4 & 44 & $51,2 \%$ \\
\hline
\end{tabular}

Es zeigte sich, dass im Laufe der Grundschulzeit immer mehr Kinder als Strategen klassifiziert werden konnten. Während am Ende der zweiten Klasse der Prozentsatz noch bei 10,5\% lag, stieg er in der dritten Klasse deutlich an (29,1 \% zu Beginn, 44,2\% am Ende). In 
der vierten Klasse verhielt sich über die Hälfte der Kinder strategisch (52,3\% zu Beginn, $51,2 \%$ am Ende).

Eine differenziertere Betrachtung der Daten zeigte, dass es unter den Strategen immer mehr Kinder gab, die die Strategie perfekt produzierten. Während am Ende der zweiten Klasse nur zwei der strategischen Kinder die zu lernenden Items perfekt sortierten (22,2\%), waren es zu Beginn der dritten Klasse bereits 9 Kinder (36\%). Ab dem Ende der dritten Klasse waren mehr als die Hälfte der Strategen beim Einsatz der kategorialen Organisationsstrategie perfekt (60,5\% - 68,9\%).

Aus den überzufälligen positiven Interkorrelationen zwischen Sortierleistung, Clustern und Reproduktionsleistung lässt sich allerdings nicht schlussfolgern, dass das beobachtete kategoriale Organisieren bei allen Strategen leistungsdienlich ist. Von einer effektiven Strategieanwendung wurde in dieser Arbeit ausgegangen, wenn die Behaltensleistung der Strategen mindestens eine halbe Standardabweichung über der Reproduktionsleistung der Nicht-Strategen lag. Abbildung 5.4 veranschaulicht, dass die Reproduktionsleistungen von Nichtstrategen durchgängig auf einem niedrigeren Niveau lagen als bei Strategen, wobei die Niveauunterschiede relativ konstant blieben.

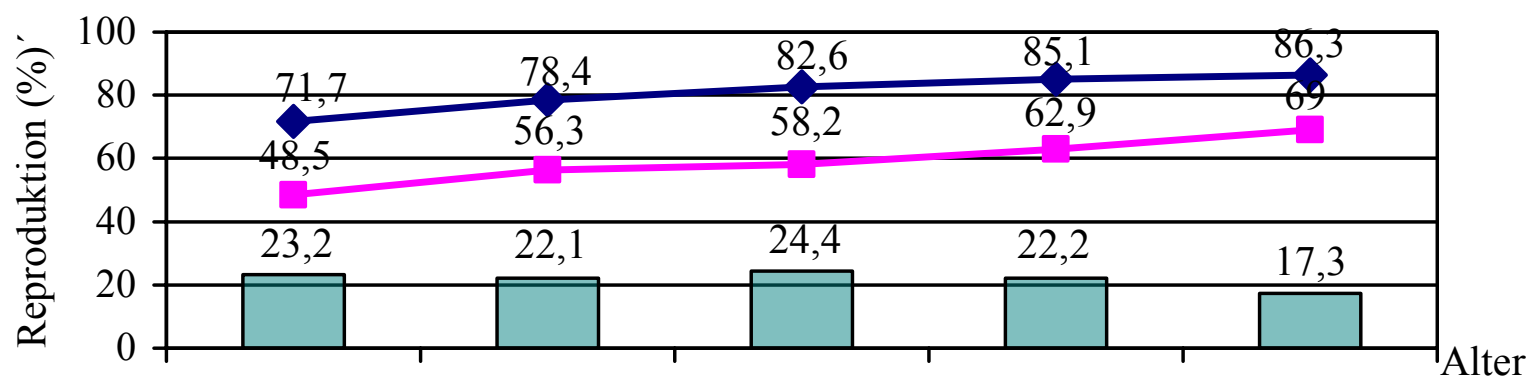

Ende Kl. 2 Anfang Kl. 3 Ende Kl. 3 Anfang Kl. 4 Ende Kl. 4

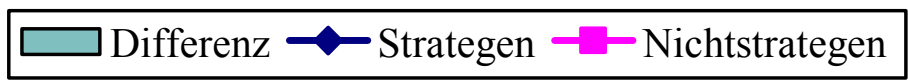

Abbildung 5.4: Prozentuale Reproduktionsleistung der Strategen und Nichtstrategen in Abhängigkeit von den verschiedenen Messzeitpunkten

Im Zusammenhang mit der Entwicklung der kategorialen Organisationsstrategie ergaben sich spezifische Fragestellungen hinsichtlich der Leistungsdienlichkeit des Strategiegebrauchs. Dabei ging es darum zu schauen, ob der Nutzen von Strategien für die Kinder, die sie einsetzen, in verschiedenen Altersabschnitten unterschiedlich ausfällt.

Abbildung 5.5 veranschaulicht, wie weit die Güte des strategischen Verhaltens und die Behaltensleistung in den jeweiligen Altersstufen vom Optimum, d.h. perfekte kategoriale 
Organisation und Reproduktion aller Items, entfernt waren. Sowohl das strategische Verhalten als auch die Erinnerungsleistung lagen stets auf einem sehr hohen Niveau. Das Ausmaß kategorialer Organisation in der Lernphase übertraf dabei die Höhe des in der Abrufphase gezeigten Strategiegebrauchs und der Reproduktionsleistung. Die Strategen sortierten zwischen 79\% und 92\% der Karten nach den Kategorien in der Lernphase. In der Abrufphase wendeten sie die Strategie auf 67\%-83\% der wiedergegebenen Items an und erinnerten zwischen $72 \%$ und $86 \%$ des gesamten Lernmaterials. Ab dem Ende der dritten Klasse blieben die Leistungen auf einem Niveau, d.h. es waren keine weiteren Leistungssteigerungen hinsichtlich des strategischen Verhaltens oder der Erinnerungsleistung zu beobachten.

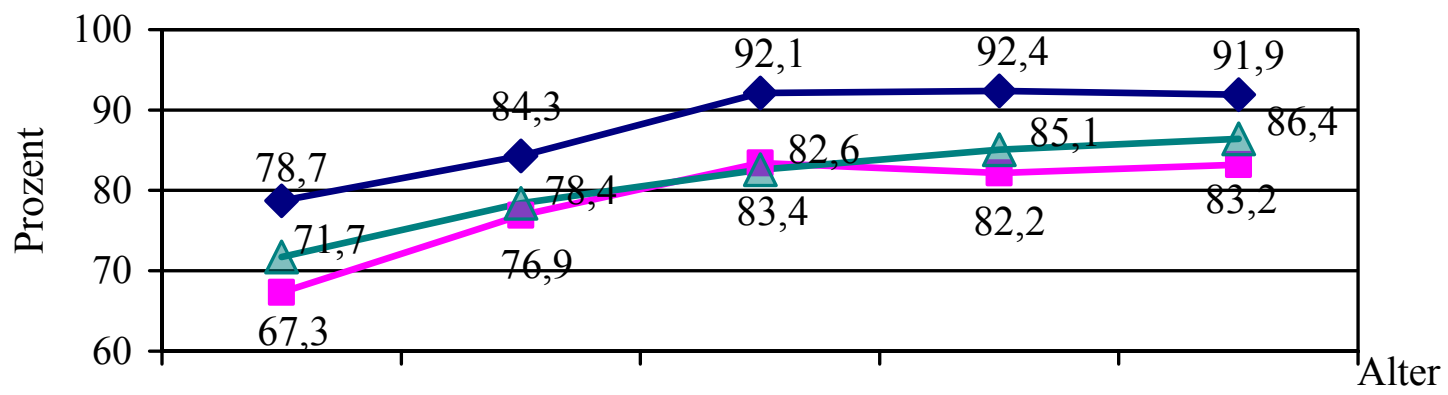

Ende Kl. 2 Anfang Kl. 3 Ende K1. 3 Anfang Kl. 4 Ende Kl. 4

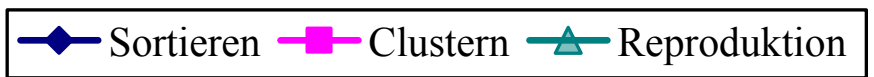

Abbildung 5.5: Prozentuale kategoriale Organisation und Reproduktionsleistung der Strategen in Abhängigkeit von den verschiedenen Messzeitpunkten

Bei effektiven Strategen sollte die Reproduktionsleistung bedeutsam höher als bei Nichtstrategen zum gleichen Zeitpunkt sein. War die Leistung mindestens eine ganze Standardabweichung höher, so wurden die Kinder als effektive Strategen bezeichnet. Strategen, die diese Kriterien nicht erfüllten, wurde ein zugrunde liegendes Nutzungsdefizit unterstellt. Die Häufigkeitsverteilung der nach diesem Kriterium identifizierten Kinder zu den jeweiligen Messzeitpunkten lässt sich Tabelle 5.7 entnehmen. Wie daraus ersichtlich ist, war der Anteil nutzungsdefizitärer Kinder am Ende der zweiten und am Ende der vierten Klasse am größten. Im Verhältnis dazu war der aus der Strategieproduktion gezogene Nutzen am Ende der dritten Klasse am größten. 
Tabelle 5.7: Häufigkeitsverteilung nutzungsdefizitärer Strategen in Abhängigkeit vom Messzeitpunkt

\begin{tabular}{|c|c|c|c|c|c|}
\hline & Ende K1. 2 & Anfang K1. 3 & Ende K1. 3 & Anfang K1. 4 & Ende K1. 4 \\
& $\mathrm{~N}=9$ & $\mathrm{~N}=25$ & $\mathrm{~N}=38$ & $\mathrm{~N}=45$ & $\mathrm{~N}=45$ \\
\hline Strategen mit & $33,3 \%$ & $28 \%$ & $26,3 \%$ & $28,9 \%$ & $35,6 \%$ \\
Nutzungsdefizit & $\mathrm{N}=3$ & $\mathrm{~N}=7$ & $\mathrm{~N}=10$ & $\mathrm{~N}=13$ & $\mathrm{~N}=16$ \\
\hline
\end{tabular}

Hierbei ist allerdings zu bedenken, dass ab der dritten Klasse unter den Strategen ein Teil der Kinder die Strategie bereits mehrfach eingesetzt hatte, während ein anderer Teil sie zum ersten Mal einsetzte. Dieses Verhältnis kann zu den jeweiligen Messzeitpunkten sehr unterschiedlich ausfallen, insbesondere da einige Kinder die Strategie möglicherweise auch wieder aufgegeben haben. Um zu entscheiden, ob bereits der erstmalige Strategiegebrauch effektiv ist, soll im folgenden Abschnitt die Reproduktionssteigerung der Strategieentdecker zu den verschiedenen Messzeitpunkten untersucht werden.

\section{Nutzungsdefizit beim erstmaligen Strategieeinsatz}

Um Aussagen $\mathrm{zu}$ diesen Annahmen treffen zu können, wurden die Kinder entsprechend ihres bewussten strategischen Verhaltens $\mathrm{zu}$ aufeinanderfolgenden Messzeitpunkten in verschiedene Gruppen eingeteilt. Kinder, die beide Male als bewusste Strategen identifiziert werden konnten, wurden als konsistente Strategen bezeichnet, Kinder, die sich zu keinem der beiden Zeitpunkte strategisch verhielten, als konsistente Nichtstrategen. Als Strategieentdecker wurde ein Kind klassifiziert, wenn es das erste Mal sowohl in der Lern- als auch in der Abrufphase überzufälliges kategoriales Organisieren zeigte, dabei seine Sortierleistung um mindestens eine halbe Standardabweichung steigerte (Differenzkriterium) und in der Nachbefragung eine angemessene Begründung für das gezeigte Verhalten abgeben konnte.

Kinder, die die Strategie wieder aufgaben, erhielten die Bezeichnung „Strategieverlierer“. Wie Tabelle $5.8 \mathrm{zu}$ entnehmen ist, verhielt sich ab der dritten Klasse ein Drittel der Kinder zwischen zwei Messzeitpunkten dauerhaft strategisch. Während hierbei eine steigende Tendenz verzeichnet werden konnte (von 24,4 \% auf 41,5\%), sank der Anteil der Nicht-Strategen in dem gleichen Zeitraum von 58,1\% auf 39,8\%. Die meisten Strategieentdecker gab es zu Beginn der dritten Klasse (20,9\%). Am Ende der dritten und zu Beginn der vierten Klasse entdeckten jeweils 11,6\%-12,8\% der Kinder die Strategie, am Ende der vierten Klasse waren es nur noch 6,1\%. Abweichungen in der Häufigkeitsverteilung der 
pro Messzeitpunkt identifizierten Strategen (vgl. Tabelle 5.7) sind darauf zurückzuführen, dass für die Klassifikation als Strategieentdecker sowohl eine deutliche Verbesserung der kategorialen Organisation in der Lernphase als auch der erstmalige Strategiegebrauch erwartet wurde. Aus diesem Grund tauchen in der Tabelle weder Kinder auf, die ihre strategische Kompetenz „schleichend“ verbessern, noch die „Wiederentdecker“, d.h. die Kinder, die die Strategie vorübergehend aufgaben und später erneut einsetzten.

Tabelle 5.8: Häufigkeitsverteilung der Strategentypen in Abhängigkeit von der Veränderung ihres Strategenstatus zu aufeinanderfolgenden Messzeitpunkten (T)

\begin{tabular}{|c|c|c|c|c|}
\hline $\begin{array}{c}\text { konsistente Nicht- } \\
\text { Strategen }\end{array}$ & $\mathrm{N}=59(68,6 \%)$ & $\mathrm{N}=50(58,1 \%)$ & $\mathrm{N}=36(41,9 \%)$ & $\mathrm{T}=32(39,8 \%)$ \\
\hline Strategie-Verlierer & $\mathrm{N}=3(3,5 \%)$ & $\mathrm{N}=4(4,7 \%)$ & $\mathrm{N}=5(5,8 \%)$ & $\mathrm{N}=11(13,4 \%)$ \\
\hline $\begin{array}{c}\text { Strategie- } \\
(\text { Neu)Entdecker }\end{array}$ & $\mathrm{N}=18(20,9 \%)$ & $\mathrm{N}=10(11,6 \%)$ & $\mathrm{N}=11(12,8 \%)$ & $\mathrm{N}=5(6,1 \%)$ \\
\hline $\begin{array}{c}\text { konsistente } \\
\text { Strategen }\end{array}$ & $\mathrm{N}=6(7,0 \%)$ & $\mathrm{N}=21(24,4 \%)$ & $\mathrm{N}=33(38,4 \%)$ & $\mathrm{N}=34(41,5 \%)$ \\
\hline
\end{tabular}

$\mathrm{Zu}$ allen Messzeitpunkten zeigten sich in Abhängigkeit vom Strategenstatus zu aufeinanderfolgenden Messzeitpunkten bedeutsame Unterschiede hinsichtlich der Reproduktionsleistung (T1-T2: $\mathrm{F}(3,82)_{\mathrm{T} 1}=11,31 ; \mathrm{F}(3,82)_{\mathrm{T} 2}=14,29 ; \mathrm{T} 2-\mathrm{T} 3: \mathrm{F}(3,81)_{\mathrm{T} 2}=14,16$; $\mathrm{F}(3,81)_{\mathrm{T} 3}=13,93 ; \mathrm{T} 3-\mathrm{T} 4: \mathrm{F}(3,81)_{\mathrm{T} 3}=20,51 ; \mathrm{F}(3,81)_{\mathrm{T} 4}=27,36 ; \mathrm{T} 4-\mathrm{T} 5: \mathrm{F}(3,78)_{\mathrm{T} 4}=20,82 ;$ $\left.\mathrm{F}(3,78)_{\mathrm{T} 5}=7,69, \mathrm{ps}<\alpha\right)$.

Weiterhin soll überprüft werden, ob die Entdecker der kategorialen Organisationsstrategie bei ihrem ersten Strategiegebrauch bereits von der kategorialen Organisationsstrategie profitieren. Vergleicht man die Reproduktionsleistung der Strategieentdecker mit der Leistung der NichtStrategen zur selben Zeit, so wird deutlich, dass die Entdecker nach ihrer ersten Strategieanwendung die nichtstrategischen Kinder übertreffen und sich auf einem Niveau mit den dauerhaften Strategen befinden (vgl. Abb. 5.6). 


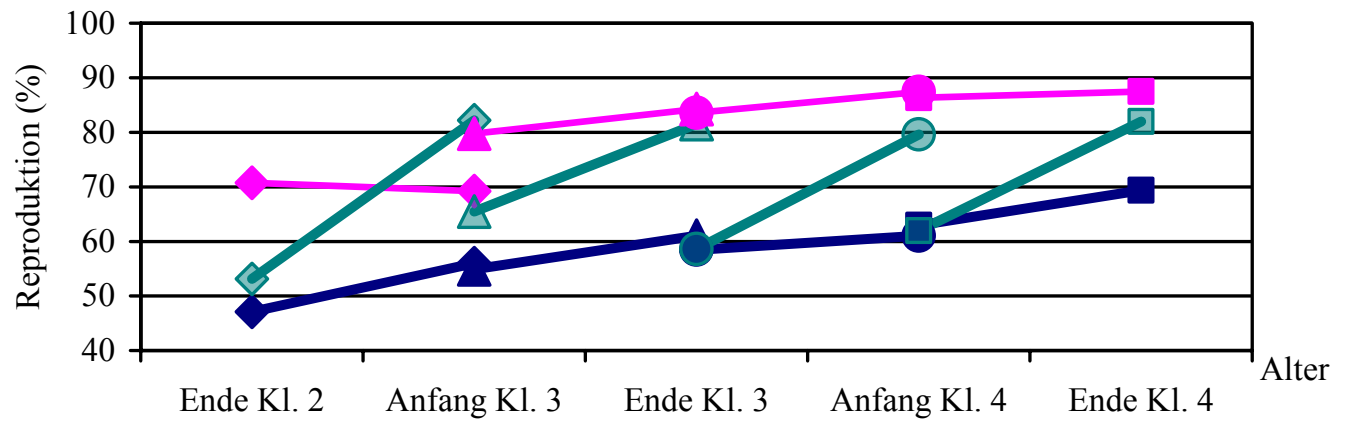

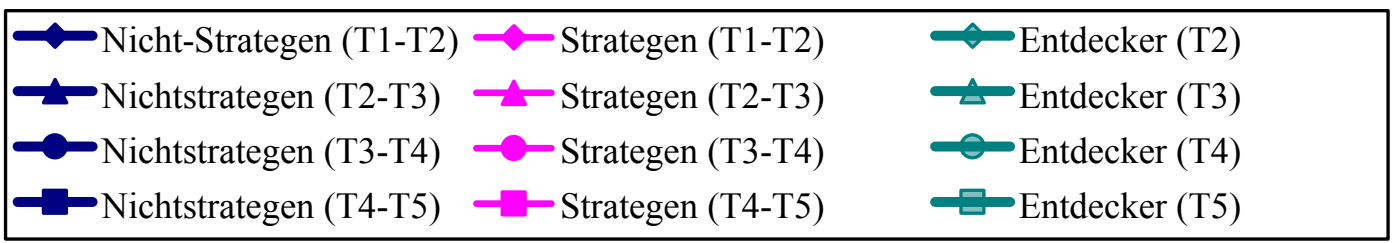

Abbildung 5.6: Prozentuale Reproduktionsleistung in Abhängigkeit vom Strategenstatus zu benachbarten Messzeitpunkten (T), getrennt nach Strategen, Nichtstrategen und Entdeckern

Darüber hinaus interessierte die Frage, ob die Strategieentdecker auch nach der ersten Strategieproduktion gute Reproduktionsleistungen zeigen. Eine Analyse der späteren Leistungen von Strategieentdeckern und Nicht-Strategen über entsprechende Mittelwertsvergleiche unterstützte diese Annahme bei den „frühen“ Strategieentdeckern. Kinder, die zu Beginn der dritten Klasse ( $t_{\text {Anfang K1. } 3}(75)=6,61 ; t_{\text {Ende K1. } 3}(75)=4,49 ; t_{\text {Anfang K1.4 }}$ $\left.(75)=3,43 ; t_{\text {Ende K1. } 4}(72)=3,29\right)$ oder am Ende der dritten Klasse das erste Mal die Kategorisierungsstrategie einsetzten $\left(\mathrm{t}_{\text {Ende K1. } 3}(58)=3,7 ; \mathrm{t}_{\mathrm{Anfang} \mathrm{K1.4}}(58)=3,71 ; \mathrm{t}_{\text {Ende K1. } 4}(55)=\right.$ $3,1, \mathrm{ps}<\alpha$ ), waren von ihrem erstmaligen Strategieeinsatz an bis zum Ende der Grundschulzeit den Nicht-Strategen in ihrer Reproduktionsleistung überlegen. Entdecker zu Beginn der vierten Klasse erinnerten zumindest noch zum Zeitpunkt ihres ersten Strategieeinsatzes mehr als die Nicht-Strategen in dieser Zeit $(\mathrm{t}(45)=3,84, \mathrm{p}<\alpha)$.

Daraus ergab sich die Frage, ob umgekehrt das Aufgeben der Strategie mit einem Rückgang der Reproduktionsleistung verbunden ist. Ab der vierten Klasse standen ausreichend Strategieverlierer zur Verfügung, um diese Vermutung empirisch zu überprüfen. Erwartungsgemäß hatten Strategieverlierer zu Beginn (t $(36)=2,68, \mathrm{p}<\alpha$ ) und am Ende der vierten Klasse $\left(\mathrm{t}_{\text {Ende Kl. }} 4(43)=2,99, \mathrm{p}<\alpha\right)$ zum Zeitpunkt der Aufgabe der Strategie eine schlechtere Reproduktionsleistung als die konsistenten Strategen.

Ein Vergleich der Veränderung der Reproduktionsleistung zu benachbarten Messzeitpunkten bei den verschiedenen Strategengruppen zeigte bedeutsame Unterschiede für die jeweiligen Gruppen $\left(\mathrm{F}(3,82)_{\text {Reproduktionsdifferenz } \mathrm{T1}-\mathrm{T} 2}=16,96 ; \mathrm{F}(3,81)_{\text {Reproduktionsdifferenz T2- }}\right.$ 
$\left.{ }_{\mathrm{T} 3}=4,63 ; \quad \mathrm{F}(3,81)_{\text {Reproduktionsdifferenz }} \mathrm{T}_{\mathrm{T}-\mathrm{T} 4}=6,04 ; \quad \mathrm{F}(3,78)_{\text {Reproduktionsdifferenz }} \quad \mathrm{T}_{4}-\mathrm{T} 5=6,57, \quad \mathrm{ps}<\alpha\right)$. Strategieentdecker verbesserten ihre Leistung am deutlichsten (zwischen 16\% und 29,2\%), während Strategieverlierer sich verschlechterten (Abnahme von $1 \%$ bis $12,5 \%$ ). Alterskorrelierte Leistungsverbesserungen waren auch bei den konsistenten Strategen und Nichtstrategen zu verzeichnen, wenn auch nicht besonders stark ausgeprägt. Nach einem geringfügigen Abfall zu Beginn der dritten Klasse (1,7\%) war bei den konsistenten Strategen noch eine leichte Verbesserung zu verzeichnen, allerdings mit abnehmender Tendenz (von 4,5\% am Ende der dritten Klasse auf 1,2\% am Ende der vierten Klasse). Die Reproduktionssteigerung der Nichtstrategen lag zu aufeinanderfolgenden Messzeitpunkten zwischen 2,6\% und 8,9\% (vgl. Abbildung 5.7).

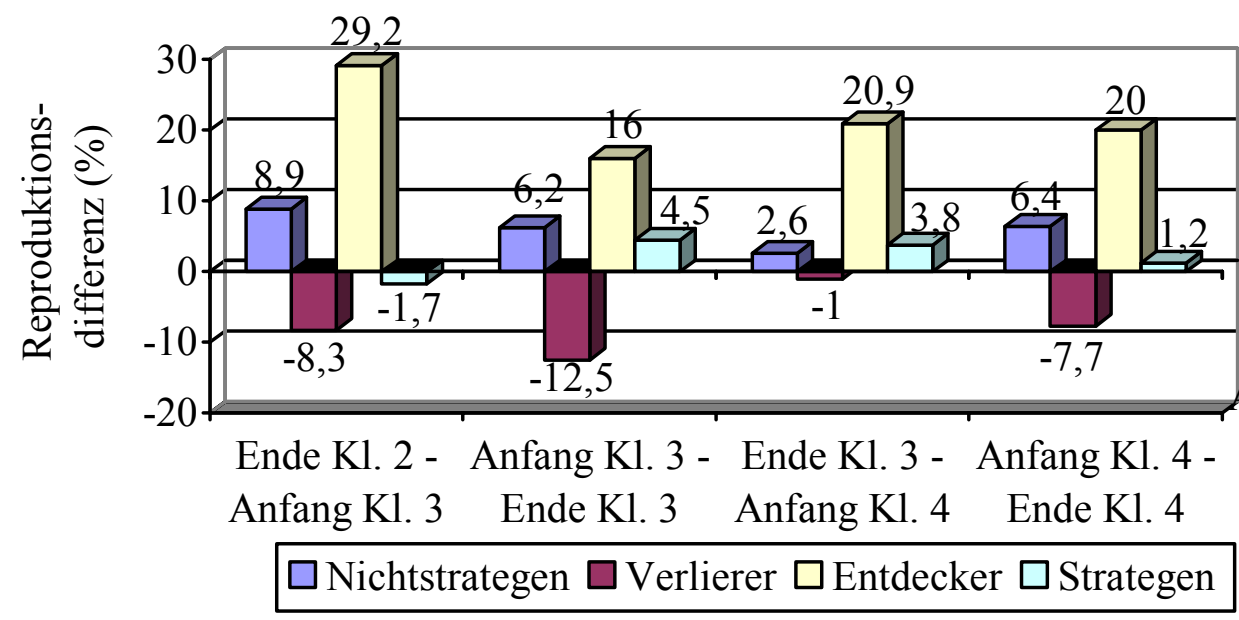

Abbildung 5.7: Veränderungen der Reproduktionsleistung $\mathrm{zu}$ aufeinanderfolgenden Messzeitpunkten in Abhängigkeit vom Strategenstatus

Auf dieser Grundlage ließen sich auch auf Individualniveau nutzungsdefizitäre Kinder identifizieren. Dabei ging es um die Frage, wie viele Kinder von ihrem erstmaligen Strategieeinsatz profitieren und wie viele Strategieentdecker das Phänomen des Nutzungsdefizits zeigen. Als effektive Strategen wurden die Kinder bezeichnet, deren Leistungszuwachs mindestens eine Standardabweichung über der durchschnittlichen Reproduktionssteigerung der nichtstrategischen Kinder lag. Dieses entspricht am Anfang der dritten Klasse $(\mathrm{SD}=4.4)$ und am Ende der vierten Klasse $(\mathrm{SD}=4.3)$ einer Steigerung von mindestens 5 Items und am Ende der dritten $(\mathrm{SD}=3.9)$ sowie zu Beginn der vierten Klasse $(\mathrm{SD}=3.5)$ einer Steigerung von mindestens 4 Items. 
Wie Tabelle 5.9 zu entnehmen ist, gab es unter den Strategieentdeckern zu Beginn der dritten Klasse den größten Anteil an nutzungsdefizitären Kindern (70\%). Aber auch von den Strategieentdeckern am Ende der vierten Klasse profitierten die meisten Kinder (60\%) nicht von ihrem Strategieeinsatz. Demgegenüber war der Anteil der Kinder ohne Leistungssteigerung infolge der Strategieproduktion zu Beginn der dritten und zu Beginn der vierten Klasse eher gering (27,8\% bzw. 36,4\%).

Tabelle 5.9: Häufigkeitsverteilung der Strategieentdecker in Abhängigkeit von der Effektivität der Strategieproduktion zu den jeweiligen Messzeitpunkten

\begin{tabular}{|c|c|c|c|c|}
\hline & Anfang K1. 3 & Ende K1. 3 & Anfang K1. 4 & Ende K1. 4 \\
\hline \multirow{2}{*}{ Effektive Entdecker } & $\mathrm{N}=13$ & $\mathrm{~N}=3$ & $\mathrm{~N}=7$ & $\mathrm{~N}=2$ \\
& $72,2 \%$ & $30 \%$ & $63,6 \%$ & $40 \%$ \\
\hline \multirow{2}{*}{ ineffektive Entdecker } & $\mathrm{N}=5$ & $\mathrm{~N}=7$ & $\mathrm{~N}=4$ & $\mathrm{~N}=3$ \\
& $27,8 \%$ & $70,0 \%$ & $36,4 \%$ & $60 \%$ \\
\hline
\end{tabular}

\section{Besonderheiten unter vertrauten Anforderungen (bei hoher kategorialer}

\section{Typizität der Items) in der Mitte der Grundschulzeit}

Unter vertrauteren Bedingungen ließen sich mehr bewusste Strategen identifizieren als in der ersten Liste (vgl. Abb. 5.8).

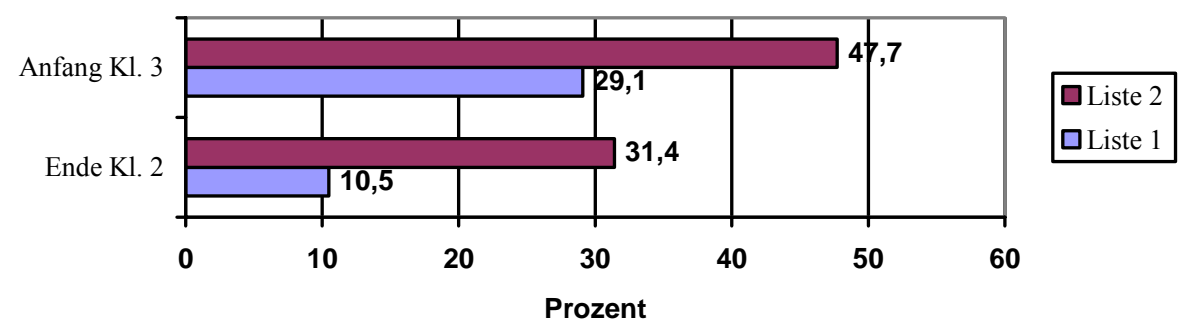

Abbildung: 5.8: Häufigkeitsverteilung der Strategen in Abhängigkeit von Messzeitpunkt und Liste

Unter diesen Strategen gab es zu Beginn der dritten Klasse weniger Kinder mit einem Nutzungsdefizit (14,6\%) als in der ersten Liste (28\%).

Im Vergleich zur ersten Liste setzten weniger Kinder das erste Mal zu Beginn der dritten Klasse bewusst die kategoriale Organisationsstrategie ein (vgl. Abb. 5.9), der prozentuale Anteil an nutzungsdefizitären Kindern war allerdings gleich $(28,6 \%)$. 


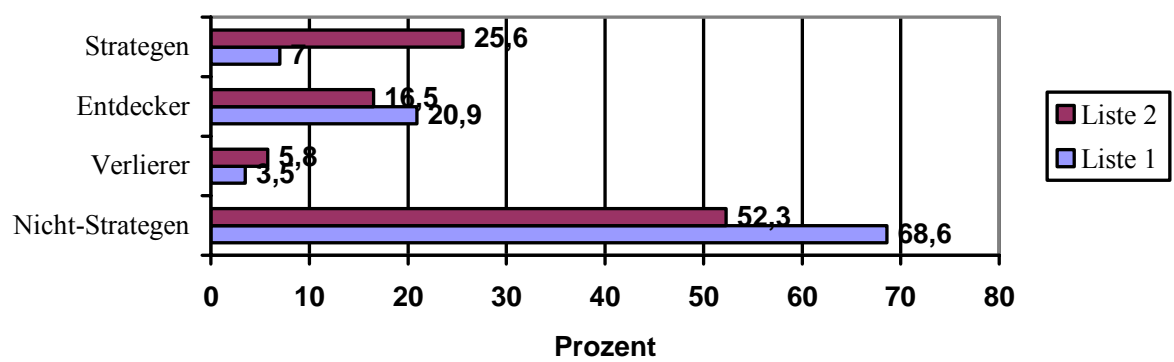

Abbildung 5.9: Häufigkeitsverteilung der Strategen in Abhängigkeit von der Konsistenz der Strategieproduktion zu aufeinderfolgenden Messzeitpunkten (vom Ende der zweiten zur dritten Klasse), getrennt nach Listen

Besonderheiten unter vertrauten Anforderungen (bei erhöhter Itemanzahl) am Ende der Grundschulzeit

Der Anteil der Kinder, die ab dem Ende der dritten Klasse die kategoriale Organisationsstrategie anwendeten, entsprach dem in der ersten Liste (46,5-54,7\%).

Im Vergleich zur ersten Liste war der Anteil der Kinder mit einem Nutzungsdefizit leicht erhöht (vgl. Abb. 5.10). Die größte Diskrepanz gab es dabei zu Beginn der vierten Klasse (45,7\% vs. $28,9 \%)$.

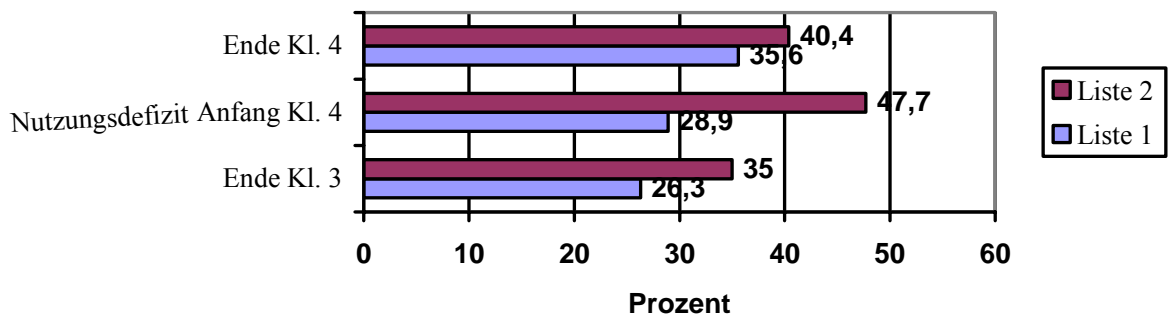

Abbildung 5.10: Häufigkeitsverteilung nutzungsdefizitärer Strategen (prozentualer Anteil an der Gesamtstichprobe, Stichprobengröße in Klammern) in Abhängigkeit vom Messzeitpunkt

$\mathrm{Zu}$ Beginn der vierten Klasse entsprach der Anteil an Strategieentdeckern exakt dem in der ersten Liste $(12,8 \%)$, davon profitierte knapp die Hälfte nicht von dem erstmaligen Strategiegebrauch (45,5\%). Bei Ergänzung einer weiteren Kategorie am Ende der vierten Klasse gab es nur ein Kind, das die Strategie zu diesem Zeitpunkt das erste Mal einsetzte, was auch nicht mit einem Leistungsvorteil verbunden war. 


\section{Geringe Leistungsdienlichkeit der Strategieproduktion in der Anfangsphase}

Ein Hinweis auf das Vorliegen eines Nutzungsdefizits sollte nach Miller und Seier (1994) darin bestehen, dass die Wiedergabeleistung in der Anfangsphase durch den spontanen Strategiegebrauch behindert wird (Ineffektivität der anfänglichen Strategieproduktion). Kinder, die bereits häufig Strategien eingesetzt haben, sollten daher anderen Strategen in ihrer Cluster- und Reproduktionsleistung am Ende der Grundschulzeit überlegen sein.

Um diese Annahme zu überprüfen soll im Folgenden die Leistung am Ende der Grundschulzeit in Abhängigkeit von der Erfahrung mit der Strategieanwendung untersucht werden. Erfahrene und unerfahrene Strategen sollten sich - wenn ein Nutzungsdefizit vorliegt - am Ende der vierten Klasse zwar in der Qualität der Strategieproduktion unterscheiden, aber nicht in ihrer Erinnerungsleistung.

Hinsichtlich der individuellen Entwicklungsverläufe zeigte sich ein sehr heterogenes Muster. Bis zum Ende der Grundschulzeit probierten die meisten Kinder die kategoriale Organisationsstrategie $\mathrm{zu}$ irgendeinem Zeitpunkt aus, taten dieses allerdings mit unterschiedlicher Frequenz (17,1\% einmal; 14,6\% zweimal; 17,1\% dreimal; 18,3\% viermal, $4,9 \%$ immer). Da nur sehr wenige Kinder die Kategorisierungsstrategie seit Beginn der Untersuchung kontinuierlich einsetzten, wurde in einem weiteren Auswertungsschritt die Häufigkeitsverteilung der Strategieproduktion ab Beginn der dritten Klasse bis zum Ende der vierten Klasse untersucht. In diesem Zeitraum setzten 17,1\% der Kinder einmal, 15,9\% zweimal und jeweils 19,5\% drei- oder viermal die kategoriale Organisationsstrategie ein. Der größte Teil der Kinder behielt die Strategie nach der ersten Anwendung konsistent bei, den zweithöchsten Anteil bildeten die „Veränderer“, den geringsten Anteil nahmen die Kinder ein, die die Strategie endgültig aufgaben.

Um zu klären, ob es bedeutsame Unterschiede in dem strategischen Verhalten am Ende der vierten Klasse zwischen den Gruppen gab, wurden univariate Varianzanalysen mit der Nutzungshäufigkeit der Strategie als unabhängiger Variable und dem Ausmaß kategorialen Organisierens sowie der Reproduktionsleistung als abhängigen Variablen durchgeführt. Es zeigte sich, dass sich die Sortierleistung $(F(3,55)=4,25, p<\alpha)$ und die Clusterleistung $(\mathrm{F}(3,55)=9,07, \mathrm{p}<\alpha)$ ebenso wie die Reproduktionsleistung $(\mathrm{F}(3,55)=6,04$, $\mathrm{p}<\alpha)$ in Abhängigkeit von der Häufigkeit der bisherigen Strategieanwendung unterschieden. 


\section{Kontinuierliche Strategieproduktion}

Die Beobachtung, dass Unterschiede in der Strategiequalität nur bei einem sehr deutlichen Erfahrungsunterschied auftraten, könnte allerdings auch darauf zurückzuführen sein, dass einige Kinder die Strategie vorübergehend wieder aufgaben. Ein einheitlicheres Muster könnte bei den Kindern zu erwarten sein, die die kategoriale Organisationsstrategie nach ihrem erstmaligen Gebrauch beibehielten, also kontinuierlich einsetzten (vgl. Tabelle 5.10). Im Gesamtverlauf zeigte sich, dass es ab dem Ende der dritten Klasse deutlich mehr Kinder gab, die die kategoriale Organisationsstrategie entdeckten und beibehielten $(21,9 \%)$ als Kinder, die sie endgültig wieder aufgaben $(9,8 \%)$.

Tabelle 5.10: Häufigkeitsverteilung der Strategen in Abhängigkeit vom Zeitpunkt der erstmaligen Strategieproduktion oder -aufgabe

\begin{tabular}{|c|c|c|c|}
\hline & Zeitpunkt & & \\
\hline \multirow{4}{*}{ Konsistente Strategen } & ab Anfang K1. 3 & $19,5 \%(\mathrm{~N}=16)$ & \\
\hline & ab Ende K1. 3 & $8,5 \%(\mathrm{~N}=7)$ & \multirow{3}{*}{$21,9 \%(\mathrm{~N}=18)$} \\
\hline & ab Anfang K1. 4 & $7,3 \%(\mathrm{~N}=6)$ & \\
\hline & ab Ende K1. 4 & $6,1 \%(\mathrm{~N}=5)$ & \\
\hline \multirow{4}{*}{ Konsistente Nichtstrategen } & ab Anfang Kl. 3 & $30,5 \%(\mathrm{~N}=25)$ & \\
\hline & ab Ende K1. 3 & $3,7 \%(\mathrm{~N}=3)$ & \multirow{3}{*}{$9,8 \%(\mathrm{~N}=8)$} \\
\hline & ab Anfang K1. 4 & $1,2 \%(\mathrm{~N}=1)$ & \\
\hline & ab Ende K1. 4 & $4,9 \%(\mathrm{~N}=4)$ & \\
\hline
\end{tabular}

Der Frage, ob es bedeutsame Unterschiede in dem strategischen Verhalten am Ende der vierten Klasse zwischen den Gruppen gibt, wurde mit Hilfe univariater Varianzanalysen mit der Strategiehäufigkeit als unabhängiger Variable und dem Ausmaß kategorialen Organisierens sowie der Reproduktionsleistung als abhängigen Variablen nachgegangen. Diese erbrachte bedeutsame Unterschiede für die Clusterleistung $(F(3,30)=4,37, p<\alpha)$ und die Reproduktionsleistung $(F(3,30)=6,87, p<\alpha)$, aber nicht für die Sortierleistung $(p>\alpha)$.

Anschließende t-Tests zeigten, dass die Clusterunterschiede darauf zurückzuführen waren, dass die Entdecker zu Beginn der vierten Klasse den Dauerstrategen deutlich unterlegen waren $(\mathrm{t}(20)=3,54, \mathrm{p}<\alpha)$. Reproduktionsunterschiede waren zwischen Entdeckern am Ende der dritten und Entdeckern zu Beginn der vierten Klasse $(\mathrm{t}(11)=2,83, \mathrm{p}<\alpha)$ sowie zwischen Dauerstrategen und Entdeckern zu Beginn $(\mathrm{t}(20)=3,85, \mathrm{p}<\alpha)$ und am Ende der vierten Klasse $(\mathrm{t}(19)=2,63, \mathrm{p}<\alpha) \mathrm{zu}$ beobachten, wobei die früheren Entdecker den späteren 
Entdeckern jeweils überlegen waren. Abbildung 5.11 stellt noch einmal die durchschnittliche Cluster- und Reproduktionsleistung am Ende der vierten Klasse in Abhängigkeit von der Häufigkeit des kontinuierlichen Strategieeinsatzes gegenüber.

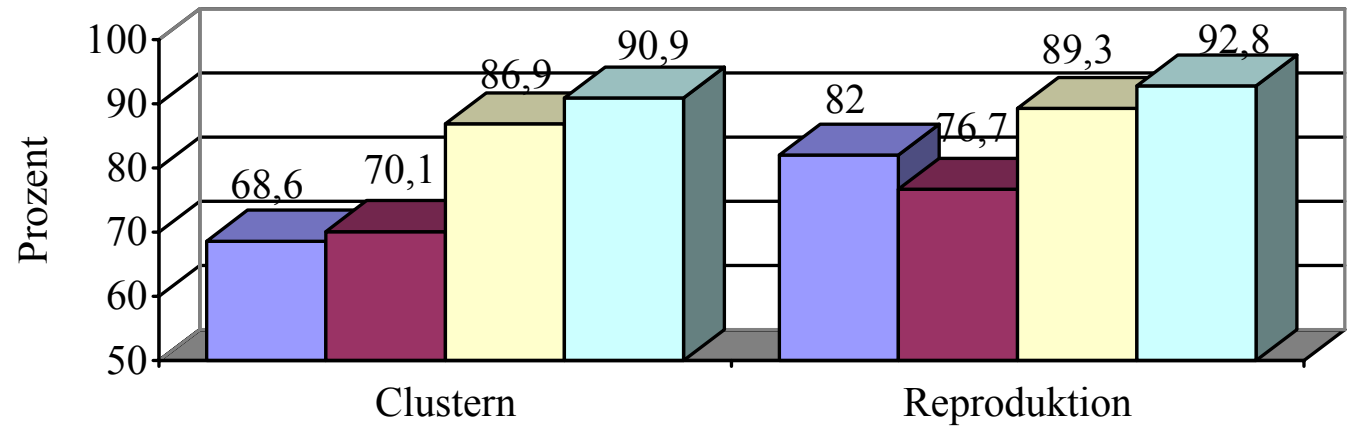

\begin{tabular}{|ll|}
$\square$ Entdecker (Ende K1. 4) & $\square$ Entdecker (Anfang K1. 4) \\
$\square$ Entdecker (Ende K1. 3) & $\square$ Dauerstrategen \\
\hline
\end{tabular}

Abbildung 5.11: Mittlere Sortier-, Cluster- und Reproduktionsleistungen am Ende der vierten Klasse in Abhängigkeit vom Messzeitpunkt, seit dem die Strategie kontinuierlich gezeigt wurde

Kinder, die bereits in der dritten Klasse die kategoriale Organisationsstrategie entdeckten und konsistent einsetzten, schienen den Kindern, die erst seit der vierten Klasse davon Gebrauch machen, in ihrer Leistung überlegen zu sein. Angesichts der Tatsache, dass es keine Unterschiede im strategischen Verhalten in der Lernphase und mit einer Ausnahme auch keine Unterschiede in der Abrufphase gab, könnte dieses im weitesten Sinne als Nutzungsdefizit gedeutet werden. Dieses sollte allerdings sowohl aus inhaltlichen als auch aus methodischen Gründen mit der gebotenen Zurückhaltung geschehen. Einmal lässt sich die „Anfangsphase“ der Strategiegebrauchs nur schwer definieren, darüber hinaus sind nicht alle „frühen“ Strategieentdecker den „späten“ Strategieentdeckern überlegen. Diese explorative Überprüfung der Fragestellung kann demnach nur erste Hinweise auf eine relative Ineffektivität des anfänglichen strategischen Verhaltens liefern.

\subsubsection{Verhaltenskonsistenz über zwei vergleichbare Aufgaben}

Im Folgenden wird der Frage nachgegangen, ob der Gebrauch der kategorialen Organisationsstrategie in dem betrachteten Altersbereich über zwei vergleichbare Anforderungen hinweg stabil ist. Aus diesem Grund wurde die kategoriale Organisationsanforderung auch noch bei einer zweiten Liste mit neuem Lernmaterial gestellt. 
Dieser Schritt geschieht vor dem Hintergrund, dass die Kontextabhängigkeit strategischen Verhaltens in der Vergangenheit häufig als Produktionsdefizit interpretiert wurde. Wenn Kinder eine Strategie nicht produzieren, kann allerdings keine Aussage darüber getroffen werden, ob sie im Fall einer Strategieproduktion ein Nutzungsdefizit gezeigt hätten. Darüber hinaus kann auch bei Kindern, die bereits die Strategie produziert haben, die Effektivität der Strategie in Abhängigkeit von den gewählten Kontextbedingungen variieren.

Die interessierende Frage lautet, ob es Hinweise darauf gibt, dass Kinder eine Strategie wieder aufgeben, weil sie von der Strategieproduktion keinen Leistungsvorteil haben.

Betrachtet man den Verlauf vom Beginn der dritten Klasse bis zum Ende der vierten Klasse, so zeigt sich, dass in beiden Listen knapp ein Drittel der Kinder (30,5\% in der ersten Liste bzw. 25,6\% in der zweiten Liste) zu keiner Zeit die kategoriale Organisationsstrategie einsetzte. Ebenfalls knapp ein Drittel der Kinder lässt sich als frühe Strategen identifizieren, d.h. Kinder, die bis zur dritten Klasse die Strategie erwarben und beibehielten $(26,8 \%$ in der ersten und 29,3\% in der zweiten Liste). Der Anteil der späten konsistenten Strategen ist demgegenüber wesentlich geringer (d.h. 13,4\% in der ersten und 9,8\% in der zweiten Liste).

Die übrigen Kinder zeigten ein sehr heterogenes Muster hinsichtlich ihres Strategiegebrauchs und werden daher im Folgenden als Veränderer bezeichnet.

Während in der ersten Liste nur 4\% der Kinder eine Strategie wieder endgültig aufgaben, taten dies $13,4 \%$ in der zweiten Liste. Die meisten davon gaben die Strategie am Ende der dritten Klasse auf, also zu dem Zeitpunkt, als die kategoriale Typizität derjenigen der ersten Liste angepasst wurde und die Itemanzahl von 20 auf 25 erhöht wurde. Die Veränderung der Anforderungen scheint also den Strategiegebrauch zu beeinflussen.

Um sicherzustellen, dass keine fragilen Phänomene, sondern wirkliche strategische Kompetenzen beobachtet wurden, wurde die Stabilität des gezeigten strategischen Verhaltens über beide Listen analysiert. Es zeigte sich, dass das Sortieren bereits am Ende der zweiten Klasse relativ stabil über beide Listen war $(\mathrm{r}=.41 ; \mathrm{p}<\alpha)$, allerdings nicht das Clustern $(\mathrm{r}=.09$, $\mathrm{p}=.428$ ). Ab der dritten Klasse waren die Maße für die Strategienutzung stabil (Sortieren: rs $\geq .64, \mathrm{ps}<\alpha$; Clustern: $\mathrm{rs} \geq .51, \mathrm{ps}<\alpha$ ). Während die Stabilität des Clusterns auf einem vergleichbaren Niveau blieb, nahm die Stabilität des Sortierens bis zum Ende der Grundschulzeit deutlich zu (am Ende der 4. Klasse: $r \geq .87, \mathrm{p}<\alpha$ ). 
Am Ende der zweiten Klasse korrelierte der in der zweiten Liste gezeigte Strategiegebrauch stärker mit dem Strategieeinsatz zum darauffolgenden Messzeitpunkt als zum selben Messzeitpunkt. Dieses betraf sowohl das Sortieren ( $r=.77$ vs. $r=.41$, ps $<. \alpha$ ) als auch das Clustern $(\mathrm{r}=.43$ vs. $\mathrm{r}=.09, \mathrm{ps}<. \alpha)$. In der folgenden Zeit nahm die Homogenität des strategischen Verhaltens deutlich zu, sowohl im kurzfristigen als auch im mittelfristigen Verlauf. Das kategoriale Verhalten in der Lernphase war dabei stabiler $(r \geq .62$, $p s<. \alpha)$ als in der Abrufphase $(r \geq .34, \mathrm{ps}<. \alpha)$.

Neben der Verhaltensstabilität wurde geprüft, wie konsistent die Zuordnung eines Kindes zu der Gruppe der Strategen oder Nicht-Strategen ist. Im Mittelpunkt stand dabei die Frage, ob sich über beide Aufgaben hinweg die Klassifikation der Kinder aufrechterhalten lässt oder ob das Ausmaß an Unterstützung (d.h. Vertrautheit der Anforderungen, Menge an Lernmaterial) einen wesentlichen Einfluss auf die Identifikation der strategischen Kompetenzen der Kinder hat. In diesem Fall könnte die Klassifikation der Kinder nur unter Verweis auf die Rahmenbedingungen, unter denen es über die notwendigen Kompetenzen verfügt, vorgenommen werden.

$\mathrm{Zu}$ jedem Zeitpunkt gab es eine überzufällige Konsistenz der Strategenzuordnung über die beiden Aufgaben (Listen) hinweg $\left(\Phi_{\text {Ende Kl. } 2}=.505 ; \Phi_{\text {Anfang Kl. } 3}=.671 ; \Phi_{\text {Ende Kl. } 3}=.860\right.$; $\Phi_{\text {Anfang K1. } 4}=.669 ; \Phi_{\text {Ende Kl. } 4}=.654$, ps $<\alpha$ ). Während am Ende der zweiten Klasse noch 9 Strategen in der ersten Liste 27 Strategen in der zweiten Liste gegenüber standen, näherte sich dieses Verhältnis zu den späteren Zeitpunkten deutlich an. Zu Beginn der dritten Klasse verhielten sich 25 Kinder in der ersten und 41 Kinder in der zweiten Liste strategisch. Ab dem Ende der dritten Klasse erwies sich in beiden Listen die Hälfte der Kinder als Strategen, d.h. es traten keine bedeutsamen Unterschiede in Abhängigkeit von der Vertrautheit mit den Aufgabenanforderungen auf.

Bis zum Beginn der dritten Klasse gab es kein Kind, das in der ersten, aber nicht in der zweiten Liste strategisch war. Allerdings wendeten relativ viele Nicht-Strategen der ersten Liste die Strategie in der zweiten Liste an. Ab dem Ende der dritten Klasse gab es auch Kinder, die sich nur in der ersten Liste strategisch verhielten. Dafür gab es insgesamt deutlich weniger Kinder, die ihr Verhalten bei der Aufgabenbearbeitung von der ersten zur zweiten Liste änderten (vgl. Tabelle 5.11). 
Tabelle 5.11: Häufigkeitsverteilung der Strategen in der ersten und zweiten Liste in Abhängigkeit vom Messzeitpunkt (MZP)

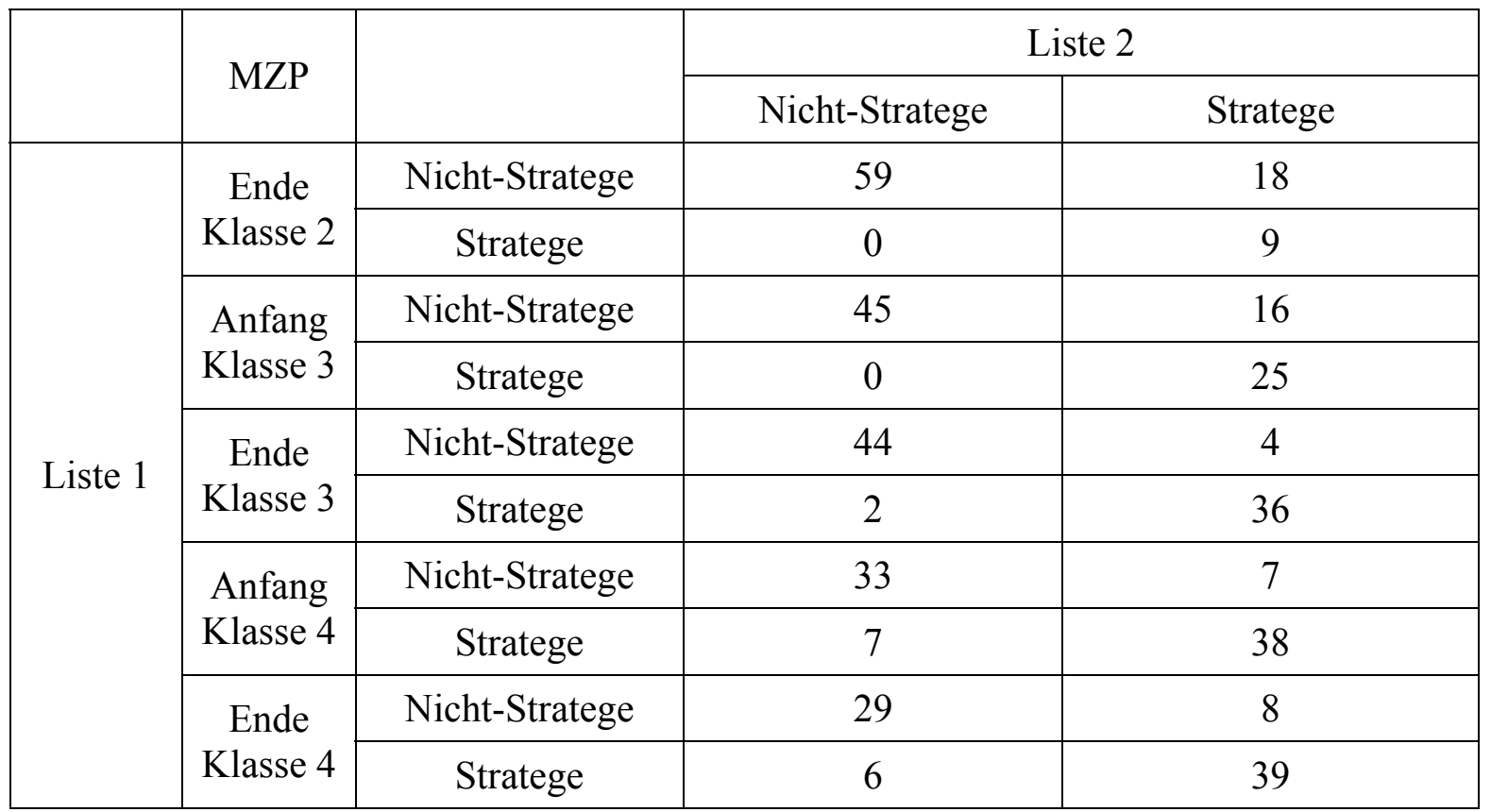

\section{Perfektes kategoriales Organisieren}

Darüber hinaus zeigte sich, dass es Kinder gab, die ohne in der ersten Liste strategisch gewesen zu sein in der zweiten Liste bei ihrem ersten Strategieeinsatz perfekt kategorial organisierten. Von den Nicht-Strategen der ersten Liste zeigten am Ende der zweiten Klasse 16 Kinder (18,6 \%) und Anfang der dritten Klasse 13 Kinder $(15,1 \%)$ eine perfekte bewusste Organisationsstrategie. Ab Ende der dritten Klasse lag dieser Prozentsatz nur noch zwischen 3,5\% und 6,1\% (jeweils 3 Kinder am Ende der dritten und vierten Klasse und 5 Kinder zu Beginn der vierten Klasse). Es gab kaum ein Kind (nur ein Kind am Anfang der dritten und Ende der vierten Klasse sowie zwei Kinder zu Beginn der vierten Klasse), das von einem Nicht-Strategenstatus in der zweiten Liste ein halbes Jahr später spontan in der ersten Liste einen perfekten Strategiegebrauch zeigte.

\section{Veränderungen der Strategieeffektivität vom ersten Durchgang (Liste 1) zum zweiten Durchgang (Liste 2)}

Wenn es sich bei dem Nutzungsdefizit um ein kurzfristigeres Phänomen handelt, so sollten die Leistungsverbesserungen sich bereits zwischen zwei Durchgängen zeigen. Aus diesem Grund wurde der Verlauf zwischen den beiden unterschiedlichen Listen näher beobachtet. Wie Tabelle $5.12 \mathrm{zu}$ entnehmen ist, behielten $\mathrm{zu}$ den ersten beiden Messzeitpunkten alle nutzungsdefizitären Strategen der ersten Liste die Strategie in der 
zweiten Liste bei. Während am Ende der zweiten Klasse allerdings nur ein Kind sein Nutzungsdefizit überwinden konnte, gelang es zu Beginn der dritten Klasse allen Kindern.

Tabelle 5.12: Häufigkeitsverteilung der nutzungsdefizitären Strategen (ND) aus der ersten Liste in Abhängigkeit von ihrer Strategieeffektivität in der zweiten Liste und dem jeweiligen Messzeitpunkt (Ende K1. 2 - Anfang K1. 3)

\begin{tabular}{|c|c|c|c|}
\hline \multirow{2}{*}{} & \multicolumn{3}{|c|}{ In der zweiten Liste } \\
\cline { 2 - 4 } & $\begin{array}{c}\text { Aufgabe der } \\
\text { Strategie }\end{array}$ & $\begin{array}{c}\text { Ineffektiver } \\
\text { Strategiegebrauch }\end{array}$ & $\begin{array}{c}\text { Effektiver } \\
\text { Strategiegebrauch }\end{array}$ \\
\hline ND Ende Klasse 2 $(\mathrm{N}=3)$ & $\mathrm{N}=0$ & $\mathrm{~N}=2$ & $\mathrm{~N}=1$ \\
\hline ND Anfang Klasse $3(\mathrm{~N}=7)$ & $\mathrm{N}=0$ & $\mathrm{~N}=0$ & $\mathrm{~N}=7$ \\
\hline
\end{tabular}

Dieses Muster änderte sich ab dem Ende der dritten Klasse, als die Anzahl an zu lernenden Items erhöht wurde (vgl. Tabelle 5.13). Wesentlich mehr Kindern gelang es nicht, ihr Nutzungsdefizit zu überwinden, einige gaben die Strategie auch wieder auf.

Tabelle 5.13: Häufigkeitsverteilung der nutzungsdefizitären Strategen (ND) aus der ersten Liste in Abhängigkeit von ihrer Strategieeffektivität in der zweiten Liste und dem jeweiligen Messzeitpunkt (Ende Kl. 3- Ende K1. 4)

\begin{tabular}{|c|c|c|c|}
\hline \multirow{2}{*}{} & \multicolumn{3}{|c|}{ In der zweiten Liste } \\
\cline { 2 - 4 } & $\begin{array}{c}\text { Aufgabe der } \\
\text { Strategie }\end{array}$ & $\begin{array}{c}\text { Ineffektiver } \\
\text { Strategiegebrauch }\end{array}$ & $\begin{array}{c}\text { Effektiver } \\
\text { Strategiegebrauch }\end{array}$ \\
\hline ND Ende Klasse 3 $(\mathrm{N}=10)$ & $\mathrm{N}=1$ & $\mathrm{~N}=6$ & $\mathrm{~N}=3$ \\
\hline ND Anfang Klasse 4 $(\mathrm{N}=13)$ & $\mathrm{N}=5$ & $\mathrm{~N}=5$ & $\mathrm{~N}=3$ \\
\hline ND Ende Klasse 4 $(\mathrm{N}=16)$ & $\mathrm{N}=5$ & $\mathrm{~N}=7$ & $\mathrm{~N}=4$ \\
\hline
\end{tabular}

\subsubsection{Zusammenfassung der Ergebnisse zum Phänomen des Nutzungsdefizits}

Bis zum Ende der Grundschulzeit gibt es immer mehr Kinder, die bei der Bearbeitung der Sort-Recall-Anforderung kategorial organisieren. Es zeigen sich allerdings deutliche Unterschiede in Abhängigkeit von der Bearbeitungsphase. Während der Anteil der kategorial organisierenden Kinder in der Lernphase von 25,6\% am Ende der zweiten Klasse auf 80,2\% zu Beginn der vierten Klasse ansteigt und danach wieder auf 69,5\% absinkt, ist in der Abrufphase ein kontinuierlicher Anstieg von 64\% auf 91,5\% zu verzeichnen. 
Vergleicht man die Leistungen von Zweit- und Viertklässlern aus der Querschnittstichprobe zu Beginn der Untersuchung, so zeigt sich, dass die jüngeren Kinder gegenüber den älteren ein Nutzungsdefizit aufweisen. Sie profitieren sowohl in der Lern- als auch in der Abrufphase weniger vom kategorialen Organisieren. Ab der dritten Klasse sind allerdings bedeutsame Zusammenhänge zwischen der Sortierleistung, dem Clustern und der Reproduktionsleistung zu beobachten.

Überprüft man demgegenüber, wie viele Kinder eine bewusste kategoriale Organisationsstrategie einsetzen, so wird deutlich, dass erst in der vierten Klasse die Mehrheit der Kinder als strategisch bezeichnet werden kann. Dieser Anteil war am Ende der zweiten Klasse noch sehr niedrig (10,5\%), hatte aber im Verlauf der dritten Klasse stark zugenommen (von 29,1\% auf 44,2\%). $\mathrm{Zu}$ den verschiedenen Messzeitpunkten gibt es unter den Strategen ca. ein Drittel Kinder, deren Leistungsvorteil im Vergleich zu den Nichtstrategen niedriger als erwartet ausfällt $(26,3 \%-35,6 \%)$.

Betrachtet man, wann die kategoriale Organisationsstratgie das erste Mal zum Einsatz kommt, so zeigt sich, dass die meisten Kinder bereits zu Beginn der dritten Klasse die Strategie entdecken (20,9\%). Innerhalb des nächsten Jahres gibt es noch einmal ähnlich viele Entdecker (11,6\% am Ende der dritten, 12,8\% zu Beginn der vierten Klasse), am Ende der vierten nur noch wenige (6,1\%). Ineffektiv ist dieser erste Strategieeinsatz zu Beginn der dritten und vierten Klasse bei jeweils einem Drittel der Kinder (27,8\% bzw. 36,4\%), am Ende der dritten und der vierten Klasse bei ungefähr zwei Dritteln (70\% bwzw. 60\%).

Hinweise auf eine geringe Leistungsdienlichkeit der Kategorisierungsstrategie in der Anfangsphase lassen sich aus dem Vergleich der Reproduktionsleistungen von unerfahrenen und erfahrenen Strategen am Ende der Grundschulzeit ableiten. Am Ende der vierten Klasse sind Kinder, die die Strategie in der dritten Klasse entdecken und beibehalten, den Kindern, die dies erst in der vierten Klasse tun, tendenziell überlegen.

Ein Nutzungsdefizit beim kategorialen Organisieren kann unter vertrauten Bedingungen bei Zweitklässlern im Vergleich zu Viertklässlern in der Abrufphase, unter Verwendung einer erhöhten Itemanzahl auch in der Lernphase beobachtet werden. Im Vergleich zu Sechstklässlern weisen die Grundschüler (bei höherer Itemanzahl nur die Viertklässler) in der Lernphase ein Nutzungsdefizit auf. Die korrelativen Zusammenhänge zwischen Organisationsverhalten und Reproduktionsleistung sprechen allerdings für eine grundsätzliche Leistungsdienlichlichkeit des Verhaltens. Der Anteil ineffektiver Strategen entspricht am Ende der 2. Klasse dem der 1. Liste (1/3), und ist zu Beginn der 3. Klasse etwas 
niedriger, was mit dem erhöhten Strategenanteil zusammenhängt. Der erste Strategiegebrauch ist bei ähnlich vielen Kindern ineffektiv, wir unter spontanen Bedingungen (28,6\% vs. $27,8 \%)$.

Trotz Verwendung einer höheren Anzahl an zu lernenden Items nimmt das kategoriale Organisieren bei der Aufgabenbearbeitung nicht ab. Auswirkungen gibt es in Bezug auf die relative Nützlichkeit, d.h. es gibt mehr Kinder, bei denen ein Nutzungsdefizit beobachtet werden kann.

Über beide Listen hinweg erweist sich das von den Kindern gezeigte Verhalten als konsistent. Alle Strategen der ersten Liste verhalten sich am Ende der zweiten und zu Beginn der dritten Klasse auch in der zweiten Liste strategisch. Nach Erhöhung der Itemanzahl in der zweiten Liste gibt es mehr Kinder, deren Verhalten in Abhängigkeit von der Liste variiert.

Alle ineffektiven Strategen aus der ersten Liste setzen in der zweiten Liste die Strategie erneut ein (bis zum Beginn der dritten Klasse), was sich am Anfang der dritten Klasse für alle als leistungsdienlich herausstellt. Bei einer höheren Anzahl an Lernitems geben zunehmend mehr Kinder, die in der ersten Liste nicht von dem Strategieeinsatz profitiert haben, die Strategie wieder auf. 


\subsection{Determinanten für effektives kategoriales Organisieren}

Die Fragestellungen zu Determinanten eines effektiven Strategiegebrauchs lauten

- Welche Bedeutung hat das aufgabenspezifische Metagedächtnis alleine oder zusammen mit anderen kognitiven oder motivationalen Faktoren für die Entwicklung des kategorialen Organisierens?

Dabei interessiert unter anderem, ob (und wenn ja warum) strategisches Verhalten auch ohne angemessenes Metagedächtnis gezeigt werden kann. In dem Zusammenhang gilt es auch zu klären, warum umgekehrt Kinder trotz eines angemessenen Metagedächtnisses die kategoriale Organisationsstrategie nicht anwenden.

- Besteht ein Zusammenhang zwischen dem Auftreten eines Nutzungsdefizits und der Entwicklungskonstellation des Metagedächtnisses und/oder der Arbeitsgedächtniskapazität?

Ein Aspekt, der hierbei näher beleuchtet werden soll, ist beispielsweise, ob ein angemessenes Metagedächtnis und eine überdurchschnittliche Gedächtnisspanne (rückwärts) zusammen eine hinreichende Bedingung für die effektive Strategieproduktion darstellen.

- Welche internen Voraussetzungen sind in welchen Altersabschnitten für das Anwenden der Kategorisierungsstrategie und seine Effektivität erforderlich?

Hierbei soll die Bedeutung von aufgabenspezifischem Metagedächtnis und Gedächtnisspanne (vorwärts und rückwärts) sowie von motivationalen Prozessen und leistungsbezogenen Facetten des Selbstkonzeptes für die Entwicklung des kategorialen Organisierens und seine Leistungsdienlichkeit analysiert werden.

- Unter welchen Bedingungen kommt es bei bestimmten Voraussetzungen in bestimmten Altersstufen zum effektiven Einsatz der kategorialen Organisationsstrategie?

Dazu wird ergänzend auf Besonderheiten in der Bedingtheit des strategischen Verhaltens und seiner Effektivität im intraindividuellen Entwicklungsverlauf oder 
hinsichtlich interindividueller Unterschiede eingegangen, die im Zusammenhang mit Aspekten der Vertrautheit mit den Anforderungen oder der Beschaffenheit des Materials (kategoriale Typizität, Itemanzahl) stehen.

Bevor der Einfluss einzelner Faktoren auf die Entwicklung des effektiven kategorialen Organisierens untersucht werden kann, muss überprüft werden, welche allgemeinen Altersunterschiede und -veränderungen in den kognitiven und motivationalen Variablen auftreten.

\subsubsection{Altersunterschiede bei kognitiven und motivationalen Faktoren}

Die im strategischen Verhalten beobachteten Altersunterschiede können auch auf andere altersbedingte Unterschiede zurückgeführt werden. Überprüft werden soll, ob die altersbedingten Unterschiede im kognitiven und motivationalen Bereich wirklich auftreten.

Vergleiche der unterschiedlichen Altersgruppen zeigten Unterschiede in der Gedächtnisspanne, d.h. sowohl beim Zahlennachsprechen vorwärts $(F(1,129)=16,51, p<\alpha)$ als auch rückwärts $(\mathrm{F}(1,129)=18,57, \mathrm{p}<\alpha)$. Auch der Umfang des metakognitiven Wissens differierte zwischen den Altersstufen. $(F(1,129)=23,52, \mathrm{p}<\alpha)$. Darüber hinaus unterschieden sich die Kinder hinsichtlich ihres Selbstkonzepts $(F(1,128)=3,31, p<\alpha)$. Anschließende Mittelwertsvergleiche ergaben, dass sich die Grundschüler in allen kognitiven Variablen von den Sechstklässlern unterschieden. Die älteren Kinder waren in ihrer Gedächtnisspanne (vorwärts und rückwärts) sowohl den Zweitklässlern $(\mathrm{t}(107)=5,64 ; \mathrm{t}(107)=5,78$, ps $<\alpha)$ als auch den Viertklässlern $(\mathrm{t}(44)=2,68 ; \mathrm{t}(44)=3,25, \mathrm{ps}<\alpha)$ überlegen. Außerdem verfügten sie über ein besseres Metagedächtnis $(\mathrm{t}(46,2)=8,33 ; \mathrm{t}(36,4)=2,8, \mathrm{ps}<\alpha)$. Unterschiede hinsichtlich des Selbstkonzepts gab es allerdings nur zwischen Zweit- und Sechstklässlern $(\mathrm{t}(106)=2,12$, $\mathrm{ps}<\alpha)$.

Zwischen den Grundschülern waren nur in zwei Determinanten Abweichungen erkennbar. Die Viertklässler verfügten über eine höhere Gedächtnisspanne vorwärts $(\mathrm{t}(107)=2,24, \quad \mathrm{p}<\alpha)$ und besseres metakognitives Wissen $(\mathrm{t}(107)=3,002, \quad \mathrm{p}<\alpha)$ als die Zweitklässler. 


\section{Altersbedingte Verbesserung des Metagedächtnisses}

Das Metagedächtnis ist für die Entwicklung der effektiven Kategorisierungsstrategie in mehreren Phasen der Strategieproduktion relevant. Es ist verantwortlich für das Kennen bzw. Erkennen der Strategie, die Anpassung der Strategie an die aktuellen Anforderungen der Aufgabe und das Beibehalten der Strategie, z.B. aufgrund der Überzeugung, dass die Strategie beim Behalten des Lernmaterials hilft. Bevor auf den Zusammenhang zur Strategieentwicklung eingegangen wird, soll zuerst die Entwicklung der metakognitiven Kompetenzen selber dargestellt werden. Wie Abbildung 5.12 zeigt verbesserte sich mit zunehmendem Alter das Metagedächtnis, wobei die deutlichsten Veränderungen ab der dritten Klasse beobachtbar waren $(F(4,324)=35,49, \mathrm{p}<\alpha)$.

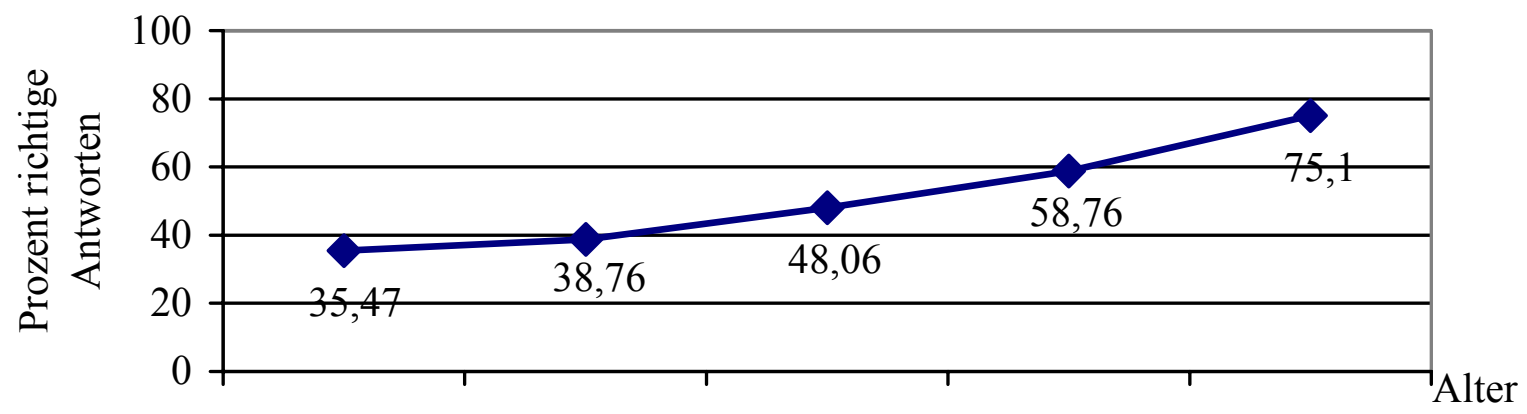

Ende Kl. 2 Anfang Kl. 3 Ende Kl.3 Anfang Kl. 4 Ende Kl. 4

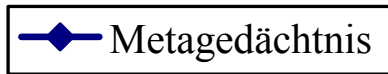

Abbildung 5.12: Durchschnittlicher Anteil richtig beantworteter Items des Metagedächtnisfragebogens in Abhängigkeit vom Alter

Die metakognitiven Kompetenzen stellten sich zwischen den Messzeitpunkten durchgängig als zeitlich stabil heraus (rs $\geq .29$ ).

Um Aussagen darüber treffen zu können, ob und wenn ja wie die oben angeführten Determinanten (Gedächtnisspanne, Motivation und Selbstkonzept) einen Einfluss auf die effektive Strategieproduktion haben können, soll im Folgenden kurz der Entwicklungsverlauf der kognitiven und motivationalen Faktoren skizziert werden.

\section{Altersbedingte Verbesserung der Gedächtnisspanne}

Mit zunehmendem Alter verbesserte sich erwartungsgemäß sowohl die Zahlenspanne vorwärts $(F(4,324)=11,43, p<\alpha)$ als auch rückwärts $(F(4,324)=12,58, p<\alpha)$, wie Abbildung 5.13 zeigt. Aufgrund des erhöhten Schwierigkeitsgrades beim Zahlennachsprechen rückwärts war diese Zahlenspanne geringer als beim Zahlennachsprechen vorwärts. 


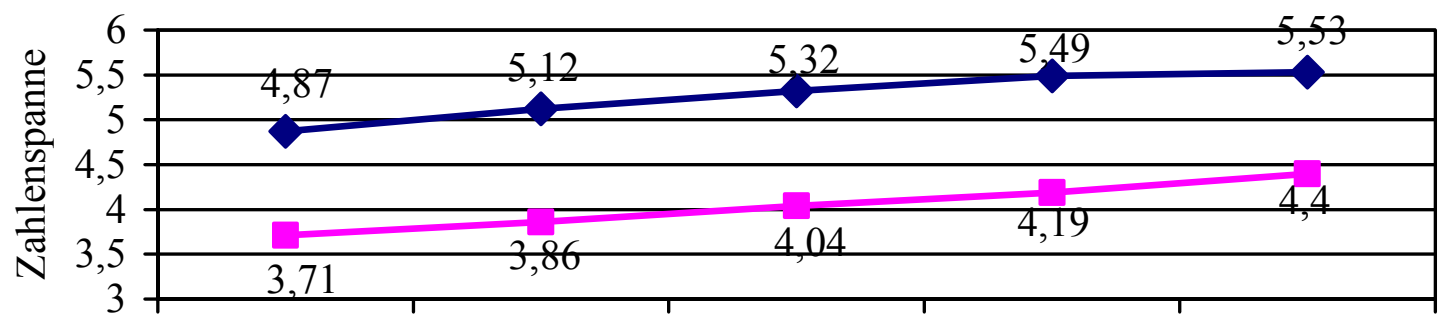

Ende K1. 2 Anfang K1. 3 Ende K1.3 Anfang K1. 4 Ende K1. 4 Alter

$\multimap$ vorwärts $\neg$ rückwärts

Abbildung 5.13: Mittlere Zahlenspanne vorwärts und rückwärts, in Abhängigkeit vom Alter

Die Zahlenspanne (vorwärts) und die Zahlenspanne (rückwärts) waren zwischen den Messzeitpunkten zeitlich stabil ( $\mathrm{rs} \geq .35$ bzw. $\mathrm{rs} \geq .33$, $\mathrm{ps}<\alpha$ ). Ab dem Ende der dritten Klasse bestanden bedeutsame Zusammenhänge zwischen beiden Gedächtnisspannen ( $r s \geq .40, p<\alpha)$.

\section{Konstanz von nicht-kognitiven Faktoren}

Über alle Messzeitpunkt hinweg waren relativ hohe Selbstkonzept- und Motivationswerte $\mathrm{zu}$ verzeichnen, was darauf hinweist, dass die Kinder ihre eigene Leistungsfähigkeit grundsätzlich hoch einschätzten und sich gerne mit Lernanforderungen beschäftigten. Sowohl die Motivation im Umgang mit Lernanforderungen $(F(4,304)=1,46$, $\mathrm{p}=.21)$ als auch das Selbstkonzept $(\mathrm{F}(4,320)=1,697, \mathrm{p}=.15)$ erwiesen sich von der zweiten bis zum Ende der vierten Klasse als relativ konstant (vgl. Abbildung 5.14).

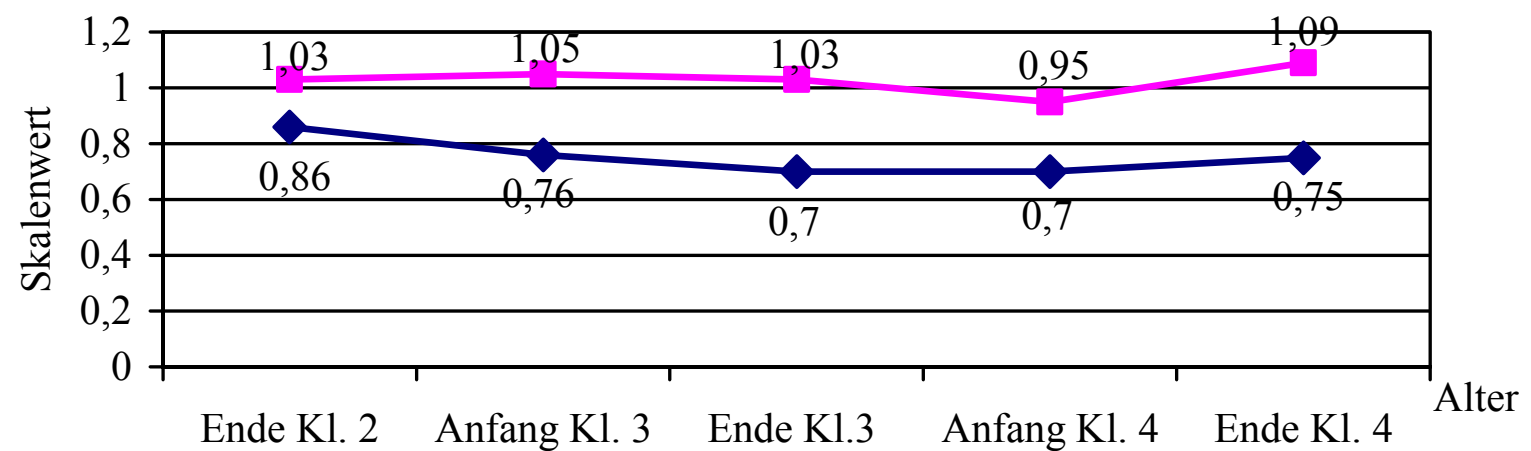

Motivation $\rightarrow$-Selbstkonzept

Abbildung 5.14: Mittlere Motivation und Selbstkonzept in Abhängigkeit vom Alter

Dafür spricht auch die zeitliche Stabilität (mittel- und langfristig) des Selbstkonzepts $(\mathrm{rs} \geq .40, \mathrm{ps}<\alpha)$ und der Motivation ( $\mathrm{rs} \geq .47, \mathrm{ps}<\alpha)$. Eine Differenzierung zwischen verschiedenen Facetten des Selbstkonzepts und der Motivation (z.B. zwischen schulischen und allgemeinen Gedächtnisanforderungen) erscheint nicht notwendig, da sich beide 
Konzepte als relativ homogen erwiesen. Dieses zeigte sich in den bedeutsamen Zusammenhängen zwischen den verschiedenen Komponenten der Variablen (allgemeine Gedächtnisanforderungen und schulische Leistungen) und der hohen zeitlichen Stabilität (mittel- und langfristig), die sowohl beim Selbstkonzept ( $\mathrm{rs} \geq .31$, ps $<\alpha$ ) als auch bei der Motivation ( $\mathrm{rs} \geq .38, \mathrm{ps}<\alpha$ ) gegeben waren. Aus diesem Grund werden im Folgenden nur die Gesamtwerte berichtet.

Darüber hinaus erwies sich ab der dritten Klasse auch der Zusammenhang zwischen der Einschätzung der eigenen Lernfähigkeit (Selbstkonzept) und der Motivation als bedeutsam $(\mathrm{rs} \geq .40, \mathrm{ps}<\alpha)$.

\subsubsection{Determinanten für effektives kategoriales Organisieren}

\section{Die Bedeutung des Metagedächtnisses}

Analysiert werden sollte, in welcher Beziehung das aufgabenspezifische Metagedächtnis und das kategoriale Organisieren zueinander stehen. Hinsichtlich der Richtung des Zusammenhangs zwischen Strategiegebrauch und metakognitivem Wissen gibt es bisher zwei Grundannahmen: Die eine Annahme geht davon aus, dass der Strategiegebrauch zu metakognitiven Erkenntnissen bzw. der Ausbildung eines angemessenen Metagedächtnisses führt (vgl. Flavell, Miller \& Miller, 1993; Schneider \& Pressley, 1997). Dieses soll sich im weiteren Verlauf positiv auf den Strategieerwerb auswirken (vgl. Pressley, Borkowski und Schneider, 1989). Es wird allerdings erwartet, dass die Erlangung eines angemessenen Metagedächtnisses später erfolgt als die Erlangung des Strategenstatus. Daher ist davon auszugehen, dass zuerst das metakognitive Wissen die Entwicklung des strategischen Verhaltens begünstigt. Am Ende der Grundschulzeit (d.h. in der vierten Klasse) kann dann auch erwartet werden, dass das strategische Verhalten die Entwicklung des Metagedächtnisses begünstigt bzw. dass eine wechselseitige Beeinflussung stattfindet.

Die regressionsanalytische Überprüfung dieser Annahme mit dem Metagedächtnis als abhängiger Variable und dem Strategiegebrauch als unabhängigen Variablen erbrachte bis zum Ende der dritten Klasse keine Hinweise auf die Gültigkeit dieser Annahme (vgl. Tabelle 5.14). Unterschiede im Metagedächtnis ließen sich am Ende der zweiten und der dritten Klasse durch das strategische Verhalten zum gleichen Messzeitpunkt erklären. Ab der vierten Klasse änderte sich dieses Bild. Sowohl zu Beginn als auch am Ende der vierten Klasse konnte ein deutlicher Anteil des aufgabenspezifischen Metagedächtnisses durch das Sortierverhalten zum vorherigen Messzeitpunkt erklärt werden. 
Tabelle 5.14: Ergebnisse der Regressionsanalysen für kategoriales Organisieren als Determinanten für das Metagedächtnis

\begin{tabular}{|c|c|c|c|c|c|}
\hline & \multicolumn{2}{|c|}{ Determinanten } & Beta & t-Wert & R-Quadrat \\
\hline Ende Klasse 2 & Zum gleichen MZP & Sortieren & .24 & 2,56 & .058 \\
\hline Anfang Klasse 3 & - & - & - & - & - \\
\hline Ende Klasse 3 & Zum gleichen MZP & Clustern & .31 & 2,98 & .098 \\
\hline Anfang Klasse 4 & Am Ende der 3. Klasse & Sortieren & .43 & 4,26 & .181 \\
\hline Ende Klasse 4 & Zu Beginn der 4. Klasse & Sortieren & .37 & 3,49 & .135 \\
\hline
\end{tabular}

Alternativ wurde bisweilen postuliert, dass ein distales Metagedächtnis eine Voraussetzung für strategisches Verhalten (hier: kategoriales Organisieren) war (vgl. Schlagmüller \& Schneider, 2002; Hasselhorn, 1996). Diese Annahme soll im Folgenden näher untersucht werden.

Die regressionsanalytische Auswertung (vgl. Tabelle 5.15) zeigte, dass zu Beginn der dritten Klasse das Metagedächtnis am Ende der zweiten Klasse sowohl Unterschiede in der Sortier- und Clusterleistung als auch in der Reproduktionsleistung erklärte. Am Ende der dritten Klasse waren erklärungsrelevante Variablen ebenfalls das Metagedächtnis am Ende der zweiten Klasse, aber auch das am Ende der dritten Klasse. Zu Beginn der vierten Klasse erklärte das aktuelle Metagedächtnis die unterschiedliche Verhaltensqualität (d.h. das Ausmaß kategorialer Organisation in beiden Phasen) und Reproduktionsleistung. 
Tabelle 5.15: Ergebnisse der Regressionsanalysen zu der Bedeutung des Metagedächtnisses als Determinante für das kategoriale Organisieren und die Reproduktionsleistung

\begin{tabular}{|c|c|c|c|c|c|}
\hline & & Einfluss des MG & Beta & $\mathrm{t}$-Wert & R-Quadrat \\
\hline \multirow{3}{*}{ Ende K1. 2} & Sortieren & zum gleichen MZP & .25 & 2,31 & .061 \\
\hline & Clustern & - & - & - & - \\
\hline & Reproduktion & zum gleichen MZP & .31 & 2,98 & .096 \\
\hline \multirow{3}{*}{ Anfang K1. 3} & Sortieren & \multirow{3}{*}{ am Ende der 2. Klasse } & .35 & 3,41 & .122 \\
\hline & Clustern & & .36 & 3,49 & .126 \\
\hline & Reproduktion & & .33 & 3,16 & .107 \\
\hline \multirow{6}{*}{ Ende K1. 3} & \multirow{2}{*}{ Sortieren } & am Ende der 2. Klasse & .25 & 2,33 & \multirow[t]{2}{*}{.157} \\
\hline & & zum gleichen MZP & .23 & 2,18 & \\
\hline & \multirow{2}{*}{ Clustern } & am Ende der 2. Klasse & .29 & 2,73 & \multirow{2}{*}{.193} \\
\hline & & zum gleichen MZP & .25 & 2,37 & \\
\hline & \multirow{2}{*}{ Reproduktion } & am Ende der 2. Klasse & .30 & 2,98 & \multirow{2}{*}{.240} \\
\hline & & zum gleichen MZP & .29 & 2,88 & \\
\hline \multirow{3}{*}{ Anfang K1. 4} & Sortieren & \multirow{3}{*}{ zum gleichen MZP } & .40 & 3,96 & .157 \\
\hline & Clustern & & .42 & 4,19 & .173 \\
\hline & Reproduktion & & .42 & 4,29 & .180 \\
\hline \multirow{4}{*}{ Ende K1. 4} & Sortieren & zu Beginn der 4. Klasse & .34 & 3,25 & .117 \\
\hline & \multirow{2}{*}{ Clustern } & am Ende der 2. Klasse & .27 & 2,49 & \multirow{2}{*}{.167} \\
\hline & & zu Beginn der 4. Klasse & .22 & 1,99 & \\
\hline & Reproduktion & zu Beginn der 4. Klasse & .39 & 3,77 & .151 \\
\hline
\end{tabular}




\section{Kombinierter Einfluss des Metagedächtnisses und der Gedächtnisspanne bzw. motivationaler Faktoren auf effektives kategoriales Organisieren}

Um die relative Bedeutung der diskutierten Faktoren für die interindividuellen Leistungsunterschiede bei der effektiven Strategieentwicklung abschätzen zu können, wurden in einem weiteren Auswertungsschritt schrittweise Regressionsanalysen für die einzelnen Messzeitpunkte durchgeführt. Einbezogen wurden das aufgabenspezifische Metagedächtnis und kognitive Faktoren (Zahlenspanne, vorwärts und rückwärts) sowie motivationale Faktoren (Motivation und Selbstkonzept) zum aktuellen Messzeitpunkt.

Die Identifikation der entscheidenden Einflussfaktoren für eine effektive Strategieproduktion erforderte die Suche nach Determinanten, die Unterschiede sowohl im Verhalten in den unterschiedlichen Phasen der Aufgabenbearbeitung als auch Reproduktionsunterschiede erklären können. Als solche Faktoren ließen sich am Ende der dritten Klasse das aufgabenspezifische Metagedächtnis und die Motivation, danach nur das Metagedächtnis identifizieren (vgl. Tabelle 5.16). Darüber hinaus schien am Ende der zweiten Klasse das Metagedächtnis die unterschiedlich effektive Organisationsleistung in der Lernphase, die Gedächtnisspanne (rückwärts) hingegen unterschiedliches Organisieren in der Abrufphase erklären zu können. 
Tabelle 5.16: Ergebnisse der Regressionsanalysen zu Determinanten (Gedächtnisspanne (GS), Metagedächtnis, Motivation, Selbstkonzept) des kategorialen Organisierens (Sortieren, Clustern) und der Reproduktionsleistung

\begin{tabular}{|c|c|c|c|c|c|}
\hline & & Determinanten & Beta & $\mathrm{t}$-Wert & R-Quadrat \\
\hline \multirow{4}{*}{ Ende K1. 2} & Sortieren & Metagedächtnis & .25 & 2,37 & .064 \\
\hline & Clustern & GS (rückwärts) & .22 & 2,0 & .046 \\
\hline & \multirow{2}{*}{ Reproduktion } & GS (rückwärts) & .33 & 3,26 & \multirow{2}{*}{.213} \\
\hline & & Metagedächtnis & .25 & 2,52 & \\
\hline \multirow{4}{*}{ Anfang K1. 3} & Sortieren & - & - & - & - \\
\hline & Clustern & - & - & - & - \\
\hline & \multirow{2}{*}{ Reproduktion } & Metagedächtnis & .23 & 2,23 & \multirow{2}{*}{.166} \\
\hline & & Motivation & .24 & 2,37 & \\
\hline \multirow{6}{*}{ Ende K1. 3} & \multirow{2}{*}{ Sortieren } & Metagedächtnis & .33 & 3,26 & \multirow{2}{*}{.163} \\
\hline & & Motivation & .24 & 2,32 & \\
\hline & \multirow{2}{*}{ Clustern } & Metagedächtnis & .36 & 3,60 & \multirow{2}{*}{.173} \\
\hline & & Motivation & .20 & 2,03 & \\
\hline & \multirow{2}{*}{ Reproduktion } & Metagedächtnis & .41 & 4,24 & \multirow[t]{2}{*}{.234} \\
\hline & & Motivation & .26 & 2,65 & \\
\hline \multirow{3}{*}{ Anfang K1. 4} & Sortieren & \multirow{3}{*}{ Metagedächtnis } & .39 & 3,86 & .152 \\
\hline & Clustern & & .41 & 4,11 & .169 \\
\hline & Reproduktion & & .42 & 4,17 & .173 \\
\hline \multirow{3}{*}{ Ende K1. 4} & Sortieren & \multirow{3}{*}{ Metagedächtnis } & .31 & 2,88 & .094 \\
\hline & Clustern & & .29 & 2,70 & .083 \\
\hline & Reproduktion & & .36 & 3,46 & .130 \\
\hline
\end{tabular}




\section{Die Bedeutung früherer kognitiver Kompetenzen und motivationaler Zustände}

Daran anschließend wurden auch die Determinanten der vorhergehenden Messzeitpunkte in die regressionsanalytische Auswertung miteinbezogen. Die Ergebnisse dieser Analysen sind Tabelle $5.17 \mathrm{zu}$ entnehmen.

Tabelle 5.17: Ergebnisse der Regressionsanalysen zu aktuellen und früheren Determinanten (Gedächtnisspanne (GS), Metagedächtnis, Motivation, Selbstkonzept) des kategorialen Organisierens (Sortieren, Clustern) und der Reproduktionsleistung

\begin{tabular}{|c|c|c|c|c|c|c|}
\hline & & Det & minanten & Beta & t-Wert & R-Quadrat \\
\hline \multirow{6}{*}{$\begin{array}{c}\text { Anfang } \\
\text { K1.3 }\end{array}$} & \multirow{2}{*}{ Sortieren } & \multirow{2}{*}{ Ende K1. 2} & Metagedächtnis & .27 & 2,56 & \multirow{2}{*}{.177} \\
\hline & & & GS (rückwärts) & .27 & 2,54 & \\
\hline & \multirow{2}{*}{ Clustern } & \multirow[t]{2}{*}{ Ende K1. 2} & Metagedächtnis & .29 & 2,78 & \multirow{2}{*}{.165} \\
\hline & & & GS (vorwärts) & .25 & 2,38 & \\
\hline & \multirow{2}{*}{ Reproduktion } & \multirow{2}{*}{ Ende K1. 2} & GS (rückwärts) & .37 & 3,68 & \multirow{2}{*}{.217} \\
\hline & & & Metagedächtnis & .27 & 2,75 & \\
\hline \multirow{5}{*}{$\begin{array}{l}\text { Ende } \\
\text { K1. } 3\end{array}$} & Sortieren & aktuell & Metagedächtnis & .37 & 3,53 & .135 \\
\hline & \multirow{2}{*}{ Clustern } & aktuell & Metagedächtnis & .33 & 3,23 & \multirow{2}{*}{.206} \\
\hline & & Ende K1. 2 & GS (rückwärts) & .24 & 2,37 & \\
\hline & \multirow{2}{*}{ Reproduktion } & aktuell & Metagedächtnis & .28 & 2,86 & \multirow{2}{*}{.331} \\
\hline & & Ende Kl. 2 & GS (rückwärts) & .30 & 3,20 & \\
\hline \multirow{5}{*}{$\begin{array}{c}\text { Anfang } \\
\text { K1. } 4\end{array}$} & \multirow{2}{*}{ Sortieren } & aktuell & Metagedächtnis & .36 & 3,46 & \multirow{2}{*}{.228} \\
\hline & & Anfang K1. 3 & Metagedächtnis & .23 & 2,19 & \\
\hline & \multirow{2}{*}{ Clustern } & aktuell & Metagedächtnis & .38 & 3,63 & \multirow{2}{*}{.243} \\
\hline & & Ende Kl. 2 & GS (rückwärts) & .23 & 2,19 & \\
\hline & Reproduktion & aktuell & Metagedächtnis & .43 & 4,26 & .187 \\
\hline \multirow{7}{*}{$\begin{array}{l}\text { Ende } \\
\text { Kl. } 4\end{array}$} & \multirow{2}{*}{ Sortieren } & Ende Kl. 2 & GS (rückwärts) & .22 & 2,00 & \multirow{2}{*}{.178} \\
\hline & & Anfang K1. 4 & Metagedächtnis & .29 & 2,64 & \\
\hline & \multirow{4}{*}{ Clustern } & $\Gamma_{1} V_{1}$ & Metagedächtnis & .28 & 2,65 & \multirow{4}{*}{.274} \\
\hline & & Ende Kl. 2 & GS (rückwärts) & .24 & 2,18 & \\
\hline & & Anfang K1. 3 & GS (vorwärts) & -.33 & 2,73 & \\
\hline & & Anfang K1. 4 & GS (vorwärts) & .35 & 2,94 & \\
\hline & Reproduktion & Anfang K1. 4 & Metagedächtnis & .39 & 3,68 & .153 \\
\hline
\end{tabular}


Es zeigte sich, dass Unterschiede in der Effektivität des strategischen Verhaltens bis zum Ende der dritten Klasse ausschließlich mit kognitiven Determinanten zum gleichen Messzeitpunkt und vom Beginn der Untersuchung (Ende Klasse 2) erklären ließen. Erst in der vierten Klasse spielten auch kognitive Einflussfaktoren aus anderen Altersabschnitten eine Rolle. So klärte das metakognitive Wissen $\mathrm{zu}$ Beginn der dritten Klasse auch Sortierunterschiede zu Beginn der vierten Klasse auf. Zu Beginn der vierten Klasse ließ sich das effektive Sortieren durch das Metagedächtnis zu Beginn der vierten Klasse erklären. Prädiktoren für das kategoriale Organisieren in der Abrufphase waren dagegen neben dem Metagedächtnis und der Gedächtnisspanne (rückwärts) am Ende der zweiten Klasse die Gedächtnisspanne (vorwärts) zu Beginn der dritten und vierten Klasse.

Sowohl am Ende der dritten Klasse als auch zu Beginn der vierten Klasse stellte das Metagedächtnis zum gleichen Messzeitpunkt eine wichtige Einflussgröße für die effektive Strategieproduktion dar, da es sowohl das kategoriale Organisieren in Lern- und Abrufphase als auch die Reproduktionsleistung determinierte. Am Ende der zweiten Klasse erklärte das Metagedächtnis einen bedeutsamen Varianzanteil der Unterschiede in der Sortier- und Erinnerungsleistung. $\mathrm{Zu}$ Beginn der dritten Klasse trug das metakognitive Wissen zum vorausgehenden Messzeitpunkt (Ende Klasse 2) dazu bei, ein unterschiedliches Ausmaß kategorialer Organisation in Lern- und Abrufphase zu erklären. Für die Erklärung der Reproduktionsunterschiede $\mathrm{zu}$ Beginn der dritten Klasse erwies sich das aktuelle metakognitive Wissen als geeigneter.

Unterschiede in der Effektivität der Sortierleistung am Ende der vierten Klasse ließen sich durch das aufgabenspezifische Metagedächtnis, das die Kinder ein halbes Jahr zuvor gezeigt hatten (Anfang Klasse 4) erklären. Zur Erklärung des Ausmaßes kategorialer Organisation beim Abruf lieferte das metakognitive Wissen am Ende der zweiten Klasse einen höheren Beitrag.

Eine weitere wichtige Determinante schien die Gedächtnisspanne (rückwärts) am Ende der zweiten Klasse zu sein. Am Ende der zweiten und dritten Klasse erklärte sie Unterschiede in der Effektivität des Clustern in der Abrufphase, zu Beginn der dritten Klasse die Effektivität des Sortierens. Am Ende der vierten Klasse erklärte die frühe Gedächtnisspanne (rückwärts) unterschiedliches Kategorisieren in Lern- und Abrufphase. Mit Ausnahme des Beginns der dritten Klasse, in der sich die Gedächtnisspanne (vorwärts) am Ende der zweiten Klasse als erklärungsstärker herausgestellt hatte, half die Gedächtnisspanne (rückwärts) am Ende der zweiten Klasse zu jedem Messzeitpunkt die unterschiedlichen 
Clusterleistungen zu erklären. Der Einfluss auf die Reproduktionsleistung beschränkte sich hingegen auf die Zeit bis zum Ende der dritten Klasse

\section{Determinanten für eine gute Gedächtnisleistung:}

Folgenden Fragestellungen hinsichtlich möglicher Einflussfaktoren auf die Güte der Gedächtnisleistung soll nachgegangen werden:

- $\quad$ Ist die Gedächtnisleistung primär durch das strategische Verhalten vorhersagbar?

- Welche anderen kognitiven und motivationalen Faktoren (neben dem

Strategiegebrauch) können Unterschiede in der Reproduktionsleistung erklären?

Die diskutierten Determinanten sollten nicht nur einen Einfluss auf den effektiven Strategiegebrauch, sondern darüber hinaus auf die Qualität der gesamten Gedächtnisleistung haben. Diese über die reine Strategieproduktion hinausgehende Bedeutung soll im Folgenden näher analysiert werden.

Um die relative Bedeutung der diskutierten Faktoren für die interindividuellen Unterschiede bei der Gedächtnisleistung abschätzen zu können, wurden in einem weiteren Auswertungsschritt schrittweise Regressionsanalysen für die einzelnen Messzeitpunkte durchgeführt. Einbezogen wurden das Ausmaß kategorialer Organisation in Lern- und Abrufphase, das Metagedächtnis und kognitive Faktoren (Gedächtnisspanne vorwärts und rückwärts) sowie motivationale Faktoren (Motivation und Selbstkonzept) zum aktuellen Messzeitpunkt als auch zu vorhergehenden Messzeitpunkten. Die Ergebnisse dieser Analysen sind Tabelle $5.18 \mathrm{zu}$ entnehmen. 
Tabelle 5.18: Ergebnisse der Regressionsanalysen zu Determinanten der Gedächtnisleistung (Gedächtnisspanne (GS), Metagedächtnis, Motivation, Selbstkonzept)

\begin{tabular}{|c|c|c|c|c|}
\hline & Determinanten & Beta & t-Wert & R-Quadrat \\
\hline \multirow{4}{*}{ Ende Klasse 2 } & Sortieren & .48 & 4,70 & \multirow{2}{*}{.351} \\
\cline { 2 - 4 } & Clustern & -.25 & 2,41 & \\
\cline { 2 - 4 } & GS (rückwärts) & .37 & 4,02 & \\
\hline \multirow{4}{*}{ Anfang Klasse 3 } & Sortieren & .34 & 2,72 & \multirow{2}{*}{.504} \\
\cline { 2 - 4 } & Clustern & .33 & 2,68 & \\
\cline { 2 - 4 } & Metagedächtnis & .19 & 2,34 & \\
\cline { 2 - 4 } & Motivation & .18 & 2,27 & \\
\hline \multirow{5}{*}{ Ende Klasse 3 } & Sortieren & .61 & 7,59 & \multirow{2}{*}{.543} \\
\cline { 2 - 4 } & Metagedächtnis & .21 & 2,63 & \multirow{2}{*}{.491} \\
\cline { 2 - 4 } & Selbstkonzept & .19 & 2,47 & \\
\hline \multirow{2}{*}{ Anfang Klasse 4 } & Sortieren & .43 & 3,07 & \multirow{2}{*}{.491} \\
\hline
\end{tabular}

Während das kategoriale Organisieren in der Lernphase (Sortieren) zu jeder Zeit die entscheidende Determinante zur Erklärung der Leistungsunterschiede darstellte, schien der Beitrag des kategoriale Organisieren in der Abrufphase (Clustern) auf das Ende der zweiten, den Beginn der dritten und den Anfang der vierten Klasse beschränkt zu sein. Die Gedächtnisspanne (rückwärts) erklärte noch am Ende der zweiten Klasse einen bedeutsamen Teil der unterschiedlichen Erinnerungsleistung. In der dritten Klasse leisteten das Metagedächtnis sowie ein motivationaler Faktor einen wesentlichen Beitrag. Zu Beginn der dritten Klasse handelte es sich dabei um die Motivation, am Ende der dritten Klasse um das Selbstkonzept der Kinder.

\section{Besonderheiten unter vertrauten Anforderungen (bei hoher kategorialer} Typizität der Items in der Mitte der Grundschulzeit und erhöhter Itemanzahl am

\section{Ende der Grundschulzeit)}

Am Ende der zweiten Klasse klärten die Sortierleistung und die Gedächtnisspanne rückwärts 62\% der Reproduktionsunterschiede in der zweiten Liste auf. Zu Beginn der dritten Klasse ließ sich die Höhe der Gedächtnisleistung ausreichend mit Hilfe des kategorialen Organisierens in Lern- und Abrufphase vorhersagen (58,4\%). 
Am Ende der dritten und zu Beginn der vierten Klasse erklärte neben dem kategorialen Organisieren in der Lernphase (Sortieren) auch die Gedächtnisspanne (rückwärts) einen bedeutsamen Anteil der Gedächtnisleistung (vgl. Tab. 5.19). Am Ende der dritten Klasse lieferte darüber hinaus auch das Selbstkonzept einen bedeutsamen Beitrag. Am Ende der vierten Klasse stellte das Metagedächtnis eine wichtige Einflussgröße dar.

Tabelle 5.19: Ergebnisse der Regressionsanalysen zu Determinanten (Metagedächtnis, Gedächtnisspanne (GS), Motivation, Selbstkonzept) für die Gedächtnisleistung

\begin{tabular}{|c|c|c|c|c|}
\hline \multirow{2}{*}{ Ende Klasse 3 } & Determinanten & Beta & t-Wert & R-Quadrat \\
\cline { 2 - 4 } & Sortieren & .53 & 6,30 & \multirow{2}{*}{.442} \\
\cline { 2 - 4 } & GS (rückwärts) & .23 & 2,67 & \multirow{2}{*}{.446} \\
\cline { 2 - 4 } Anfang Klasse 4 & Selbstkonzept & .18 & 2,11 & \\
\cline { 2 - 4 } & Sortieren & .63 & 7,60 & \multirow{2}{*}{.490} \\
\hline \multirow{2}{*}{ Ende Klasse 4 } & GS (rückwärts) & .22 & 2,69 & \\
\cline { 2 - 4 } & Sortieren & .60 & 7,18 & \multirow{2}{*}{2,57} \\
\hline
\end{tabular}

\subsubsection{Determinanten für einen effektiven bewussten Einsatz der kategorialen Organisationsstrategie}

Obwohl bereits geklärt wurde, dass das metakognitive Wissen über Strategien ein wesentlicher Faktor für die Anwendung der Strategie ist, bleibt offen, ob ein angemessenes Metagedächtnis eine notwendige oder hinreichende Bedingung für die Produktion der kategorialen Organisationsstrategie darstellt. Zur Überprüfung diese Annahme wurden Kinder entsprechend der Angemessenheit ihres Strategiewissens (Metagedächtnis) klassifiziert. Ein angemessenes aufgabenspezifisches Metagedächtnis wurde den Kindern zugesprochen, die in dem Metagedächtnisfragebogen einen Itemblock (d.h. entweder aus dem Messinstrument von Schlagmüller et al. (2001) oder von Hasselhorn (1994)) vollständig richtig beantworteten.

Der Anteil der Kinder, die die Listenitems oder Wortlistenitems perfekt beantworteten, steigerte sich vom Beginn der dritten Klasse (17,4\%) zum Ende auf 31,4\% (vgl. Abbildung 5.15). Bis zum Beginn der vierten Klasse änderte sich dieser Anteil auch nicht wesentlich 
(38,4\%), am Ende der vierten Klasse konnte allerdings eine deutliche Steigerung beobachtet werden. $\mathrm{Zu}$ diesem Zeitpunkt verfügten $72 \%$ der Kinder über ein angemessenes aufgabenspezifisches Metagedächtnis.

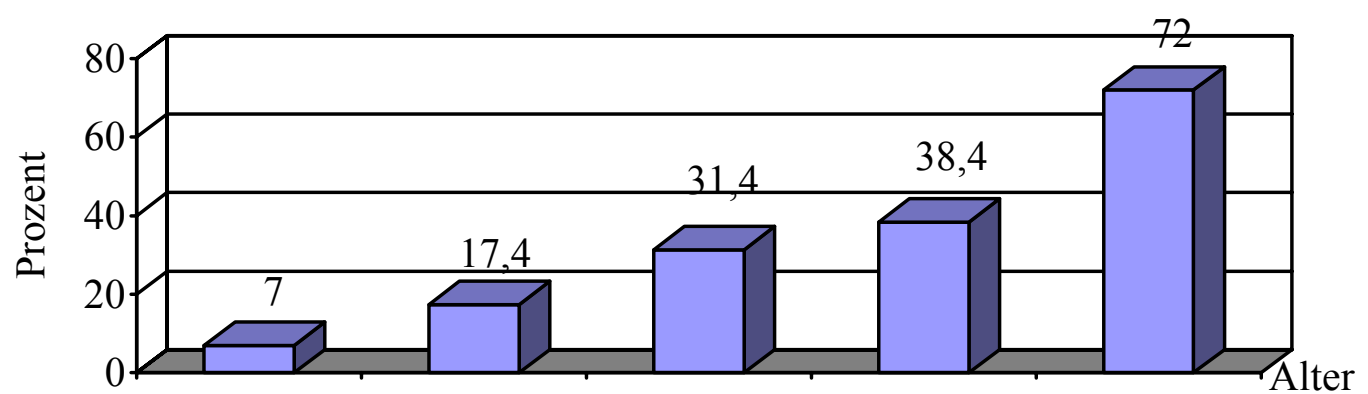

Ende Kl. 2 Anfang Kl. 3 Ende Kl. 3 Anfang Kl. 4 Ende Kl. 4

$\square$ Deklaratives MG

Abbildung 5.15: Häufigkeitsverteilung der Kinder mit angemessenem Metagedächtnis in Abhängigkeit von den verschiedenen Altersstufen

In Bezug auf den Zusammenhang zwischen der Angemessenheit des Metagedächtnisses und dem Strategenstatus wurde erwartet, dass die kategoriale Organisationsstrategie auch ohne angemessenes Metagedächtnis gezeigt wird.

Vergleicht man, wie viele Strategen über ein angemessenes Metagedächtnis verfügen, so zeigt sich, dass es nur zu Beginn der dritten Klasse $(\Phi=.381)$ und zu Beginn der vierten Klasse $(\Phi=.274)$ einen bedeutsamen Zusammenhang gab. Am Ende der zweiten Klasse verfügte noch kein Stratege gleichzeitig über ein angemessenes Metagedächtnis. Die Kinder mit metakognitivem Wissen wendeten umgekehrt in der kategorialen Organisationsaufgabe die erforderliche Strategie nicht an. Zu Beginn der dritten Klasse änderte sich das Bild: Zwei Drittel der Kinder mit angemessenem metakognitiven Wissen verhielten sich bei der SortRecall-Aufgabe strategisch. Im Laufe der Zeit nahm der Anteil an Strategen ebenso wie der Anteil an Kindern mit guten metakognitiven Kenntnissen weiter zu. Aber erst zu Beginn der vierten Klasse verfügte die Hälfte der Strategen über ein angemessenes Metagedächtnis. Dieser Anteil stieg bis zum Ende der Grundschulzeit noch weiter (vgl. Tabelle 5.20). Es gab aber immer noch 10 Strategen ohne entsprechendes metakognitives Wissen und 24 Kinder, die trotz ihres Wissens in der Sort-Recall-Aufgabe die kategoriale Organisationsstrategie nicht vollständig einsetzten. 
Tabelle 5.20: Bivariate Häufigkeitsverteilungen und empirische Phi-Werte für die Zusammenhangsüberprüfung zwischen dem Metagedächtnis und dem Strategenstatus

\begin{tabular}{|c|c|c|c|c|}
\hline \multirow{2}{*}{\multicolumn{2}{|c|}{ Strategenstatus }} & \multicolumn{2}{|c|}{ Metagedächtnis } & \\
\hline & & Nicht angemessen & angemessen & \\
\hline \multirow{2}{*}{ Ende K1. 2} & Nicht-Stratege & 71 & 6 & \multirow{2}{*}{$\Phi=-.094$ (n.s.) } \\
\hline & Stratege & 9 & 0 & \\
\hline \multirow[t]{2}{*}{ Anfang K1. 3} & Nicht-Stratege & 56 & 5 & \multirow[t]{2}{*}{$\Phi=.381$} \\
\hline & Stratege & 15 & 10 & \\
\hline \multirow{2}{*}{ Ende K1. 3} & Nicht-Stratege & 37 & 11 & \multirow{2}{*}{$\Phi=.205$ (n.s.) } \\
\hline & Stratege & 22 & 16 & \\
\hline \multirow[t]{2}{*}{ Anfang K1. 4} & Nicht-Stratege & 31 & 10 & \multirow{2}{*}{$\Phi=.274$} \\
\hline & Stratege & 22 & 23 & \\
\hline \multirow{2}{*}{ Ende K1. 4} & Nicht-Stratege & 13 & 24 & \multirow[t]{2}{*}{$\Phi=.143$ (n.s.) } \\
\hline & Stratege & 10 & 35 & \\
\hline
\end{tabular}

\section{Zusammenhang zwischen der Angemessenheit des Metagedächtnisses und der Effektivität der Strategieproduktion}

Darüber hinaus stellte sich die Frage nach einem Zusammenhang zwischen der Angemessenheit des Metagedächtnisses und der Effektivität der Strategieproduktion. Ein angemessenes Metagedächtnis sollte in Zusammenhang mit der Effektivität des Strategiegebrauchs stehen.

Am Ende der zweiten Klasse verfügte keins der strategischen Kinder über ein angemessenes aufgabenspezifisches Metagedächtnis, d.h. keinem Kind gelang es, eine Itemgruppe im Fragebogen perfekt zu beantworten. Wie Tabelle $5.21 \mathrm{zu}$ entnehmen ist, verfügte ab Beginn der vierten Klasse die Mehrheit der effektiven Strategen über ein angemessenes metakognitives Wissen, ab Ende der vierten Klasse auch die Mehrheit der ineffektiven Strategen. 
Tabelle 5.21: Häufigkeitsverteilung der Strategen mit angemessenem Metagedächtnis in Abhängigkeit von der Effektivität der Strategieproduktion (Anteil an Strategentyp in Klammern)

\begin{tabular}{|c|c|c|c|c|}
\hline \multirow{2}{*}{} & \multicolumn{4}{|c|}{ Angemessenes Metagedächtnis } \\
\cline { 2 - 5 } & $\begin{array}{c}\text { Anfang K1. } 3 \\
\mathrm{~N}=15\end{array}$ & $\begin{array}{c}\text { Ende K1. 3 } \\
\mathrm{N}=27\end{array}$ & $\begin{array}{c}\text { Anfang K1. } 4 \\
\mathrm{~N}=33\end{array}$ & $\begin{array}{c}\text { Ende K1. } 4 \\
\mathrm{~N}=59\end{array}$ \\
\hline Ineffektive Strategen & $\mathrm{N}=2(28,6 \%)$ & $\mathrm{N}=3(30 \%)$ & $\mathrm{N}=6(46,2 \%)$ & $\mathrm{N}=9(56,3 \%)$ \\
\hline Effektive Strategen & $\mathrm{N}=8(44,4 \%)$ & $\mathrm{N}=13(46,4 \%)$ & $\mathrm{N}=17(53,1 \%)$ & $\mathrm{N}=26(89,7 \%)$ \\
\hline
\end{tabular}

Der Anteil metakognitiv kompetenter Kinder war unter den effektiven Strategen stets etwas höher als unter den nutzungsdefizitären Strategen, wobei besonders am Ende der vierten Klasse der Unterschied deutlich wurde (89,7\% vs. 56,3\%). Am Ende der vierten Klasse war dieser Unterschied bedeutsam (vgl. Tabelle 5.22).

Tabelle 5.22: Bivariate Häufigkeitsverteilungen und empirische Phi-Werte für die Zusammenhangsüberprüfung zwischen Angemessenheit des Metagedächtnisses und der Effektivität der Strategieproduktion bei den Strategen am Ende der vierten Klasse

\begin{tabular}{|c|c|c|c|c|}
\hline \multicolumn{2}{|c|}{$\Phi=.385$} & \multicolumn{3}{c|}{ Metagedächtnis } \\
\cline { 3 - 5 } \multicolumn{2}{|c|}{} & Nicht angemessen & angemessen & \\
\hline \multirow{2}{*}{$\begin{array}{c}\text { Strategen- } \\
\text { status }\end{array}$} & ineffektiver Stratege & 7 & 9 & 16 \\
\cline { 2 - 5 } & Effektiver Stratege & 3 & 26 & 29 \\
\hline & 10 & 35 & 45 \\
\hline
\end{tabular}

\section{Hinreichende Bedingungen für die effektive Strategieproduktion}

Neben der Bedeutung für die Anwendung der Strategie galt es zu klären, ob ein angemessenes Metagedächtnis und eine hohe Gedächtnisspanne zusammen eine hinreichende Bedingung für die effektive Strategieproduktion darstellen. Im Folgenden soll der Zusammenhang zwischen der Nützlichkeit bei der Strategieproduktion und der kombinierten Wirkung von metakognitiven und kognitiven Faktoren näher betrachtet werden. Wenn das Metagedächtnis alleine keine notwendige oder hinreichende Bedingung für die effektive Strategieproduktion darstellt, so muss geklärt werden, ob sich dieses durch Kombination mit anderen Faktoren ändern lässt. Zur Überprüfung dieses Zusammenhangs wurde die 
Häufigkeitsverteilung der Strategen in Abhängigkeit von ihrer Einordnung aufgrund ihres aufgabenspezifischen Metagedächtnisses (Kriterium für angemessenes MG: mindestens ein Itemkomplex musste vollkommen richtig beantwortet werden) und ihrer Gedächtnisspanne (Zahlenspanne rückwärts) betrachtet.

Unter den effektiven Strategen waren die kognitiven und metakognitiven Kompetenzen sehr unterschiedlich verteilt, d.h. es waren keine notwendigen Bedingungskonstellationen identifizierbar. Für einen effektiven Strategieeinsatz am Ende der dritten Klasse konnte allerdings eine hinreichende Bedingungskonstellation gefunden werden. Alle Kinder, die über eine überdurchschnittliche Gedächtnisspanne (rückwärts) und ein angemessenes Metagedächtnis verfügten, erwiesen sich als effektive Strategen (vgl. Tabelle $5.23)$.

Tabelle 5.23: Bivariate Häufigkeitsverteilungen der Strategen mit überdurchschnittlicher Gedächtnisspanne (rückwärts), in Abhängigkeit von der Angemessenheit des Metagedächtnisses (MG) und der Effektivität der Strategieproduktion (Ende Kl. 3)

\begin{tabular}{|c|c|c|c|c|}
\hline \multirow{2}{*}{$\Phi=.522(\mathrm{p}=.051)$} & \multicolumn{2}{c|}{ Strategenstatus } & \\
\cline { 3 - 5 } & $\begin{array}{c}\text { Effektive } \\
\text { Strategen }\end{array}$ & $\begin{array}{c}\text { Ineffektive } \\
\text { Strategen }\end{array}$ & \\
\hline $\begin{array}{c}\text { überdurchschnittliche } \\
\text { Gedächtnisspanne } \\
\text { (rückwärts) }\end{array}$ & unangemessenes MG & 4 & 3 & 7 \\
\cline { 2 - 5 } & angemessenes MG & 7 & 0 & 7 \\
\hline & & 11 & 3 & 14 \\
\hline
\end{tabular}

\section{Analysen auf Individualebene}

Auf der Suche nach Determinanten, die auf Individualniveau für den effektiven Gebrauch der Strategie in bestimmten Entwicklungsphasen relevant sind, soll folgenden Fragen nachgegangen werden: Worin unterscheiden sich effektive Strategen und Kinder mit einem Nutzungsdefizit? Gibt es charakteristische Besonderheiten in Abhängigkeit von dem betrachteten Altersspektrum?

Zur Beantwortung dieser Fragen wurden die kognitiven Leistungsmerkmale und die motivationale Befindlichkeit von effektiven und ineffektiven Strategen miteinander verglichen. Die Mittelwertsvergleiche zeigten, dass effektive Strategen zu Beginn und am 
Ende der vierten Klasse sich gegenüber ineffektiven Strategen durch bessere metakognitive Kompetenzen ein halbes Jahr vor ihrem Strategiegebrauch $\left(t_{\text {Strategen Anfang Kl.4 }}(43)=3,31\right.$; $t_{\text {Strategen }}$ Ende Kl.4 $(43)=2,85, \mathrm{ps}<\alpha)$ und bei ihrem Strategiegebrauch $\left(\mathrm{t}_{\text {Anfang Kl. } 4}(43)=2,58\right.$; $\mathrm{t}_{\text {Ende }} \mathrm{Kl} .4$ $(43)=2,89$, ps $<\alpha$ ) auszeichneten. Effektive Strategen zu Beginn der vierten Klasse verfügten darüber hinaus bereits am Ende der zweiten Klasse über bessere metakognitive Kompetenzen $\left(\mathrm{t}_{\text {Strategen Anfang K1.4 }}(29,7)=2,43, \mathrm{p}<\alpha\right)$. Die diesen Analysen zugrunde liegenden Mittelwerte und Standardabweichungen des ermittelten aufgabenspezifischen Metagedächtnisses zu den verschiedenen Messzeitpunkten sind Abbildung 5.16 zu entnehmen.

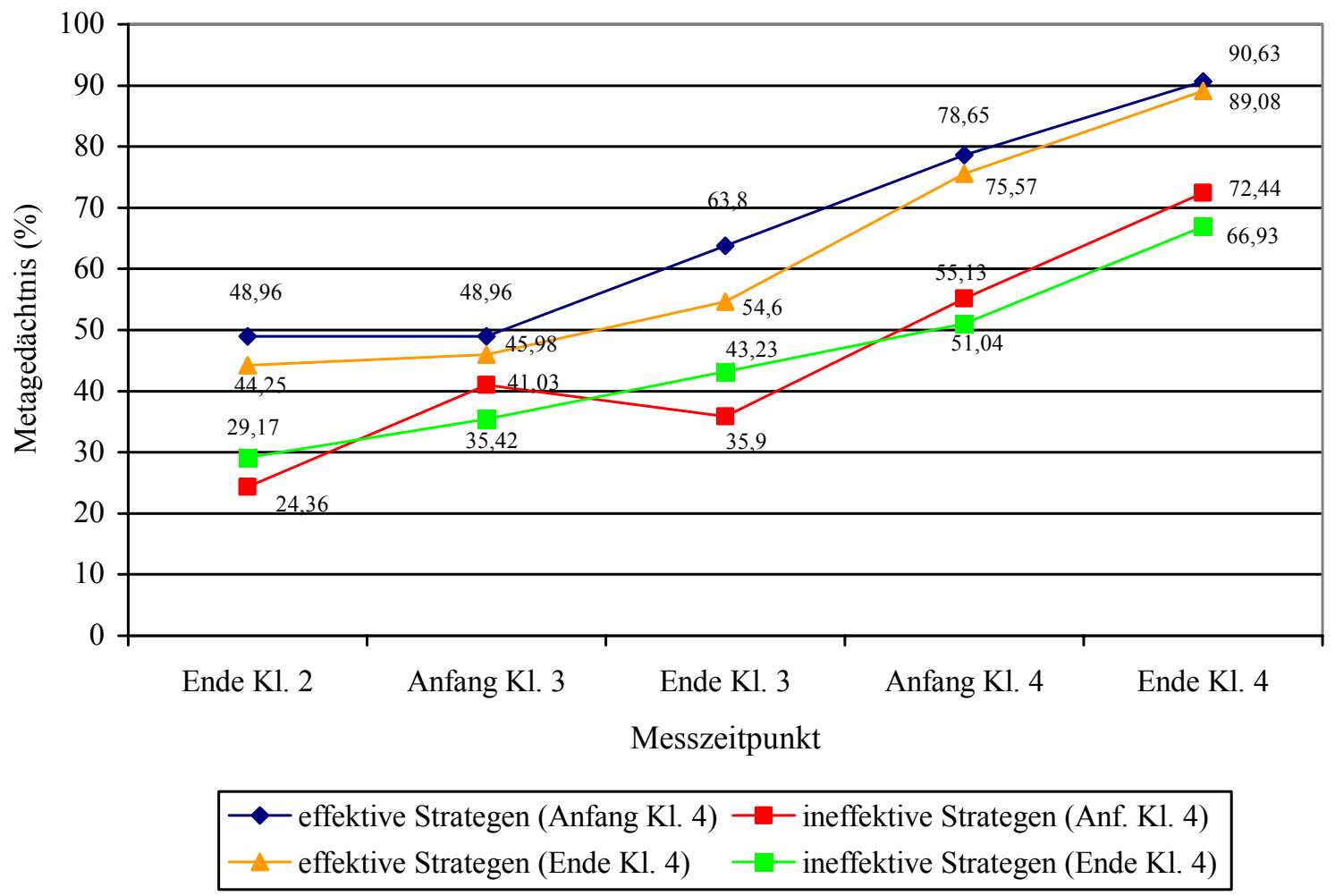

Abbildung 5.16: Aufgabenspezifisches Metagedächtnis (prozentualer Anteil richtig beantworteter Items) von Strategen zu verschiedenen Messzeitpunkten in Abhängigkeit von der Effektivität der Strategieproduktion in der vierten Klasse

Außerdem waren Unterschiede in der kognitiven Leistungsfähigkeit zu verzeichnen, was sich bei den effektiven Strategen zu Beginn der vierten Klasse in Form einer höheren Gedächtnisspanne vorwärts $(M=6 ; S D=1,27)$ gegenüber ineffektiven Strategen $(M=5 ; S D$ : $0,76)$ äußerte $(\mathrm{t}(43)=2,64, \mathrm{p}<\alpha)$. 


\section{Weitere Merkmale zu Beginn der Untersuchung (am Ende der 2. Klasse)}

Wie Tabelle 5.24 zeigt hatten effektive Strategen am Ende der dritten Klasse und zu Beginn der vierten Klasse im Vergleich zu gleichaltrigen uneffektiven Strategen zu Beginn der Untersuchung, d.h. am Ende der zweiten Klasse, ein besseres Selbstkonzept ( $\mathrm{t}$ Ende K1. 2 $(35)=2,29$ bzw. $t$ Ende Kl. $2(42)=2,38, \mathrm{ps}<\alpha)$.

Tabelle 5.24: Selbstkonzept am Ende der zweiten Klasse von Strategen am Ende der 3. Klasse und Anfang der 4. Klasse

\begin{tabular}{|c|c|c|}
\hline \multicolumn{2}{|c|}{} & Selbstkonzept (Ende 2. Klasse) \\
\hline \multirow{2}{*}{ Ende Klasse 3 } & Ineffektive Strategen $(\mathrm{N}=9)$ & $0,7(0,46)$ \\
\cline { 2 - 3 } & Effektive Strategen $(\mathrm{N}=28)$ & $1,17(0,55)$ \\
\hline \multirow{2}{*}{ Anfang Klasse 4 } & Ineffektive Strategen $(\mathrm{N}=13)$ & $0,74(0,57)$ \\
\cline { 2 - 3 } & Effektive Strategen $(\mathrm{N}=32)$ & $1,21(0,55)$ \\
\hline
\end{tabular}

Strategen am Ende der vierten Klasse wiesen am Ende der zweiten Klasse eine höhere Gedächtnisspanne $\left(\mathrm{t}_{\mathrm{ZNvor}}(43)=3,21 ; \mathrm{t}_{\mathrm{ZNrück}}(43)=2,57, \mathrm{ps}<\alpha\right)$ und am Ende der dritten Klasse eine höhere Gedächtnisspanne rückwärts auf $(\mathrm{t}(43)=2,51, \quad \mathrm{p}<\alpha)$. Die dazugehörigen Mittelwerte und Standardabweichungen sind Tabelle $5.25 \mathrm{zu}$ entnehmen.

Tabelle 5.25: Gedächtnisspanne (GS) von am Ende der vierten Klasse identifizierten Strategen

\begin{tabular}{|c|c|c|c|}
\hline \multirow{2}{*}{} & \multicolumn{2}{|c|}{ Ende Klasse 2 } & Ende Klasse 3 \\
\cline { 2 - 4 } & GS vorwärts & GS rückwärts & GS rückwärts \\
\hline Ineffektive Strategen $(\mathrm{N}=16)$ & $4,41(0,74)$ & $3,56(0,51)$ & $3,72(0,77)$ \\
\hline Effektive Strategen $(\mathrm{N}=29)$ & $5,14(0,73)$ & $4,05(0,66)$ & $4,31(0,75)$ \\
\hline
\end{tabular}

\section{Determinanten für eine effektive erste Produktion der Organisationsstrategie}

Es soll den Fragen nachgegangen werden,

- $\quad$ ob Strategieentdecker über bessere metakognitive Fähigkeiten als Nichtstrategen verfügen und

- ob Strategieverlierer eine geringere Motivation und/oder ein niedrigeres Selbstkonzept als konsistente Strategen zeigen.

Da strategisches Verhalten früher beobachtbar war als das Vorhandensein eines angemessenen aufgabenspezifischen Metagedächtnisses, sollte überprüft werden, wie viele 
Kinder bereits bei der ersten Strategieproduktion über ein angemessenes Metagedächtnis verfügten.

Wie die Ergebnisse zeigen, gab es bis zum Beginn der vierten Klasse unter den Kindern mit angemessenem Metagedächtnis nur einen geringen Anteil an Kindern, die zum ersten Mal die Strategie einsetzten (vgl. Tabelle 5.26). Es waren auch keine systematischen Unterschiede hinsichtlich der Verteilung zwischen effektiven und ineffektiven Strategieentdeckern erkennbar. Allerdings ist dabei $\mathrm{zu}$ bedenken, dass die Anzahl der erstmaligen Strategen insgesamt relativ gering ist.

Tabelle 5.26: Anteil der Kinder mit angemessenem aufgabenspezifischem Metagedächtnis zum Zeitpunkt der erstmaligen Strategieproduktion in Abhängigkeit von der Strategieeffektivität

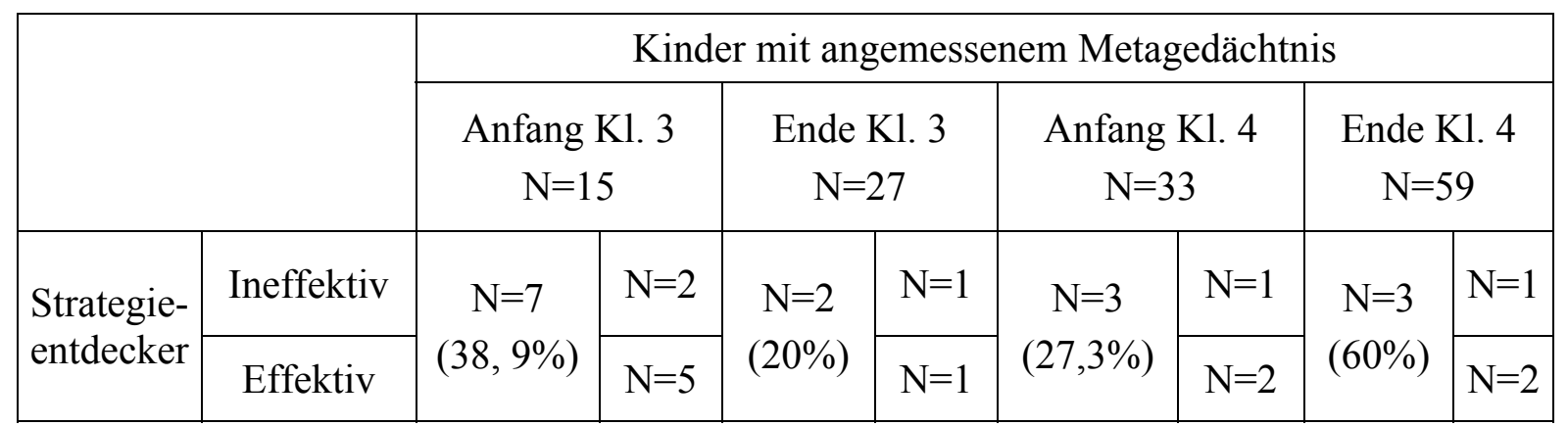

\section{Determinanten für das Entdecken der Organisationsstrategie}

Zur Beantwortung der Frage, ob es Determinanten gibt, die für das Entdecken der Strategie in bestimmten Entwicklungsphasen relevant sind, soll im Folgenden versucht werden, spezifische Unterscheidungsmerkmale zwischen Nicht-Strategen und Strategieentdeckern zu identifizieren.

Es zeigte sich, dass Strategieentdecker zu Beginn der dritten Klasse sich von NichtStrategen hinsichtlich ihres metakognitiven Wissens unterschieden (vgl. Tabelle 5.27). Kinder, die zu Beginn der dritten Klasse die kategoriale Organisationsstrategie entdeckten, verfügten seit der zweiten Klasse über ein besseres Metagedächtnis als Nicht-Strategen $\left(t_{\text {Ende }}\right.$ $\left.\mathrm{K} 1.2(75)=4,83 ; \mathrm{t}_{\text {Anfang K1.3 }}(75)=2,94, \mathrm{ps}<\alpha\right)$. 
Tabelle 5.27: Aufgabenspezifisches Metagedächtnis (prozentualer Anteil richtig beantworteter Items, Standardabweichung in Klammern) in Abhängigkeit vom Strategentypus

\begin{tabular}{|c|c|c|}
\hline \multirow{2}{*}{ Metagedächtnis } & \multicolumn{2}{|c|}{ Ende K1. 2 - Anfang K1. 3 } \\
\cline { 2 - 3 } & Entdecker $(\mathrm{N}=18)$ & Nichtstrategen $(\mathrm{N}=59)$ \\
\hline Ende K1. 2 & $61,11(33,3)$ & $24,29(26,68)$ \\
\hline Anfang K1. 3 & $57,41(40,91)$ & $30,51(31,74)$ \\
\hline Ende K1. 3 & $61,11(30,38)$ & $44,21(24,67)$ \\
\hline Anfang K1. 4 & $76,39(31,47)$ & $52,89(33,49)$ \\
\hline Ende K1. 4 & $89,35(16,43)$ & $69,94(31,5)$ \\
\hline
\end{tabular}

Strategieentdecker zu Beginn der dritten Klasse verfügten darüber hinaus über einen besseren Wortschatz als Nichtstrategen $(\mathrm{t}(75)=4,83, \mathrm{p}<\alpha)$.

\section{Determinanten für das Aufgeben der Organisationsstrategie}

Zur Beantwortung der Frage, ob es Determinanten gibt, die für das Aufgeben der Strategie in bestimmten Entwicklungsphasen relevant sind, soll im Folgenden versucht werden, spezifische Unterscheidungsmerkmale zwischen Strategen und Strategieverlierern zu identifizieren.

Da in der dritten Klasse weniger als 5\% der Kinder die Strategie aufgaben, beschränkt sich die differentielle Analyse auf die Strategieverlierer in der vierten Klasse $\left(\mathrm{N}_{(\text {Anfang Kl. 4) }}=5\right.$; $\mathrm{N}_{\text {(Ende Kl. 4) }}=11$ ). Kinder, die zu Beginn der vierten Klasse die Strategie nicht mehr einsetzten, wiesen spezifische Merkmale auf, in denen sie den konsistenten Strategen ( $N=33)$ unterlegen waren. Wie Abbildung 5.17 zeigt, war zu Beginn der vierten Klasse ihr Selbstkonzept und ihre Motivation für Lernanforderungen deutlich geringer als bei den Kindern, die die Strategie beibehielten $\left(\mathrm{t}_{\text {Selbstkonzept }}(36)=2,08 ; \mathrm{t}_{\text {Motivation }}(35)=2,08, \mathrm{ps}<\alpha\right)$. 


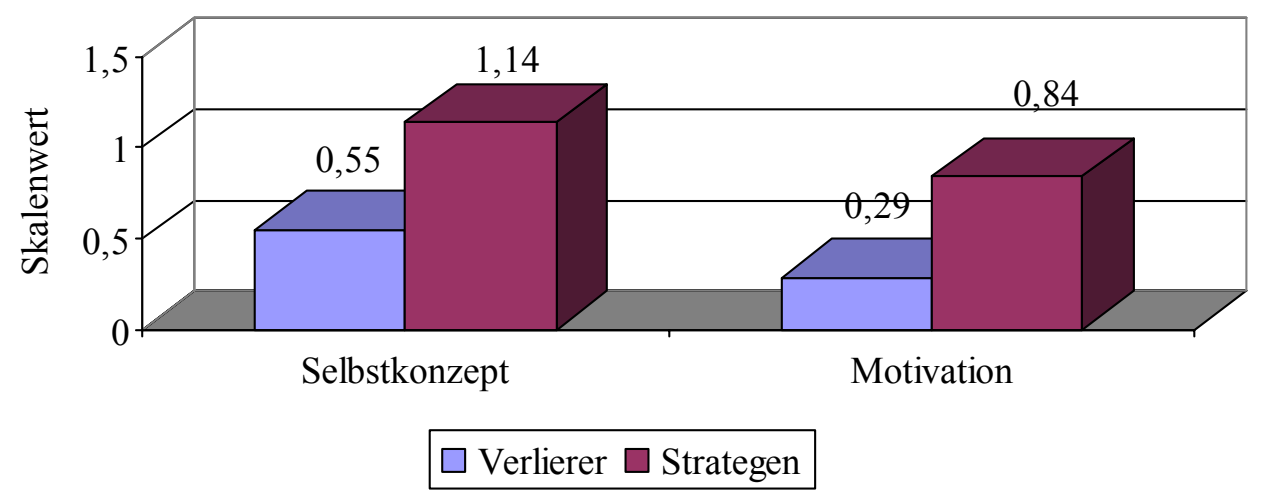

Abbildung 5.17: Mittleres Selbstkonzept und Motivation zu Beginn der vierten Klasse in Abhängigkeit vom Strategenstatus (Strategie-Verlierer und konsistente Strategen zu aufeinanderfolgenden Messzeitpunkten)

\section{Befunde zum kategorialen Organisieren unter Berücksichtigung der Angemessenheit des aufgabenspezifischen Metagedächtnisses}

\section{Strategieproduktion ohne Metagedächtnis}

Ausgangspunkt der folgenden Analysen waren folgende Fragestellungen:

- Verfügen Strategen, die nicht über ein angemessenes Metagedächtnis verfügen, über eine besondere Eigenschaft (z.B. eine hohe Gedächtnisspanne), die sie dazu befähigt?

- $\quad$ Befinden sich Strategen, die noch nicht über ein angemessenes Metagedächtnis verfügen, unmittelbar auf dem Weg dorthin?

Überprüft wurde, worin sich Kinder, die scheinbar auch ohne angemessenes Metagedächtnis die kategoriale Organisationsstrategie bei der Sort-Recall-Aufgabe einsetzen, sich von den Kindern unterscheiden, die dies nicht tun.

Es zeigte sich, dass es unter den Kindern ohne angemessenes Metagedächtnis am Ende der dritten und vierten Klasse deutliche Unterschiede hinsichtlich der Gedächtnisspanne bei strategischen und nicht-strategischen Kindern gab (vgl. Tabelle 5.28). Während die Strategen am Ende der dritten Klasse im Vergleich zu den Nichtstrategen über eine bessere Gedächtnisspanne verfügten ( $\left.\mathrm{t}_{\text {Zahlenspanne rückwärts }}(57)=2,4, \mathrm{p}<\alpha\right)$, kehrte sich das Verhältnis am Ende der vierten Klasse um. Dort verfügten die nichtstrategischen Kinder über eine bessere Gedächtnisspanne ( $\left.\mathrm{t}_{\text {Zahlenspanne vorwärts }}(21)=2,37, \mathrm{p}<\alpha\right)$ 
Tabelle 5.28: Mittlere Gedächtnisspanne (Standardabweichung in Klammern) bei Kindern ohne angemessenes Metagedächtnis

\begin{tabular}{|l|c|c|l|}
\hline & & \multicolumn{2}{|c|}{ Gedächtnisspanne } \\
\hline \multirow{2}{*}{ Ende K1. 3 } & Nichtstrategen $(\mathrm{N}=37)$ & $3,78(0,63)$ & \multirow{2}{*}{ rückwärts } \\
\cline { 2 - 4 } & Strategen $(\mathrm{N}=22)$ & $4,27(0,94)$ & \\
\hline \multirow{2}{*}{ Ende K1. 4 } & Nichtstrategen $(\mathrm{N}=13)$ & $5,69(1,07)$ & \multirow{2}{*}{ vorwärts } \\
\cline { 2 - 4 } & Strategen $(\mathrm{N}=10)$ & $4,8(0,59)$ & \\
\hline
\end{tabular}

Obwohl weder die Strategen noch die Nicht-Strategen das Kriterium erreichten, nach dem ihnen ein angemessenes Metagedächtnis bescheinigt worden wäre, gab es deutliche Unterschiede hinsichtlich der vorhandenen metakognitiven Kompetenzen, besonders am Ende der zweiten und zu Beginn der vierten Klasse (vgl. Tabelle 5.29). So war das Metagedächtnis der Kinder, die sich am Ende der zweiten Klasse $(\mathrm{t}(78)=3,19, \mathrm{p}<\alpha)$ und zu Beginn der dritten Klasse $(\mathrm{t}(69)=3,54, \mathrm{p}<\alpha)$ strategisch verhielten, am Ende der zweiten Klasse deutlich besser als das der Nichtstrategen. Bei Strategen zu Beginn der vierten Klasse war die metakognitive Überlegenheit sogar kontinuierlich ab der dritten Klasse zu beobachten $\left(\mathrm{t}_{\text {Anfang K1.3 }}(51)=2,83\right.$; $\left.\mathrm{t}_{\text {Ende K1.3 }}(51)=2,6 ; \mathrm{t}_{\text {Anfang K1.4 }}(51)=2,46, \mathrm{ps}<\alpha\right)$.

Tabelle 5.29: Durchschnittliches metakognitives Wissen (prozentual richtig beantwortete Items; Standardabweichung in Klammern) am Ende der zweiten Klasse und zu Beginn der vierten Klasse bei Kindern ohne angemessenes Metagedächtnis

\begin{tabular}{|c|c|c|c|}
\hline & Strategentypus & Metakogniti & Wissen $(\%)$ \\
\hline \multirow{2}{*}{ Ende K1. 2} & Nichtstrategen $(\mathrm{N}=71)$ & $27,23(26,62)$ & \multirow{4}{*}{$\begin{array}{c}\text { am Ende der } \\
\text { zweiten Klasse }\end{array}$} \\
\hline & Strategen $(\mathrm{N}=9)$ & $57,41(27,78)$ & \\
\hline \multirow{2}{*}{ Anfang K1. 3} & Nichtstrategen $(\mathrm{N}=56)$ & $24,4(25,81)$ & \\
\hline & Strategen $(\mathrm{N}=15)$ & $52,22(31,4)$ & \\
\hline \multirow{2}{*}{ Anfang K1. 4} & Nichtstrategen $(\mathrm{N}=31)$ & $33,99(29,47)$ & \multirow{4}{*}{$\begin{array}{l}\text { zu Beginn der } \\
\text { vierten Klasse }\end{array}$} \\
\hline & Strategen $(N=22)$ & $54,55(30,83)$ & \\
\hline \multirow{2}{*}{ Ende K1. 4} & Nichtstrategen $(\mathrm{N}=13)$ & $14,1(15,36)$ & \\
\hline & Strategen $(\mathrm{N}=10)$ & $47,5(22,92)$ & \\
\hline
\end{tabular}




\section{Ausbleibende Strategieproduktion trotz angemessenem Metagedächtnis}

Hierzu ließ sich folgende Fragestellung formulieren:

- Zeigen Kinder, die trotz eines angemessenen Metagedächtnisses die Organisationsstrategie nicht einsetzen, eine geringere Motivation und ein niedrigeres Selbstkonzept als die strategischen Kinder?

- Verfügen die nicht-strategischen Kinder, obwohl sie das Kriterium eines angemessenen Metagedächtnisses erreichen, noch nicht über so elaborierte metakognitive Kenntnisse wie die strategischen Kinder?

Zur Überprüfung dieser Annahmen wurden Strategen und Nicht-Strategen mit angemessenem Metagedächtnis miteinander verglichen. Unter den Kindern, die am Ende der dritten Klasse über ein angemessenes Metagedächtnis verfügten, gab es ebenfalls Unterschiede in Abhängigkeit von ihrem Strategenstatus. Strategen waren nicht-strategischen Kindern am Ende der zweiten und am Ende der dritten Klasse in Bezug auf ihr Strategiewissen überlegen ( $\left.\mathrm{t}_{\text {Ende K1.2 }}(25)=2,52 ; \mathrm{t}_{\text {Ende K1.3 }}(25)=2,45, \mathrm{ps}<\alpha\right)$. Die dazugehörigen Mittelwerte und Standardabweichungen sind Tabelle $5.30 \mathrm{zu}$ entnehmen.

Tabelle 5.30: Mittleres metakognitives Wissen (prozentualer Anteil richtig beantworteter Items; Standardabweichung in Klammern) von Strategen und Nicht-Strategen mit angemessenem Metagedächtnis am Ende der dritten Klasse

\begin{tabular}{|c|c|c|}
\hline \multirow{2}{*}{} & \multicolumn{2}{|c|}{ Metakognitives Wissen (\%) } \\
\cline { 2 - 3 } & Ende Klasse 2 & Ende Klasse 3 \\
\hline Nichtstrategen $(\mathrm{N}=11)$ & $31,82(28,34)$ & $68,94(82,81)$ \\
\hline Strategen $(\mathrm{N}=16)$ & $63,54(34,54)$ & $82,81(14,1)$ \\
\hline
\end{tabular}

Auch zu Beginn der vierten Klasse hoben sich strategische Kinder mit angemessenem Metagedächtnis von nicht-strategischen Kindern mit vergleichbarem Wissen ab (vgl. Abbildung 5.18). Ab der dritten Klasse verfügten sie über ein besseres Selbstkonzept ( $\mathrm{t}_{\text {Anfang }}$ $\mathrm{K} 1.3(31)=2,6 ; \mathrm{t}_{\text {Ende K1.3 }}(31)=2,48 ; \mathrm{t}_{\text {Anfang K1.4 }}(31)=2,34$, ps $\left.<\alpha\right)$. Am Ende der dritten Klasse war auch ihre Lernmotivation höher $(\mathrm{t}(31)=2,26, \mathrm{p}<\alpha)$. 


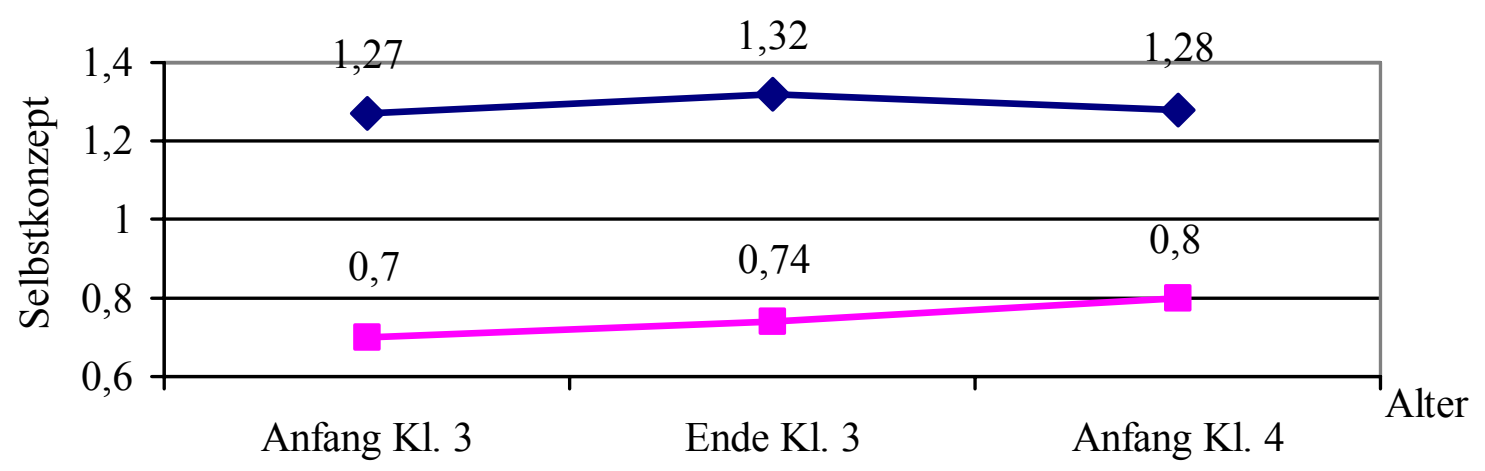

$\rightarrow$ Strategen - Nichtstrategen

Abbildung 5.18: Durchschnittliches Selbstkonzept bei Kindern mit angemessenem Metagedächtnis zu Beginn der 4. Klasse, in Abhängigkeit von ihrem Strategenstatus

Bei den Kindern, die in der vierten Klasse über ein angemessenes Metagedächtnis verfügten, waren Unterschiede in den verbalen Fähigkeiten erkennbar. Dieses äußerte sich darin, dass der Wortschatz von Strategen zu Beginn $(\mathrm{t}(31)=2,12, \mathrm{p}<\alpha)$ und am Ende der vierten Klasse $(\mathrm{t}(57)=2,05, \mathrm{p}<\alpha)$ wesentlich besser war als der von Nichtstrategen, die ebenfalls über ein angemessenes Metagedächtnis verfügten.

\section{Ineffektive Strategieproduktion trotz angemessenem Metagedächtnis}

Hier stellt sich die Frage, ob

- $\quad$ effektive Strategen vor ihrer effektiven Strategieproduktion über ein besseres

Metagedächtnis verfügten als die ineffektiven Strategen und

- ob unter den Kindern mit angemessenem Metagedächtnis die Kinder, die die Strategie ineffektiv einsetzen, eine höhere Motivation zeigen als die effektiven Strategen.

Der Vergleich von effektiven und ineffektiven Strategen mit angemessenem Metagedächtnis zu Beginn der vierten Klasse zeigte Unterschiede im Niveau des aufgabenspezifischen Metagedächtnisses, über das sie am Ende der dritten Klasse verfügten $(\mathrm{t}(21)=2,41, \mathrm{p}<\alpha)$. Mit 67,2\% korrekten Antworten waren die effektiven den ineffektiven Strategen $(37,5 \%)$ deutlich überlegen, d.h. im Gegensatz $\mathrm{zu}$ den ineffektiven Strategen verfügten sie vor der effektiven Strategieproduktion über ein angemessenes Metagedächtnis.

Unter den Kindern mit angemessenem Metagedächtnis am Ende der vierten Klasse ließen die ineffektiven Strategen eine höhere Motivation erkennen als die zu diesem Zeitpunkt 
effektiven Strategen $(\mathrm{t}(33)=2,24, \mathrm{p}<\alpha)$. Dieses lässt sich vor dem Hintergrund verstehen, dass die meisten Kinder in diesem Alter sowohl die Kategorisierungsstrategie produzieren als auch über ein angemessenes Metagedächntis verfügen. Damit kommt dem Phänomen des Nutzungsdefizits eine andere Bedeutung zu (Entwicklungsrückstand) als in einem frühen Entwicklungstadium (Entwicklungsvorsprung). Die Entscheidung, kategorial zu organisieren, auch wenn dieses (für die Kinder erkennbar) nicht mit einem unmittelbaren Nutzen verbunden ist, sollte demnach motivationale Gründe haben. Für diese Interpretation spricht, dass die ineffektiven Strategen tendenziell auch motivierter als Nichtstrategen mit angemessenem Metagedächtnis waren, wenn auch nicht bedeutsam ( $\mathrm{t}(31)=1,7 ; \mathrm{p}=.098)$.

\section{Besonderheiten unter vertrauten Anforderungen (bei hoher kategorialer Typizität der Items) in der Mitte der Grundschulzeit}

Kinder, die am Ende der zweiten Klasse die Kategorisierungsstrategie effektiv einsetzten, verfügten über eine höhere Gedächtnisspanne vorwärts als die ineffektiven Strategen $\mathrm{zu}$ diesem Zeitpunkt $(\mathrm{t}(25)=3,41, \mathrm{p}<\alpha)$. Bei ihrem ersten Strategieeinsatz wiesen Strategieentdecker zu Beginn der dritten Klasse gegenüber Nicht-Strategen eine höhere Gedächtnisspanne rückwärts $(\mathrm{t}(57)=2,03, \mathrm{p}<\alpha)$ und ein besseres Metagedächtnis auf $(\mathrm{t}(57)=2,1, \mathrm{p}<\alpha)$.

Unter den Kindern ohne angemessenes Metagedächtnis am Ende der zweiten Klasse war bei den Kindern, die trotzdem die Strategie produzierten, eine bessere Gedächtnisspanne (rückwärts) erkennbar als bei den Nichtstrategen $(\mathrm{t}(69)=2,08)$. Darüber hinaus schien die Strategieproduktion einen positiven Einfluss auf das spätere motivationale Befinden zu haben. So war ab dem Ende der dritten Klasse ein höheres Selbstkonzept bei den strategischen Kindern beobachtbar, und zwar sowohl bei den Strategen am Ende der zweiten Klasse $\left(t_{\text {Ende K1. } 3}(78)=2,7 ; t_{\text {Anfang K1. } 4}(78)=2,92 ; t_{\text {Ende Kl }}\right.$. $\left.{ }_{4}(75)=3,3, \mathrm{ps}<\alpha\right)$ als auch zu Beginn der dritten Klasse $\left(\mathrm{t}_{\text {Ende Kl. } 3}(69)=2,02 ; \mathrm{t}_{\text {Anfang K1. } 4}\right.$ $\left.(69)=2,98 ; t_{\text {Ende Kl. }}(69)=2,09, \mathrm{ps}<\alpha\right)$. Letztere zeigten am Ende der dritten Klasse außerdem eine wesentlich höhere Lernmotivation als die Nichtstrategen $(\mathrm{t}(68)=2,35$, $\mathrm{p}<\alpha)$.

Besonderheiten unter vertrauten Anforderungen (bei erhöhter Itemanzahl) am Ende der Grundschulzeit

Unter Verwendung einer erhöhten Itemanzahl konnte am Ende der dritten und zu Beginn der vierten Klasse bei der Hälfte der Strategen (45\% bzw. 56,5\%) ein 
angemessenes Metagedächtnis festgestellt werden. Am Ende der vierten Klasse stieg dieser Anteil auf 80,9\%. Allerdings wendete auch ein Drittel der Kinder (21,2-35,6\%), die Strategie trotz des vorhandenen Wissens nicht an.

Als hinreichende Bedingung für die Strategieproduktion am Ende der dritten Klasse konnte das gleichzeitige Vorliegen eines angemessenen Metagedächtnisses und einer überdurchschnittlichen Lernmotivation identifiziert werden, d.h. alle Kinder, die über beide Merkmale verfügten, verhielten sich strategisch.

Ein angemessenes Metagedächtnis schien auch für die effektive Strategieproduktion von zunehmender Bedeutung zu sein. Während am Ende der dritten Klasse der prozentuale Anteil der Kinder mit gutem metakognitiven Wissen unter den nutzungsdefizitären Kindern (50\%) noch etwas höher war als der vergleichbare Anteil unter den effektiven Strategen (42,3\%) änderte sich dieses Verhältnis bis zum Ende der Grundschulzeit. Zu Beginn der vierten Klasse war der prozentuale Anteil vergleichbar (57,1\% vs. 56\%), am Ende der vierten Klasse war der Anteil metakognitiv kompetenter Kinder unter den effektiven Strategen höher (89,3\%) als bei den nutzungsdefizitären Kindern (68,4\%). Dieser Zusammenhang am Ende der vierten Klasse erwies sich allerdings nur auf den 10\%-Niveau als statistisch bedeutsam $(\Phi=.260, \mathrm{p}=.076)$.

Am Ende der vierten Klasse konnten auch auf Individualebene Unterschiede zwischen effektiven und nutzungsdefizitären Strategen gefunden werden. Diese betrafen nicht nur das Metagedächtnis ( $\mathrm{t}(45)=2,4, \quad \mathrm{p}<\alpha)$, sondern auch die Gedächtnisspanne rückwärts $(\mathrm{t}(45)=2,07, \mathrm{p}<\alpha)$.

Darüber hinaus ließen sich charakteristische Unterscheidungsmerkmale zu Beginn der Untersuchung identifizieren. Effektive Strategen am Ende der dritten Klasse ließen im Vergleich zu uneffektiven Strategen am Ende der zweiten Klasse ein höheres Selbstkonzept erkennen $(\mathrm{t}(37)=2,37)$, bei Strategen am Ende der dritten und zu Beginn der vierten Klasse war eine höhere Gedächtnisspanne rückwärts beobachtbar $(\mathrm{t}(38)=2,79$ bzw. $\mathrm{t}(44)=2,83$, ps $<\alpha)$. Wie Abbildung 5.19 zu entnehmen ist, verfügten alle Kinder, die die Strategie ab dem Ende der dritten Klasse effektiv einsetzen, am Ende der zweiten Klasse über eine höhere Gedächtnisspanne vorwärts als Kinder mit einem Nutzungsdefizit $\left(t_{\text {Strategen Ende K1. } 3}(38)=2,29 ; t_{\text {Strategen Anfang K1. } 4}\right.$ $\left.(44)=3,22 ; t_{\text {Strategen Ende Kl. } 4}(45)=2,54, \mathrm{ps}<\alpha\right)$. 


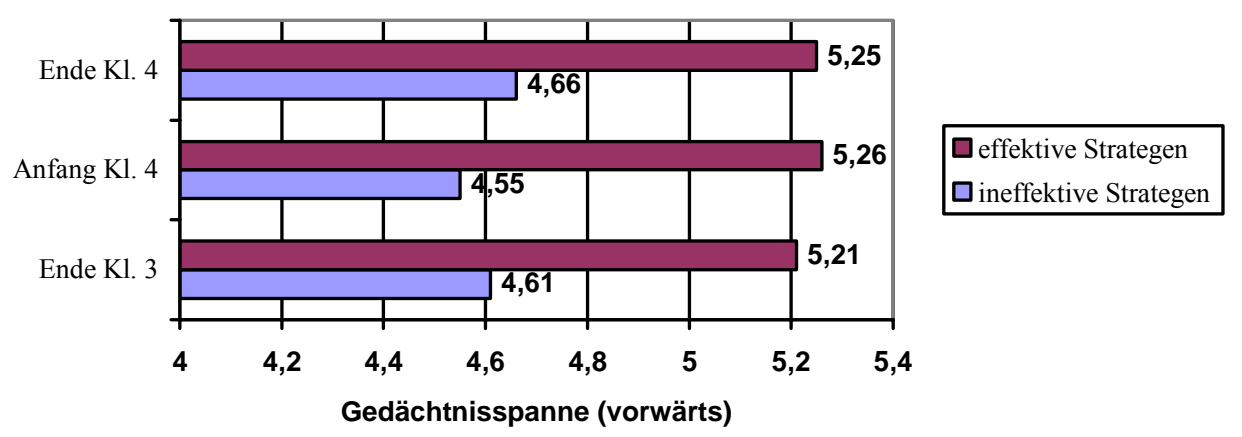

Abbildung 5.19: Mittlere Gedächtnisspanne vorwärts (Ende Kl. 2) in Abhängigkeit von der Effektivität des Strategiegebrauchs und dem Messzeitpunkt

Auch für die Effektivität der ersten Strategieproduktion schien die Gedächtniskapazität von Bedeutung zu sein. Eine überraschende Beobachtung konnte bei den Kindern gemacht werden, die zu Beginn der vierten Klasse das erste Mal die Strategie einsetzen und davon in unterschiedlichem Ausmaß profitieren. Die Kinder, bei denen ein Nutzungsdefizit festgestellt werden konnte, hatten vor ihrer Strategieproduktion eine höhere Gedächtnisspanne (rückwärts) als die effektiven

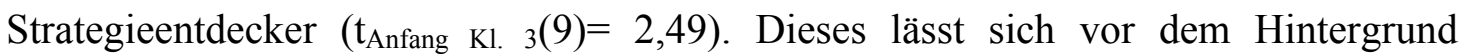
verstehen, dass eine neue Strategie immer in das vorhandene Verhaltensrepertoir integriert werden muss. Bei Kindern mit früher guter kognitiver Leistungsfähigkeit könnten neben der Kategorisierungsstrategie auch andere Formen der Aufgabenbearbeitung zur Verfügung stehen, was zu Interferenzen führt.

Darüber hinaus ließen sich die Annahmen bestätigen, die hinsichtlich der Ursachen für den auch ohne angemessenes Metagedächtnis möglichen Strategieeinsatz (höhere Gedächtnisspanne, Ansätze für metakognitives Wissen) formuliert worden waren. Von den Kindern ohne angemessenes Strategiewissen am Ende der dritten Klasse verfügten die strategischen Kinder im Vergleich zu den Nichtstrategen über eine bessere Gedächtnisspanne vorwärts $\left(\mathrm{t}_{\text {Ende K1.3 }}(57)=2,2, \mathrm{p}<\alpha\right)$ und rückwärts $\left(\mathrm{t}_{\text {Ende }}\right.$ $\mathrm{K} 1.3(57)=3,23, \mathrm{p}<\alpha)$. Zu Beginn der vierten Klasse unterschieden sich die Gruppen in ihrer Gedächtnisspanne vorwärts $(\mathrm{t}(51)=3,39, \mathrm{p}<\alpha)$. Kinder, die am Ende der vierten Klasse auch ohne angemessenes Metagedächtnis die Strategie einsetzten, erwiesen sich zu Beginn und am Ende der vierten Klasse als wesentlich motivierter als Kinder, die sich unstrategisch verhielten $(\mathrm{t}(21)=2,76$ bzw. $\mathrm{t}(21)=3,03, \mathrm{ps}<\alpha)$. Auch wenn das aufgabenspezifische Metagedächtnis dieser Kinder nicht als angemessen bezeichnet 
werden konnte, waren Abstufungen in dem strategischen Wissen der Kinder erkennbar. Am Ende der dritten Klasse verfügten die strategischen Kinder bei ihrer Strategieproduktion über bessere metakognitive Fähigkeiten als die nicht-strategischen Kinder $(\mathrm{t}(57)=2,06, \mathrm{p}<\alpha)$. Strategen zu Beginn der vierten Klasse zeigten zu fast allen Messzeitpunkten bessere metakognitive Fähigkeiten ( $\mathrm{t}_{\text {Ende } \mathrm{K1.2}}(51)=2,18 ; \mathrm{t}_{\mathrm{Anfang}} \mathrm{K1.3}$ $\left.(51)=2,64 ; \mathrm{t}_{\text {Anfang Kl.4 }}(51)=2,34, \mathrm{ps}<\alpha\right)$, Strategen am Ende der vierten Klasse nur zu Beginn der vierten Klasse $(\mathrm{t}(21)=2,55, \mathrm{p}<\alpha)$.

Der Vergleich von Strategen mit und ohne angemessenes Metagedächtnis zeigte, dass am Ende der vierten Klasse die strategischen Kinder mit guten Strategiekenntnissen stets über eine bessere Gedächtnisspanne vorwärts verfügten $\left(t_{\text {Ende Kl.2 }}(45)=3,23 ; t_{\text {Anfang Kl.3 }}(45)=2,25 ; t_{\text {Ende K1.3 }}(45)=2,6 ; t_{\text {Anfang K1.4 }}(45)=3,11, t_{\text {Ende }}\right.$ $\mathrm{K} 1.4(45)=2,36, \mathrm{ps}<\alpha)$.

Der Vergleich von Strategen und Nicht-Strategen mit angemessenem Metagedächtnis lieferte weitere Belege für die bereits in der ersten Liste gemachten Beobachtung, dass eine Strategie machmal trotz vorhandener Kenntnisse aus motivationalen Gründen nicht angewendet wird. Am Ende der dritten Klasse waren auch in der zweiten Liste die Nicht-Strategen weniger motiviert, sich mit Lernanforderungen zu beschäftigen $\left(\mathrm{t}_{\text {Ende K1.3 }}(24)=2,70, \mathrm{p}<\alpha\right)$.

Eine ineffektive Strategieproduktion trotz angemessenem Metagedächtnis schien hingegen eher mit kognitiven Faktoren zusammenzuhängen. Am Ende der dritten Klasse verfügten ineffektive Strategen über eine niedrigere Gedächtnisspanne rückwärts als effektive Strategen $\left(t_{\text {Ende K1.3 }}(16)=2,88, p<\alpha\right)$. Ineffektive Strategen $\mathrm{zu}$ Beginn der vierten Klasse ließen bis zum Beginn der dritten Klasse eine geringere Gedächtnisspanne vorwärts $\left(t_{\text {Ende K1.2 }}(24)=3,1 ; t_{\text {Anfang K1.3 }}(24)=2,71, p s<\alpha\right)$ und ab dem Ende der dritten Klasse eine geringere Gedächtnisspanne rückwärts erkennen ( $t_{\text {Ende K1.3 }}$ $(24)=2,94 ; \mathrm{t}_{\text {Anfang K1.4 }}(24)=2,66$, ps $\left.<\alpha\right)$. Darüber hinaus gab es weitere Hinweise darauf, dass Kinder, die $\mathrm{zu}$ Beginn der vierten Klasse trotz eines angemessenen Metagedächtnisses ein Nutzungsdefizit aufwiesen, noch nicht lange über dieses Strategiewissen verfügten. Wie bereits in der ersten Liste konnte auch für die zweite Liste gezeigt werden, dass das Metagedächtnis der ineffektiven Strategen zu Beginn der dritten Klasse $(\mathrm{t}(24)=2,23, \mathrm{p}<\alpha)$ schlechter war als bei den effektiven Strategen. Dieser Rückstand war hier allerdings auch noch zu Beginn der vierten Klasse erkennbar $\left(\mathrm{t}_{\text {Anfang K1.4 }}(24)=2,3, \mathrm{p}<\alpha\right)$. 


\subsubsection{Zusammenfassung}

In der dritten Klasse ist das zu Beginn der Untersuchung (Ende Klasse 2) vorliegende Metagedächtnis eine bedeutsame Variable, um Unterschiede beim kategorialen Organisieren und der Reproduktionsleistung $\mathrm{zu}$ erklären. $\mathrm{Ab}$ der vierten Klasse lässt sich das Metagedächtnis auf der Basis des vorherigen Sortierverhaltens vorhersagen. Am Ende der vierten Klasse scheint eine wechselseitige Beeinflussung des kategorialen Organisierens und metamemorialen Wissens vorzuliegen.

Unter Berücksichtigung von verschiedenen kognitiven und motivationalen Variablen des gleichen Messzeitpunktes zeigt sich, dass Unterschiede beim effektiven kategorialen Organisieren am Ende der dritten Klasse durch das Metagedächtnis und die Lernmotivation und in der vierten Klasse nur noch durch das Metagedächtnis erklärt werden. Die Einbeziehung von Determinanten aus vorhergehenden Messzeitpunkten macht darüber hinaus deutlich, dass effektives kategoriales Organisieren zu Beginn der dritten Klasse durch das vorausgehende Metagedächtnis und die Gedächtnisspanne (am Ende der zweiten Klasse) beeinflusst wird. Der Einfluss des aktuellen Metagedächtnisses zeigt sich unter diesen Bedingungen noch am Ende der dritten und zu Beginn der vierten Klasse.

Eine Verbesserung des strategischen Verhaltens ohne Beeinflussung der Reproduktionsleistung wird am Ende der vierten Klasse durch die Gedächtnisspanne zu Beginn der Untersuchung (die die Höhe der Arbeitsgedächtniskapaziät widerspiegelt) erreicht. Dieses kann als Hinweis auf eine Determinante für das Zustandekommen einer Form des Nutzungsdefizits verstanden werden.

Die Güte der Gedächtnisleistung ist zu Beginn der dritten und vierten Klasse vor allem auf das kategoriale Organisieren in der Lern- und Abrufphase zurückzuführen, am Ende der dritten und vierten Klasse hingegen nur auf das kategoriale Organisieren in der Lernphase. Als zusätzliche Einflussfaktoren kommen am Ende der zweiten Klasse die Gedächtnisspanne (rückwärts) und in der dritten Klasse das Metagedächtnis und motivationale Faktoren hinzu. Den motivationalen Faktor zu Beginn der dritten Klasse bildet die Lernmotivation, am Ende der dritten Klasse handelt es sich um das Selbstkonzept.

Darüber hinaus ließen sich auch Determinanten für die kategoriale Organisationsstrategie identifizieren. $\mathrm{Zu}$ Beginn der dritten und vierten Klasse bestand ein bedeutsamer Zusammenhang zwischen der bewussten Produktion der kategorialen Organisationsstrategie und der Angemessenheit des Metagedächtnisses. Am Ende der vierten 
Klasse bestand auch ein Zusammenhang zwischen der Strategieeffektivität und der Angemessenheit des Metagedächtnisses. Am Ende der dritten Klasse stellte ein angemessenes Metagedächtnis zusammen mit einer überdurchschnittlichen Gedächtnisspanne (rückwärts) eine hinreichende Bedingung für die Produktion einer effektiven Organisationsstrategie dar. Auch im individuellen Vergleich zeigte sich, dass effektive Strategen ineffektiven Strategen in verschiedenen Merkmalen überlegen sind. Am Ende der dritten Klasse und zu Beginn der vierten Klasse zeichneten sie sich dadurch aus, dass sie zu Beginn der Untersuchung über ein höheres Selbstkonzept verfügten. Effektive Strategen zu Beginn der vierten Klasse verfügten darüber hinaus über eine höhere Gedächtnisspanne (vorwärts) und stets, d.h. auch zu allen vorhergehenden Messzeitpunkten, über ein besseres Metagedächtnis. Effektive Strategen am Ende der vierten Klasse ließen zum Zeitpunkt des Strategiegebrauchs eine höhere Gedächtnisspanne (rückwärts) und ein besseres Metagedächtnis als ineffektive Strategen erkennen. Darüber hinaus verfügten sie bereits zu Beginn der Untersuchung über eine höhere Gedächtnisspanne und ein halbes Jahr zuvor über besseres Strategiewissen.

Um Hinweise auf Determinanten zu erhalten, die eine Bedeutung für den erstmaligen Einsatz der Strategie besitzen, wurde untersucht, in welchen Merkmalen sich Strategieentdecker und Nichtstrategen unterscheiden. $\mathrm{Zu}$ Beginn der dritten Klasse unterschieden sich die Gruppen voneinander. Kinder, die die Strategie zu diesem Zeitpunkt entdeckten, verfügten zu dieser Zeit und bereits davor über ein besseres Metagedächtnis.

Das Aufgeben der Strategie schien demgegenüber eher mit motivationalen Aspekten zusammenzuhängen. Einen Hinweis hierfür lieferte die Beobachtung, dass Strategieverlierer zu Beginn der vierten Klasse eine geringere Motivation und ein niedrigeres Selbstkonzept besaßen als die Kinder, die die Strategie beibehielten.

Das Phänomen, warum auch ohne angemessens Metagedächtnis eine Strategie eingesetzt wird, ließ sich am Ende der dritten und vierten Klasse damit erklären, dass die Kinder über eine bessere Gedächtnisspanne verfügten. Am Ende der zweiten und zu Beginn der vierten Klasse zeigten sie ein besseres Strategiewissen als Nichtstrategen, was vermuten lässt, dass sie sich auf dem Weg zu einem angemessenen Metagedächtnis befanden. Bei Kindern, die zu Beginn der dritten oder am Ende der vierten Klasse die Strategie produzierten, ohne dass gleichzeitig ein angemessenes Metagedächtnis erkennbar war, waren ein halbes Jahr vor diesem Strategiegebrauch bessere Strategiekenntnisse zu beobachten gewesen, so dass eine spätere Verhaltenswirksamkeit vermutet werden kann. 
Der Vergleich von Kindern, die alle über ein angemessenes Metagedächtnis verfügen, aber in unterschiedlichem Maß von der Strategieproduktion profitieren, zeigt, dass effektive Strategen den ineffektiven $\mathrm{zu}$ Beginn der vierten Klasse in ihrem metamemorialen Strategiewissen überlegen sind. Am Ende der vierten Klasse zeigt sich, dass die Kinder, die trotz ihres angemessenen Metagedächtnisses nicht von dem Strategieeinsatz profitieren, wesentlich motivierter sind als die effektiven Strategen (und tendenziell auch als die NichtStrategen), was die Bedeutung des motivationalen Zustandes für die Strategieproduktion in einem (relativ) späten Entwicklungsabschnitt verdeutlicht.

\section{Besonderheiten unter vertrauten Bedingungen}

Am Ende der zweiten Klasse verfügen effektive Strategen über ein besseres Kurzzeitgedächtnis als die ineffektiven Strategen. Kinder, die zu Beginn der dritten Klasse die Strategie zum ersten Mal einsetzen, zeichnen sich durch ein gutes Metagedächtnis und eine hohe Gedächtnisspanne (rückwärts) aus. Eine Strategieproduktion ohne entsprechendes Metagedächtnis scheint bei Kindern möglich zu sein, wenn die Gedächtnisspanne (rückwärts) hoch genug ist.

Am Ende der dritten Klasse bilden ein angemessenes Metagedächtnis und eine überdurchschnittliche Motivation eine hinreichende Bedingung für die Strategenklassifikation. Effektive Strategen zeichneten sich gegenüber ineffektiven Strategen durch eine höhere Gedächtnisspanne zu Beginn der Untersuchung aus. Kinder, die am Ende der dritten Klasse von ihrem Strategiegebrauch profitierten, verfügten darüber hinaus am Ende der zweiten Klasse über ein hohes Selbstkonzept, was auf eine realistische Einschätzung der eigenen Leistungsfähigkeit hindeutet. Effektive Strategen am Ende der vierten Klasse verfügen zum Zeitpunkt der Strategieproduktion über ein besseres Metagedächtnis und eine höhere Gedächtnisspanne (rückwärts) als ineffektive Strategen in dieser Zeit.

Die Effektivität der Strategieanwendung scheint auch in einem Zusammenhang mit der Angemessenheit des Metagedächtnisses am Ende der Grundschulzeit zu stehen. Während in der Gruppe der ineffektiven Strategen der Anteil der Kinder mit angemessenem Metagedächtnis von 50\% auf 68,4\% steigt, erhöht sich der Anteil in der Gruppe der effektiven Strategen von 42,3\% auf 89,3\%. Effektive Strategieentdecker am Anfang der vierten Klasse zeichnen sich gegenüber ineffektiven Strategieentdeckern durch eine bessere Gedächtnisspanne (rückwärts) zu Beginn der dritten Klasse aus. 
Ebenso wie bei der ersten Liste konnte auch bei der zweiten Liste beobachtet werden, dass eine Strategie ohne angemessenes Metagedächtnis eingesetzt bzw. trotz angemessenem Metagedächtnis nicht eingesetzt wurde. Als Ursache kann auch hier von besseren allgemeinen kognitiven Fähigkeiten ausgegangen werden. Am Ende der dritten Klasse verfügten Strategen ohne angemessenes Metagedächtnis über eine höhere Gedächtnisspanne (vorwärts und rückwärts), zu Beginn der vierten Klasse über eine höhere Gedächtnisspanne (vorwärts). Kinder, die zu Beginn der vierten Klasse die Strategie einsetzten ohne über ein angemessenes Strategiewissen zu verfügen, wussten trotzdem mehr über Strategien als Nichtstrategen, d.h. schienen sich auf dem Weg zu einem angemessenen Metagedächtnis zu befinden. Der Nichtgebrauch eine Strategie am Ende der dritten Klasse trotz vorhander Strategiekenntnisse hing mit der geringeren Motivation dieser Kinder zusammen.

Eine ineffektive Strategieproduktion kommt bei Kindern mit angemessenem Metagedächtnis am Ende der dritten Klasse scheinbar durch eine niedrigere Gedächtnisspanne (rückwärts) am Ende der zweiten und zu Beginn der dritten Klasse zustande. Bei Kindern mit angemessenem Strategiewissen zu Beginn der vierten Klasse schien eine unzureichende Strategieeffektivität zusätzlich mit einer geringeren Gedächtnisspanne (vorwärts) am Ende der dritten und zu Beginn der vierten Klasse sowie mit geringeren Strategiekenntnissen (Anfang der 3. + 4. Klasse) zusammenzuhängen.

Auch wenn sich aus den vorliegenden Befunden keine eindeutigen Wirkungszusammenhänge ableiten lassen, so bleibt doch festzuhalten, dass in bestimmten Entwicklungsphasen

- das aufgabenspezifische Metagedächtnis eine entscheidende Bedeutung für das kategoriale Organisieren und seine Effektivität hat

- $\quad$ für die effektive Produktion der bewussten Kategorisierungsstrategie (und besonders für die erste Strategieproduktion bei einer erhöhten Itemanzahl) auch die Gedächtnisspanne eine Rolle spielt

- das Aufgeben einer Strategie in erster Linie auf motivationale Faktoren (geringe Lernmotivation, niedriges Selbstkonzept) zurückzuführen ist

- $\quad$ auch ohne angemessenes Metagedächtnis die Kategorisierungsstrategie eingesetzt wird, woran eine hohe Gedächtnisspanne und Ansätze von metakognitivem Wissen beteiligt sind 
- $\quad$ eine trotz angemessenem Metagedächtnis ausbleibende oder ineffektive

Strategieproduktion damit zusammenhängt, dass die Kinder noch nicht lange über dieses Wissen verfügen oder über eine geringere Gedächtnisspanne verfügen

- $\quad$ eine geringe Motivation bei Kindern mit angemessenem Metagedächnis unterschiedliche Auswirkungen auf den Strategiegebrauch haben kann, d.h. am Ende der dritten Klasse dazu führt, dass die Strategie nicht eingesetzt wird, während es am Ende der vierten Klasse bewirkt, dass sie gerade (trotz geringem Nutzen) eingesetzt wird.

Eine Übersicht der wichtigsten in den verschiedenen Altersstufen identifizierten Determinanten ist den Tabellen 5.30 und $5.31 \mathrm{zu}$ entnehmen. Sofern nicht anders vermerkt handelt es sich bei den genannten Einflussfaktoren um Determinanten des gleichen Messzeitpunktes. 
Tabelle 5.30: Übersicht über die identifizierten Determinanten (Metagedächtnis (MG), Gedächtnisspanne (GS), Motivation und Selbstkonzept) für das kategoriale Organisieren und die Gedächtnisleistung

\begin{tabular}{|c|c|c|c|}
\hline \multicolumn{2}{|l|}{ Verhalten } & Zeitpunkt & Determinanten \\
\hline \multirow{3}{*}{\multicolumn{2}{|c|}{$\begin{array}{ll}\text { Effektives } & \text { kategoriales } \\
\text { Organisieren } & \end{array}$}} & Anfang + Ende Kl. 3; & Metagedächtnis (früher) \\
\hline & & Ende K1. 4 & \\
\hline & & Ende Kl. 3; Anfang Kl. 4 & Metagedächtnis (aktuell) \\
\hline \multirow{5}{*}{\multicolumn{2}{|c|}{ Gedächtnisleistung }} & Ende Kl. 3; Ende Kl. 4 & $\begin{array}{lll}\text { kategoriales } & \text { Organisieren } & \text { in } \\
\text { Lernphase } & & \\
\end{array}$ \\
\hline & & $\begin{array}{l}\text { Ende K1. 2; Anfang Kl. 3; } \\
\text { Anfang K1. } 4\end{array}$ & $\begin{array}{l}\text { kategoriales Organisieren in } \\
\text { Lern- und Abrufphase }\end{array}$ \\
\hline & & $\begin{array}{l}\text { Ende Kl. } 2 \\
\text { Ende Kl. 3- Anf. Kl. } 4 \text { (Liste 2) }\end{array}$ & + Gedächtnisspanne \\
\hline & & $\begin{array}{l}\text { Klasse } 3 \text { (Liste 1) } \\
\text { Ende Kl. } 4 \text { (Liste 2) }\end{array}$ & + Metagedächtnis \\
\hline & & Anfang + Ende Kl. 3 & + Motivation / Selbstkonzept \\
\hline \multirow{8}{*}{$\begin{array}{l}\text { Bewusste } \\
\text { Produktion } \\
\text { der kateg. } \\
\text { Organi- } \\
\text { sations- } \\
\text { strategie }\end{array}$} & \multirow[t]{2}{*}{ Produktion } & Anfang K1. $3+4$ & Angemessenes Metagedächtnis \\
\hline & & Ende Kl. 3 (Liste 2) & $\begin{array}{l}\text { Angem. } M G+\text { überd. Motivation = } \\
\text { hinreichende Bedingung }\end{array}$ \\
\hline & Aufgabe & Anfang K1. 4 & $\begin{array}{ll}\text { (geringe) } & \text { Motivation } \\
\text { Selbstkonzept } & \end{array}$ \\
\hline & \multirow[t]{5}{*}{ Effektivität } & Ende K1. 3 & $\begin{array}{l}\text { Angem. MG + überd. GS (rückw.) } \\
=\text { hinreichende Bedingung }\end{array}$ \\
\hline & & Anfang + Ende Klasse 4 & Metagedächtnis (aktuell) \\
\hline & & Ende Kl. 3 - Anfang Kl. 4 & Selbstkonzept (Ende Kl. 2) \\
\hline & & \begin{tabular}{|l} 
Ende Kl. 4 \\
Ende Kl. 2 (Liste 2)
\end{tabular} & Gedächtnisspanne (Ende Kl. 2) \\
\hline & & Ende Kl. 3- Ende Kl. 4 (Liste 2) & Gedächtnisspanne \\
\hline \multirow{3}{*}{$\begin{array}{l}\text { Erste } \\
\text { Strategie- } \\
\text { produktion }\end{array}$} & \multirow[t]{2}{*}{ Produktion } & Anfang K1. 3 & Metagedächtnis \\
\hline & & Anfang Kl. 3 (Liste 2) & Gedächtnisspanne \\
\hline & Effektivität & Anfang Kl. 4 (Liste 2) & Gedächtnisspanne \\
\hline
\end{tabular}

Legende:

Kursiv $=$ nur in der zweiten Liste relevant

Fett $=$ in beiden Listen relevant 
Tabelle 5.31: Übersicht über die identifizierten Determinanten für das kategoriale Organisieren unter Berücksichtigung der Angemessenheit des Metagedächtnisses

\begin{tabular}{|l|l|l|}
\hline Verhalten & Zeitpunkt & Determinanten \\
\hline \multirow{3}{*}{$\begin{array}{l}\text { Strategiegebrauch ohne } \\
\text { Metagedächtnis }\end{array}$} & $\begin{array}{l}\text { Ende Kl. 3; Ende Kl. 4 } \\
\text { Ende Kl. 2+ Anfang Kl. 4 (Liste 2) }\end{array}$ & Gedächtnisspanne \\
\cline { 2 - 3 } & Ende Kl. 2; Anfang Kl. 4 & Metakognitives Wissen \\
\cline { 2 - 3 } & Anf. + Ende Kl. 4 (Liste 2) & Metakognitives Wissen (früher) \\
\hline \multirow{3}{*}{$\begin{array}{l}\text { Kein Strategiegebrauch } \\
\text { trotz Metagedächtnis }\end{array}$} & Ende Kl. 3 & Metakognitives Wissen \\
\cline { 2 - 3 } & Anfang Kl. 3 - Anf. Kl. 4 & Selbstkonzept (Ende Kl. 2) \\
\cline { 2 - 3 } $\begin{array}{l}\text { Ineffektiver Strategie- } \\
\text { gebrauch trotz } \\
\text { Metagedächtnis }\end{array}$ & Ende Kl. 3 & Motivation \\
\cline { 2 - 3 } & Ende Kl. 4 & Metagedächtnis (früher) \\
\cline { 2 - 3 } & Ende Kl. 3 - Anf. Kl. 4 (Liste 2) & Gedächtnisspanne \\
\cline { 2 - 3 } & Anf. Kl. 4 (Liste 2) & Gedächtnisspanne (früher) \\
\hline
\end{tabular}

Legende:

Kursiv= nur in der zweiten Liste relevant

Fett $=$ in beiden Listen relevant 


\subsection{Exkurs: Geschlechtsspezifische Besonderheiten}

Eine Hauptfragestellung dieser Untersuchung beschäftigte sich mit den interindividuellen Unterschieden bei der Entwicklung der kategorialen Organisationsstrategie und den zugrundeliegenden Determinanten (vgl. Kapitel 3.3). In diesem Zusammenhang wurden in der Vergangenheit auch geschlechtsspezifische Besonderheiten diskutiert (z.B. Cox \& Waters, 1986), weshalb dieser Punkt in der vorliegenden Arbeit nicht übergangen werden soll.

Die Analyse der Leistungen der Kinder, die die kategorialen Strukturen in der SortRecall-Aufgabe in irgendeiner Form genutzt haben (d.h. positive ARC-Werte erzielten), zeigte keine bedeutsamen Geschlechtseffekte hinsichtlich der Höhe des kategorialen Organisierens. Allerdings waren bedeutsame Unterschiede in der Häufigkeitsverteilung von Kindern, die in der ersten Liste überhaupt die kategorialen Strukturen nutzten (ARC $>0$ ), und denen, die dies nicht taten, zu verzeichnen. Aus der Häufigkeitsverteilung (vgl. Tabelle 5.32) wird ersichtlich, dass Mädchen zu Beginn der dritten Klasse in der Lernphase deutlich häufiger die kategoriale Organisationsstrategie einsetzten als die Jungen. Dieses konnte durch nachgeschaltete $\mathrm{t}$-Tests untermauert werden $(\mathrm{t}(84)=2.27, \mathrm{p}<\alpha)$.

Tabelle 5.32: Häufigkeitsverteilung der kategorialen Sortierer in der Lernphase zu Beginn der dritten Klasse, in Abhängigkeit vom Geschlecht

\begin{tabular}{|c|c|c|c|c|}
\hline \multicolumn{2}{|c|}{$\Phi=.362$} & \multicolumn{2}{c|}{ Geschlecht } & \multirow{2}{*}{} \\
\cline { 3 - 5 } \multicolumn{2}{|c|}{} & weiblich & männlich & \\
\hline $\begin{array}{c}\text { Kategoriales Sortieren in } \\
\text { Lernphase }\end{array}$ & Nein & 19 & 25 & 44 \\
\cline { 2 - 5 } & ja & 33 & 9 & 42 \\
\hline \multicolumn{2}{|c|}{} & 52 & 34 & \\
\hline
\end{tabular}

Darüber hinaus zeigte sich, dass Jungen und Mädchen sich auch hinsichtlich der Nutzung der kategorialen Strukturen in der Abrufphase der zweiten Liste am Ende der vierten Klasse unterschieden, also unter Verwendung einer höheren Anzahl an zu lernenden Items, inklusive einer neuen Kategorie (vgl. Tabelle 5.33). 
Tabelle 5.33: Häufigkeitsverteilung der kategorialen Organisierer in der Abrufphase am Ende der 4. Klasse, in Abhängigkeit vom Geschlecht

\begin{tabular}{|l|c|c|c|c|}
\hline \multirow{2}{*}{$\Phi=.292$} & \multicolumn{2}{|c|}{ Geschlecht } & \multirow{2}{*}{} \\
\cline { 2 - 5 } & Nein & 1 & 6 & 7 \\
\hline \multirow{3}{*}{ Sortieren in Abrufphase } & Na & 49 & 26 & 75 \\
\cline { 2 - 5 } & & 50 & 32 & \\
\hline \multicolumn{2}{|l|}{} & ja & & \\
\hline
\end{tabular}

Die varianzanalytische Überprüfung mit dem Geschlecht als unabhängiger Variable und der Reproduktionsleistung als fünffach gestuftem (5 Messzeitpunkte) messwiederholtem Faktor ergab keine systematischen Unterschiede hinsichtlich des Entwicklungsverlaufs der allgemeinen Behaltensleistung von Mädchen und Jungen $(p>\alpha)$. Betrachtet man allerdings die Reproduktionsleistungen $\mathrm{zu}$ den einzelnen Messzeitpunkten, so zeigt sich, dass die Mädchen zu Beginn der dritten Klasse die Jungen in ihrer Behaltensleitung übertrafen $(\mathrm{t}(84)=2,23, \mathrm{p}<\alpha)$. Angesichts der Beobachtung, dass im Gegensatz zu den Jungen die meisten Mädchen zu diesem Zeitpunkt in der Lernphase kategorial organisierten, lassen sich mögliche Rückschlüsse auf die effektive Anwendung der kategorialen Organisationsstrategie beim Enkodieren des Lernmaterials ziehen.

Unabhängig von den bei der Sort-Recall-Aufgabe gezeigten Leistungen zeigte sich, dass die Jungen bis zum Beginn der dritten Klasse über ein deutlich höheres Selbstkonzept verfügten ( $\left.\mathrm{t}_{\text {Ende Kl. } 2}(83)=2,28 ; \mathrm{t}_{\text {Anfang K1. } 3}(84)=3,78, \mathrm{ps}<\alpha\right)$ und ein besseres Allgemeinwissen besaßen $(\mathrm{t}(82)=3,01)$. Demgegenüber war die Lernmotivation der Mädchen am Ende der Grundschulzeit wesentlich höher $\left(\mathrm{t}_{\text {Ende }} \mathrm{Kl.}{ }_{4}(80)=2,97, \mathrm{p}<\alpha\right)$. Im Umgang mit in den in der Versuchssituation gestellten Anforderungen hatten sie sich bereits am Ende der dritten Klasse motivierter gezeigt $(\mathrm{t}(56,1)=2,54, \mathrm{p}<\alpha)$.

Auch wenn man diese Beobachtungen in Bezug zu den Leistungen der Sort-RecallAufgabe setzt, kann höchstens spekuliert werden, dass der zeitlich verzögerte Einsatz der kategorialen Organisationsstrategie der Jungen in Zusammenhang mit ihrem längere Zeit hohen Selbstkonzept steht. Möglicherweise basiert diese hohe Einschätzung der eigenen Kompetenzen auf ihrem guten Allgemeinwissen, das ihnen in anderen Kontexten sicherlich zu einem Leistungsvorteil verholfen hätte. Demgegenüber könnte sich bei Mädchen die Erfahrung der effektiven Bewältigung der gestellten Sort-Recall-Anforderung später noch auf ihre Lernmotivation auswirken, da sie auch noch am Ende der Grundschulzeit eine Bereitschaft signalisieren, sich mit Gedächtnisanforderungen auseinanderzusetzen. 


\section{Diskussion}

Im folgenden Abschnitt sollen zuerst Antworten auf die interessierenden zentralen Fragen dieser Arbeit gegeben werden. Anschließend werden die Befunde unter Berücksichtigung der aktuellen empirischen Datenlage in diesem Forschungsgebiet diskutiert, wobei auf die Arbeiten einiger ausgewählter Autoren (z.B. Sodian \& Schneider, 1999; Schlagmüller \& Schneider, 2002) besonders eingegangen wird. Darüber hinaus wird die Bedeutung der aktuellen Befunde vor dem Hintergrund der Aussagen der StrategieEmergenz-Theorie (Hasselhorn, 1996) reflektiert.

\subsection{Antworten auf die zentralen Fragestellungen}

\section{Wie verläuft die Entwicklung von leistungsdienlichem Kategorisierungs- verhalten?}

Kategoriales Organisationsverhalten kann bereits ab der dritten Klasse bei der Mehrheit der Kinder beobachtet werden. Als bewusste Strategie wird es allerdings erst in der vierten Klasse mehrheitlich angewendet. Der Zeitpunkt der erstmaligen Strategieproduktion liegt bei den meisten Kindern am Anfang der dritten Klasse, die wenigsten beginnen mit der Strategieproduktion erst am Ende der vierten Klasse.

Kategoriales Organisieren scheint grundsätzlich leistungsdienlich zu sein, wobei ältere Grundschüler noch stärker von dem Verhalten zu profitieren scheinen als jüngere. Die Überprüfung der individuellen Nützlichkeit der Strategieproduktion ergab, dass zu den jeweiligen Messzeitpunkten bei ungefähr einem Drittel der Kinder die Leistungssteigerung hinter den Erwartungen zurückbleibt. Bezogen auf die erste Strategieproduktion ist der Anteil ineffektiver Strategen am Ende der dritten und am Ende der vierten Klasse noch höher (2/3).

Unter vertrauten Bedingungen zeigt die Mehrheit der Kinder bereits in der zweiten Klasse kategoriales Organisationsverhalten, auch der Anteil an Strategen ist erhöht. Daran ändert sich auch nichts, wenn die Anzahl an zu lernenden Items erhöht wird. Im Altersvergleich zeigt sich, dass Viertklässler mehr als Zweitklässler von dem kategorialen Organisieren in der Abrufphase profitieren und Sechstklässler gegenüber Grundschülern einen größeren Nutzen aus dem Sortierverhalten ziehen. Dieser Vorteil bleibt gegenüber Viertklässlern auch bestehen, wenn eine größere Itemanzahl verwendet wird. 
Der Umfang des Lernmaterials scheint sich auch auf Individualebene auf die relative Nützlichkeit der Strategieproduktion auszuwirken. Im Vergleich zur ersten Liste lassen sich mehr Kinder identifizieren, die ein Nutzungsdefizit aufweisen.

\section{Welche Determinanten beeinflussen die Produktion und den Nutzen des kategorialen Organisierens?}

Einen besonderen Stellenwert für die Produktion von kategorialem Organisationsverhalten und seine Leistungsdienlichkeit nimmt das Metagedächtnis ein. Am Ende der dritten Klasse scheint darüber hinaus auch die Lernmotivation eine Rolle zu spielen. Unter Berücksichtigung von Variablen aus früheren Messzeitpunkten wird deutlich, dass die am Ende der zweiten Klasse gemessene Gedächtnisspanne und das Metagedächtnis Unterschiede im kategorialen Organisieren und der Reproduktionsleistung zu Beginn der dritten Klasse erklären. Für das kategoriale Organisieren in der Abrufphase (Clustern) bleibt die frühe Arbeitsgedächtniskapazität bis zum Ende der Grundschulzeit eine erklärungsstarke Variable. Eine gute Gedächtnisleistung lässt sich zu jedem Messzeitpunkt am besten durch das kategoriale Organisieren in der Lernphase vorhersagen. Als weitere Einflussfaktoren kommen am Ende der zweiten Klasse die Höhe der Arbeitsgedächtniskapazität und in der dritten Klasse motivationale Faktoren (Lernmotivation und Selbstkonzept) hinzu. Kategoriales Organisieren in der Abrufphase scheint noch nicht am Ende der zweiten Klasse, aber zu Beginn der dritten und vierten Klasse einen positiven Einfluss auszuüben. Die gefundenen Zusammenhänge mit der Arbeitsgedächtniskapazität lassen vermuten, dass es sich beim Clustern um ein relativ ressourcenbelastendes Vorgehen handelt.

Eine hinreichende Erklärung für die Erklärung der Strategieproduktion und seiner Leistungsdienlichkeit bot nie nur eine Variable, sondern die Kombination von Metagedächtnis und einer anderen Determinante. So bildeten ein angemessenes Metagedächtnis und eine überdurchschnittliche Arbeitsgedächtniskapazität eine hinreichende Bedingung für die Strategieproduktion zu Beginn der dritten Klasse unter vertrauten Bedingungen (Liste 2). Am Ende der dritten Klasse stellten ein angemessenes Metagedächtnis und eine überdurchschnittliche Lernmotivation eine hinreichende Bedingung für eine effektive spontane Strategieanwendung (d.h. in der ersten Liste) dar.

Auf Individualniveau konnten folgende interessante Beobachtungen gemacht werden: 
- $\quad$ Kinder, die bereits zu Beginn der dritten Klasse das erste Mal bewusst die kategoriale Organisationsstrategie einsetzten, verfügten über ein besseres Metagedächtnis und einen besseren Wortschatz. Wurde die Strategie zu diesem Zeitpunkt das erste Mal unter vertrauten Bedingungen eingesetzt, so zeichneten sich die Strategen außerdem durch eine bessere Arbeitsgedächtniskapazität aus.

Durch eine hohe Arbeitsgedächtniskapazität am Ende der dritten Klasse zeichneten sich auch die Kinder aus, die die Kategorisierungsstrategie das erste Mal in der zweiten Liste zu Beginn der vierten Klasse effektiv einsetzten.

- $\quad$ Kinder, die zwischen dem Ende der dritten und dem Beginn der vierten Klasse die kategoriale Organisationsstrategie effektiv einsetzten, wiesen am Ende der zweiten Klasse ein höheres Selbstkonzept auf.

- $\quad$ Kinder, die in der vierten Klasse vom kategorialen Organisieren profitierten, verfügten bereits ein halbes Jahr zuvor über bessere strategische Kenntnisse (Metagedächtnis) als Kinder mit einem Nutzungsdefizit. Bei effektiven Strategen zu Beginn der vierten Klasse waren sogar schon am Ende der zweiten Klasse bessere metakognitive Kompetenzen erkennbar.

- $\quad$ Bei Kindern, die am Ende der vierten Klasse (immer noch) keinen Leistungsvorteil durch die Strategieproduktion hatten, waren im Vorfeld (Ende der zweiten und dritten Klasse) Hinweise auf eine geringere Gedächtniskapazität gefunden worden.

\section{Warum profitieren einige Kinder trotz angemessenem Metagedächtnis nicht von dem Strategiegebrauch?}

Wenn Kinder trotz ihres angemessenen Metagedächtnisses nicht von der kategorialen Organisationsstrategie profitieren, wenn eine erhöhte Itemanzahl verwendet wird (Liste 2), so hängt dieses unter anderem mit ihrer allgemeinen kognitiven Leistungsfähigkeit zusammen. Dieses äußert sich bei den nutzungsdefizitären Strategen am Ende der dritten Klasse in einem schlechteren Arbeitsgedächtnis vor der Strategieproduktion (Ende K1. 2 - Anf. K1. 3), und bei den nutzungsdefizitären Kindern $\mathrm{zu}$ Beginn der vierten Klasse in einem schlechteren Kurzzeitgedächtnis (Ende K1. 3- Anf. K1. 4). Darüber hinaus scheint auch ihr Metagedächtnis (Anf. Kl. $3+4)$ nicht dieselbe Qualität zu besitzen wie bei Strategen.

Kinder, die am Ende der vierten Klasse über ein angemessenes Metagedächtnis verfügen und sich spontan (d.h. in der ersten Liste) strategisch verhalten, aber keinen 
Leistungsvorteil haben, zeichneten sich durch eine erhöhte Lernmotivation aus (im Vergleich zu effektiven Strategen).

\section{Warum können Kinder auch ohne angemessenes Metagedächtnis die kategoriale Organisationsstrategie anwenden?}

Obwohl bei dieser Gruppe von Strategen das Metagedächtnis noch nicht als angemessen bezeichnet werden kann, verfügen sie über ein höheres Maß an strategischen Kenntnissen als Nichtstrategen. Hinweise darauf finden sich entweder zum gleichen Messzeitpunkt (bei Strategen am Ende der zweiten und zu Beginn der vierten Klasse) oder ein halbes Jahr zuvor (bei Strategen zu Beginn der dritten und am Ende der vierten Klasse).

Für die Strategieproduktion ohne angemessenes Metagedächtnis scheint eine hohe Arbeitsgedächtniskapazität wichtig zu sein. Belege für diese Annahme finden sich bei der spontanen Strategieproduktion (Liste 1) am Ende der dritten Klasse, unter vertrauten Bedingungen (Liste 2) darüber hinaus auch am Ende der zweiten Klasse. Kinder, die die Strategie dort auch ohne angemessenes Metagedächtnis produzierten, hoben sich von Nichtstrategen durch eine bessere Arbeitsgedächtniskapazität ab. Die Befunde sprechen weiterhin dafür, dass diese Aussage auch unter schwierigeren Aufgabenbedingungen zutrifft. Unter Verwendung einer höheren Itemanzahl scheinen Strategen die kategoriale Organisationsstrategie ohne angemessenes Metagedächtnis anwenden zu können, wenn sie über eine hohe Kurzzeitgedächtniskapazität verfügen.

\section{Warum geben Kinder die kategoriale Organisationsstrategie wieder auf?}

Die Argumentation, dass Kinder eine Strategie bewusst nicht mehr einsetzen, wenn sie erkennen, dass das Verhalten nicht mit einem Leistungsvorteil verbunden ist, wird durch die Beobachtung entkräftet, dass bis zur dritten Klasse alle Kinder, die in der ersten Liste nicht von der Strategie profitierten, in der zweiten Liste erneut das gleiche Verhalten zeigten. Erst am Ende der vierten Klasse gibt es vermehrt Kinder, die die Strategie in der zweiten Liste nicht einsetzen, wenn sie zuvor keinen Nutzen von der Strategieproduktion hatten.

Für das Aufgeben der Strategie scheinen eher motivationale Gründe verantwortlich zu sein. Dafür spricht u.a. die Beobachtung, dass Kinder, die zu Beginn der vierten Klasse die Strategie aufgeben über eine geringere Lernmotivation und ein niedrigeres Selbstkonzept verfügen als Kinder, die die Strategie beibehalten. 
Die Lernmotivation scheint auch einen Anteil daran zu haben, wenn Kinder die kategoriale Organisationsstrategie nicht anwenden, obwohl sie über das erforderliche Wissen verfügen. So erwiesen sich am Ende der dritten Klasse die Kinder, die das Lernmaterial in der zweiten Liste trotz angemessenem Metagedächtnis nicht kategorial ordneten, als wesentlich weniger motiviert, als die Kinder, die sich strategisch verhielten.

\subsection{Einordnung der eigenen Befunde in die empirische Datenlage}

\section{Entwicklung der kategorialen Organisationsstrategie}

Aufgrund der in Querschnittuntersuchungen gefundenen Altersunterschiede wurde lange Zeit von einem kontinuierlichen Entwicklungsverlauf beim Erwerb des kategorialen Organisierens ausgegangen (Moely et al., 1969). Längsschnittliche Befunde lieferten hingegen Hinweise auf einen eher sprunghaften Erwerb dieser Strategie (Sodian \& Schneider, 1999). Grundlage für diese Aussage bildete die Messung der strategischen Kompetenz der Kinder im Abstand von zwei Jahren über die Bearbeitung einer Sort-Recall-Aufgabe. Sodian und Schneider (1999) beobachteten dabei, dass zwischen dem 4. und 12. Lebensjahr 81\% der Kinder von „unstrategischem“ Verhalten (RR-Werte <.30) den Sprung zu einer fast perfekten Sortierleistung schafften, d.h. mindestens $80 \%$ der Items entsprechend der vorliegenden Kategorien anordneten. Obwohl viele Kinder bereits sehr früh ein fast perfektes Niveau an konzeptuellem Organisieren erreichten (40\% der 4- bis 6-jährigen), zeigte sich im weiteren Entwicklungsverlauf, dass 70\% dieser frühen Strategen die Strategie wieder aufgaben und erst zu einem späteren Zeitpunkt wieder einsetzten. Aufgrund des großen Anteils an Strategieverlierern ist fraglich, ob diese Kinder wirklich die kategoriale Organisationsstrategie entdeckt haben oder ob sie einfach ein bestimmtes Verhalten ausprobiert haben, ohne dabei an die Konsequenzen zu denken. Ab dem 8. Lebensjahr scheint das Verhalten der Kinder eine größere Konstanz zu bekommen, was für ein zielgerichtetes Verhalten bei der Aufgabenbearbeitung spricht. In der LOGIK-Studie (Sodian \& Schneider, 1999) setzen von den späten Strategieentdeckern (ab dem 8. Lebensjahr) 24\% die Strategie das erste Mal im Alter von 8 Jahren, 21\% im Alter von 10 Jahren und 5\% mit 12 Jahren ein. Von diesen Kindern behielten $65 \%$ die Strategie bei, $21 \%$ folgten einem U-förmigen Verlauf (Strategiegebrauch im Alter von 8 Jahren, keinen Strategiegebrauch im Alter von 10 Jahren und Strategiegebrauch im Alter von 12 Jahren), und 14\% gaben die Strategie für den Untersuchungszeitraum endgültig auf. 
Auch in der vorliegenden Untersuchung erwies sich der Großteil der Kinder ab der dritten Klasse als strategisch. Über den gesamten Untersuchungszeitraum wendeten $22 \%$ der Kinder die kategoriale Organisationsstrategie kontinuierlich an, 9,8\% der Kinder gaben die Strategie endgültig auf. Der Anteil der Veränderer, d.h. der Kinder, die sie uneinheitlich einsetzten, lag bei 40,2\%. Im Vergleich zur Studie von Sodian und Schneider (1999), in der 65\% der Kinder die Strategie aufrechterhielten, 14\% die Strategie aufgaben und 21\% einen U-förmiger Verlauf zeigten, spiegelt dieses Muster eine höhere Heterogenität des Strategiegebrauchs wieder. Im halbjährlichen Abstand war die Konsistenz des strategischen Verhaltens deutlich höher, d.h. die meisten Kinder behielten die Strategie $\mathrm{zu}$ aufeinanderfolgenden Messzeitpunkten bei.

In der mikrogenetischen Studie von Schlagmüller und Schneider (2002) wurde die Strategie ebenfalls von dem Großteil der Kinder über die meisten Sitzungen beibehalten. Der Erwerb dieser Strategie schien sich aber wie bei Sodian und Schneider (1999) auch im mikrogenetischen Design eher sprunghaft als kontinuierlich zu vollziehen (Schlagmüller \& Schneider, 2002). Eine Differenzierung zwischen dem kategorialen Organisieren in der Lernund Abrufphase zeigt allerdings, dass sich dieser Befund in erster Linie auf die Sortierstrategie in der Lernphase und weniger auf das Verhalten in der Abrufphase bezieht. Letzteres scheint sich weniger abrupt zu vollziehen. Schlagmüller und Schneider (2002) beobachteten, dass eine abrupte Verbesserung der Sortierleistung nicht von einer entsprechenden Verbesserung der Clusterleistung begleitet wird. Während beim Sortieren häufig Extremwerte verzeichnet wurden (beim Sortieren: 40\% Werte unter .1 und 55\% Werte über .9), lagen die Clusterwerte auf einem Kontinuum (beim Clustern: 30\% über .9 und 55\% zwischen .1 und .9).

Auch die Befunde der vorliegenden Untersuchung sprechen dafür, dass sich das Sortierverhalten abrupter und schneller verbessert als das Clustern beim Informationsabruf. Am Ende der zweiten Klasse gibt es wenige Kinder, die die kategorialen Strukturen bereits in der Lernphase nutzen (25,6\%). In der Abrufphase ist der Anteil deutlich höher (48,8\%). Im weiteren Verlauf steigert sich der Anteil in der Lernphase erheblich (48,8\% zu Beginn, 62,8\% am Ende der dritten Klasse) und liegt zu Beginn der vierten Klasse bei 80,2\%. Der Prozentsatz der kategorialen Organisierer nimmt bis zu diesem Zeitpunkt ebenfalls zu (82,6\%). Am Ende der vierten Klasse steigt er allerdings noch weiter (auf 91,5\%), während der Anteil der Sortierer wieder zurückgeht (69,5\%).

Die Klassifikation der Strategen wurde in der Vergangenheit entweder anhand des Verhaltens in der Lern- oder der Abrufphase vorgenommen. Viele Autoren vertraten die 
Auffassung, dass eher das Sortierverhalten während der Lernphase als das Clustern während des Abrufs Hinweise auf einen bewussten Strategieeinsatz liefert (vgl. Bjorklund, 1987; Hasselhorn, 1992; Ornstein, Baker-Ward \& Naus, 1988; Schneider, 1986). Eine Bestätigung dieser Annahme sahen Schlagmüller und Schneider (2002) darin, dass Sortierer zwar den Nicht-Sortierern überlegen waren, nicht aber die Clusterer den Nicht-Clusterern. Umgekehrt nutzen in der Studie von Coyle und Bjorklund (1996) nur wenige der Clusterer in der Abrufphase auch in der Lernphase eine Sortierstrategie.

Das Zustandekommen hoher Clusterwerte wurde daher eher auf automatische Prozesse zurückgeführt, bei denen die Assoziationen zwischen den zu lernenden Items genutzt werden. Unterstützt wird diese Annahme dadurch, dass die Cluster-Werte im Allgemeinen für die Nicht-Sortierer (mittlerer $\mathrm{ARC}=.52$ ) hoch ausfielen und sich nicht wesentlich von denen der Sortierer (mittlerer $\mathrm{ARC}=.63$ ) unterschied (Schlagmüller \& Schneider, 2002). Dabei ist allerdings zu bedenken dass in beiden Phasen Einflussfaktoren wirksam werden können, die zu einer Überschätzung der strategischen Kompetenzen führen. Während in der Abrufphase Organisationsprozesse auch aufgrund automatischer Aktivierungsprozesse (d.h. unterschiedliches Vorwissen) zustande kommen können, kann in der Lernphase die Manipulation am Lernmaterial durch Kontexteinflüsse (Instruktion, Feedback des Versuchsleiters) provoziert werden.

Außerdem sind das Verhalten in der Lernphase und das in der Abrufphase nicht unabhängig voneinander. Dieses zeigt sich auch in der vorliegenden Untersuchung an der hohen Gesamtkorrelation von $r \geq .46 \quad(p<\alpha)$. Als Kriterium für einen vollständigen Strategiegebrauch scheint es daher angebracht zu sein, sowohl das Verhalten in der Lernphase als auch das in der Abrufphase zu berücksichtigen (vgl. Hasselhorn, 1996). Unter diesem Kriterium erweisen sich erst in der vierten Klasse die meisten Kinder als strategisch (52,3\% zu Beginn, 51,2\% am Ende). Am Ende der zweiten Klasse liegt der Prozentsatz bei 10,5\% und erhöht sich im Laufe der dritten Klasse von 29,1\% auf 44,2\%.

\section{Effektivität der kategorialen Organisationsstrategie}

Ein wesentliches Kriterium bei der Nutzungsdefizitdiskussion ist die Angemessenheit des Strategieeinsatzes, unter dem normalerweise wirklich eine Leistungssteigerung $\mathrm{zu}$ erwarten ist (vgl. Schlagmüller \& Schneider, 2002; Waters, 2000). Wie in Kapitel 2.2 dargestellt, wurde von vielen Autoren eine mit dem Alter zunehmende Verbindung von konzeptuellen Organisationsstrategien und der Wiedergabeleistung berichtet (vgl. Hasselhorn, 1990; Kee \& Bell, 1981; Schneider, 1986), was allgemein auf eine Verbesserung der 
Strategieeffektivität schließen lässt. Diesen Befunden stehen andere Studien gegenüber, die über eine geringe Leistungsdienlichkeit der Strategie berichten (vgl. Miller \& Seier, 1994). Unterschiedliche Befunde hinsichtlich der Effektivität des strategischen Verhaltens waren nicht nur auf unterschiedliche experimentelle Designs sondern auch auf die Wahl unterschiedlicher Indizes für die Strategenklassifikation zurückzuführen (z.B. Coyle \& Bjorklund, 1996; Schlagmüller \& Schneider, 2002). Coyle und Bjorklund (1996) fanden unter Fokussierung auf strategisches Verhalten in der Abrufphase sowohl bei Zweit- und Drittklässlern als auch bei Viertklässlern Hinweise auf ein vorhandenes Nutzungsdefizit. In ihrer „modifizierten mikrogenetische Studie“, bei der in einer einzigen experimentellen Sitzung 5 Sort-Recall-Durchgänge durchgeführt wurden, verbesserte sich nur der Strategiegebrauch, aber nicht die Wiedergabeleistung.

Wurde die Klassifikation anhand der Sortierleistung in der Lernphase vorgenommen, so ergab sich ein anderes Bild. Sodian und Schneider (1999; Schneider \& Sodian, 1997) konnten nur bei jüngeren Kindern (unter 8 Jahren) eine geringe Strategieeffektivität feststellen. Dieses zeigte sich sowohl im Vergleich zur Leistung der Gesamtstichprobe, als auch im Vergleich mit den Nicht-Strategen und an den geringen korrelativen Zusammenhänge zwischen Strategiegebrauch und Reproduktionsleistung (bei Vierjährigen: $\mathrm{r}=.25$ ). Bei älteren Kindern war der Strategieeinsatz stets mit einer Leistungssteigerung verbunden (d.h. zwischen dem 8. und 10. Lebensjahr doppelt so hohe Leistungssteigerung der Strategen im Vergleich zur Gesamtstichprobe), was sich auch in hohen Korrelationen zwischen Strategie und Reproduktion wiederspiegelte (bei Zehnjährigen: $\mathrm{r}=.70$ ). Regressionsanalytische Auswertungen der Entwicklungsverläufe in der LOGIK-Studie zeigten bei 2/3 der Kinder eine kontinuierliche Verbesserung der Abrufleistung, wobei bei $40 \%$ der Kinder zwischen aufeinanderfolgenden Messzeitpunkten Leistungssprünge zu verzeichnen waren. Davon entfiel die eine Hälfte auf die Zeit zwischen dem 8. und 10. Lebensjahr, die andere Hälfte auf den Zeitraum vom 10. bis 12. Lebensjahr.

Der Nutzen der kategorialen Organisationsstrategie konnte auch in noch kürzeren Untersuchungsabständen unter Beweis gestellt werden; so z.B. in der mikrogenetischen Studie von Schlagmüller und Schneider (2002). Die erste Anwendung der Organisationsstrategien verbesserte bei den meisten der 9- bis 10jährigen Kindern sofort die Reproduktionsleistung. Eine differenzierte Betrachtung der Befunde zeigte, dass die konsistent sortierenden Kinder die inkonsistent sortierenden Kinder in ihrer Erinnerungsleistung übertrafen. Zwischen Clusterern und Nicht-Clusterern waren hingegen keine Reproduktionsunterschiede zu beobachten. 
Auch in der hier vorgestellten Untersuchung erwies sich das strategische Verhalten der 8- bis 10jährigen als effektiv, wobei der korrelative Zusammenhang zwischen kategorialem Organisieren und Reproduktionsleistung bereits bei den Neunjährigen sehr hoch ausfiel. Diese Zusammenhänge zwischen Verhaltensqualität und Reproduktionsleistung traten sowohl bei der spontanen Aufgabenbearbeitung (Korrelation über die Messzeitpunkte vom Beginn der dritten bis zum Ende der vierten Klasse; Sort: $r=.62-.70$; Clustern: $r=.59-.64$ ) als auch unter vertrauten Bedingungen mit kategorial typischem Material (Sort: $r=.75$; Clustern: $\mathrm{r}=.61$ ) und mit einer höheren Itemanzahl auf (Sort: $r=.59-.68$; Clustern: $r=.50-.62$ ).

Die vorliegende Untersuchung bestätigt demnach weitgehend die Befunde aus den Studien von Hasselhorn (1996), der LOGIK-Studie (Schneider \& Sodian, 1997; Sodian \& Schneider, 1999) und der mikrogenetischen Studie von Schlagmüller und Schneider (2002).

\section{Determinanten einer effektiven kategorialen Organisationsstrategie}

Für einen ausbleibenden oder zu geringen Nutzen des strategischen Verhaltens werden sowohl Kontexteinflüsse als auch eine Reihe anderer kognitiver und motivationaler Faktoren diskutiert (vgl. Blöte, Resing, Mazer \& Van Noort, 1999; Miller \& Aloise-Young, 1996). Leider existiert bisher kein theoretisches Rahmenwerk, das die intraindividuellen Veränderungen und interindividuelle Unterschiede hinreichend erklären kann (vgl. Miller, 2000; Miller \& Seier, 1994). Dabei stellt sich die Frage, wie sich das Kontinuum der Strategieentwicklung zum Kontinuum der Strategie-Effektivität (z.B. Abrufvorteile oder anderen Leistungen) verhält und wie hoch die Kosten und der Nutzen einer spontanen Strategieproduktion im Hinblick auf das weitere Vorgehen sind. Bekannt ist, dass die Anstrengungen bei der Strategieproduktion das Auftreten eines Nutzungsdefizit beeinflussen (z.B. Bjorklund \& Harnishfeger, 1987, Experiment 2; Miller, Seier, Probert \& Aloise, 1991). Dieses weist darauf hin, dass sowohl kognitive als auch motivationale Faktoren einen Einfluss auf den spontanen Einsatz von Strategien und die Entwicklung des effektiven Umgangs mit Strategien haben. Diese Wirkung ist natürlich stets im Zusammenhang mit den realisierten Kontextbedingungen zu sehen. Der Stellenwert der Selbständigkeit beim Strategiegebrauch für die nachfolgenden Informationsverarbeitungsprozesse und damit für das Nutzungsdefizit wird noch kontrovers diskutiert (vgl. Miller, 2000; Waters, 2000). Grundsätzlich kann die Effektivität der gezeigten kognitiven Fähigkeiten entlang einer weiten Spanne von Situationen variieren, in denen die soziale und physikalische Unterstützung verändert wird. Trainingsstudien konnten beispielsweise zeigen, dass die Leistungsdienlichkeit der Strategien auf den Trainingszeitraum beschränkt war, während die Strategien selber auch noch später 
„spontan“ eingesetzt wurden (z.B. Bjorklund \& Coyle, 1995). Starke kontextuelle Unterstützung kann demnach die Strategieproduktion fördern, aber nicht notwendigerweise die Strategieeffektivität.

Sodian und Schneider (1999) untersuchten die Bedeutung von basalen Fähigkeiten, verbalen Kompetenzen, Intelligenzunterschieden und strategischem Verhalten für die Gedächtnisleistung mit Hilfe eines hierarchischen linearen Modells (vgl. Bryk \& Raudenbush, 1992). Im Alter von 8 Jahren hing bei den Kindern, die keine Deckeneffekte zeigten, die Reproduktionsleistung mit ihrem Verbal-IQ und ihrem Strategieeinsatz in dieser Zeit zusammen. Darüber hinaus bestand ein Zusammenhang mit der Steigerung der Sortierleistung und Wiedergabeleistung. Demnach spielen nicht nur statische, sondern auch dynamische Prozesse eine Rolle. Da mit diesem Modell 72\% der Varianz des Eingangsniveaus und 48\% der Varianz der Wachstumsraten erklärt werden konnten, wurde geschlussfolgert, dass im Grundschulalter die Reproduktionssteigerungen am besten durch den vermehrten Strategiegebrauch vorhergesagt werden können. Andere Faktoren wie das Metagedächtnis und die Arbeitsgedächtniskapazität schienen nicht ins Gewicht zu fallen (Sodian \& Schneider, 1999).

Auch Hasselhorn (1996) fand in seinen Studien keine Hinweise darauf, dass bei Achtjährigen das aufgabenspezifische Metagedächtnis oder die Arbeitsgedächtniskapazität das kategoriale Organisieren und die Reproduktionsleistung beeinflussen. Als entscheidende Determinante in diesem Altersabschnitt kristallisierte sich hingegen das Vorwissen der Kinder hinaus. Die von ihm in der Strategie-Emergenz-Theorie postulierten Wirkungszusammenhänge konnten erst bei zwei Jahre älteren Kindern beobachtet werden.

„Stattdessen findet sich bei den Viertklässlern der postulierte strategische „Ketten“mechanismus, bei dem davon ausgegangen wird, dass das aufgabenspezifische Metagedächtnis das Erkennen der kategorialen Listenstruktur erleichtert, dieses wiederum das Ausmaß kategorialen Organisierens begünstigt, und schließlich das kategoriale Organisieren auch zu besseren Reproduktionsleistungen führt.“ (Hasselhorn, 1996, S. 162-163).

Der Einfluss des Metagedächtnisses auf den effektiven Strategieeinsatz wurde über das Erkennen der Listenstruktur vermittelt. Die Höhe der Reproduktionsleistung hing dabei nicht ausschließlich vom Ausmaß der kategorialen Organisation ab, sondern wurde darüber hinaus von der Arbeitsgedächtniskapaziät mitbestimmt. Den fehlenden Zusammenhang zur Strategieproduktion interpretierte Hasselhorn (1996) als weiteren Beleg für seine Theorie und gegen die Wissensposition von Bjorklund (1987). 
Angesichts der von ihm selber formulierten Erklärungsgrenzen der StrategieEmergenz-Theorie bleibt allerdings die Frage offen, ob die Aussage, „dass die Kapazität des Arbeitsgedächtnisses ... für das kategoriale Organisieren älterer Grundschulkinder von eher untergeordneter Bedeutung ist“ (Hasselhorn, 1996, S. 164) generell Gültigkeit besitzt oder an bestimmte Voraussetzungen gebunden ist. Unter bestimmten Rahmenbedingungen kann durchaus vermutet werden, dass die Arbeitsgedächtniskapazität und ein gutes Metagedächtnis über die Anpassung des Verhaltens an die konkreten Anforderungen zu einem effektiven Strategieeinsatz beitragen.

Die Befunde der vorliegenden Studie zeigen, dass das Metagedächtnis bereits bei Neunjährigen einen bedeutsamen Einfluss auf das kategoriale Organisieren und seine Leistungsdienlichkeit haben kann. Insgesamt weisen die Ergebnisse darauf hin, dass für die qualitative und quantitative Verbesserung der kategorialen Organisationsstrategie hauptsächlich kognitive und metakognitive Faktoren verantwortlich sind. Das Aufgeben einer Strategie scheint hingegen nicht mit dem ausbleibenden Nutzen der Strategie und auch nicht mit fehlendem metakognitiven Wissen, sondern mit motivationalen Faktoren zusammenzuhängen. Die subjektiv geringe Einschätzung der eigenen Leistungsmöglichkeiten scheint bei diesen Kindern dazu zu führen, dass sie das Vertrauen in den Nutzen des Strategiegebrauchs verlieren und in Folge dessen das bisher gezeigte Verhalten nicht mehr zeigen, obwohl sie objektiv gesehen vorher davon profitiert haben.

\section{Kontextabhängigkeit der kategorialen Organisationsstrategie}

In den Untersuchungen von Hasselhorn (1996) hatte sich gezeigt, dass Viertklässer ihre kategoriale Organisationskompetenz in Aufgaben mit unterschiedlichem Schwierigkeitsniveau (,Sort-Recall“"- und „Free-Recall“-Aufgaben) unterschiedlich oft, aber in unveränderter Qualität einsetzen. Die Kinder, die die kategoriale Organisationsstrategie einsetzten, ließen dabei metamemoriales Bewusstsein erkennen und profitierten von ihrem Verhalten. In der vorliegenden Untersuchung wurde das Schwierigkeitsniveau der zu bearbeitenden ,Sort-Recall“-Aufgabe verändert, indem am Ende der Grundschulzeit in einem zweiten Durchgang die Anzahl der zu lernenden Items erhöht wurde. Unter diesen Bedingungen nahm die Anzahl der Kinder, die die Strategie bewusst einsetzten, nicht ab. Allerdings erhöhte sich der Anteil der Kinder, bei denen ein ineffektiver Strategiegebrauch beobachtbar war. Aussagen über das Schwierigkeitsniveau einer Aufgabe und die damit verbundenen Konsequenzen für die Strategieproduktion und -effektivität erfordern demnach 
die Berücksichtigung der Vielzahl an Kontextfaktoren, die in ihrer Summe den Schwierigkeitsgrad einer Aufgabe festlegen. Die subjektive Schwierigkeit einer Aufgabe hängt darüber hinaus von zahlreichen internen Faktoren ab. Dazu kommt auch das Ausmaß an Erfahrungen im Umgang mit kategorialen Organisationsanforderungen, das beispielsweise bei den Viertklässlern in der hier vorliegenden Studie höher ist als bei den Viertklässlern in der Studie von Hasselhorn (1996).

Die Bedeutung der kategorialen Typizität der Items, auf die Hasselhorn (1996) hinwies, zeigt sich in der vorliegenden Untersuchung im Zusammenhang mit Vertrautheit der Anforderungen zu Beginn der dritten Klasse. Aufgrund der Konfundierung der Einflüsse lässt sich nur allgemein konstatieren, dass unter vereinfachten Bedingungen bereits in der Mitte der Grundschulzeit ein erhöhter Anteil an Kindern die kategoriale Organisationsstrategie einsetzt und davon in Form einer Leistungssteigerung profitiert.

Die Veränderungen am Lernmaterial, die Sodian und Schneider (1999) zwischen dem 6. und 8. Lebensjahr vornahmen (Modalität: Bildkarten statt Spielzeug; Itemanzahl: 24 statt 16), schienen keine Auswirkungen auf das Sortieren in der Lernphase zu haben, aber auf das Clustern in der Abrufphase und auf die Behaltensleistung.

Auch in der vorliegenden Untersuchung schien sich die Veränderung des Lernmaterials stärker auf das Verhalten in der Abrufphase als auf das in der Lernphase auszuwirken. Die zunehmende Stabilität des strategischen Verhaltens und der Erinnerungsleistung weist allerdings darauf hin, dass Kontexteinflüsse im Laufe der Grundschulzeit allgemein an Bedeutung verlieren.

\subsection{Bedeutung der Befunde vor dem Hintergrund der Strategie-Emergenz-Theorie}

Mit der Strategie-Emergenz-Theorie lieferte Hasselhorn (1996) einen wesentlichen Beitrag zur Gedächtnisentwicklung im Kindesalter. Wie er selber ausführt, besteht ihr Wert vor allem darin, dass sie nicht nur angibt ,in welchem Alter die Mehrzahl der Kinder die Fähigkeit und Bereitschaft zum strategischen Organisieren erwerben, sondern auch, dass eine beschleunigte Entwicklung des aufgabenspezifischen Metagedächtnisses die Ursache für den Erwerb dieser Fähigkeit darstellt. Anders als in der einschlägigen Literatur üblich, begnügt sich die SET auch nicht mit der Beschreibung von Defiziten oder Ineffizienzen der Strategienutzung, im frühen Stadium der entsprechenden metamemorialen Kompetenz. Vielmehr werden dezidierte Annahmen über die externen Bedingungen und die durch sie ausgelösten internen Faktoren solcher Nutzungsprobleme gemacht.“ (Hasselhorn, 1996; S. 180). Die vorliegende Untersuchung konnte die zentralen Aussagen der Strategie-Emergenz- 
Theorie bestätigen. Dazu gehört, dass die Fähigkeit und Bereitschaft zum strategischen Organisieren zwischen dem 8. und 10. Lebensjahr erworben wird, dass die beschleunigte Entwicklung des aufgabenspezifischen Metagedächtnisses eine wesentliche Ursache für den Erwerb der Fähigkeit zum strategischen Organisieren darstellt und dass die externen Bedingungen und die durch sie ausgelösten internen Faktoren von Nutzungsproblemen berücksichtigt werden müssen.

Gleichzeitig weist es aber auch auf Erklärungsgrenzen der Strategie-EmergenzTheorie hin und darauf, dass noch verschiedene Fragen offen sind (Hasselhorn, 1996; S. 180 ff.). Mit Hilfe der vorliegenden Untersuchungsbefunde soll nun im Folgenden versucht werden, Antworten auf einige dieser Fragen zu geben.

\section{Warum nutzen Zehnjährige ihr aufgabenspezifisches Metagedächtnis auch unter erschwerten Aufgabenbedingungen und andere nicht?}

Auf Grundlage der vorliegenden Befunde scheint es so zu sein, dass zu Beginn der vierten Klasse ein vorausgehendes niedriges Selbstkonzept (in der dritten Klasse) die Kinder davon abhält, die kategoriale Organisationsstrategie zu produzieren, auch wenn sie über das dafür erforderliche Strategiewissen verfügen. Eine zusätzliche Einflussgröße stellt das Ausmaß der Lernmotivation dar, das bei den Nichtstrategen deutlich geringer ausfällt.

Diese Kinder scheinen demnach zu glauben, dass die zur Disposition stehende Strategie anderen hilft, aber nicht ihnen. Obwohl objektiv gesehen ihre kognitive Leistungsfähigkeit genauso gut ist wie die der anderen Kinder, könnten sie subjektiv das Gefühl haben, dass der zusätzliche Kapazitätsaufwand für die Umsetzung des metamemorialen Wissens in entsprechende Kontrollprozesse sich nicht lohnt (vgl. Guttentag \& Ornstein, 1990).

Hinweise darauf, dass interindividuelle Differenzen in der metamemorialen Sensitivität eine Rolle spielen, gibt es in einem früheren Entwicklungsstadium. Am Ende der zweiten und am Ende der dritten Klasse ist das metakognitive Wissen der Nichtstrategen nicht so gut wie das der Strategen. Möglicherweise geben Nichtstrategen in diesem Entwicklungsstadium nur das Wissen von Erwachsenen wieder und nicht ihre eigenen Erkenntnisse (vgl. Reyna, 1989). Eine andere Möglichkeit besteht darin, dass ihnen der Transfer zwischen dem abstrakten Wissen und der konkreten Aufgabensituation nicht gelingt. Das metakognitive Wissen, über das sie verfügen, betrifft eventuell nur eine bestimmte Facette des Metagedächtnisses, die durch das gewählte Messinstrument abgetestet wird. 
Dieses Wissen könnte separat in abstrakter Form abgespeichert worden sein und damit keine Handlungsrelevanz für die Bearbeitung der aktuellen Gedächtnisanforderung besitzen.

\section{Welche kognitiven Mechanismen vermitteln das strategische Organisieren Zehnjähriger ohne angemessenes aufgabenspezifisches Metagedächtnis?}

Die aktuellen Befunde liefern Hinweise darauf, dass sich diese Kinder unmittelbar in der Entwicklung hin zum angemessenen Metagedächtnis befinden. Strategen, bei denen kein angemessenes Metagedächtnis festgestellt werden konnte, zeigten zum Zeitpunkt ihrer Strategieanwendung (Ende Klasse 2, Anfang Klasse 4) oder ein halbes Jahr zuvor (bei Strategen zu Beginn der dritten und am Ende der vierten Klasse), dass sie bereits über gute Strategiekenntnisse verfügten. Einige Kinder entdecken die Organisationsstrategie und ihre Nützlichkeit scheinbar erst während der Aufgabenbearbeitung oder kurz zuvor.

Die eingesetzte Methode erfasst möglicherweise auch nicht alle relevanten Aspekte des Metagedächtnisses. Die Kinder könnten aufgrund einer Überspezifizierung der Strategie oder aus motivationalen Gründen (geringes Selbstbewusstsein) das gewählte Vorgehen als nicht allgemein leistungsdienlich eingeschätzt haben.

\section{Welchen Entwicklungsveränderungen unterliegt die Sensitivität für die Nutzungsmöglichkeiten kategorialer Organisationsstrategien?}

Die metamemoriale Sensitivität determiniert nach Einschätzung von Hasselhorn (1996) den Zusammenhang zwischen der prinzipiellen Verfügbarkeit des metamemorialen Wissens über Organisationsstrategien und der aktuellen Nutzung dieses Wissens (Flavell \& Wellmann, 1977). Aufgrund der vorliegenden Befunde könnte es sich dabei sowohl um eine zusätzliche deklarative Wissenskomponente des Metagedächtnisses handeln als auch um eine Facette der Zentralen Exekutive. Ersteres lässt sich daraus ableiten, dass Kinder, die sich ohne angemessenes Metagedächtnis strategisch verhalten und daher sensitiv für die Nutzungsmöglichkeiten kategorialer Organisationsstrategien sein müssten, häufig gute Kenntnisse in Bezug auf spezielle Gedächtnisprozesse und Aufgabenanforderungen besitzen. Für den Zusammenhang mit einer zentral-exekutiven Kompontente spricht, dass diese Kinder bei der Aufgabenbearbeitung scheinbar über ein hohes Maß an freien kognitiven Ressourcen verfügen. Damit sich die Sensitivität für die Nutzungsmöglichkeiten kategorialer Organisationsstrategien in handlungswirksamer Weise äußern kann, scheint es wichtig zu 
sein, dass nicht die gesamte Kapazität von der Aufgabe absorbiert wird, sondern dass Ressourcen frei sind, um über das eigene Vorgehen $\mathrm{zu}$ reflektieren. Dieses würde durch verfügbare Kapazität der zentralen Exekutive gewährleistet, der damit eine entscheidende Bedeutung zukommt. Das Ausmaß an freien Ressourcen hängt sowohl von der eigenen kognitiven Leistungsfähigkeit als auch von kontextuellen Bedingungen ab, kann aber darüber hinaus auch von emotionalen oder motivationalen Zuständen der Person beeinflusst werden.

Die vorliegenden Befunde unterstützen diese Annahmen. In einer frühen Entwicklungsphase (am Ende der zweiten Klasse) und bei einer erhöhten Anzahl von zu lernenden Items scheint eine hohe Gedächtniskapazität die Kinder in die Lage zu versetzen, die Strategie auch ohne abstraktes Strategiewissen zu produzieren. Ein ausreichendes Maß an kognitiven Ressourcen scheint die Voraussetzung dafür zu schaffen, die vorhandene Sensitivität bei der Bewältigung der gestellten Anforderung zu nutzen.

\section{Was sind die Ursachen für die beschleunigte Entwicklung des aufgaben- spezifischen Metagedächtnisses über Organisationsstrategien zwischen 9 und 10} Jahren?

Als Ursachen für die beschleunigte Entwicklung des aufgabenspezifischen Metagedächtnisses über Organisationsstrategien zwischen 9 und 10 Jahren sind bisher sozioökonomische und erzieherische Einflüsse ebenso wie Kultureffekte diskutiert worden (vgl. Carr, Kurtz, Schneider, Turner \& Borkowski, 1989; Kurtz, Borkowski \& Deshmukh, 1988, Schneider, Borkowski, Kurtz \& Kerwin, 1986). Die vorliegenden Daten weisen aber auch auf einen am Ende der Grundschulzeit zunehmenden Zusammenhang zwischen der allgemeinen kognitiven Leistungsfähigkeit (Arbeitsgedächtniskapazität und Kurzzeitgedächtniskapazität) und dem Metagedächtnis hin. Während bereits am Ende der dritten Klasse das Metagedächtnis mit der ein halbes Jahr zuvor gemessenen Gedächtnisspanne korreliert ist, besteht dieser Zusammenhang in der vierten Klasse sowohl mit der früheren als auch mit der aktuellen Gedächtniskapazität. Dieses lässt die Vermutung $\mathrm{zu}$, dass am Ende der Grundschulzeit in das metakognitive Wissen sowohl die frühere als auch die aktuelle Leistungsfähigkeit einfließt, was auf eine zunehmend realistischere Einschätzung hindeutet. Eine gute kognitive Leistungsfähigkeit bietet gleichzeitig auch die Grundlage dafür, die vorhandenen metakognitiven Kenntnisse weiter zu verbessern.

In der vierten Klasse scheinen bei den meisten Kindern genügend Ressourcen zur Verfügung zu stehen, um über das eigene Lernverhalten zu reflektieren und ein angemessenes 
Metagedächtnis auszubilden. Zu Beginn der vierten Klasse sind Unterschiede im Metagedächtnis durch das frühere strategische Verhalten in der Lernphase erklärbar. Am Ende der vierten Klasse findet eine wechselseitige Beeinflussung statt, d.h. die Beziehung ist bidirektional. Eine differenzierte Betrachtung der individuellen Entwicklungsverläufe zeigt allerdings, dass es in jeder Altersstufe Kinder gibt, bei denen sich zuerst das strategische Verhalten und dann erst das Metagedächtnis verbessert, und Kinder, bei denen sich erst ein angemessenes Metagedächtnis herausbildet und dann das strategische Verhalten. Insgesamt weisen die aktuellen Befunde darauf hin, dass es sich bei der Entwicklung des Metagedächtnisses um ein komplexes Geschehen handelt, an dem verschiedene, sich wechselseitig beeinflussende Faktoren beteiligt sind.

Damit stellt sich die Frage, welche Bedeutung die aktuellen Befunde für das integrative Rahmenmodell der Informationsverarbeitung von Hasselhorn (1996) haben. Unter Berücksichtigung des Entwicklungsaspekts wird bereits deutlich, dass dieses Modell in seiner Grundstruktur mehrdimensional angelegt ist. Darüber hinaus widerspricht es nicht dem Grundgedanken von Hasselhorn (1996), neben der Beeinflussung durch Kontextfaktoren auch eine Beeinflussung durch motivationale Faktoren in Betracht zu ziehen, wie z.B. in dem metakognitiven Modell der „guten Informationsverarbeitung“ (Borkowski, 1996; Borkowski \& Thorpe, 1994), das in Kapitel 2.4.4 (S. 80 ff.) vorgestellt wurde.

Auf Grundlage der aktuellen Befunde könnte daher diskutiert werden, ob es sinnvoll ist, das integrative Rahmenmodell der Informationsverarbeitung von Hasselhorn (1996) zu erweitern. Beispielsweise scheinen die Zentrale Exekutive und das Metagedächtnis in vielen Fällen nicht voneinander abgrenzbar zu sein, sondern einen breiten Überschneidungsbereich $\mathrm{zu}$ besitzen, was unter anderem in der schwierigen Zuordnung des Sensitivität-Konstruktes deutlich wird. Neben kognitiven Faktoren sollte auch die Bedeutung des Selbstkonzepts und der Lernmotivation berücksichtigt werden, da sie nachweislich einen wichtigen Einfluss auf die Entwicklung effektiver Strategien haben.

Wesentlich ist außerdem, dass die einzelnen Komponenten in den verschiedenen Entwicklungsphasen eine unterschiedliche Relevanz für die Strategieproduktion und effektivität zu besitzen scheinen. Um die Dynamik des Entwicklungsgeschehens abzubilden, sollte daher versucht werden, auch die frühere kognitive Leistungsfähigkeit und emotionale Befindlichkeit mit einzubeziehen. Auch diese Erfahrungen beeinflussen die effektive Strategieentwicklung. 
Eine Modifikation des integrativen Rahmenmodells der Informationsverarbeitung von Hasselhorn (1996) auf der Grundlage der neu gewonnenen Befunde müsste folgendermaßen aussehen (vgl. Abbildung 6.1).

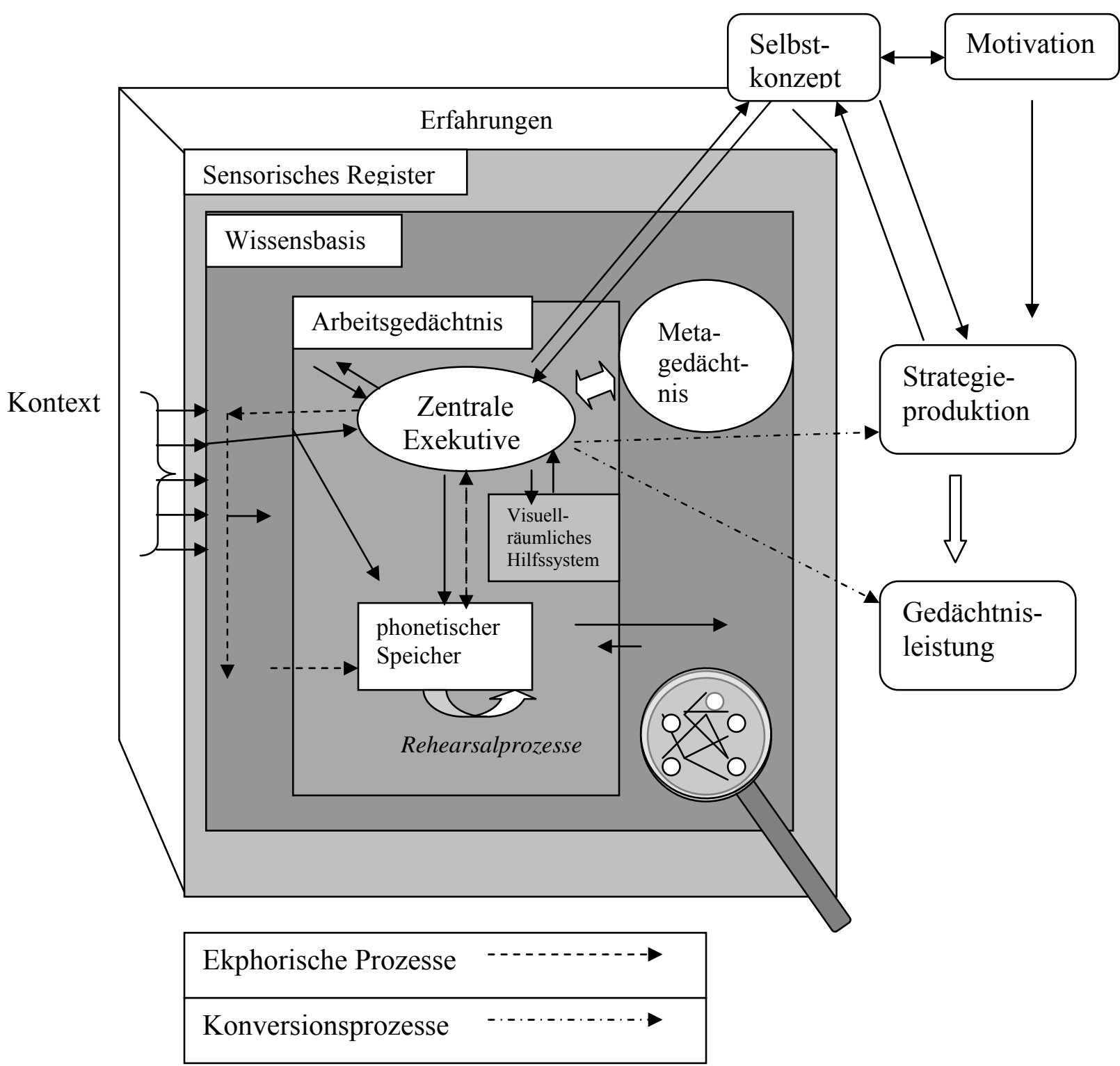

Abbildung 6.1: Erweitertes integratives Rahmenmodell der Informationsverarbeitung von Hasselhorn (1996)

Allerdings wird hier bereits deutlich, dass die Integration motivationaler Aspekte in das primär kognitiv ausgerichtete Modell nur unzureichend gelingt. Bevor ein umfassendes Modell für die effektive Strategieentwicklung aufgestellt werden kann, sind demnach noch weitere umfassende Untersuchungen notwendig. Die vorliegende Untersuchung stellt einen weiteren Schritt in diese Richtung dar. 


\subsection{Abschließende Bewertung}

Die Befunde der vorliegenden Untersuchung bestätigen, dass die effektive Entwicklung der kategorialen Organisationsstrategie in der zweiten Hälfte der Grundschulzeit stattfindet. Günstig für den weiteren Entwicklungsverlauf scheint dabei eine frühe Anwendung der Strategie zu sein, d.h. möglichst schon zu Beginn der dritten Klasse. Selbst wenn dieses Verhalten die Leistung nicht so verbessert wie erwartet, hat dieses keine negativen Auswirkungen, mittelfristig stellt sich bei fast allen Kindern der Nutzen ein. Wenn ein Nutzungsdefizit unterstellt wird, so muss stets berücksichtigt werden, auf welcher Grundlage die Effektivität des Verhaltens beurteilt wird. In der vorliegenden Untersuchung weisen die korrelativen Zusammenhänge zwischen dem kategorialen Organisiseren und der Behaltensleistung darauf hin, dass kategoriales Organisieren grundsätzlich mit einem Leistungsvorteil verbunden ist. Gleichzeitig zeigen sich aber beim Vergleich unterschiedlicher Altersstufen Hinweise auf ein Nutzungsdefizit. Dieses ist bei Zweitklässlern beim spontanen kategorialen Organisieren sowohl beim Lernen als auch beim Abruf von Informationen gegenüber Viertklässlern beobachtbar. Bei Verwendung von hoch-typischem Material weisen Zweitklässler gegenüber Viertklässlern ein Nutzungsdefizit beim kategorialen Abrufen von Informationen auf, ebenso wie Grundschüler gegenüber Sechstklässlern beim kategorialen Lernen von Informationen. Unter Verwendung einer höheren Anzahl von Items erweisen sich Viertklässler gegenüber Sechstklässlern beim kategorialen Lernen von Informationen als nutzungsdefizitär. Mit zunehmendem Alter ist also noch eine weitere Steigerung der Strategieeffektivität möglich. Dieses hängt allerdings mit der allgemein besseren Leistungsfähigkeit älterer Kinder zusammen und sagt nichts darüber aus, ob jüngere Kinder ebenfalls von strategischem Verhalten profitieren. Auf Individualebene kann zu den einzelnen Messzeitpunkten bei ungefähr einem Drittel der Kinder eine geringere Leistungssteigerung beobachtet werden als erwartet. Auch die erste Strategieproduktion erweist sich nicht bei allen Kindern als nützlich. Zu Beginn der dritten und vierten Klasse bleibt die Leistungssteigerung bei einem Drittel der Kinder, am Ende der dritten und vierten Klasse bei zwei Drittel der Kinder hinter den Erwartungen zurück. Dabei muss allerdings berücksichtigt werden, dass die Erwartungen bezüglich einer Leistungssteigerung sehr hoch gesteckt wurden. $\mathrm{Zu}$ diskutieren bleibt, ob nicht auch eine eher geringe Verbesserung der Behaltensleistung als nützlich bezeichnet werden sollte. Tut man dies, profitieren fast alle Kinder von ihrer Strategieproduktion und das Phänomen des Nutzungsdefizits wäre kaum beobachtbar. 
Als entscheidende Determinante für das kategoriale Organisieren kristallisiert sich in der vorliegenden Untersuchung das Metagedächtnis heraus, das in der vierten Klasse als angemessen bezeichnet werden kann. Wie die vorliegenden Befunde zeigen, kann aber auch ohne ein angemessenes Metagedächtnis die kategoriale Organisationsstrategie angewendet werden. Eine gute Vorausssetzung dafür bietet eine überdurchschnittliche Gedächtniskapazität, die dafür sorgt, dass in ausreichendem Maß freie Ressourcen zur Verfügung stehen.

Am Ende der dritten Klasse erweisen sich ein angemessenes Metagedächtnis und eine überdurchschnittliche Arbeitsgedächtniskapazität zusammen als hinreichende Bedingung, um die kategoriale Organisationsstrategie spontan effektiv zu produzieren.

Darüber hinaus scheint aber auch entscheidend $\mathrm{zu}$ sein, wie hoch die eigene Leistungsfähigkeit bei der ersten Auseinandersetzung mit der Aufgabenanforderung eingeschätzt wird.

Ein hohes Selbstkonzept in der zweiten Klasse scheint einen positiven Einfluss auf die effektive Strategieproduktion am Ende der dritten und zu Beginn der vierten Klasse zu haben. Ein niedrigeres Selbstkonzept und eine geringe Lernmotivation zu Beginn der vierten Klasse können wiederum dazu führen, dass eine Strategie von den Kindern nicht mehr eingesetzt wird.

Für die Produktion der kategorialen Organisationsstrategie unter Verwendung einer höheren Itemanzahl sind am Ende der dritten Klasse ein angemessenes Metagedächtnis und eine überdurchschnittliche Lernmotivation hinreichend. Wird die Strategie am Ende der dritten Klasse trotz vorhandenem Strategiewissen nicht eingesetzt, so lässt sich dieses auf die unzureichende Motivation zurückführen.

Wenn eine Strategie zu Beginn der vierten Klasse ineffektiv produziert wird, obwohl ein angemessenes Metagedächtnis vorhanden ist, so kann eine Ursache dafür sein, dass die Kinder noch nicht so lange über dieses Strategiewissen verfügen. Als Beleg dafür kann das schlechtere metakognitive Wissen der ineffektiven Strategen ein halbes Jahr vor ihrer Strategieproduktion gesehen werden. Bei einer erhöhten Itemanzahl kommt darüber hinaus als weiterer Einflussfaktor die geringere Gedächtniskapazität in Betracht. Hinweise darauf liefert die Beobachtung, dass unter den Kindern mit angemessenem Metagedächtnis die ineffektiven Strategen am Ende der dritten Klasse zuvor eine geringere Arbeitsgedächtniskapazität und die ineffektiven Strategen zu Beginn der vierten Klasse sowohl eine geringere Arbeits- als auch eine geringere Kurzzeitgedächtniskapazität besaßen. 
Ein angemessenes Metagedächtnis kann nicht garantieren, dass eine Strategie produziert oder sogar effektiv eingesetzt wird. Umgekehrt kann aus der Tatsache, dass Kinder in der Versuchssituation kein angemessenes Metagedächtnis erkennen lassen, nicht geschlossen werden, dass sie deswegen die relevante Strategie nicht einsetzen. Hier zeigt sich die Vielschichtigkeit des Metagedächtnisses, auf die bereits im Vorfeld hingewiesen wurde. Um in einer konkreten Situation eine bestimmte Strategie einzusetzen, scheint es nicht unbedingt erforderlich zu sein, dass abstrakte Kenntnisse über Gedächtnis- und Lernprozesse vorhanden sind. Die Beobachtung, dass Kinder, die ohne angemessenes Metagedächtnis eine Strategie produzieren, über bessere Strategiekenntnisse verfügen als Nichtstrategen, zeigt, dass sie sich aber auf dem Weg zu einem guten Metagedächtnis befinden. Dieser Prozess ist aber mit einer Ansammlung von Erfahrungen und ihrer persönlichen Bewertung verbunden. Dabei kann es durchaus vorkommen, dass ein Kind sicher ist, dass das von ihm gewählte Vorgehen am besten ist, ohne von dem eigenen Vorteil auf einen allgemeinen Vorteil zu schließen.

Umgekehrt kann ein Kind durchaus der Meinung sein, dass die Anwendung einer Strategie im Allgemeinen vorteilhaft bei der Bearbeitung der Aufgabe ist, aber diesen Nutzen für die eigene Person anzweifeln. Darüber hinaus sind auch viele andere Gründe denkbar, warum ein Verhalten trotz besseren Wissens nicht gezeigt wird. Um nur einen zu nennen, kann der Aufwand für eine Strategie gescheut werden oder die Wahl einer schwierigeren Lösungsweges als neue Herausforderung angesehen werden.

Die Bedeutung des Metagedächtnisses muss demnach stets im Zusammenhang mit anderen internen Faktoren (kognitiven und motivationalen) und den kontextuellen Rahmenbedingungen gesehen werden.

Trotz dieser Einschränkungen kommt dem Metagedächtnis allerdings zu jeder Zeit eine herausragende Bedeutung für die Produktion und Effektivität der kategorialen Organisationsstrategie zu. Dieses lässt sich darauf zurückführen, dass sich gute Strategiekenntnisse positiv auf die kognitive und motivationale Entwicklung der Kinder auswirken. Da dieses Vorgehen grundsätzlich geeignet für die Bearbeitung der gewählten „Sort-Recall“-Anforderung ist, erfahren die Kinder langfristig auch einen Nutzen. Dieses wirkt sich nicht nur positiv auf das Selbstkonzept und die zukünftige Lernmotivation aus, sondern schafft über den zunehmenden Automatisiertheitsgrad auch neue Ressourcen, die für andere Aktivitäten zur Verfügung stehen. 
Für diesen positiven Entwicklungsprozess scheint es allerdings wichtig zu sein, dass Kinder bereits während der Grundschulzeit Erfahrungen im Umgang mit Strategien sammeln. Wie die vorliegende Untersuchung zeigt, hat ein vorübergehendes Nutzungsdefizit in der Mitte der Grundschulzeit keine negativen Auswirkungen auf die Strategieentwicklung. Die Kinder behalten trotzdem die Strategie bei und profitieren später davon. Dieser Überoptimismus wird in der vierten Klasse von einer realistischen Einschätzung der eigenen Leistungsmöglichkeiten abgelöst, was sich in der vorliegenden Untersuchung in der Verbesserung des Metagedächtnisses und des Selbstkonzepts wiederspiegelt. Wenn Kinder am Ende der vierten Klasse nicht von einem bestimmten Verhalten profitieren, so ziehen sie vermehrt daraus die Konsequenz, dieses Verhalten nicht mehr zu zeigen. Bezogen auf die Anwendung von Strategien entgeht ihnen allerdings dadurch die Möglichkeit, die strategischen Kompetenzen zu verbessern. Wie die vorliegenden Befunde zeigen, ist für eine erstmalige Strategieanwendung am Ende der Grundschulzeit eine erhöhte Motivation erforderlich.

Eine frühere Erprobung der Strategie scheint mit wesentlich weniger Aufwand und Belastung verbunden zu sein. Aus diesem Grund sollte bereits frühzeitig damit begonnen werden, Kindern Aufgaben $\mathrm{zu}$ stellen, in denen sie die Anwendung von Strategien ausprobieren können. Besonders das kategoriale Organisieren stellt eine Strategie dar, von der Kinder in ihrem weiteren Entwicklungsverlauf profitieren können. Sie hilft dabei, die eigene Umwelt zu strukturieren und dadurch den Überblick zu behalten. Diese Fähigkeit ist nicht nur für Kinder wichtig, sondern bis ins hohe Erwachsenenalter relevant. Erinnern wir uns an die Einkaufssituation in der Einleitung dieser Arbeit, so darf man optimistisch sein, dass auch das Kind bald diese Erfahrung macht. 


\section{Zusammenfassung}

Im Bereich der kognitiven Entwicklungspsychologie nehmen Strategien einen hohen Stellenwert ein. Ihre Genese wird aufgrund zahlreicher Querschnittstudien oft als kontinuierlicher Prozess beschrieben, bei dem strategisches Verhalten zuerst nicht (Mediationsdefizit), dann unter Anleitung (Produktionsdefizit) und später spontan produziert wird. Führt dieser selbständige Strategiegebrauch zu keinem nennenswerten Leistungsvorteil, so wird von einem Nutzungsdefizit gesprochen. Aktuelle längsschnittliche (Sodian \& Schneider, 1999) und mikrogenetische Studien (Schlagmüller \& Schneider, 2002) ließen Zweifel an der Allgemeingültigkeit dieser Annahmen aufkommen, da ihre Befunde eher für einen sprunghaften Entwicklungsverlauf sowie eine ausreichende Strategieeffektivität bei älteren Grundschulkindern sprachen. Zur Erklärung unterschiedlicher strategischer Kompetenzen wurde neben basalen kognitiven Prozessen (z.B. Informationsverarbeitungsgeschwindigkeit) vor allem das umfangreichere metakognitive und bereichsspezifische Wissen herangezogen. Unter Verweis auf die enge Verzahnung motivationaler und kognitiver Prozesse scheint darüber hinaus die Berücksichtigung von Lernmotivation und leistungsbezogenen Facetten des Selbstkonzepts angebracht zu sein.

Die vorliegende Untersuchung liefert weitere Informationen über die intraindividuellen Entwicklungsveränderungen und interindividuellen Unterschiede beim kategorialen Organisieren und diskutiert die Gründe für diese Differenzen. Die Befunde bestätigen, dass kategoriales Organisieren bei der Bearbeitung einer „Sort-Recall“Anforderung wesentlich dazu beiträgt, gute Reproduktionsleistungen zu erbringen. Aber auch wenn die meisten Kinder von ihrem Strategieeinsatz profitieren, so finden sich dennoch bei einigen Hinweise auf ein Nutzungsdefizit. Eine entscheidende Rolle bei der Entwicklung der effektiven Organisationsstrategie spielt erwartungsgemäß das aufgabenspezifische Metagedächtnis. Darüber hinaus zeigt sich aber, dass ein angemessener Strategiegebrauch auch ohne elaboriertes Strategiewissen möglich ist, wenn eine hohe Arbeitsgedächtniskapazität oder Ansätze metakognitiver Kenntnisse vorhanden sind. Frühes kategoriales Organisieren scheint sich positiv auf die Entwicklung strategischer Kompetenzen auszuwirken. Am Ende der vierten Klasse setzen aus motivationalen Gründen zunehmend mehr Kinder die Strategie nicht mehr ein. Insgesamt weisen die Befunde darauf hin, dass Aussagen über individuelle strategische Kompetenzen stets unter Berücksichtigung der kognitiven und motivationalen Voraussetzungen sowie der vorliegenden Rahmenbedingungen zu treffen sind. 


\section{Literaturverzeichnis}

Ackerman, B. P. (1982). Retrieval variability: The inefficient use of retrieval cues by young children. Journal of Experimental Child Psychology, 33, 413-428.

Ackerman, B. P. (1983). Encoding distinctiveness and the encoding shift penalty in children and adults. Journal of Experimental Child Psychology, 36, 257-283.

Ackerman, B. P. (1984). Item-specific and relational encoding effects in children's recall and recognition memory for words. Journal of Experimental Child Psychology, 37, 426450.

Ackerman, B. P. (1985a). Children's retrieval deficits. In C. J. B. M. Pressley (Ed.), Basic processes in memory development (pp. 1-46). New York: Springer-Verlag.

Ackerman, B. P. (1985b). Children's use of context and and category cues to retrieve episodic information from memory. Journal of Experimental Child Psychology, 40, 420-438.

Ackerman, B. P. (1985c). Children's use of retrieval cues to "describe" episodic information in memory: Problems of constructability and discriminability. Journal of Experimental Child Psychology., 40, 193-217.

Ackerman, B. P. (1985d). Constraints on retrieval search for episodic information in children and adults. Journal of Experimental Child Psychology, 40, 152-180.

Ackerman, B. P. (1985e). The effects of specific and categorical orienting on children's incidental and intentional memory for pictures and words. Journal of Experimental Child Psychology, 39, 300-325.

Ackerman, B. P. (1987). Descriptions: A model of nonstrategic memory development. In H. W. Reese (Ed.), Advances in child development and behavior. (Vol. 20, pp. 143-183). Orlando, Florida: Academic Press.

Ackerman, B. P. (1996). Induction of a memory retrieval strategy by young children. Journal of Experimental Child Psychology, 62, 243-271.

Ackerman, B. P., \& Hess, L. (1982). The effects of encoding distinctiveness on retrieval variability in children and adults. Journal of Experimental Child Psychology, 33, 465474.

Ackerman, B. P., \& Rathburn, J. (1984). Developmental differences in the use of retrieval cues to describe episodic information in memory. Journal of Experimental Child Psychology, 38, 147-173. 
Ackerman, B. P., \& Rust-Kahl, E. (1982). The effects of contrastive encoding of semantic information on children's memory for words. Journal of Experimental Child Psychology, 34, 414-474.

Andreassen, C., \& Waters, H. S. (1989). Organisation during study: Relationships between metamemory, strategy use, and performance. Journal of Educational Psychology, 81(2), 190-195.

Arlin, M., \& Brody, R. (1976). Effects of spatial presentation and blocking on organization and verbal recall at three grade levels. Developmental Psychology, 12, 113-118.

Atkinson, J. W. (1982). Old and new conceptions of how expected consequences influence actions. In N. Fether (Ed.), Expectations and actions: Expectancy value models in psychology. Hillsdale, NJ: Erlbaum.

Atkinson, R. C., \& Shiffrin, R. M. (1968). Human memory: A proposed system and its control processes. In K. W. Spence \& J. T. Spence (Eds.), The psychology of learning and motivation. (Vol. 2, pp. 89-195). New York: Academic Press.

Baddeley, A. D. (1986). Working memory. Oxford: Oxford University Press.

Baddeley, A. D. (2000). Short-term and working memory. In E. Tulving \& F. J. M. Craik (Eds.), The Oxford Handbook of Memory (pp. 77-92). Oxford: University Press.

Baddeley, A.D. (2002). Is working memory still working? European Psychologist, 7(2), 8597.

Baddeley, A. D., \& Hitch, G. J. (1974). Working memory. In G. H. Bower (Ed.), The psychology of learning and motivation (Vol. 8, pp. 47-87). New York: Academic Press.

Bandura, A. (1989). Regulation of cognitive processes through perceived self-efficacy. Developmental Psychology, 25(5), 729-735.

Beal, C. R. (1995). Development of knowledge about the use of cues to aid prospective retrieval. Child Development, 56, 631-642.

Belmont, J. M., \& Borkowski, J. G. (1988). A group-administered test of children's metamemory. Bulletin of the Psychonomic Society, 26(3), 206-208.

Best, D. L. (1993). Inducing children to generate mnemonic organizational strategies: An examination of long-term retention and materials. Developmental Psychology, 29, 324-336.

Beuhring, T., \& Kee, D. W. (1987a). Developmental relationships among metamemory, elaborative strategy use, and associative memory. Journal of Experimental Child Psychology, 44, 377-400. 
Beuhring, T., \& Kee, D. W. (1987b). Elaboration and associative memory development: The metamemory link. In M. A. M. M. Pressley (Ed.), Imagery and related mnemonic processes:Theories and applications (pp. 257-273). New York: Springer-Verlag.

Bevan, W., \& Steger, J. A. (1971). Free recall and abstractness of stimuli. Science, 172, $597-$ 599.

Bisanz, G. L., Versonder, G. T., \& Voss, J. F. (1978). Knowledge of one's own responding and the relation of such knowledge to learning. Journal of Experimental Child Psychology, 25, 116-128.

Bjorklund, D. F. (1980a). Children's identification of category relations in lists presented for recall. Journal of Genetic Psychology, 136, 45-53.

Bjorklund, D. F. (1980b). Developmental differences in the timing of childrens awareness of category relations in free recall. International Journal of Behavioral Development, 3, 61-70.

Bjorklund, D. F. (1985). The role of conceptual knowledge in the development of organization in children's memory. In C. J. B. M. Pressley (Ed.), Basic processes in memory development: Progress in cognitive development research. (pp. 103-142). New York: Springer.

Bjorklund, D. F. (1987). How age changes in knowledge base contribute to the development of children's memory: An interpretative review. Developmental Review, 7, 93-130.

Bjorklund, D. F. (1988). Acquiring a Mnemonic: Age and Category Knowledge Effects. Journal of Experimental Child Psychology, 45, 71-87.

Bjorklund, D. F. (1990). Children's strategies: Contemporary views of cognitive development. Hillsdale, NJ: Erlbaum.

Bjorklund, D.F. (2000). Children's thinking: Developmental function and individual differences ( 3 ed.). Belmont, CA: Wadsworth.

Bjorklund, D.F., \& Bjorklund, B.R. (1985). Organization versus item effects of an elaborated knowledge base on children's memory. Developmental Psychology, 21, 1120-1131.

Bjorklund, D. F., \& Buchanan, J. J. (1989). Developmental and knowledge base differences in the acquisition and extension of a memory strategy. Journal of Experimental Child Psychology, 48, 451-471.

Bjorklund, D. F., \& Coyle, T. R. (1995). Utilization deficiencies in the development of memory strategies. In F. E. Weinert \& W. Schneider (Eds.), Memory Performance and Competencies: Issues in Growth and Development (pp. 161-179). Hillsdale, N. J.: Lawrence Erlbaum. 
Bjorklund, D. F., Coyle, T. R., \& Gaultney, J. F. (1992). Developmental differences in the acquisition and maintenance of an organizational strategy: Evidence for the utilization deficiency hypothesis. Journal of Experimental Child Psychology, 54, 434-448.

Bjorklund, D. F., \& Green, B. L. (1992). The adaptive nature of cognitive immaturity. American Psychologist, 47, 46-54.

Bjorklund, D. F., \& Harnishfeger, K. K. (1987). Developmental differences in the mental effort requirements for the use of an organizational strategy in free recall. Journal of Experimental Child Psychology, 44, 109-125.

Bjorklund, D. F., \& Harnishfeger, K. K. (1990). Children's strategies: Their definition and origins. In D.F. Bjorklund (Ed.), Children's strategies: Contemporary views of cognitive development. (pp. 309-323). Hillsdale, NJ: Erlbaum.

Bjorklund, D. F., \& Jacobs, J. W. (1985). Associative and categorical processes in children's memory: The role of automaticity in the development of organization in free recall. Journal of Experimental Child Psychology, 39, 599-617.

Bjorklund, D. F., \& Miller, P. H. (1997). New themes in strategy development. Developmental Review, 17, 407-410.

Bjorklund, D. F., Miller, P. H., Coyle, T. R., \& Slawinski, J. L. (1997). Instructing children to use memory strategies: Evidence of utilization deficiencies in memory training studies. Developmental Review, 17, 411-441.

Bjorklund, D. F., Muir-Broaddus, J. E., \& Schneider, W. (1990). The role of knowledge in the development of strategies. In D.F. Bjorklund (Ed.), Children's Strategies: Contemporary Views of cognitive Development (pp. 93-128). Hillsday, N. J.: Lawrence Erlbaum.

Bjorklund, D. F., \& Schneider, W. (1996). The interaction of knowledge, aptitudes, and strategies in children's memory performance. In H. W. Reese (Ed.), Advances in Child Development and Behavior (Vol. 25, pp. 59-89). San Diego: Academic Press.

Bjorklund, D. F., Schneider, W., Cassel, W. S., \& Ashley, E. (1994). Training and extension of a memory strategy: Evidence for utilization deficiencies in the acquisition of an organizational strategy in high- and low-IQ children. Child Development, 65(3), 951965.

Bjorklund, D. F., Thompson, B. E., \& Ornstein, P. A. (1983). Developmental trends in children's typicality judgements. Behavior Research Methods \& Instrumentation, 15, $350-356$. 
Bjorklund, D. F., \& Zeman, B. R. (1982). Children's organization and metamemory awareness in their recall of familiar information. Child Development, 53, 799-810.

Black, M. M., \& Rollins, H. A. (1982). The effects of instrucional variables on young children's organization and free recall. Journal of Experimental Child Psychology, 33(1), 1-19.

Blair, R., Perlmutter, M., \& Myers, N. A. (1978). Effects of unlabeled and labeled picture cues on very young children's memory for location. Bulletin of the Psychonomic Society, 11, 46-48.

Blöte, A.W., Resing, W.C., Mazer, P., \& Van Noort, D. A. (1999). Young children's organizational strategies on a same-different task: A microgenetic study and a training study. Journal of Experimental Child Psychology, 74, 21-43.

Bong, M., \& Clark, R.E. (1999). Comparison between self-concept and self-efficacy in academic motivation research. Educational Psychologist, 34(3), 139-153.

Borkowski, J. C. (1996). Metacognition: Theory or chapter heading? Learning \& Individual Differences, 8(4), 391-402.

Borkowski, J. G., Carr, M., Rellinger, E., \& Pressley, M. (1990). Self-regulated cognition: Interdependence of metacognition, attributions, and self esteem. In B. B. L. Idol (Ed.), Dimensions of thinking and cognitive instruction (Vol. 1, pp. 53-92). Hillsdale, NJ: Erlbaum.

Borkowski, J. G., Johnston, N. B., \& Reid, N. K. (1987). Metacognition, motivation, and the transfer of control processes. In S. J. Ceci (Ed.), Handbook of cognitive, social, and neuropsychological aspects of learning disabilities. (pp. 147-173). Hillsdale, NJ: Erlbaum.

Borkowski, J. G., \& Krause, A. J. (1985). Metacognition and attributional beliefs. In G. d'Ydewalle (Ed.), Cognition, information processing, and motivation. (pp. 557-567). Amsterdam: Elsevier.

Borkowski, J. G., Milsted, M., \& Hale, C. (1988). Components of children's metamemory: Implications for strategy generalization. In F. E. W. M. Perlmutter (Ed.), Memory development: Universal changes and individual differences. (pp. 73-100). Hillsdale, NJ: Erlbaum.

Borkowski, J. G., \& Muthukrishna, N. (1995). Learning environments and skill generalization: How context faciliate regulatory processes and efficacy beliefs. In F. E. W. W. Schneider (Ed.), Memory performance and competencies: Issues of growth and development. (Vol. 1995). Mahwah, NJ: Erlbaum. 
Borkowski, J. C., \& Thorpe, P. K. (1994). Self-regulation and motivation: A life-span perspective on underachievement. In D. H. Schunk \& B. J. Zimmerman (Ed.), Selfregulation of learning and performance. Issues and educational applications. (pp. 2544). Hillsdale, NJ: Erlbaum.

Borkowski, J. G., \& Turner, L. A. (1990). Transsituational characteristics of metacognition. In F. E. Weinert (Ed.), Interactions among aptitudes, strategies, and knowledge in cognitive performance. (pp. 159-176). New York: Springer.

Borkowski, J. G., Weyhing, R. S., \& Carr, M. (1988). Effects of attributional retraining on strategy-based reading comprehension in learning-disabled students. Journal of Educational Psychology, 80(1), 46-53.

Bousfield, A. K., \& Bousfield, W. A. (1966). Measurement of clustering and of sequential constanties in repeated free recall. Psychological Reports, 19(3), 935-942.

Bousfield, W. A. (1953). The occurence of clustering in the recall of randomly arranged associates. Journal of Genetic Psychology, 49, 229-240.

Bousfield, W. A., Esterson, J., \& Whitmarsh, G. A. (1958). A study of developmental changes in conceptual and perceptual associative clustering. Journal of Genetic Psychology, 92, 95-102.

Bower, G. H. (2000). A brief history of memory research. In E. Tulving \& F. J. M. Craik (Eds.), The Oxford Handbook of Memory (pp. 3-32). Oxford: University Press.

Brainerd, C. J. (1982). Children's concept learning as rule-sampling systems with Markovian properties. In C. J. Brainerd (Ed.), Children's logical and mathematical cognition: Progress in cognitive development research. (pp. 177-212). New York: SpringerVerlag.

Brainerd, C. J. (1985). Basic processes in memory development: Progress in cognitive development research., Springer series in cognitive development. New York: Springer.

Brainerd, C. J. (1995). Interference Processes in Memory Development: The Case of Cognitive Triage. In Interference and Inhibition of Cognition (pp. 105-139).

Brainerd, C. J., \& Howe, M. L. (1982). Stages-of-learning analysis of developmental interactions in memory, with illustrations from developmental interactions in picturewords effects. Developmental Review, 2, 251-273.

Brainerd, C. J., Howe, M. L., \& Kingma, J. (1982). An identifiable model of two stage learning. Journal of Mathematical Psychology, 26, 263-293. 
Brainerd, C. J., Howe, M. L., Kingma, J., \& Brainerd, S. H. (1984). On the measurement of storage and retrieval contributions to memory development. Journal of Experimental Child Psychology, 37, 478-499.

Breckler, S. J., \& Greenwald, A. G. (1986). Motivational facets of the self. In R. M. Sorrentino \& E. T. Higgins (Eds.), Handbook of motivation and cognition: Foundations of social behavior. (pp. 145-164).

Brown, A. L. (1978). Knowing when, where, and how to remember: A problem of metacognition. In R. Glaser (Ed.), Advances in instructional psychology (pp. 77-165). Hillsdale, N.J.: Erlbaum.

Brown, A. L., Bransford, J. D., Ferrara, R. A. \& Campione, J. C. (1983). Learning, remembering, and understanding. In J. H. F. E. M. Markman (Ed.), Handbook of child psychology (4 ed., Vol. 3, pp. S. 77-166). New York: Wiley.

Brown, A. L., \& DeLoache, J. S. (1978). Skills, plans, and self-regulation. In R.S. Siegler (Ed.), Children's thinking: What develops? (pp. 3-36). Hillsdale, NJ: Lawrence Erlbaum Associates.

Brown, G. D. A., \& Hulme, C. (1995). Modeling item length effects in memory span: No rehearsal needed? Journal of Memory and Language, 34(5), 594-621.

Brown, R., \& Berko, J. (1960). Word association and the acquisition of grammar. Child Development, 31, 1-14.

Bryan, J., \& Luszcz, M. A. (1996). Speed of information processing as a mediator between age and free-recall performance. Psychology and Aging, 11(1), 3-9.

Carr, M., Kurtz, B. E., Schneider, W., Turner, L. A., \& Borkowski, J. G. (1989). Strategy acquisition and transfer: Environmantal influences on metacognitive development. Developmental Psychology, 25, 765-771.

Case, R., Kurland, D. M., \& Goldberg, J. (1982). Operational efficiency and the growth of short-term memory span. Journal of Experimental Child Psychology, 33, 386-404.

Cavanaugh, J. C. (1989). The importance of awareness in memory aging. In D. C. R. B. A. W. L.W. Poon (Ed.), Everyday cognition in adulthood and late life (pp. 416-436). Cambridge: Cambridge University Press.

Cavanaugh, J. C., \& Perlmutter, M. (1982). Metamemory: A critical examination. Child Development, 53(1), 11-28.

Ceci, S. J. (1980). A developmental study of multiple encoding and its relationship to agerelated changes in free recall. Child Development, 51, 892-895. 
Ceci, S. J., \& Howe, M. J. A. (1978a). Semantic knowledge as a determinant of developmental differences in recall. Journal of Experimental Child Psychology, 26, 230-245.

Ceci, S. J., \& Howe, M. J. A. (1978b). Age-related differences in free recall as a function of retrieval flexibility. Journal of Experimental Child Psychology, 26, 432-442.

Ceci, S. J., Lea, S. E. G., \& Howe, M. J. A. (1980). Structural analysis of memory traces in children from 4 to 10 years of age. Developmental Psychology, 16, 203-212.

Chechile, R. A., \& Meyer, D. L. (1976). A Bayesian procedure for separately estimating storage an retrieval components of forgetting. Journal of Mathematical Psychology, 13, 269-295.

Chechile, R. A., \& Richman, C. L. (1982). The interaction of semantic memory with storage and retrieval processes. Developmental Review, 2(3), 237-250.

Chechile, R. A., Richman, C. L., Topinka, C., \& Ehrensbeck, K. (1981). A developmental study of the storage and retrieval of information. Child Development., 52, 251-259.

Chi, M. T. H. (1978). Knowledge structure and memory development. In R. S. Siegler (Ed.), Children's thinking: What develops? (pp. 73-96). Hillsdale, NJ: Erlbaum.

Chi, M.T.H. (1981). Knowledge development and memory performance. In J. P. D. N. O. C. M.P. Friedman (Ed.), Intelligence and learning (pp. 221-229). New York: Plenum.

Chi, M. T. H. (1985). Interactive roles of knowledge and strategies in the development of organized sorting and recall. In S. Chipman, J. Segal \& R. Glaser (Eds.), Thinking and learning skills: Current research and open questions. (Vol. 2, pp. 457-483). Hillsdale, NJ: Erlbaum.

Chi, M. T. H., \& Ceci, S. J. (1987). Content knowledge: Its role, representation, and restructuring in memory development. In H. W. Reese (Ed.), Advances in child development and behavior (Vol. 19, pp. 29-39). Orlando, FL: Academic Press.

Chi, M. T. H., Hutchinson, J. E., \& Robin, A. F. (1989). How inferences about novel domainrelated concepts can be constrained by structured knowledge. Quarterly, 35, 27-62.

Chi, M. T. H., \& Koeske, R. D. (1983). Network representation of a child's dinosaur knowledge. Developmental Review, 19, 29-39.

Chin-Parker, S. R., Brian H. (2002). The effect of category learning on sensitivity to withincategory correlations. Memory \& Cognition, 30(3), 353-362.

Cho, S., \& Ahn, D. (2003). Strategy acquisition and maintenance of gifted and nongifted young children. Council for exceptional children., 69(4), 497-505. 
Cole, M., Frankel, F., \& Sharp, D. (1971). Development of free recall in children. Developmental Psychology, 4, 109-123.

Collins, A. M., \& Loftus, E. F. (1975). A spreading-activation theory of semantic processing. Psychological Review, 82, 407-428.

Cornoldi, C. (1998). The impact of metacognitive reflection on cognitive control. In G. N. Mazzoni \& T. O. Nelson (Eds.), Metacognition and cognitive Neuropsychology: Monitoring and control Processes (pp. 139-159). Mahwah, N. J.: Lawrence Erlbaum.

Corsale, K., \& Ornstein, P. A. (1980). Developmental changes in children's use of semantic information in recall. Journal of Experimental Child Psychology, 30, 231-245.

Cox, B. D., Ornstein, P. A., Naus, M.J., Maxfield, D., \& Zimler, J. (1989). Children's concurrent use of rehearsal and organizational strategies. Developmental Psychology, 25(4), 619-627.

Cox, D., \& Waters, H. S. (1986). Sex differences in the use of organization strategies: A developmental analysis. Journal of Experimental Child Psychology, 41(1), 18-37.

Coyle, T. R., \& Bjorklund, D. F. (1996). The development of strategic memory: A modified microgenetic assessment of utilization defidiencies. Cognitive Development, 11(2), 295-314.

Coyle, T. R., \& Bjorklund, D. F. (1997). Age differences in, and consequences of, multipleand variable-strategy use on a multitrial sort-recall task. Developmental Psychology, 33(2), 372-380.

Cultice, J.C., Somerville, S. C., \& Wellman, H. M. (1983). Preschooler's memory monitoring: Feeling-of-knowing judgements. Child Development, 54, 1480-1486.

Cunningham, J. G., \& Weaver, S. L. (1989). Young children's knowledge of their memory span: Effects of task and experience. Journal of Experimental Child Psychology, 48, 32-44.

Davis, A. J. (1976). Categorization styles in older children and adolescents. Journal of Youth and Adolescens, 5, 187-200.

de la Mata, M. L., \& Sanchez, J. A. (1991). Educacion formal y acciones de agrupacion y memoria en adultos: Un estudio microgenetico. Infancia y Aprendizaje, 53(Special Issue: Spanish historica-cultural psychology: Language and development.), 75-97.

DeLoache, J. S., \& Brown, A. L. (1984). Where do i go next? Intelligent searching by very young children. Developmental Psychology, 20(1), 37-44.

DeLoache, J. S., Cassidy, S. J., \& Brown, A. L. (1985). Precursors of mnemonic strategies in very young children's memory. Child Development, 56, 125-137. 
DeMarie-Dreblow, D., \& Miller, P. H. (1988). The development of children's strategies for selective attention: Evidence for a transitional period. Child Development, 59, 1541513.

Dempster, F. N. (1981). Memory Span: Sources of individual and developmental differences. Psychological Bulletin, 89, 64-100.

Dempster, F. N. (1985). Short-term memory development in childhood and adolescence. In C. J. B. M. Pressley (Ed.), Basic processes in memory development. Progress in cognitive development research. (pp. 209-248). New York: Springer.

Dufresne, A., \& Kobasigawa, A. (1989). Children's spontaneou allocation of study time: Differential and sufficient aspects. Journal of Experimental Child Psychology, 47, 274-296.

Emmerich, H. J., \& Ackerman, B. P. (1978). Developmental Differences in Recall: Encoding or Retrival? Journal of Experimental Child Psychology, 25, 514-525.

Ervin, S. (1961). Changes with age in verbal determinants of word association. American Journal of Psychology, 74, 361-372.

Fabricius, W. V., \& Cavalier, L. (1989). The role of causal theories about memory in young children`s memory strategy choice. Child Development, 60, 298-308.

Fabricius, W. V., \& Hagen, J. W. (1984). The use of causal attributions about recall performance to assess metamemory and predict strategic memory behavior in young children. Developmental Psychology, 20, 975-987.

Fenson, L., Vella, D., \& Kennedy, M. (1989). Children's knowledge of thematic and taxonomic relations at two years of age. Child Development, 60, 911-919.

Fernandez-Duque, D., Baird, J. A., \& Posner, M. I. (2000). Executive attention and metacognitive regulation. Consciousness and Cognition, 9(2), 288-307.

Fischer, K. W. (1980). A theory of cognitive development: The control and construction of hierarchies of skills. Psychological Review, 87, 477-531.

Flavell, J. H. (1970). Developmental studies of mediated memory. In H. W. R. L. P. Lipsitt (Ed.), Advances in child development an behavior (Vol. 5, pp. S. 181-211). New York: Academic Press.

Flavell, J. H. (1971). What is memory development the development of? Human Development, 14, 272-278.

Flavell, J. H. (1978a). Comments. In R. S. Siegler (Ed.), Children's thinking: What develops? (pp. 97-105). 
Flavell, J. H. (1978b). Metacognitive development. In J. M. S. C. J. Brainerd (Ed.), Structural process theories of complex human behavior (pp. S. 213-245). Alphen a.d. Rijn: Sijthoff \& Noordhoff.

Flavell, J. H. (1981). Cognitive monitoring. In W. P. Dickson (Ed.), Children `s oral communication skills (pp. 35-60). New York: Academic Press.

Flavell, J. H., Beach, D. R., \& Chinsky, J. M. (1966). Spontaneous verbal rehearsal in a memory task as a function of age. Child Development, 37(2), 283-299.

Flavell, J. H., Friedrichs, A. G., \& Hoyt, J. D. (1970). Developmental changes in memorization processes. Cognitive Psychology, 1, 324-340.

Flavell, J. H., Miller, P. H., \& Miller, S. H. (1993). Cognitve development. Eaglewood Cliffs, NJ: Prentice Hall.

Flavell, J. H., \& Wellman, H. M. (1977). Metamemory. In R. V. K. J. W. Hagen (Ed.), Perspectives in the development of memory and cognition. (pp. 3-33). Hillsdale, NJ: Erlbaum.

Folds, T. H., Footo, M. M., Guttentag, R. E., \& Ornstein, P. A. (1990). When children mean to remember: Issues of context specifity, strategy effectiveness, and intentionality in the development of memory. In D. F. Bjorklund (Ed.), Children's strategies. Contemporary views of cognitive development. (pp. 67-91). Hillsdale, N.J.: Erlbaum.

Frankel, M. T., \& Rollins, H. A. (1985). Associative and categorical hypotheses of organization in the free recall of adults and children. Journal of Experimental Child Psychology, 40, 304-318.

Furth, H. G., \& Milgram, N. A. (1973). Labeling and grouping effects in the recall of pictures by children. Child Development, 44, 511-518.

Gathercole, S. E., Adams, A. M., \& Hitch, G. J. (1994). Do young children rehearse? An individual-differences analysis. Memory and Cognition, 22(2), 201-207.

Gaultney, J. F., Bjorklund, D. F., \& Goldstein, D. (1996). To be young, gifted, and strategic: Advantages for memory performance. Journal of Experimental Child Psychology, 61(1), 43-66.

Geary, D. C., Klosterman, I. H., \& Adrales, K. (1989). Metamemory and academic achievement: Testing the validity of a group-administered metamemory battery. The Journal of Genetic Psychology, 151(4), 439-450.

Gelabert, T., Torgesen, J. K., Dice, C., \& Murphy, H. (1980). The effects of situational variables on the use of rehearsal by first-grade children. Child Development, 51(3), 902-905. 
Gelman, R., \& Baillargeon, R. (1983). A review of some Piagetian concepts. In J. H. F. E. M. Markman (Ed.), Handbook of child psychology (Vol. 3, pp. S. 167-230). New York: Wiley.

Gerjuoy, I. R., \& Spitz, H. H. (1966). Associative clustering in free recall: Intellectual and developmental variables. American Journal of Experimental Child Psychology, 33, 504-513.

Ghatala, E. S. (1984). Developmental changes in incidental memory as a function of meaningfulness and encoding condition. Developmental Psychology, 20(2), 208-211.

Ghatala, E. S., \& Levin, J. R. (1981). Children's incidental memory for pictures: Item processing versus list organization. Journal of Experimental Child Psychology, 33, 504-513.

Ghatala, E. S., Levin, J. R., Pressley, M., \& Goodwin, D. (1986). A componential analysis of the effects of derived and supplied strategy-utility information on children's strategy selection. Journal of Experimental Child Psychology, 41, 76-92.

Glass, A. L., Holaoak, K. J., \& Kossan, N. E. (1977). Children's ability to detect semantic contradictions. Child development, 48, 279-283.

Goldman, A. E., \& Levine, M. A. (1963). A developmental study of object sorting. Child Development, 34, 649-666.

Greene, T. R. (1989). Children's understanding of class inclusion hierarchies: The relationship between external representation between task and performance. Journal of Child Psychology, 48(62-89).

Guttentag, R. E. (1984). The mental effort requirement of cumulative rehearsal: A developmental study. Journal of Experimental Child Psychology, 37, 92-106.

Guttentag, R. E., \& Lange, G. (1994). Motivational influences on strategic remembering. Learning \& Individual Differences, 6(3), 309-330.

Guttentag, R. E., \& Ornstein, P. A. (1990). Attentional capacity and children's memory strategy use. In J. T. Enns (Ed.), The development of attention: Research and theory (pp. 305-320). Amsterdam: Elsevier.

Guttentag, R. E., Ornstein, P.A. \& Siemens, L. (1987). Children's spontaneous rehearsal: Transitions in strategy acquisition. Cognitive Development, 2, 307-326.

Hacker, D. J. (1998). Definitions and Empirical Foundations. In D. J. Hacker, Dunsosky, J.\& Graesser, A.C: (Ed.), Metacognition in Educational Theory and Practice. MAhwah: Lawrence Erlbaum Ass. 
Hagen, J. W., \& Kingsley, P. R. (1968). Labeling effects in short-term memory. Child Development, 39(1), 113-121.

Hager, W. (1992a). Jenseits von Experiment und Quasi-Experiment. Zur Struktur psychologischer Versuche und zur Ableitung von Vorhersagen.Unpublished manuscript, Göttingen.

Hager, W. (1992b). Eine Strategie zur Entscheidung über psychologische Hypothesen. Psychologische Rundschau, 43(1), 18-29.

Hale, S. (1990). A global developmental trend in cognitive processing speed. Child development, 61, 653-663.

Hasher, L., \& Clifton, D. (1974). A developmental study of attribute encoding in free recall. Journal of Experimental Child Psychology, 17, 332-346.

Hasselhorn, M. (1986). Differentielle Bedingungsanalyse verbaler Gedächtnisleistungen bei Schulkindern. Frankfurt/M.: Lang.

Hasselhorn, M. (1990a). Kategoriales Organisieren als Gedächtnisstrategie: Allgemeine und differentielle Entwicklungsperspektiven im Grundschulalter. In M. Knopf \& W. Schneider (Eds.), Entwicklung. Allgemeine Verläufe - Individuelle Unterschiede Pädagogische Konsequenzen. (pp. 117-143). Göttingen: Hogrefe.

Hasselhorn, M. (1990b). The emergence of strategic knowledge activation in categorical clustering during retrieval. Journal of Experimental Child Psychology, 50, 59-80.

Hasselhorn, M. (1992). Metakognition und Lernen. In G. Nold (Ed.), Lernbedingungen und Lernstrategien: Welche Rolle spielen kognitive Verstehensstrukturen? (pp. 35-63). Tübingen: Gunter Narr.

Hasselhorn, M. (1994). Zur Erfassung von Metagedächtnisaspekten bei Grundschulkindern. Zeitschrift für Entwicklungspsychologie und Pädagogische Psychologie, 26(1), 71-78.

Hasselhorn, M. (1995). Beyond production deficiency and utilization inefficiency: Mechanisms of the emergence of strategic categorization in episodic memory tasks. In F. E. Weinert \& W. Schneider (Eds.), Memory Development and Competencies: Issues in Growth and Development (pp. 141-159). Mahwah, N. J.: Lawrence Erlbaum.

Hasselhorn, M. (1996). Kategoriales Organisieren bei Kindern. Zur Entwicklung einer Gedächtnisstrategie. Göttingen: Hogrefe.

Hasselhorn, M. (2001). Metakognition. In D. H. Rost (Ed.), Handwörterbuch Pädagogische Psychologie (Vol. 2, pp. 466-471). Weinheim: Beltz, Psychologie Verlags Union. 
Hasselhorn, M., Hager, W., \& Baving, L. (1989). Zur Konfundierung metakognitiver und motivationaler Aspekte im Prädiktionsverfahren. Zeitschrift für experimentelle und angewandte Psychologie, 36(1), 31-41.

Hasselhorn, M., Jaspers, A., \& Hernando, M.-D. (1994). Typizitätsnormen zu zehn Kategorien für Kinder von der Vorschule bis zur vierten Grundschulklasse. In W. Hager \& M. Hasselhorn (Eds.), Handbuch deutschsprachiger Wortnormen (pp. 102114). Göttingen: Hogrefe.

Hasselhorn, M., \& Lindner-Müller, C. (1995). Kategoriales Organisieren und kumulatives Rehearsal: Zur Entwicklung der kombinierten Nutzung zweier Gedächtnisstrategien. Zeitschrift für Entwicklungspsychologie und Pädagogische Psychologie, 27(2), 139156.

Hasselhorn, M., Mähler, C., \& Grube, D. (1995). Entwicklungsveränderungen und stabilitäten im Metagedächtnis während der Grundschuljahre. Empirische Pädagogik, 9, 33-53.

Hau, K.-T., Kong, C.-K., \& Marsh, H. W. (2000). Extension of the Internal/external Frame of Reference Model of Self-Concept Formation: Importance of Native and Konnotive Language For Chinese Students.Unpublished manuscript.

Haynes, C. R., \& Kulhavy, R. W. (1976). Conservation level and category clustering. Developmental Psychology, 12, 179-184.

Helmke, A. (1991). Entwicklung des Fähigkeitsbildes vom Kindergarten bis zur dritten Klasse. In R. Pekrun \& H. Fend (Eds.), Schule und Persönlichkeitsentwicklung. Ein Resumee der Längsschnittforschung (pp. 33-56). Stuttgart: Enke.

Helmke, A. (1998). Vom Optimisten zum Realisten? Zur Entwicklung des Fähigkeitsselbstkonzeptes vom Kindergarten bis zur 6. Klassenstufe. In F. E. Weinert (Ed.), Entwicklung im Kindesalter (pp. 115-132). Weinheim: PVU.

Helmke, A. (1999). From optimism to realism? Development of children's academic selfconcept from kindergarten to grade 6. In F. E. S. Weinert, W. (Ed.), Individual Development from 3 to 12 (Vol. 198-221). Cambridge: Cambridge University Press.

Helmke, A., \& Rheinberg, F. (1996). Anstrengungsvermeidung - Morphologie eines Konstrunktes. In U. K.-K. P. D. C. Spiel (Ed.), Motivation und Lernen aus der Perspektive lebenslanger Entwicklung. (pp. 207-244). Münster: Waxmann.

Helmke, A., \& van Aken, M. A. G. (1995). The causal ordering of academic achievement and self-concept of ability during elementary school: A longitudinal study. Journal of Educational Psychology, 87, 624-637. 
Horowitz, A. B. (1969). Effects of stimulus presentation modes on children's recall and clustering. Psychonomic Science, 14, 297-298.

Howard, M. W., \& Kahana, M. J. (2001). A distributed representation of temporal context. Journal of Mathematical Psychology., 46, 269-299.

Howe, M. L., Brainerd, C. J., \& Kingma, J. (1985). Development of organization in recall: A stages-of-learning analysis. Journal of Experimental Child Psychology, 39, 230-251.

Howe, M. L., \& O'Sullivan, J. T. (1990). The development of strategic memory: Coordinating knowledge, Metamemory, and ressources. In D. F. Bjorklund (Ed.), Children's strategies: Contemporary views of cognitive development (pp. S. 129-155). Hillsdale, NJ: Erlbaum.

Hulme, C., Thomson, N., Muir, C., \& Lawrence, A. (1994). Speech rate and the development of short-term memory span. Journal of Experimental Child Psychology, 38, 241-253.

Jacobs, J. E., Lanza, S., Osgood, D. W., Eccles, J. S., \& Wigfield, A. (2002). Changes in children's self-competence and values: Gender and domain differences across grades one through twelve. Child Development, 73(2), 509-527.

Jenkins, J. J., Mink, W. D., \& Russell, W. A. (1958). Associative clustering as a function of verbal association strength. Psychological Reports, 4, 127-136.

Joyner, M. H., \& Kurtz-Costes, B. E. (1997). Metamemory development. In N. Cowan (Ed.), The development of memory. (pp. 275-300). London: UCL Press.

Justice, E. M. (1985). Categorization as a preferred memory strategy: Developmental changes during elementary school. Developmental Psychology, 21(6), 1105-1110.

Justice, E. M. (1986). Developmental changes in judgements of relative strategy effectiveness. British Journal of Developmental Psychology, 4, 75-81.

Kail, R. (1979). Die Entwicklung des Gedächtnisses. In L. Montada (Ed.), Brennpunkte der Entwicklungspsychologie (pp. 77-89). Stuttgart: Kohlhammer.

Kail, R., \& Salthouse, T. A. (1994). Processing speed as a mental capacity. Acta Psychologica, 86, 199-225.

Kalish, C. W. (2002). Essentialist to some degree: Beliefs about the structure of natural kind categories. Memory \& Cognition, 30(3), 340-352.

Kee, D. W. (1994). Developmental differences in associative memory: Strategy use, mental effort, and knowledge access interactions. In H. W. Reese (Ed.), Advances in child development and behavior. (Vol. 25, pp. 7-32). California State: Fullerton. 
Kee, D. W., \& Bell, T. S. (1981). The development of organizational strategies in the storage and retrieval of categorical items in free-recall learning. Child Development, 52, 1163 1171.

Kee, D. W., \& Davis, L. (1988). Mental effort and elaboration. A developmental analysis. Contemporary Educational Psychology, 13, 221-228.

Kee, D. W., \& Davis, L. (1990). Mental effort and elaboration: Effects of accessibility and instruction. Journal of Experimental Child Psychology, 49, 264-274.

Kendler, H. H., \& Kendler, T. S. (1962). Vertical and horizontal processes in problem solving. Psychological Review, 69, 1-16.

Kendler, T. S. (1972). An ontogeny of mediational deficiency. Child Development, 43, 1-17.

Kluwe, R. H., Wolke, D., \& Bunge, B. (1982). Zur kategorialen Organisation semantischer Informationen bei 10jährigen Kindern und bei Erwachsenen. Sprache \& Kognition, 1, $15-26$.

Knopf, M., Körkel, J., Schneider, W., \& Weinert, F. E. (1988). Human memory as a faculty versus human memory as a set of specific abilities: Evidence from a life-span approach. In F. E. W. M. Perlmutter (Ed.), Memory development: Universal cahnges and individual differences. (pp. 331-352). Hillsdale, NJ: Lawrence Erlbaum Associates.

Knopf, M., \& Schneider, W. (1998). Die Entwicklung des kindlichen Denkens und die Verbesserung der Lern- und Gedächtniskompetenzen. In F. E. Weinert (Ed.), Entwicklung im Kindesalter. Weinheim: Beltz, Psycholgie Verlags Union.

Kobasigawa, A. (1974). Utilization of retrieval cues by children in recall. Child Development, 45(1), 127-134.

Kobasigawa, A. (1977). Retrieval strategies in the development of memory. In R. V. K. J. W. Hagen (Ed.), Perspective on the development of memory and cognition (pp. 177-201). Hillsdale, NJ: Lawrence Erlbaum Associates.

Kobasigawa, A., \& Middleton, D. B. (1972). Free recall of categorized items by children at three grade levels. Child Development, 43, 1067-1072.

Kobasigawa, A., \& Orr, R. R. (1973). Free recall and retrieval speed of categorized items by Kindergarten children. Journal of Experimental Child Psychology, 15, 187-192.

Koriat, A. (2000). The feeling of knowing: Some metatheoretical implications for consciousness and control. Consciousness and Cognition, 9, 149-171. 
Koriat, A., \& Goldsmith, M. (1998). The role of metacognitive processes in the regulation of memory performance. In G. N. Mazzoni \& T. O. Nelson (Eds.), Metacognition and cognitive Neuropsychology: Monitoring and control Processes (pp. 97-118). Mahwah, N. J.: Lawrence Erlbaum.

Körkel, J. (1987). Die Entwicklung von Gedächtnis- und Metagedächtnisleistungen in Abhängigkeit von bereichsspezifischen Vorkenntnissen. Frankfurt a.M.: Lang.

Krapp, A. (1997a). Selbstkonzept und Leistung - Dynamik ihres Zusammenspiels: Literaturüberblick. In F. E. Weinert \& A. Helmke (Eds.), Entwicklung im Grundschulalter. Weinheim: Beltz, Psychologie Verlags Union.

Krapp, A. (1997b). Selbstkonzept und Leistung - Dynamik ihres Zusammenspiels: Literaturüberblick. In F. E. W. A. Helmke (Ed.), Entwicklung im Grundschulalter (pp. 325-339). Weinheim: Beltz, PVU.

Kreutzner, M. A., Leonard, L. A., \& Flavell, J. H. (1975). An interview study of children's knowledge about memory. Monographs of the sociaty for Research in child development, 40(1, No 159).

Krist, H., \& Wilkening, F. (1991). Repräsentationale Entwicklung. Sprache \& Kognition, 10, 181-195.

Kuhn, D. (2000a). Metacognitive development. Current Directions in Psychological Science, 9(5), 178-181.

Kuhn, D. (2000b). Does memory development belong on an endangered topic list? Child Development, 71(1), 21-25.

Kunzinger, E. L., \& Witryol, S. L. (1984). The effects of differential incentives on secondgrade rehearsal and free recall. The Journal of Genetic Psychology, 144, 19-30.

Kurtz, B. E., \& Borkowski, J. C. (1987). Development of strategic skills in impulsive and reflective children: A developmental study of metacognition. Journal of Experimental Child Psychology, 43, 129-148.

Kurtz, B. E., Borkowski, J. C., \& Deshmukh, K. (1988). Metamemory development in Maharashtran children: Influences from home and school. Journal of Genetic Psychology, 149, 363-376.

Kurtz, B. E., \& Borkowski, J. G. (1984). Children's metacognition: Exploring relations among knowledge, process, and motivational variables. Journal of Experimental Child Psychology., 37, 335-354. 
Kurtz, B. E., Schneider, W., Carr, M., Borkowski, J. G., \& Turner, L. A. (1988). Sources of memory and metamemory development: Societal, parental, and educational influences. In P. M. S. S. M. Gruneberg (Ed.), Practical aspects of memory (pp. 537 542). New York: Wiley.

Kurtz, B. E., \& Weinert, F. E. (1989). Metamemory, memory performance, and causal attributions in gifted and average children. Journal of Experimental Child Psychology, 48(1), 45-61.

Lange, G. (1973). The development of conceptual and rote recall skills among school age children. Journal of Experimental Child Psychology, 15, 394-406.

Lange, G. (1978). Organization-Related Processes in Children's Recall. In P. A. Ornstein (Ed.), Memory development in children. Hillsdale, N. J.: Lawrence Erlbaum.

Lange, G., \& Griffith, S. (1990). Form characteristics of category-retrieval relationships in children and adults. The Journal of General Psychology, 117(1), 5-14.

Lange, G., \& Griffith, S. B. (1977). The locus of organization failures in children's recall. Child Development, 48, 1498-1502.

Lange, G., Guttentag, R. E., \& Nida, R. E. (1990). Relationships between study organization, retrieval organization, and general and strategy-specific memory knowledge in young children. Journal of Experimental Child Psychology, 49, 126-146.

Lange, G., \& Hultsch, D. F. (1970). The development of free classification and free recall in children. Developmental Psychology, 3, 408.

Lange, G., \& Jackson, P. (1974). Personal organization in children's free recall. Child Development, 45, 1060-1067.

Lange, G., \& Pierce, S. H. (1992). Memory-strategy learning and maintenance in preschool children. Developmental Psychology, 28, 453-462.

Laurence, M. W. (1966). Age differences in organization and recall: The effects of training in categorization. Canadian Journal of Psychology, 20, 388-399.

Leal, L., Crays, N., \& Moely, B. E. (1985). Training children to use a self-monitoring study strategy in preparation for recall: Mainetance and generalization effects. Child Development, 56, 643-653.

LeBlanc, R. S., Muise, J. G., \& Blanchard, L. (1992). Backward masking in children and adolescence: Sensory transmission, accrual rate and asymptotiv performance. Journal of Experimental Child Psychology, 53, 105-114. 
Lepola, J., \& Rauhanummi, T. (2000). Developmental profiles of motivation in elementary school years and their cognitive consequences. In J. Lepola (Ed.), Motivation inearly school years: Developmental Patterns and Cognitive Consequences (pp. 1-23). Turku: Turun Yliopiston.

Liberty, C., \& Ornstein, P. A. (1973). Age differences in organization and recall: The effects of training in categorization. Journal of Experimental Child Psychology, 15, 169-186.

Lindberg, M. A. (1980). Is knowledge base development a necessary and sufficient condition for memory development? Journal of Experimental Child Psychology, 30, 401-410.

Lindberg, M. A. (1991). A taxonomy of suggestibility and eyewitness memory: Age, memory process, and focus of analysis. In J. L. Doris (Ed.), The suggestibility of children `s recollections.

Lockhart, R. S. (2000). Methods of memory research. In E. Tulving \& F. J. M. Craik (Eds.), The Oxford Handbook of Memory (pp. 45-57). Oxford: University Press.

Loughlin, K. A., \& Daehler, M. W. (1973). The effects of distraction and added perceptual cues on the delayed reaction of very young children. Child Development, 44(2), 384388.

Mandler, G. (1967). Organization and memory. In K. W. S. a. J. T. Spence (Ed.), The psychology of learning and motivation (Vol. 1, pp. 327-372). New York: Academic Press.

Mandler, G., \& Stephens, D. (1967). The development of free and constrained conceptualization and subsequent verbal memory. Journal of Experimental Child Psychology, 5, 86-93.

Mandler, J. M., \& Bauer, P. J. (1988). The cradle of categoration: Is basic level basic? Cognitive Development, 3, 247-264.

Mandler, J. M., Fivush, R., \& Reznick, J. S. (1987). The development of contextual categories. Cognitive Development, 2, 339-354.

Mansfield, A. F. (1977). Semantic organization in the young child: Evidence for the development of semantic feature systems. Journal of Experimental Child Psychology, 23, 57-77.

Markman, E. M. (1989). Categorisation and naming in children. Cambridge, MA: Cambridge University.

Markman, E. M., \& Callanan, M. A. (1984). An analysis of hierarchical classification. In R. J. Sternberg (Ed.), Advances in the psychology of human intelligence. (Vol. 2, pp. 325365). Hillsdale, NJ: Erlbaum. 
Markman, E. M., Cox, B., \& Machida, S. (1981). The standard object sorting task as a measureof conceptual organization. Developmental Psychology, 17, 115-117.

Marsh, H. W. (1986). Verbal and math self-concepts: An internal/external frame of reference model. American Educational Research Journal, 23(1), 129-149.

Marsh, H. W. (1988). Causal effects of academic self-concept on academic achievement: A reanalysis of Newman (1984). Journal of Experimental Education, 52(2), 100-103.

Marsh, H. W. (1990a). Causal ordering of academic self-concept and academic achievement: A multiwave, longitudinal panel analysis. Journal of Educational Psychology, 82(4), 646-656.

Marsh, H. W. (1990b). Influences of internal and external frames of reference on the formation of math and English self-concepts. Journal of Educational Psychology, 82(1), 107-116.

Marsh, H. W. (1990c). The structure of academic self-concept: The Marsh/Shavelson model. Journal of Educational Psychology, 82(4), 623-636.

Marsh, H. W., Byrne, B. M., \& Shavelson, R. J. (1988). A multifaceted academic selfconcept: Its hierarchical structure and its relation to academic achievement. Journal of Educational Psychology, 80(3), 366-380.

Marsh, H. W., Craven, R., \& Debus, R. (1998). Structure, stability, and development of young children's self-concepts: A multicohort-multioccasion study. Child Development, 69(4), 1030-1053.

Marsh, H. W., Craven, R. G., \& Debus, R. (1991). Self-concepts of young children 5 to 8 years of age: Measurement and multidimensional structure. Journal of Educational Psychology, 83(3), 377-392.

Marsh, H. W., Ellis, L. A., \& Craven, R. G. (2002). How do preschool children feel about themselves? Unraveling measurement and multidimensional self-concept structure. Developmental Psychology, 38(3), 376-393.

Marsh, H. W., Kong, C.-K., \& Hau, K.-T. (2001). Extension of the internal/external frame of reference model of self-concept formation: Importance of native and nonnative languages for Chinese students. Journal of Educational Psychology, 93(3), 543-553.

Marsh, H. W., \& Yeung, A. S. (1998). Top-down, bottom-up, and horizontal models: The direction of causality in multidimensional, hierarchical self-concept models. Journal of Personality and Social Psychology, 75(2), 509-527. 
Masur, E. F., McIntyre, C. W., \& Flavell, J. H. (1973). Developmental changes in apportionmentof study time among items in a multitrial free recall task. Journal of Experimental Child Psychology, 15, 237-246.

McFarland, C. E., \& Kellas, G. (1975). Category similarity effects in children's semantic memory retrieval. Journal of Experimental Child Psychology, 20, 369-376.

McGilly, K., \& Siegler, R. S. (1989). How children choose among serial recall strategies. Child Development, 60(1), 172-182.

McGilly, K., \& Siegler, R. S. (1990). The influence of encoding and strategic knowledge on children's choices among serial recall strategies. Developmental Psychology, 26(6), 931-941.

Melkman, R., \& Deutsch, C. (1977). Memory functioning as related to developmental changed in bases of organization. Journal of Experimental Child Psychology, 23, 8497.

Melkman, R., Tversky, B., \& Baratz, D. (1981). Developmental trends in the use of perceptual and conceptual attributes in grouping, clustering, and retrieval. Journal of Experimental Child Psychology, 31, 470-486.

Metcalfe, J. (2000). Metamemory: Theory and data. In E. Tulving \& F. J. M. Craik (Eds.), The Oxford Handbook of Memory (pp. 197-211). Oxford: University Press.

Miller, G. A. (1956). The magical number seven, plus or minus two: Some limits on our capacity for processing information. Psychological Review, 63, 81-97.

Miller, G. A., Galanter, E., \& Pribram, K. H. (1960). Plans and the structure of behavior. New York: Holt.

Miller, P. H. (1990). The development of strategies of selective attention. In D. F. Bjorklund (Ed.), Children's Strategies: Contemporary Views of cognitive Development (pp. 157184). Hillsdale, N. J.: Lawrence Erlbaum.

Miller, P. H. (1994). Individual differences in children's strategic behavior: Utilization deficiencies. Learning and Individual Differences, 6, 285-307.

Miller, P. H. (2000). How best to utilize a deficiency. Child Development, 71(4), 1013-1017.

Miller, P. H., \& Aloise-Young, P. A. (1995). Preschoolers' strategic behavior and performance on a same-different task. Journal of Experimental Child Psychology, 60, 284-303.

Miller, P. H., \& Coyle, T. R. (1999). Developmental change: Lessons from microgenesis. In E. K. Scholnick, K. Nelson, S. Gelman \& P. Miller (Eds.), Conceptual Development: Piaget's Legacy. Mahwah, N. J.: Lawrence Erlbaum. 
Miller, P. H., DeMarie-Dreblow, D., \& Woody-Ramsey, J. (1986). Children's strategies for gathering information in three tasks. Child Development, 57, 1429-1439.

Miller, P. H., \& Harris, Y. R. (1988). Preschoolers' strategies of attention on a same-different task. Developmental Psychology, 24, 628-633.

Miller, P. H., \& Seier, W. L. (1994). Strategy utilization deficiencies in children: When, where and why. In H. W. Reese (Ed.), Advances in Child Development and Behavior (Vol. 25, pp. 107-156). New York: Academic Press.

Miller, P. H., Seier, W. L., Barron, K. L., \& Probert, J. S. (1994). What causes a memory strategy deficiency? Cognitive Development, 9, 77-101.

Miller, P. H., Seier, W. L., Probert, J. S., \& Aloise, P. A. (1991). Age differences in the capacity demands of a strategy among spontaneously strategic children. Journal of Experimental Child Psychology, 52, 149-165.

Miller, P. H., Woody-Ramsey, J., \& Aloise, P. A. (1991). The role of strategy effortfulness in strategy effectiveness. Developmental Psychology, 27, 738-745.

Mitchell, D. B., \& Hunt, R. R. (1989). How much "effort" should be devoted to memory? Memory \& Cognition, 17, 337-348.

Moely, B. E., \& Jeffrey, W. E. (1974). The effect of organization training on children's free recall of category items. Child Development, 45, 135-143.

Moely, B. E., Olson, F. A., Halwes, T. G., \& Flavell, J. H. (1969). Production deficiency in young children's clustered recall. Developmental Psychology, 1, 26-34.

Moely, B. E., \& Shapiro, S. I. (1971). Free recall and clustering at four age levels: Effects of learning to learn and presentation method. Developmental Psychology, 4, 490.

Möller, J., \& Köller, O. (2001). Dimensional comparisons: An experimental approach to the internal/external frame of reference model. Journal of Educational Psychology, 93(4), 826-835.

Moschner, B. (2001). Selbstkonzept. In D. H. Rost (Ed.), Handbuch Pädagogische Psychologie. Weinheim: Beltz, Psychologie Verlags Union.

Moses, L. J., \& Baird, J. A. (1999). Metacognition. In R. A. W. F. C. Keil (Ed.), The MIT encyclopedia of cognitive science. Cambridge, M.A.: MIT Press.

Murphy, M. D. (1979). Measurement of category clustering in free recall. In C. Puff (Ed.), Memory organization and structure. (pp. 51-83). New York: Academic Press.

Murphy, M. D., \& Puff, C. R. (1982). Free Recall: Basic Methodology and Analyses. In C. R. Puff (Ed.), Handbook of Research Methods in Human Memory and Cognition. New York: Academic Press. 
Naus, M. J., Ornstein, P. A., \& Aivano, S. (1977). Developmental changes in memory: The effects of processing time and rehearsal instructions. Journal of Experimental Child Psychology, 23, 237-251.

Neimark, E., Slotnick, N. S., \& Ulrich, T. (1971). Development of memorization strategies. Developmental Psychology, 5, 427-432.

Nelson, K. (1969). The organization of free recall by young children. Journal of Experimental Child Psychology, 8, 284-295.

Nelson, K., \& Narens, L. (1990). Metamemory: A theoretical framework and new findings. In G. Bower (Ed.), The psychology of learning and motivation (pp. 125-173). New York: Academic Press.

Nelson, T., \& Narens, L. (1994). Why Investigate Metacognition? In J. M. A. P. Shiamura (Ed.), Metacognition: Knowing about knowing. Cambridge, Massachusetts: MIT Press.

Newman, L. S. (1990). Intentional and unintentional memory in young children: Remembering vs. playing. Journal of Experimental Child Psychology, 50(2), 243-258.

Nichols, J. (1979). Development of perception of attainment and causal attributions for sucess and failure in reading. Journal of Educational Psychology(71), 94-99.

Norman, D. A., \& Bobrow, D. G. (1979). Descriptions: An intermediate stage in memory retrieval. Cognitive Psychology, 11, 107-123.

Ornstein, P. A. (1999). Comments: Toward an understanding of the development of memory. In F. E. S. Weinert, W. (Ed.), Individual development from 3 to 12: Findings from the Munich Longitudinal Study (pp. 94-105). Cambridge: Cambridge University Press.

Ornstein, P. A., Baker-Ward, L., \& Naus, M. J. (1988). The development of mnemonic skill. In F. E. Weinert \& M. Perlmutter (Eds.), Memory Development: Universal Changes and Individual Differences (pp. 31-51). Hillsdale, N. J.: Lawrence Erlbaum.

Ornstein, P. A., \& Haden, C. A. (2001). Memory development or the development of memory. Current Directions in Psychological Science, 10(6), 202-205.

Ornstein, P. A., Hale, G. A., \& Morgan, J. S. (1977). Developmental differences in recall and output organization. Bulletin of the Psychonomic Society, 9, 29-32.

Ornstein, P. A., \& Naus, M. J. (1978). Rehearsal processes in children's memory. In P. A. Ornstein (Ed.), Memory Development in Children (pp. 69-99). Hillsdale, N. J.: Lawrence Erlbaum. 
Ornstein, P. A., \& Naus, M. J. (1983). "Rehearsing" according to artificially generated rehearsal patterns: An analysis of active rehearsal. Bulletin of the Psychonomic Society, 21(6), 419-422.

Ornstein, P. A., \& Naus, M. J. (1985). Effects of the knowledge base on children's memory strategies. Advances in Child Development and Behavior, 19, 113-148.

Ornstein, P. A., Naus, M. J. \& Liberty, C. (1975). Rehearsal and organizational processes in children's memory. Child Development, 46, 818-830.

Paris, S. G. (1988). Motivated remembering. In F. E. W. M. Perlmutter (Ed.), Memory development: Universal changes and individual differences. (pp. 221-242). Hillsdale, NJ: Erlbaum.

Paris, S. G., \& Lindauer, B. K. (1982). The development of cognitive skills during childhood.

In B. Woolman (Ed.), Handbook of developmental psychology (pp. 333-349). Englewood Cliffs, NJ: Prentice-Hall.

Paris, S. G., Lipson, M. Y., \& Wixson, K. K. (1983). Becoming a strategic reader. Contemporary Educational Psychology, 34, 293-316.

Paris, S. G., Newman, L. S., \& McVey, K. A. (1982). Learning in the functional significance of mnemonic actions: A microgenitic study of strategy acquisition. Journal of Experimental Child Psychology, 34, 490-509.

Paris, S. G., \& Oka, E. R. (1986). Children's reading strategies, metacognition, and motivation. Developmental Review, 6(1), 25-56.

Patterson, K. E., Meltzer, R. H., \& Mandler, G. (1971). Inter-response times in categorized free recall. Journal of Verbal learning \& Verbal Behavior, 10, 417-426.

Pekrun, R. (1997). Selbstkonzept und Leistung - Dynamik ihres Zusammenspiels: Kommentar. In F. E. Weinert \& A. Helmke (Eds.), Entwicklung im Grundschulalter (pp. 351-358). Weinheim: Beltz, Psychologie Verlags Union.

Pellegrino, J. W. (1975). A reply to Frender and Doubilet on the measurement of clustering. Psychological Bulletin, 82, 66-67.

Piaget, J. (1971). Biology and knowledge. Chicago: University of Chicago Press.

Pollio, H. R., Richards, S., \& Lucas, R. (1969). Temporal properties of category recall. Journal of Verbal learning \& Verbal Behavior, 8, 529-536.

Posnansky, C. J. (1978a). Age- and task-related differences in the use of category-size information for the retrieval of categorized items. Journal of Experimental Child Psychology, 26, 373-382. 
Posnansky, C. J. (1978b). Category norms for verbal items in25 categories for children in grades 2-6. Behavior Research Methods and Instruments, 10, 819-832.

Pressley, M. (1982). Elaboration and memory development. Child Development, 53(2), 296309.

Pressley, M., Borkowski, J. C., \& Schneider, W. (1989). Good information processing: What it is and how education can promote it. International Journal of Educational Research., 14, 857-867.

Pressley, M., Borkowski, J. G., \& O`Sullivan, J. T. (1985). Children`s metamemory and the teaching of memory strategies. In G. E. M. T. G. W. D.L. Forrest-Pressley (Ed.), Metacognition, cognition, and human performance (Vol. 1, pp. 111-153). Orlande, FL: Academic Press.

Pressley, M., Borkowski, J. G., \& Schneider, W. (1987). Cognitive strategies: Good strategy users coordinate metacognition and knowledge. In R. V. G. Whitehurst (Ed.), Annals of Child Development. (Vol. 5, pp. 89-129). New York, NY: JAI Press.

Pressley, M., Forrest-Pressley, D. L., Elliott-Faust, D., \& Miller, G. (1985). Children's use of cognitive strategies, how to teach strategies, and what to do if they can't be taught. In M. Pressley \& C. J. Brainerd (Eds.), Cognitive learning and memory in children. (pp. 1-47). New York: Springer.

Pressley, M., Levin, J. R., Ghatala, E. S., \& Ahmad, M. (1987). Test monitoring in young grade school children. Journal of Experimental Child Psychology, 43, 96-111.

Pressley, M., Ross, K. A., Levin, J. R., \& Ghatala, E. S. (1984). The role of strategy utility knowledge in children's strategy decision making. Journal of Experimental Child Psychology, 38, 491-504.

Pressley, M., Ross, K. A., Levin, J. R., \& Ghatala, E. S. (1985). The role of strategy utility knowledge in children's strategy decision making. Journal of Experimental Child Psychology, 38, 275-288.

Pressley, M., \& Schneider, W. (1997). Introduction to memory: Development during childhood and adolescence. Mahwah, N.J.: Erlbaum.

Quillian, M. R. (1968). Semantic memory. In M. Minsky (Ed.), Semantic information processing. (pp. 227-270). Cambridge, MA: MIT Press.

Quinn, P. C., \& Eimas, P. D. (2000). The emergence of category representations during infancy: Are separate perceptual and conceptual processes required? Journal of Cognition and Development, 1(1), 55-61. 
Rabinowitz, M. (1984). The use of categorical organisation: Not an all-or-none situation. Journal of Experimental Child Psychology, 38, 338-351.

Rabinowitz, M. (1991). Semantic and strategic processing: Independend roles in determining memory performance. Americal Journal of Psychology, 104, 427-437.

Rabinowitz, M., \& Chi, M. T. H. (1987). An interactive model of strategic processing. In S. J. Ceci (Ed.), Handbook of cognitive, social, and neuropsychological aspects of learning disabilities. (Vol. 2, pp. S. 83-102). Hillsdale, NJ: Erlbaum.

Rabinowitz, M., Freeman, K., \& Cohen, S. (1992). Use and maintenance of strategies: The influence of accessibility to knowledge. Journal of Educational Psychology, 84, 211218.

Rabinowitz, M., \& Kee, D. W. (1994). A framework for understanding individual differences in memory. In P. A. Vernon (Ed.), The neuropsychology of individual differences. (pp. 135-148).

Ratner, H. H., \& Myers, N. A. (1980). Related picture cues and memory for hidden-object locations at age two. Child development, 51(2), 561-564.

Reese, H. W. (1962). Verbal mediators as a function of age level. Psychological Bulletin, 59, 502-509.

Reese, H. W. (1976a). Models of memory development. Human Development, 19, 291-303.

Reese, H. W. (1976b). The development of memory: Life-span perspectives. In H. W. Reese (Ed.), Advances in child development and behavior (Vol. 11, pp. 1990-1212). New York: Academic Press.

Renkl, A., Helmke, A., \& Schrader, F.-W. (1997). Schulleistung und Fähigkeitsbild Universelle Beziehungen oder kontextspezifische Zusammenhänge? Ergebnisse aus dem SCHOLASTIK-Projekt. In F. E. Weinert \& A. Helmke (Eds.), Entwicklung im Grundschulalter (pp. 373-383). Weinheim: Beltz, Psychologie Verlags Union.

Renner, G., \& Fricke, T. (2001). Der Hamburg-Wechsler-Intelligenztest für Kinder. Report Psychologie, 26(8), 460-481.

Reyna, V. F. (1996). Conceptions of Memory Development with Implications for Reasoning and Decision Making. Annals of Child Development, 12, 87-118.

Reyna, V. F., \& Brainerd, C. J. (1989). Output interference, generic resources, and cognitive development. Journal of Experimental Child Psychology, 47, 27-46.

Roenker, D. L., Thompson, C. P., \& Brown, S. C. (1971). Comparison of measures for the estimation of clustering in free recall. Psychological Bulletin, 76, 45-48. 
Rosner, S. R., \& Hayes, D. S. (1977). A developmental study of category item production. Child Development, 48, 1062-1065.

Rossi, E. L., \& Rossi, S. I. (1965). Concept utilization, serial order and recall in nursery school children. Child Development, 36, 771-778.

Rundus, D. (1971). Analysis of rehearsal processes in free recall. Experimental Psychology, 89(1), 63-77.

Salatas, H., \& Flavell, J. H. (1976a). Behavioral and metamnemonic indicators of strategic behaviors under remember instruction in first grade. Child Development, 47, 81-99.

Salatas, H., \& Flavell, J. H. (1976b). Retrieval of recently learned information: Development of strategies and control skills. Child Development, 47, 941-948.

Schacter, D. L., Wagner, A. D., \& Buckner, R. L. (2000). Memory systems of 1999. In E. Tulving \& F. J. M. Craik (Eds.), The Oxford Handbook of Memory (pp. 627-643). Oxford: University Press.

Schlagmüller, M., \& Schneider, W. (2002). The development of organizational strategies in children: Evidence from a microgenetic longitudinal study. Journal of Experimental Child Psychology, 81(3), 298-319.

Schlagmüller, M., Visé, M., \& Schneider, W. (2001). Zur Erfassung des Gedächtniswissens bei Grundschulkindern: Konstruktionsprinzipien und empirische Bewährung der Würzburger Testbatterie zum deklarativen Metagedächtnis. Zeitschrift für Entwicklungspsychologie und Pädagogische Psychologie, 33(2), 91-102.

Schnabel, K. U. (1997). Schulleistung und Fähigkeitsbild - Universelle Beziehungen oder kontextspezifische Zusammenhänge? Kommentar. In F. E. Weinert \& A. Helmke (Eds.), Entwicklung im Grundschulalter (pp. 385-388). Weinheim: Beltz, Psychologie Verlags Union.

Schneider, W. (1985b). Developmental trends in the metamemory-memory behavior relationship: An integrative review. In G. E. M. T. G. W. D. L. Forrest-Pressley (Ed.), Metacognition, cognition, and human performance. Orlando, Florida: Academic Press.

Schneider, W. (1986). The role of conceptual knowledge and metamemory in the development of organizational processes in memory. Journal of Experimental Child Psychology, 42, 218-236.

Schneider, W. (1989). Zur Entwicklung des Metagedächtnisses bei Kindern. Bern: Huber. 
Schneider, W. (1999). The development of metamemory knowledge in children. In D. G. A. Koriat (Ed.), Attention and performance XVIII: Cognitive regulation of performance interaction of theory and application (Vol. 487-514). Cambridge, MA: MIT Press.

Schneider, W. (2000). Research on memory development: Historical trends and current themes. International Journal of Behavioral Development, 24(4), 407-420.

Schneider, W. (2001). Gedächtnisentwicklung. In Handbuch Pädagogische Psychologie (pp. 194-200). Weinheim: Beltz Psychologie Verlags Union.

Schneider, W., \& Bjorklund, D. F. (1992). Expertise, aptitude, and strategic remembering. Child Development, 63, 461-473.

Schneider, W., \& Bjorklund, D. F. (1998). Memory. In W. Damon, D. Kuhn \& R. Siegler (Eds.), Handbook of Child Psychology (5th ed., Vol. 2, pp. 467-521). New York: Wiley.

Schneider, W., Bjorklund, D. F., \& Maier-Brückner, W. (1996). The effects of expertise and IQ on children's memory: When knowledge is, and when it is not enough. International Journal of Behavioral Development, 19(4), 773-796.

Schneider, W., Borkowski, J. G., Kurtz, B. E., \& Kerwin, K. (1986). Metamemory and motivation: A comparison of strategy use and performance in German and American children. Journal of Cross-Cultural Psychology, 17, 315-336.

Schneider, W., Bullock, M., \& Sodian, B. (1998). Die Entwicklung des Denkens und der Intelligenzunterschiede zwischen Kindern. In F. E. Weinert (Ed.), Entwicklung im Kindesalter. Weinheim: Beltz, Psychologie Verlags Union.

Schneider, W., Gruber, H., Gold, A., \& Opwis, K. (1993). Chess expertise and memory for chess positions in children and adults. Journal of Experimetnal Child Psychology, 56, 328-349.

Schneider, W., Körkel, J., \& Vogel, K. (1987). Zusammenhänge zwischen Metagedächtnis, strategischem Verhalten und Gedächtnisleistungen im Grundschulalter: Eine entwicklungspsychologische Studie. Zeitschrift für Entwicklungspsychologie und Pädagogische Psychologie, 19(2), 99-115.

Schneider, W., Körkel, J., \& Weinert, F. E. (1987). The effects of intelligence, self-concept, and attributional style on metamemory and memory behaviour. Internationsal Journal of Behavioral Development, 10, 281-299.

Schneider, W., Körkel, J., \& Weinert, F. E. (1989). Domain-specific knowledge and memory performace: A comparison of high- and low-aptitude children. Journal of Educational Psychology, 81, 306-312. 
Schneider, W., Kron, V., Hünnerkopf, M., \& Krajewski, K. (2004). The development of young children's memory strategies: First findings from the Würzburg Longitudinal Memory Study. Journal of Experimental Child Psychology., 88, 193-209.

Schneider, W., \& Pressley, M. (1997). Memory development between 2 and 20. Mahwah, NJ: Erlbaum.

Schneider, W., Schlagmüller, M., \& Visé, M. (1998). The impact of metamemory and domain-specific knowledge on memory performance. European Journal of Psychology of Education, 13(1), 91-103.

Schneider, W., \& Sodian, B. (1988). Metamemory-memory behavior relationships in young children: Evidence from a memory-for-location-task. Journal of Experimental Child Psychology, 45, 209-233.

Schneider, W., \& Sodian, B. (1991). A longitudinal study of young children's memory behavior and performance in a sort-recall task. Journal of Experimental Child Psychology, 51, 14-29.

Schneider, W., \& Sodian, B. (1997). Memory strategy development: Lessons from longitudinal research. Developmental Review, 17, 442-461.

Schöne, C., Dickhäuser, O., Spinath, B., \& Stiensmeir-Pelster, J. (2003). Das Fähigkeitsselbstkonzept und seine Erfassung. In J. S.-P. F. Rheinberg (Ed.), Diagnostik von Selbstkonzept und Motivation und Selbstregulation. (2 ed., pp. 3-14). Göttingen: Hogrefe.

Schuster-Böck, P. V., \& Zoeke, B. (1991). Zum Erwerb der semantischen Organisationsstrategie - ein Trainingsprogramm für Grundschüler. Paper presented at the 10. Tagung für Entwicklungspsychologie, Köln.

Schutz, P. A., \& Davis, H. A. (2000). Emotions and self-regulation during test taking. Educational Psychologist, 35(4), 243-256.

Schwanenflugel, P. J., Guth, M. E., \& Bjorklund, D. F. (1986). A developmental trend in the understanding of concept attribute importance. Child Development, 57, 421-430.

Scott, M. S., Serchuk, R., \& Mundy, P. (1982). Taxonomic and complementary picture pairs: Abilityin two- to five-years-old. International Journal of Behavioral Development, 5, 243-256.

Scribner, S., \& Cole, M. (1972). Effects of constrained recall training on children's performance in a verbal memory task. Child Development, 43, 845-857.

Shavelson, R. J., Hubner, J. J., \& Stanton, G. C. (1976). Self-concept: Validation of construct interpretations. Review of Educational Research, 46(3), 407-441. 
Shimamura, A. P. (2000). Toward a cognitive neuroscience of metacognition. Consciousness and Cognition, 9(2), 313-323.

Shultz, T. R., Charness, M., \& Berman, S. (1973). Effects of age, social class, and suggestion to cluster on free recall. Developmental Psychology, 8, 57-61.

Siaw, S. N. (1984). Developmental and population comparisons of taxonomic and thematic organization in free recall. Journal of Educational Psychology, 76, 755-765.

Siegler, R. S. (1996). Emerging Minds: The Process of Change in Children's Thinking. Oxford: Universitiy Press.

Siegler, R. S. (2000). The rebirth of children's learning. Child Development, 71(1), 26-35.

Siegler, R. S. (2001a). Children's discoveries and brain-damaged patients' rediscoveries. In J. L. McClelland \& R. S. Siegler (Eds.), Mechanisms of cognitive Development: Behavioral and neural Perspectives. Carnegie Mellon Symposia on Cognition (pp. 3363). Mahwah, N. J.: Lawrence Erlbaum.

Siegler, R. S. (2001b). Das Denken von Kindern. München: R. Oldenbourg.

Siegler, R. S., \& Jenkins, E. (1989). How children discover new strategies. Hillsdale, NJ: Erlbaum.

Siegler, R. S., \& Shrager, J. (1984). Strategy choices in addition and subtraction: How do children know what to do? In C. Sophian (Ed.), Origins of cognitive skills (pp. 229293). Hillsdale, N.J.: Erlbaum.

Skaalvik, E. M., \& Valas, H. (1999). Relations among achievement, self-concept and motivation in mathematics and language arts: A longitudinal study. Journal of Experimental Education, 67(2), 135-149.

Smiley, S. S., \& Brown, A. L. (1979). Conceptual preference for thematic or taxonomic relations: A nonmotoric age trend from preschool to old age. Journal of Experimental Child Psychology, 28, 249-257.

Sodian, B., \& Schneider, W. (1999). Memory strategy development: Gradual increase, sudden insight, or roller coaster? In F. E. Weinert \& W. Schneider (Eds.), Individual Development from 3 to 12: Findings from the Munich Longitudinal Study. Cambridge: Cambridge University Press.

Sodian, B., Schneider, W., \& Perlmutter, M. (1986). Recall, clustering, and metamemory in young children. Journal of Experimental Child Psychology, 41, 395-410.

Sophian, C., \& Wellman, H. M. (1983). Selective information use and perseveration in the search behavior of infants and young children. Journal of Experimental Child Psychology, 35(3), 369-390. 
Stiensmeier-Pelster, J., Balke, S., \& Schlangen, B. (1996). Lern- vs. Leistungszielorientierung als Bedingung des Lernfortschritts. Zeitschrift für Entwicklungspsychologie und Pädagogische Psychologie, 18, 169-187.

Stipek, D. (1984). Young children`s performance expectations: Logical analysis or wishful thinking? In J. G. Nicholls (Ed.), Advances in motivation and achievment (Vol. 3, pp. 33-56). Greenwich, CT: JAI Press.

Stipek, D., \& MacIver, D. (1989). Developmental change in children's assessment of intellectual competence. Child Development, 60(3), 521-538.

Tewes, U., Schallberger, U., \& Rossmann, K. (2000). Hamburg-Wechsler-Intelligenztest für Kinder III (HAWIK III). Göttingen: Hogrefe.

Tulving, E. (1962). Subjective organizationin free recall of "unrelated words". Psychological Review, 69, 344-345.

Tulving, E. (1972). Episodic and semantic memory. In E. Tulving \& W. Donaldson (Eds.), Organization of memory (pp. 381-403). New York: Academic Press.

Tulving, E. (1982). Synergistic ecphory in recall and recognition. Canadian Journal of Psychology., 36, 130-147.

Tulving, E. (2000). Concepts of memory. In E. Tulving \& F. J. M. Craik (Eds.), The Oxford Handbook of Memory (pp. 33-43). Oxford: University Press.

Tulving, E., \& Madigan, S. (1970). Memory and verbal Learning. Annual Review of Psychology, 21, 437-484.

Turner, J. E., Henry, L. A., \& Smith, P. T. (2000). The development of the use of long-term knowledge to assist short-term recall. The Quarterly Journal of Experimental Psychology A, 53(2), 457-478.

van Kraayenoord, C. E., \& Schneider, W. E. (1999). Reading achievement, metacognition, reading self-concept and interest: A study of German students in grades 3 and 4 . European Journal of Psychology of Education, 14(3), 305-324.

von Aken, M. A. G., Helmke, A., \& Schneider, W. (1997). Selbstkonzept und Leistung Dynamik ihres Zusammenspiels: Ergebnisse aus dem SCHOLASTIK-Projekt. In F. E. Weinert \& A. Helmke (Eds.), Entwicklung im Grundschulalter (pp. 341-350). Weinheim: Beltz, Psychologie Verlags Union.

Waters, H. S. (1982). Memory development in adolescence: Relationships between metamemory, strategy use, and performance. Journal of Experimental Child Psychology, 33, 183-195. 
Waters, H. S. (2000). Memory strategy development: Do we need yet another deficiency? Child Development, 71(4), 1004-1012.

Waters, H. S., \& McAlaster, R. (1983). Encoding variability and organization in free recall. Journal of Experimental Child Psychology, 36, 380-395.

Waters, H. S., \& Schreiber, L. L. (1991). Sex differences in elaborative strategies: A developmental analysis. Journal of Experimental Child Psychology, 52(3), 319-335.

Waters, H. S., \& Waters, E. (1976). Semantic processing in children's free recall: Evidence for the importance of attentional factors and encoding variability. Journal of Experimental Psychology: Human Learning and Memory, 2, 370-380.

Waters, H. S., \& Waters, E. (1979). Semantic processing in children's free recall: The effects of context and meaningsfulness on encoding variability. Child Development, 45, 735746.

Weber, A., \& Stefanek, J. (1998). Überblick über die Längsschnittstudie LOGIK. In F. E. Weinert (Ed.), Entwicklung im Kindesalter. Weinheim: Beltz, Psychologie Verlags Union.

Weinert, F. E. (1984). Metakognition und Motivation als Determinanten der Lerneffektivität: Einführung und Überblick. In F. E. W. R. H. Kluwe (Ed.), Metakognition, Motivation und Lernen (pp. 9-21). Stuttgart:: Kohlhammer.

Weissberg, J. A., \& Paris, S., G. (1986). Young children's remembering in different contexts: A reinterpretation of Istomina's study. Child Development, 57(5), 1123-1129.

Wellman, H. M. (1977a). The early development of intentional memory behavior. Human Development, 20, 86-101.

Wellman, H. M. (1977b). Preschooler's understanding of memory-relevant variables. Child Development, 48, 1720-1723.

Wellman, H. M. (1983). Metamemory revisited. In M. T. H. Chi (Ed.), Trends in memory development research. (pp. 31-51). Basel: Karger.

Wellman, H. M. (1985b). The origins of metacognition. In G. E. M. T. G. W. D. L. ForrestPressley (Ed.), Metacognition, cognition, and human performance. (Vol. 1, pp. 1-31). Orlando, Florida: Academic Press.

Wilkinson, A. C., De Marinis, M., \& Riley, S. J. (1983). Developmental and individual differences in rapid remembering. Child Development, 54, 898-911.

Wilkinson, A. C., \& Koestler, R. (1983). Repeated Recall: A new model and tests of its generality from childhood to old age. Journal of Experimental Psychology, 112, 423451. 
Wilkinson, A. C., \& Koestler, R. (1984). Generality of Markov model for repeated recall. Journal of Mathematical Psychology, 28, 43-72.

Williams, K. G., \& Goulet, L. R. (1975). The effects of cueing and constraint instructions on children's free recall performance. Journal of Experimental Child Psychology, 19, 464-475.

Wimmer, H., \& Tornquist, K. (1980). The role of metamemory and metamemory activation in the development of mnemonic performance. International Journal of Behavioral Development, 3, 71-81.

Woody-Dorning, J., \& Miller, D. J. (2001). Children's individual differences in capacity: Effects on strategy production and utilization. British Journal of Developmental Psychology, 19(4), 543-557.

Woody-Ramsey, J., \& Miller, P. H. (1988). The facilitation of selective attention in preschoolers. Child Development, 59, 1497-1503.

Worden, P. E. (1974). The development of the category-recall function under three retrieval conditions. Child Development, 45, 1054-1059.

Worden, P. E. (1976). The effects of clasification structure on organized free recall in children. Journal of Experimental Child Psychology, 22, 519-529.

Zanobini, M., \& Usai, C. (2002). Domain-specific self-concept and achievement motivation in the transition from primary to low middle school. Educational Psychology, 22(2), 203-217. 


\section{Anhang A}

\section{Untersuchungsmaterial: Sort Recall-Aufgabe}

Eingesetztes Itemmaterial (Liste 1)

\begin{tabular}{|l|l|l|l|l|}
\hline Kategorie & \multicolumn{4}{|c|}{ Items } \\
\hline Büroartikel & Bleistift & Lineal & Schere & Spitzer \\
\hline Medizinartikel & Fieberthermometer & Pflaster & Spritze & Tabletten \\
\hline Lebensmittel & Eis & Hamburger & Kuchen & Schinken \\
\hline Behältnisse & Koffer & Korb & Rucksack & Tasche \\
\hline Haushaltsgeräte & Bügeleisen & Kaffeemaschine & Toaster & Waschmaschine \\
\hline
\end{tabular}

Eingesetztes Itemmaterial (Liste 2) in alphabetischer Reihenfolge

\begin{tabular}{|c|c|c|c|c|}
\hline & \multicolumn{4}{|c|}{ Messzeitpunkt } \\
\hline & 1. & 2. & 3. - 4 . & 5. \\
\hline Werkzeuge & $\begin{array}{c}\text { Hammer } \\
\text { Säge } \\
\text { Schraubenzieher } \\
\text { Zange }\end{array}$ & $\begin{array}{l}\text { Hammer } \\
\text { Nagel } \\
\text { Säge } \\
\text { Zange }\end{array}$ & $\begin{array}{c}\text { Bohrmaschine } \\
\text { Axt } \\
\text { Hobel } \\
\text { Schaufel } \\
\text { Meissel } \\
\end{array}$ & $\begin{array}{c}\text { Bohrmaschine } \\
\text { Axt } \\
\text { Hobel } \\
\text { Schaufel } \\
\text { Meissel } \\
\end{array}$ \\
\hline Früchte & $\begin{array}{l}\text { Banane } \\
\text { Birne } \\
\text { Orange } \\
\text { Pflaume }\end{array}$ & $\begin{array}{l}\text { Ananas } \\
\text { Apfel } \\
\text { Birne } \\
\text { Erdbeere }\end{array}$ & $\begin{array}{l}\text { Weintraube } \\
\text { Melone } \\
\text { Nuss } \\
\text { Ananas } \\
\text { Pfirsich } \\
\end{array}$ & $\begin{array}{c}\text { Weintraube } \\
\text { Melone } \\
\text { Nuss (Haselnuss) } \\
\text { Ananas } \\
\text { Pfirsich } \\
\end{array}$ \\
\hline Möbel & $\begin{array}{l}\text { Bett } \\
\text { Sessel } \\
\text { Stuhl } \\
\text { Tisch }\end{array}$ & $\begin{array}{l}\text { Regal } \\
\text { Schrank } \\
\text { Sessel } \\
\text { Stuhl }\end{array}$ & $\begin{array}{l}\text { Schaukelstuhl } \\
\text { Kommode } \\
\text { Spiegel } \\
\text { Hocker } \\
\text { Schreibtisch }\end{array}$ & $\begin{array}{l}\text { Schaukelstuhl } \\
\text { Kommode } \\
\text { Spiegel } \\
\text { Hocker } \\
\text { Schreibtisch } \\
\end{array}$ \\
\hline Kleidung & $\begin{array}{c}\text { Hose } \\
\text { Pullover } \\
\text { Schuhe } \\
\text { Unterhose }\end{array}$ & $\begin{array}{c}\text { Hose } \\
\text { Jacke } \\
\text { Strümpfe } \\
\text { T-Shirt }\end{array}$ & $\begin{array}{c}\text { Bluse } \\
\text { Badeanzug } \\
\text { Krawatte } \\
\text { Stiefel } \\
\text { Unterhemd }\end{array}$ & $\begin{array}{c}\text { Bluse } \\
\text { Badeanzug } \\
\text { Krawatte } \\
\text { Stiefel } \\
\text { Unterhemd }\end{array}$ \\
\hline Körperteile & $\begin{array}{l}\text { Auge } \\
\text { Hand } \\
\text { Nase } \\
\text { Ohr }\end{array}$ & $\begin{array}{l}\text { Bauch } \\
\text { Hand } \\
\text { Nase } \\
\text { Zähne }\end{array}$ & $\begin{array}{c}\text { Herz } \\
\text { Gehirn } \\
\text { Zeh } \\
\text { Fingernagel } \\
\text { Zähne } \\
\end{array}$ & $\begin{array}{c}\text { Herz } \\
\text { Gehirn } \\
\text { Zeh } \\
\text { Fingernagel } \\
\text { Zähne } \\
\end{array}$ \\
\hline Fahrzeuge & - & - & - & $\begin{array}{c}\text { Kran } \\
\text { Hubschrauber } \\
\text { Traktor } \\
\text { Dreirad } \\
\text { Wohnwagen }\end{array}$ \\
\hline
\end{tabular}




\section{Anhang B}

Kategoriale Typizität der Items

\begin{tabular}{|c|c|c|c|c|c|c|}
\hline & \multicolumn{2}{|l|}{ Klasse 2} & \multicolumn{2}{|l|}{ Klasse 4} & \multirow{2}{*}{$\begin{array}{l}\text { Klasse } 6 \\
\text { neu }\end{array}$} \\
\hline & & Hasselhorn & neu & Hasselhorn & neu & \\
\hline \multirow{5}{*}{ Liste 1} & Büroartikel & \multirow{5}{*}{$\begin{array}{l}\text { nicht } \\
\text { erhoben }\end{array}$} & .06 & \multirow{5}{*}{$\begin{array}{l}\text { nicht } \\
\text { erhoben }\end{array}$} & .04 & .14 \\
\hline & Medizinartikel & & .17 & & .28 & .33 \\
\hline & Lebensmittel & & .04 & & .06 & .05 \\
\hline & Behältnisse & & .04 & & .05 & .13 \\
\hline & Haushaltsgeräte & & .02 & & .13 & .13 \\
\hline \multirow{5}{*}{ Liste 2} & Werkzeuge & .64 & .49 & .64 & .54 & .61 \\
\hline & Früchte & .54 & .37 & .53 & .47 & .47 \\
\hline & Möbel & .60 & .51 & .60 & .50 & .55 \\
\hline & Kleidung & .70 & .50 & .71 & .56 & .51 \\
\hline & Körperteile & .58 & .35 & .60 & .54 & .46 \\
\hline \multirow{6}{*}{ Liste 2} & Werkzeuge & .06 & .07 & .16 & .11 & .13 \\
\hline & Früchte & .09 & .10 & .20 & .18 & .22 \\
\hline & Möbel & .08 & .04 & .15 & .14 & .14 \\
\hline & Kleidung & .14 & .12 & .23 & .21 & .27 \\
\hline & Körperteile & .13 & .07 & .25 & .12 & .20 \\
\hline & Fahrzeuge & .24 & .05 & .16 & .06 & .07 \\
\hline
\end{tabular}

Mittlere kategoriale Typizität der Items in Abhängigkeit von der Liste in den jeweiligen Messzeitpunkten (Standardabweichung in Klammern)

\begin{tabular}{|l|l|l|l|l|l|}
\hline \multicolumn{2}{|l|}{} & $\begin{array}{l}\text { T 1-2 } \\
\text { Ende Klasse 2 }- \\
\text { Anfang Klasse 3 }\end{array}$ & $\begin{array}{l}\text { T 3- } \\
\text { Ende Klasse 3 }- \\
\text { Ende Klasse 4 }\end{array}$ & $\begin{array}{l}\text { T- 4 } \\
\text { Ende Klasse 3 - } \\
\text { Ende Klasse 4 (ink1. } \\
\text { Fahrzeuge) }\end{array}$ \\
\hline Liste 1 & Bei 3 Klassenstufen & \multicolumn{2}{|c|}{$.11(.05)$} \\
\hline
\end{tabular}




\section{Anhang C}

\section{Item I (Metagedächtnisfragebogen)}

Hier siehst du drei Listen mit Bildern. Wenn du versuchen müsstest, die Bilder der Liste auswendig zu lernen, welche Liste glaubst du, ist einfacher zu lernen? Sieh dir die erste Liste an und schau auf die Bilder. Du hast jetzt einige Sekunden Zeit, um die drei Listen sorgfältig durchzusehen. Du sollst dich dann entscheiden, welche Liste am leichtesten und welche am schwersten zu lernen ist, wenn du die Bilder auswendig nennen solltest. Du darfst anschließend Noten vergeben. Einer leicht zu lernenden Liste gibst du eine gute Note, einer mittelguten eine mittelgute Note, und einer schweren Liste gibst du eine schlechte Note. Schreibe die Noten bitte in die Kästchen davor. Du kannst eine Note auch mehrmals vergeben.

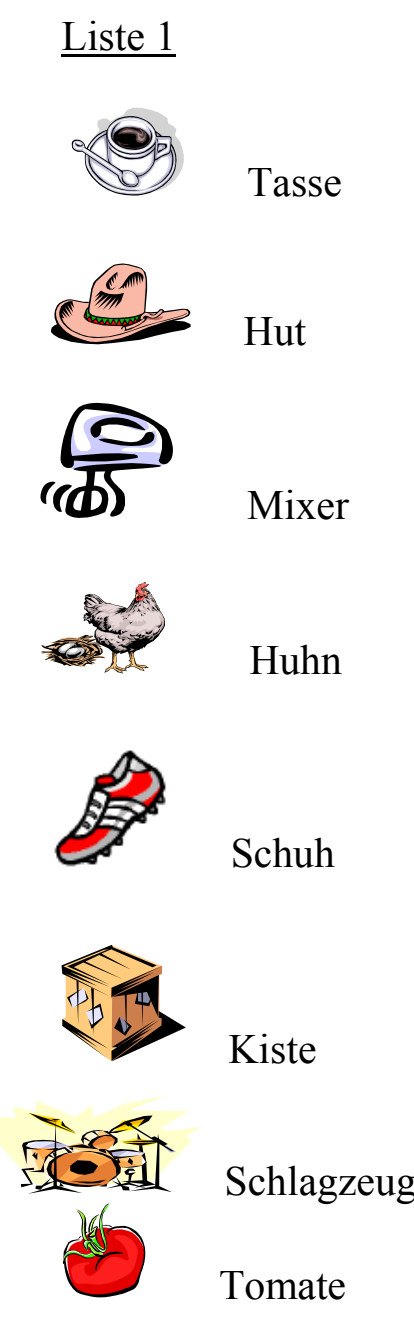

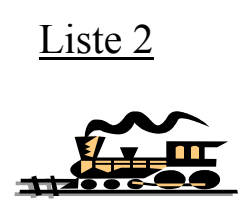
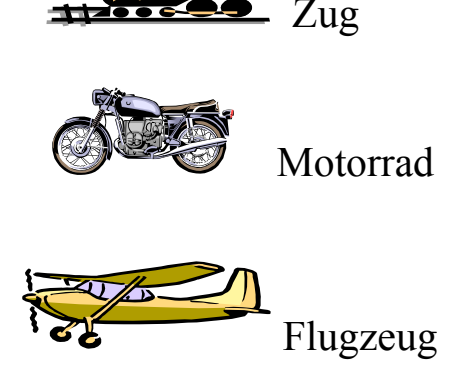

Orange

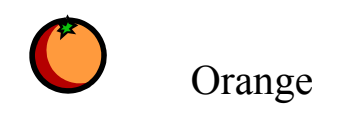

Banane
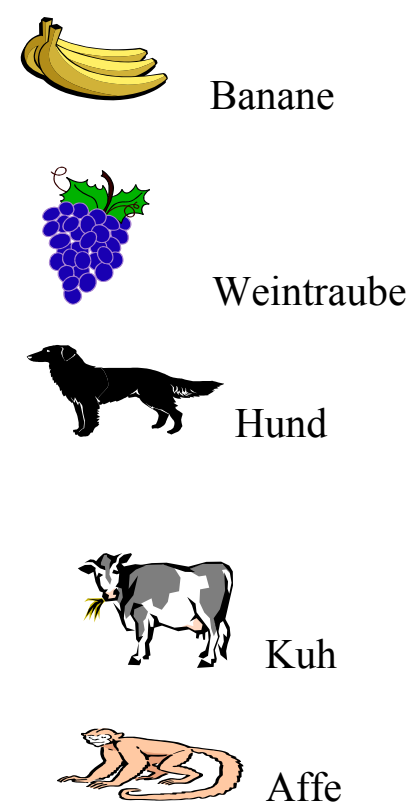

\section{$\underline{\text { Liste } 3}$}
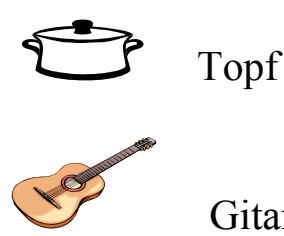

Gitarre

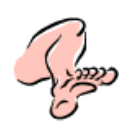

Bein
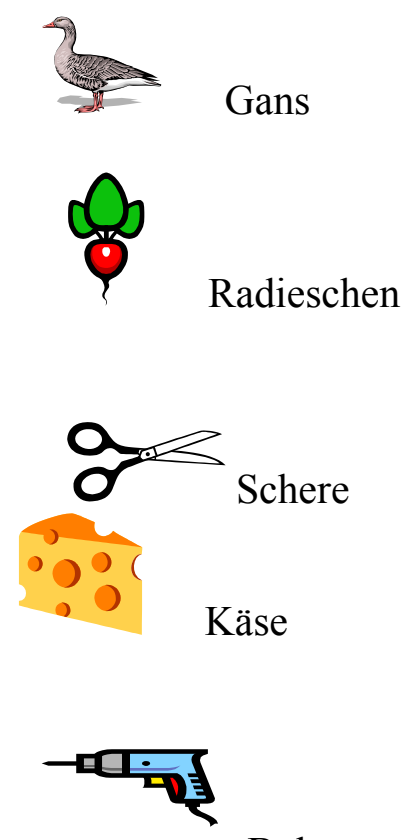

Bohrer

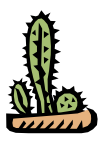

Kaktus 


\section{Anhang D}

Item II (Metagedächtnisfragebogen)

Hier unten siehst du drei Listen mit Wörtern. Du darfst nun Noten dafür geben, wie leicht man die Listen lernen kann (1-5). Einem guten Vorschlag gibst du eine gute Note, einem mittelguten eine mittelgute Note, und einem schlechten Vorschlag gibst du eine schlechte Note.

\begin{tabular}{|c|c|}
\hline Liste 1 & $\begin{array}{l}\text { Gabel, Buch, Fußball, } \\
\text { Auto, Hand, Sonne, See, } \\
\text { Wand, Baum }\end{array}$ \\
\hline Liste 2 & $\begin{array}{c}\text { Trommel, Hose, Hut, } \\
\text { - - } \\
\text { Tisch, Schuh, Flöte, } \\
\text { - - - } \\
\text { Schrank, Geige, Sofa }\end{array}$ \\
\hline Liste 3 & $\begin{array}{c}\text { Hammer, Zange, Säge, } \\
\text { - - } \\
\text { Apfel, Kirsche, Birne, } \\
\text { - - - } \\
\text { Katze, Pferd, Huhn }\end{array}$ \\
\hline
\end{tabular}

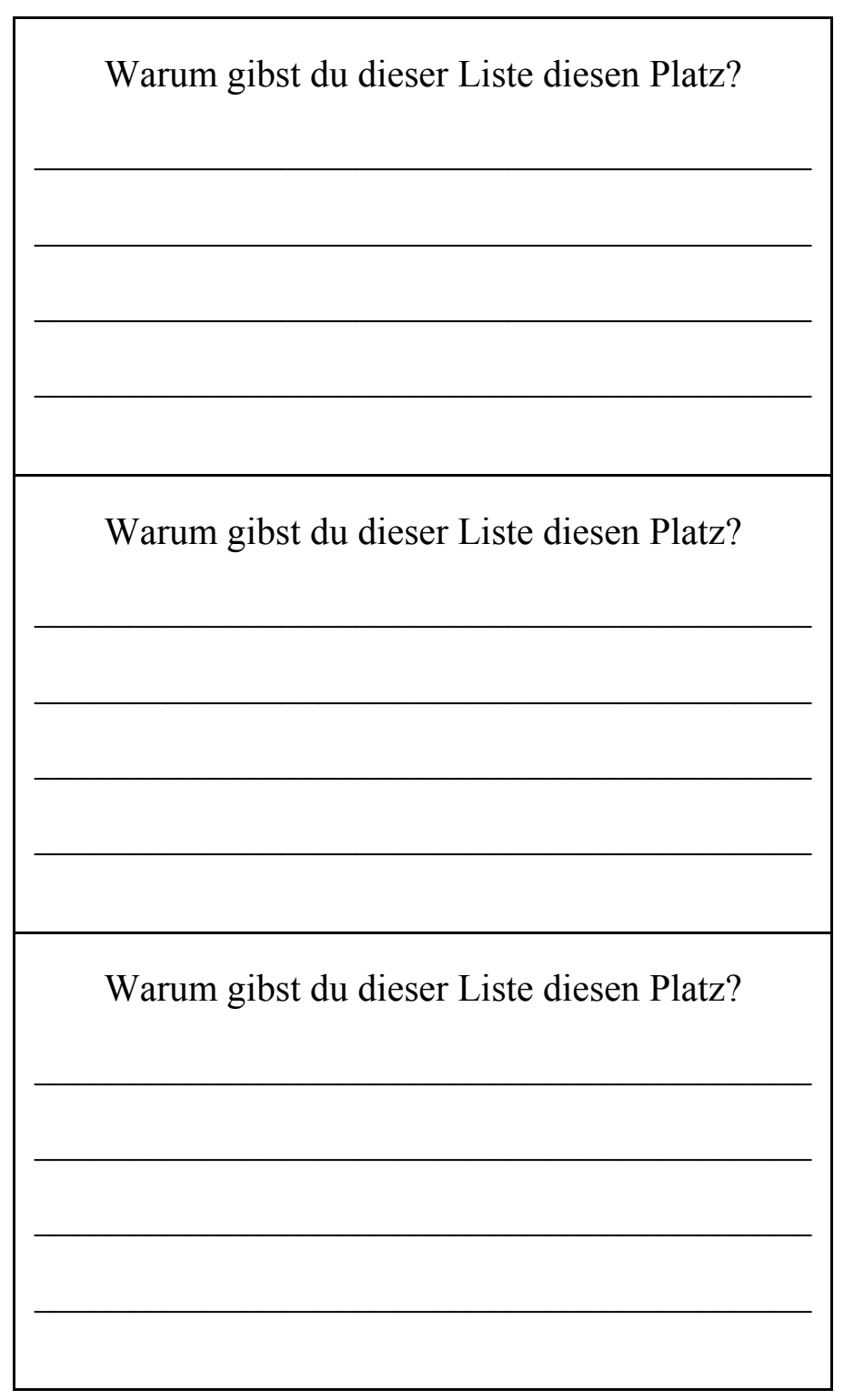

Schreibe die Noten bitte vorne in die Kästchen. Du kannst eine Note auch mehrmals vergeben. 


\section{Anhang E}

\section{Untersuchungsmaterial: Zahlennachsprechen vorwärts}

\begin{tabular}{l|l} 
Gruppe: & \multicolumn{2}{l}{ Bemerkungen: } \\
1) & $\begin{array}{l}2-9 \\
4-6\end{array}$ \\
\hline 2) & $\begin{array}{l}3-8-6 \\
6-1-2\end{array}$ \\
\hline 3) & $\begin{array}{l}3-4-1-0 \\
6-1-5-8\end{array}$ \\
\hline 4) & $\begin{array}{l}8-4-2-3-9 \\
5-2-1-8-6\end{array}$ \\
\hline 5) & $\begin{array}{l}3-8-9-1-0-4 \\
0-9-6-4-8-3\end{array}$ \\
\hline 6) & $5-1-0-4-2-3-8$ \\
& $9-8-5-2-1-6-3$ \\
\hline 7) & $1-6-4-5-9-0-6-3$ \\
& $2-9-0-6-3-1-5-4$ \\
\hline 8$)$ & $\begin{array}{l}5-3-8-0-1-2-4-6-9 \\
4-2-6-9-1-0-8-3-5\end{array}$ \\
\hline
\end{tabular}

Untersuchungsmaterial: Zahlennachsprechen rückwärts

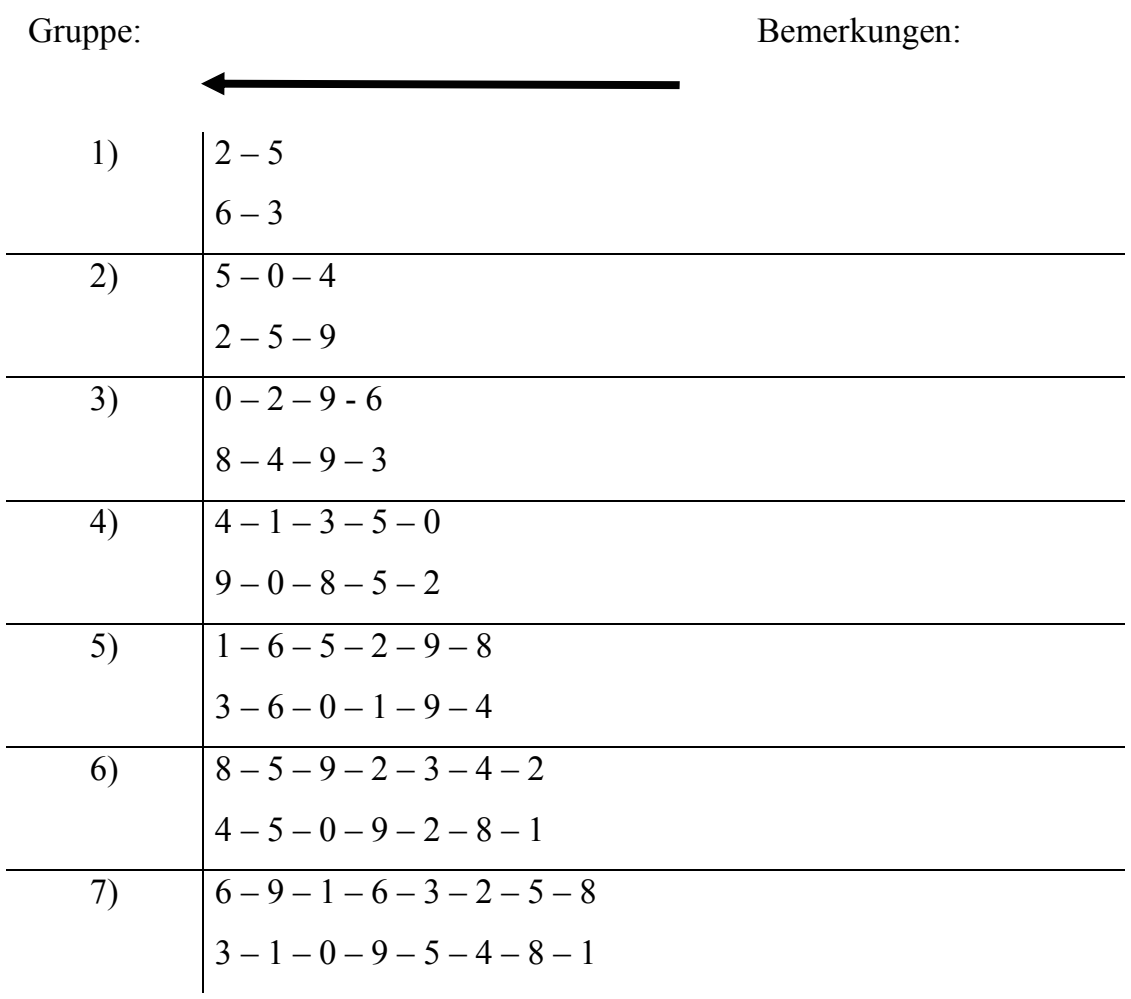




\section{Anhang F}

\section{Lernmaterial: Motivationsfragebogen}

1. Wie viel Spaß machen dir solche Spiele, bei denen du dir BILDER MERKEN musst?

2. Wie viel Spaß macht es dir, dir ZAHLEN zu merken?

3. Wie viel Spaß macht es dir, dir WÖRTER zu merken?

4. Wie viel Spaß macht es dir, GEHEIMWÖRTER nachzusprechen?

5. Wie viel Spaß macht es dir, GEDICHTE auswendig zu lernen?

6. Wie viel Spaß macht es dir, dir GESCHICHTEN zu merken?

7. Wie viel Spaß machen dir Merkspiele, wie z.B. MEMORY?

8. Wie viel Spaß macht es dir, etwas für MATHEMATIK zu lernen?

9. Wie viel Spaß macht es dir, etwas für DEUTSCH zu lernen?

10. Wie viel Spaß macht es dir, etwas für HEIMAT- UND SACHKUNDE zu lernen?

11. Wie viel Spaß macht es dir, Lieder zu lernen (z.B. im MUSIKUNTERRICHT)?

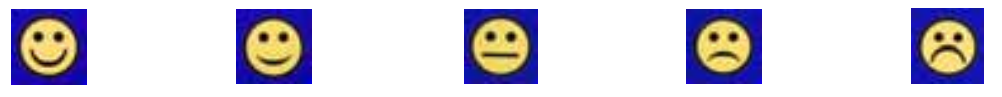

sehr viel Spaß viel Spaß etwas $\mathrm{Spa} \quad$ nicht viel $\mathrm{Spa} ß$ überhaupt keinen $\mathrm{Spa} ß$ 
Anhang G:

\section{Köpfchenliste}

Der Reste

„Schau dir nun mal diese Seite an, da siehst du lauter kleine Köpfchen.

Diese Köpfchen sollen alle Kinder aus deiner Klasse sein.

Wir tun jetzt mal so, als würden sie alle so aussehen.

A In dieser Spalte sind nun alle Kinder deiner Klasse danach sortiert, wie gut sie im DIKTAT oder in der NACHSCHRIFT sind.

(Weißt du, was ein Diktat oder eine Nachschrift ist? Wenn nein:

Hierbei geht es darum, wie gut du Wörter richtig schreiben kannst.)

Der Allerbeste im Diktat (Nachschrift) ist ganz oben in der Reihe.

Der macht nie einen Fehler. Der Schlechteste im Diktat steht ganz unten.

Wörter richtig schreiben liegt dem überhaupt nicht. Das Kind, das mittelgut im Diktat ist, ist in der Mitte von der Reihe - da wo der Strich ist. Mach jetzt ein Kreuz dahin, wo du in dieser Reihe stehst, wenn die Kinder deiner Klasse danach sortiert sind, wie gut sie im Diktat (Nachschrift) sind.

B ... MATHEMATIK ...

C ... SPORT...

D ... BILDER MERKEN ...

E ... ZAHLEN MERKEN ...

F ... WÖRTER MERKEN ...

G ... WIEDERFINDEN, DAS VERSTECKT ...

H ... GESCHICHTEN NACHERZÄHLEN ... 


\section{Anhang H:}

\section{Lernmaterial: Wortschatztest}

1. Was ist ein Brot?

2. Was ist ein Regenschirm?

3. Was ist ein Esel?

4. Was ist ein Hut?

5. Was bedeutet Teilnehmen?

6. Was ist das Alphabet?

7. Was bedeutet Mutig?

8. Was ist eine Sekunde?

9. Was ist eine Insel?

10. Was bedeutet Unsinn?

11. Was bedeutet Auswandern?

12. Was bedeutet Prahlen?

13. Was bedeutet Scheitern?

14. Was bedeutet Anstrengend?

15. Was ist ein Verhör?

16. Was bedeutet Durchsichtig?

17. Was ist eine Fabel?

18. Was ist ein Leiden?

19. Was bedeutet Präzise?

20. Was ist ein Konflikt?

21. Was bedeutet Konkurrenz?

22. Was bedeutet Bevorstehend?

23. Was bedeutet Erzwingen?

24. Was ist eine Abänderung?

25. Was ist ein Inserat?

26. Was bedeutet Abgeschiedenheit?

27. Was bedeutet Labil?

28. Was bedeutet Abstrakt?

29. Was ist eine Anomalie

30. Was ist eine Hierarchie? 


\section{Anhang I:}

\section{Lernmaterial: Allgemeines Wissen}

1) Wie heißt das hier? (Testleiterin zeigt auf ihre Nase)

2) Wie viele Beine hat ein Hund?

3) Was musst du machen, damit das Wasser kocht?

4) Welcher Tag kommt nach dem Donnerstag?

5) Sag mir den Namen von zwei Geldstücken!

6) Wie viele Tage hat die Woche?

7) Welcher Monat kommt nach dem März?

8) Wie heißen die vier Jahreszeiten?

9) Wie viele Stunden hat der Tag?

10) Was macht der Magen?

11) Wer war Christoph Kolumbus?

12) Welcher Monat ist alle vier Jahre einen Tag länger als sonst?

13) Wie wird der Sauerstoff in der Luft erneuert?

14) Wie viel Stück hat ein Dutzend?

15) In welcher Himmelsrichtung geht die Sonne unter?

16) Auf welchem Kontinent liegt Brasilien?

17) Was sind Hieroglyphen?

18) Wie heißt die Hauptstadt von Griechenland?

19) Wer war Anne Frank?

20) Welches Land der Welt hat die meisten Einwohner?

21) Aus welchem Grund rostet Eisen?

22) Welches Metall ist bei normaler Temperatur flüssig?

23) Warum haben Pflanzen meistens grüne Blätter?

24) Woraus besteht Wasser?

25) Was ist ein Barometer?

26) Wie weit ist es von Berlin nach München?

27) Warum donnert es bei Gewitter?

28) Welches ist das wichtigste Material, das man braucht, um Glas herzustellen?

29) Wie groß ist der Erdumfang?

30) Wer war Charles Darwin? 


\section{Lebenslauf}

Persönliche Angaben

\section{Schulausbildung}

1986-1992

\section{Hochschulausbildung}

Apr. 1993 - Sept. 1996

Okt. 1996 - Sept. 2000
Maren Richter, geb. Guternacht

Bierweg 3

37120 Bovenden - Eddigehausen

geb. am 11 April 1973 in Bad Pyrmont

verheiratet

Humboldt-Gymnasium Bad Pyrmont

Abitur im Mai 1992 (Abschlussnote: 1,8)

Medizin-Studium an der Georg-August-Universität in Göttingen

Psychologie-Studium Georg-August-Universität, Göttingen

Diplom im September 2000 (Abschlussnote: 1,3)

\section{Berufspraktika:}

Febr. - Apr. 1999

Juli - Aug. 1999

\section{Berufstätigkeit:}

seit Okt. 2000

Wissenschaftl. Mitarbeiterin der Abeilung Pädagogische

Psychologie und Entwicklungspsychologie des Georg-Elias-

Müller Instituts der Universität Göttingen

Bearbeitung des DFG-Projektes „Bedingungen und

intraindividuelle Entwicklungsverläufe strategischer

Gedächtnisprozesse zwischen 5 und 12 Jahren“ 